\title{
Understanding the Dynamics of Online Social Conformity
}

Senuri Wijenayake

ORCID: 0000-0003-1985-0227

Doctor of Philosophy

September, 2021

School of Computing and Information Systems

Faculty of Engineering and Information Technology

The University of Melbourne, Australia

Submitted in total fulfilment of the requirements for the degree of Doctor of Philosophy. 



\section{Abstract}

Social conformity occurs when people adjust their personal opinions and behaviours to agree with contradictory perspectives of group majorities. While conformity has been established as a powerful social influence in face-to-face groups, its impact in online groups remains unclear. Informed by a thorough literature review of conformity research, this thesis systematically investigates the effects of several popular contextual and personal determinants of conformity behaviour (as reported by prior work in physical groups), to quantify their effects in different online group settings. In brief, our findings show that people are more receptive to conformity influences when challenged by larger than smaller majorities, in tasks with a clear "correct" answer. We also note that an individual's susceptibility to conformity increases in online group settings with higher social presence. In addition, personal aspects such as age and gender related stereotypical perceptions of self and peer competency, self-confidence on personal judgement, and certain personality traits also determine users' susceptibility to conformity influences in online groups.

The thesis also expands on potential outcomes of online social conformity behaviour, to reveal both positive (e.g., encouraging prosocial behaviours in the dissemination of online news articles) and negative (e.g., accepting incorrect responses of a group's majority as accurate) outcomes. Hence, we describe social conformity as a powerful social phenomenon that has the potential to be used to encourage positive interaction in online group settings - given that we also account for its capacity for adverse outcomes through appropriate platform design. As such, we provide several design guidelines that demonstrate how certain conformity determinants (e.g., social presence) can be embedded in online platform design to effectively control perceived social conformity influences in online group settings. We further encourage future work to build on our findings on this regard to evaluate the effectiveness of the proposed guidelines in practice.

We conclude with a discussion on possible next steps in online conformity research. Concisely, we motivate the need for future research to examine the effects of social conformity in more realistic online environments, where social pressure situations occur naturally. Moreover, while this thesis examined effects of several popular contextual and personal determinants on online social conformity, this did not constitute a complete list of potential determinants. This is especially crucial given that the focus of conformity research is now shifting from physical to online settings, and as a result certain determinants that may have not been of significance in physical groups, will become increasingly relevant. We also emphasise that there is a lack of research that examines online conformity behaviour in terms of its longevity. Doing so is vital to distinguish between its short-term and long-term effects, in order to truly understand how social conformity influences manifest and sustain in our everyday online social interactions. 



\section{Declaration}

This is to certify that:

1. the thesis comprises only my original work towards the $\mathrm{PhD}$,

2. due acknowledgement has been made in the text to all other material used,

3. appropriate ethics procedure and guidelines have been followed to conduct this research,

4. the thesis is less than 100,000 words in length, exclusive of tables, maps, bibliographies and appendices.

Senuri Wijenayake

September 2021 



\section{Preface}

This thesis is submitted in total fulfilment of the requirements for the degree of Doctor of Philosophy at the University of Melbourne. The entirety of the work presented here was conducted during my PhD candidature at the School of Computing and Information Systems, The University of Melbourne, under the supervision of Dr. Jorge Goncalves and Prof. Vassilis Kostakos.

The thesis includes five peer-reviewed articles, the details of which are provided below. Furthermore, while several co-authors have contributed to these articles, I declare that I am the primary author and have contributed for more than $50 \%$ of the work in each article. More specifically, I proposed the research questions, planned the study design, obtained the necessary ethical clearance from the The University of Melbourne Human Ethics Advisory Group (application IDs given below), developed the software for data collection and analysis, recruited participants, and collected user data. Additionally, I drafted the research articles for submission and subsequently revised them based on the peer-review feedback received. Moreover, I am grateful for the contributions of the listed co-authors, who provided feedback to refine the proposed study designs, suggested data analysis techniques, contributed to the preparation of the research articles, and helped address revisions. Hence, I use the scientific term "we" throughout the main chapters of this thesis in recognition of my co-authors' contributions.

The research articles included in this thesis are referred by roman numerals (Article IV) as indicated below. All articles are included in full (author-approved version), preceded by a succinct introduction situating each article within the context of the thesis.

\section{Article I}

Wijenayake, S., van Berkel, N., Kostakos, V., Goncalves, J. (2020). Impact of Contextual and Personal Determinants on Online Social Conformity. Computers in Human Behavior, 108, 106302. https://doi.org/10.1016/j.chb.2020.106302.

Ethics Application ID: 1853038

Published by Elsevier on February 2020.

\section{Article II}

Wijenayake S., Hu J., Kostakos V., Goncalves J. (2021). Quantifying the Effects of AgeRelated Stereotypes on Online Social Conformity. In: Ardito C. et al. (eds) HumanComputer Interaction - INTERACT 2021. INTERACT 2021. Lecture Notes in Computer Science, vol 12935. Springer, Cham. https://doi.org/10.1007/978-3-030-85610-6_26.

Ethics Application ID: 2056161 
Published by Springer, Cham on August 2021.

\section{Article III}

Wijenayake, S., van Berkel, N., Kostakos, V., Goncalves, J. (2019). Measuring the Effects of Gender on Online Social Conformity. Proceedings of the ACM on Human-Computer Interaction, 3(CSCW), 1-24. https://doi.org/10.1145/3359247.

Ethics Application ID: 1853038

Published by ACM on November 2019.

\section{Article IV}

Wijenayake, S., Van Berkel, N., Kostakos, V., Goncalves, J. (2020). Quantifying the Effect of Social Presence on Online Social Conformity. Proceedings of the ACM on HumanComputer Interaction, 4(CSCW1), 1-22. https://doi.org/10.1145/3392863.

Ethics Application ID: 1954591

Published by ACM on May 2020.

\section{Article V}

Wijenayake, S., Hettiachchi, D., Hosio, S., Kostakos, V., Goncalves, J. (2020). Effect of Conformity on Perceived Trustworthiness of News in Social Media. IEEE Internet Computing, 25(1), 12-19. https://doi.org/10.1109/MIC.2020.3032410.

Ethics Application ID: 2056168

Published by IEEE on October 2020

I gratefully acknowledge the following sources of funding that supported this research:

- Melbourne Research Scholarship

- Rowden White Scholarship

- Diane Lemaire Scholarship

- Faculty of Engineering and Information Technology Conference Travel Scholarship

- School of Computing and Information Systems Conference Travel Scholarship

Articles I, II and V received minimal editorial editing in the regular publication process. No other third-party editorial assistance was provided in preparation of the thesis. The studies presented in Article I-V received ethical clearance from The University of Melbourne Human Ethics Advisory Group under the indicated ethics applications. 


\section{Acknowledgements}

To start with, I am very grateful for the guidance, support and company of my supervisors Dr. Jorge Goncalves and Prof. Vassilis Kostakos. Thank you for being excited and invested in my $\mathrm{PhD}$ - as much as I have been. I could not have asked for a better combination of supervisors. I wholeheartedly thank Jorge, for believing in me, taking care of my mental and social well-being, and for encouraging me to take up every opportunity that I came across. You did $100 \%$ and above, to make sure I succeed in this journey. I really appreciate that you took the time to understand what I wanted to do in life post-PhD, to best prepare me for the challenges ahead. I will always look back to the conversations we had and try my best to make you proud going forward. Many thanks to Vas, who introduced me to new perspectives of research and life. Thank you for being so helpful, whenever I needed input on my research plans, as well as my academic career. You are an exemplary leader! Special thanks to Assoc. Prof. Sherah Kurnia from CIS, for going above and beyond the role of an Advisory Chair, and being a mentor and a friend to me. Thank you so much, for your continuous support throughout my $\mathrm{PhD}$. There are no words to describe how immensely grateful I am for having the privilege of your company and wisdom.

Next, I must mention Dr. Niels van Berkel and Dr. Danula Hettiachchi who helped me with my research proposal and $\mathrm{PhD}$ application, that finally culminated in a $\mathrm{PhD}$ at University of Melbourne. Also, thank you for the very warm welcome, when I joined the $\mathrm{HCI}$ lab in 2018. As a fresh $\mathrm{PhD}$ student, I was trilled that you made me a part of your research, which played a significant role in my transition into the $\mathrm{PhD}$ life. Additionally, kudos to my mentors and friends Dr. Zhanna Sarsenbayeva, Dr. Joshua Newn, Dr. Chu Luo and Gabriele Marini for being stellar role models. And to my closest friends Dr. Unni Krishnan and Dr. Namrata Srivastava - you are amazing! You have played a big role in my life, and I will continue to look up to you. Thank you for making me smile and have fun through my $\mathrm{PhD}$. Your friendship has been the best gift I could ask for!

I am very grateful for the support and company of everyone at the HCI group. Special thanks to Dr. Eduardo Velloso, Dr. Tilman Dingler, Dr. Benjamin Tag and Dr. Sarah Webber for taking the time to refine my research plans. Your input added significant value to the work presented in this thesis. I am also humbled to have worked with Dr. Melissa Rogerson, who not only kick started my career as a tutor at the university, but also continue to inspire me to be a better teacher! I would also take this opportunity to thank Dr. Nicole Barbee, Rhonda Smithies, Zaher Joukhadar, and Allen Pilares for the amazing work they do at CIS, to make everyone's life so much easier! You have been an immense strength to me from my very first day. You are superstars!

The HCI lab has an amazing set of people that I have had the privilege of getting know at different capacities including Ebrahim Babaei, Henrietta Lyons, Sara Khorsani, Martin Reinoso, Brandon Syiem, Priyankar Bhattacharjee, Qiushi Zhou, Difeng Yu, Weiwei 
Jiang, Chaofan Wang, Kangning Yang, Jing Wei, Madeleine Antonellos, Emad Alghamdi, Prashan Madumal, Anam Khan, Wei Zhao, Toni McLennan, Elsy Garcia and many others. Thank you for being amazing company! I loved all our heart-to-heart chats, badminton games, board games and even the trips that we planned - but never happened!

I have a very special place in my heart for everyone who is involved with the Sri Lankan Graduates' Society (SLGS), at the University of Melbourne. I have had a wonderful time working with you for the past three years at various capacities. You are my family in Melbourne, and have been with me through the good times and the bad. I am lucky to have met many wonderful souls including, Malinga Perera, Chinthani Rathnayake, Shalanika Dayarathne, Erandathi Ekanayake, Udesh Gunarathna, Amila Silva, Gihan Pramuditha and many more. I wish you nothing but the very best for future endeavours.

Next, I would like to thank Prof. Martin Tomitsch, Assoc. Prof. Lian Loke, Dr. Joel Fredericks, Prof. Jeni Paay, Dr. Greg Wadley, Dr. Naseem Ahmadpour, Dr. Tuck Leong, Dr. Bernd Ploderer, Dr. Diego Munoz, Dr. Callum Parker, Assoc. Prof. Glenda Cadwell, Prof. Judy Kay, Dr. Sonja Pedell, Kadek Satriadi, Jessica Rahman and Ajit Pillai - with whom I worked with to organise OzCHI 2020. It was a very rewarding experience for me, and I enjoyed every bit of it! Thank you Dr. Sarah Webber for taking me on board as a Social media Co-chair for this event. I look forward to working with you again soon!

I am very thankful to the University of Melbourne for providing me with the Melbourne Research Scholarship. I also take this opportunity to thank the donors of the Rowden White Scholarship and the Diane Lemaire Scholarship for acknowledging and supporting my research. I would also like thank the students that I have tutored over the past three years. Your feedback and success contribute to a big part of my happiness as a tutor. I am also grateful to have mentored/supervised Stella Peng, Jolan $\mathrm{Hu}$ and Shangqian Li. Thank you for considering me as a person worthy of being your mentor/supervisor!

To several very special souls at the University of Moratuwa, Sri Lanka: none of this could have been possible without the foundation you laid during my bachelor's degree. Special thanks to Dr. Lochandaka Rathnayake, Dr. Supunmali Ahangama, Ms. Thilini Weerasuriya, Mr. B. Sudantha, Ms. Sumudu Wijethunge, Dr. Thanuja Sandanayake, Dr. Priyanga Talagala and others, who not only pushed me to do my best, but also continued to guide and support me in my PhD journey, all the way from Sri Lanka. I am truly blessed to have you as my teachers.

To my family and friends in Sri Lanka, I miss you dearly! I am incredibly thankful to my parents - Warna and Krishanthi Wijenayake - who taught me my ABCs, and treated my wins and failures as their own. I love you for believing in me, and for not letting me give up. I am also blessed to have a superwoman for a sister (Shashini) and a humble soul for a brother-in-law (Senura), who have added colour to my life in ways unknown to them. Finally, to Malinda Jayawardana, thank you for being the most amazing husband. I am truly grateful for your love, compassion and company. You make me a better person, and there are no words to thank you for all that you have sacrificed for me, and for my future.

Thank you for being an important part of my story!

Senuri Wijenayake | Melbourne, September 2021 


\section{Contents}

$\begin{array}{ll}\text { Abstract } & \text { i }\end{array}$

Declaration $\quad$ iii

$\begin{array}{lll}\text { Preface } & \text { v }\end{array}$

Acknowledgements vii

$\begin{array}{ll}\text { Contents } & \text { ix }\end{array}$

$\begin{array}{ll}\text { List of Tables } & \text { xi }\end{array}$

1 Introduction $\quad 1$

1.1 Motivation ................... 1

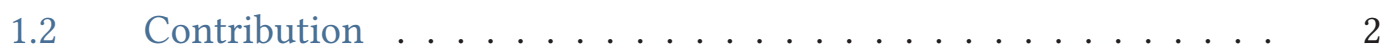

1.3 Thesis Outline ..................... 3

2 Background $\quad \mathbf{5}$

2.1 Social Conformity Concepts . . . . . . . . . . . . 5

2.2 Literature Selection . . . . . . . . . . . . . . 8

2.3 Overview of the Literature . . . . . . . . . . . . . 9

2.4 Contextual Determinants of Social Conformity . . . . . . . . . . 19

2.5 Personal Determinants of Social Conformity . . . . . . . . . 36

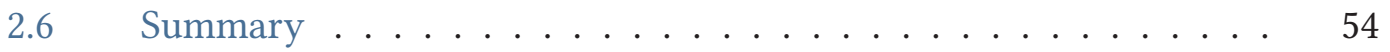

3 Methodology $\mathbf{5 5}$

$3.1 \quad$ Research Approach . . . . . . . . . . . . . . . . . 55

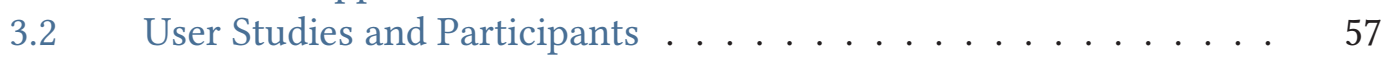

3.3 Ethical Considerations . . . . . . . . . . . . . . . . 59

3.4 Data Analysis . . . . . . . . . . . . . . . . . . 61

3.5 Limitations . . . . . . . . . . . . . . . . . . 62

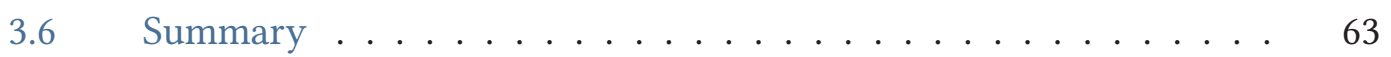

4 Contextual \& Personal Determinants of Online Social Conformity 65

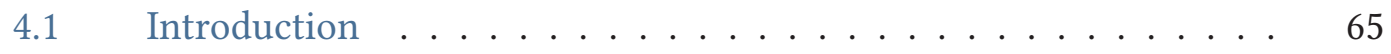

$4.2 \quad$ Article I . . . . . . . . . . . . . . 66

$\begin{array}{llr}5 & \text { Age as a Determinant of Conformity } & 89\end{array}$ 
$5.1 \quad$ Introduction . . . . . . . . . . . . . . . . 89

$5.2 \quad$ Article II . . . . . . . . . . . . . . . . . . . . . . 90

6 Gender as a Determinant of Conformity 113

6.1 Introduction . . . . . . . . . . . . . . . . 113

$6.2 \quad$ Article III . . . . . . . . . . . . . . . . . . . . . . . 114

$7 \quad$ Online Social Presence as a Determinant of Conformity 139

7.1 Introduction . . . . . . . . . . . . . . . 139

$7.2 \quad$ Article IV . . . . . . . . . . . . . . . . 140

$8 \quad$ Leveraging Conformity Influences for Prosocial Behaviours $\quad 163$

$8.1 \quad$ Introduction . . . . . . . . . . . . . . . . 163

$8.2 \quad$ Article $V \ldots \ldots \ldots \ldots \ldots$

9 Discussion $\quad \mathbf{1 7 3}$

9.1 Impact of Contextual Determinants on Online Social Conformity . 173

9.2 Impact of Personal Determinants on Online Social Conformity . . 175

9.3 Positive and Negative Outcomes of Online Social Conformity . . . 178

9.4 Accounting for Social Conformity in Platform Design . . . . . . 179

9.5 Future Directions of Online Social Conformity Research . . . . . . 181

$\begin{array}{llr}10 & \text { Conclusion } & 187\end{array}$

$\begin{array}{lr}\text { References } & 189\end{array}$ 


\section{List of Tables}

2.1 Overview of the research papers analysed. . . . . . . . . .

2.2 The distribution of conformity studies based on the determinants they 19

2.3 investigate. . . . . . . . . . . . . . . . 



\section{Chapter 1}

\section{Introduction}

Social conformity is a powerful social phenomenon that motivates individuals in group settings to adjust their personal judgements to agree with contradicting judgements of the group's majority [11,12]. Since the mid-20th century, researchers have investigated social conformity in physical groups, to understand its impact on how people form opinions in group settings. In a landmark study, Asch [11] investigated how being opposed by a clearly incorrect yet unanimous group majority affect personal judgements of individuals in a simple "line matching" task (matching a straight line with one of three other options based on length). They found that in such social pressure situations individuals often conformed to the incorrect majority, resulting in an astonishing $33.3 \%$ errors in judgement - in comparison to $1 \%$ of errors when completing the same task independently. Their findings established the presence of social conformity in physical groups, even when the group majority is clearly incorrect.

Subsequently, Deutsch and Gerard [42] explained two motives behind social conformity behaviour - informational and normative social influences. They rationalised that individuals conform either because they accept the majority's judgements to accurately reflect reality and use them to guide their personal judgements when the "correct" response is unclear (informational influences), or because they attempt to "fit in" by agreeing to the expectations of the group. Additionally, literature also describes social conformity as a function of several contextual (e.g., majority group size and task objectivity [20, 82]) and personal (e.g., gender, age, self-confidence and personality [39, $47,91,142]$ ) determinants, that impact the magnitude of its influence in physical groups.

\subsection{Motivation}

With the proliferation of societal interactions to computer-mediated communication (CMC) based group settings, understanding how social conformity influences affect our judgements in CMC platforms is crucial. However, given the inherent differences between physical and CMC group settings - e.g., anonymity and reduced social presence in the latter [118] - our understanding of social conformity based on physical groups is not sufficient for this purpose. Furthermore, while recent work shows that conformity manifests in diverse CMC groups such as learning platforms [19, 139], discussion forums and support groups [97, 154, 168], and social networks [32, 110,111], they do not adequately quantify the effects of the different contextual and personal determinants of social conformity (as observed in physical groups) in CMC settings. Additionally, it is unclear if and how factors that can moderate social conformity effects - but are implicit in face-to-face settings i.e., user cues and perceived social presence $[42,47,90]$ - would manifest differently in CMC groups. 
Moreover, contrary to observations in physical settings [42], social conformity can have both positive and negative outcomes in CMC groups. For instance, while some studies indicate that conforming to the majority's judgements results in fewer correct answers in group quizzes [19], as well as in groups of crowd workers completing visual judgement tasks together [81], more recent studies indicate that social conformity influences can encourage users of online support groups [154] and discussion forums [168] to follow accepted community standards and norms, leading to more positive sharing behaviour and an enhanced sense of belonging. Hence, these studies signify the potential for social conformity influences to enable prosocial behaviours in CMC group settings, given that its adverse effects (e.g. undue pressure to conform to majority's incorrect judgements) are mitigated. Nevertheless, these studies merely report positive and negative outcomes of conformity, and do not sufficiently frame these outcomes in relation to the presence of different contextual and/or personal determinants. Furthermore, the literature also indicates that social conformity influences can be manipulated through the design of CMC group platforms [168] - but does not offer concrete recommendations on how to achieve this.

\subsection{Contribution}

\subsubsection{Research Questions}

Based on the identified research gaps, this thesis aims to understand the dynamics of social conformity in CMC platforms in order to inform the design of future CMC group platforms that account for social conformity effects to ensure positive social interactions.

To this end, this work systematically investigates whether and how certain established contextual and personal determinants of social conformity in face-to-face groups, manifest in CMC group settings. This includes determinants that are especially relevant in CMC groups as a result of their lack of physicality and reduced social presence [118]. Specifically, this research explores the effects of age and gender cues, and social presence - all of which while obvious in a face-to-face context, are design decisions in CMC platforms. With this knowledge in hand, this thesis then examines potential positive and negative outcomes of social conformity in CMC groups, and proposes a set of design guidelines to inform how observed conformity effects can be controlled by manipulating the relevant conformity determinants. In particular, we set out to answer the following research questions:

RQ 1: What is the impact of established contextual determinants of face-to-face conformity literature (majority-minority group composition, task objectivity, and the level of social presence) in triggering social conformity in CMC groups?

RQ 2: What is the impact of established personal determinants of face-to-face conformity literature (gender, age, self-confidence and personality) in triggering social conformity in CMC groups?

RQ 2(a): How do gender and age based stereotypical perceptions of (peer and self) competency impact conformity behaviour in CMC groups? 
RQ 3: What are the potential positive and negative outcomes of online conformity?

RQ 4: How can we embed conformity determinants in the design of CMC group platforms to control conformity influences appropriately?

\subsection{Thesis Outline}

The rest of this thesis is organised as follows. Chapter 2 introduces the technical terminology and theoretical concepts often used in social conformity literature, and summarises prior findings on the contextual and personal conformity determinants that are analysed in this thesis. Next, Chapter 3 describes the experimental methodology used in the studies reported in the research articles (Article I-V).

Chapters 4, 5, 6, $7 \& 8$ present the five research articles that investigate the research questions of this thesis. More specifically, Chapter 4 describes a research study that investigates how established contextual and personal determinants of face-to-face social conformity impact user behaviour in an online group quiz (RQ 1 \& RQ 2). Chapter 5 presents a study that closely examines the effects of age and related stereotypes on the conformity behaviour of users who complete a set of stereotypical tasks through an Instant Messaging platform (RQ 2 \& RQ 2(a)), and describes how age-stereotypical conformity can be mitigated through appropriate platform design (RQ 4). Subsequently, Chapter 6 outlines another experiment that aims to investigate how effects of gender and related stereotypes manifest alongside other contextual and personal conformity determinants to impact user conformity in an online group quiz (RQ 1, RQ 2, \& RQ 2(a)). This study also quantifies the outcomes of gender-stereotypical conformity on the accuracy of user answers to the quiz questions (RQ 3), in addition to presenting a set of design guidelines to mitigate gender-stereotypical conformity in CMC groups (RQ 4).

Chapter 7 features a study that closely analyses how online social presence - a determinant that is more relevant in CMC groups than in physical settings - together with other contextual and personal conformity determinants, impact social conformity in a group quiz setting (RQ $1 \& \mathbf{R Q} 2)$. This study also details how three aspects of social presence - i.e., user representation, interactivity and response visibility - can be used to control perceived social presence in CMC platforms to control conformity influences (RQ 4). Next, Chapter 8 presents findings of an experiment that reports social conformity behaviour in how people perceive trustworthiness of news articles posted on online social media, and explains the study's findings with regard to effects of contextual and personal conformity determinants (RQ $1 \& \mathbf{R Q} 2)$. This study also highlights that social conformity impacts how people differentiate between, and subsequently respond to, fake and real news articles in social media settings - identifying potential outcomes of social conformity (RQ 3).

Chapter 9 reflects on the findings of this thesis and describes how they answer the research questions. Furthermore, informed by the findings of this thesis, this chapter also sets forth several avenues for future work in this area. Finally, Chapter 10 concludes with a summary of this thesis. 



\section{Chapter 2}

\section{Background}

This chapter presents findings of a review of the social conformity literature in both physical and CMC group settings, that informed the research questions of this thesis and the experimental design of the studies presented in Articles I-V. We first introduce the terminology and concepts that are vital to understand the social conformity literature in Section 2.1 - including how conformity behaviour is captured and rationalised in the literature, the differentiation between physical and CMC group settings, popular experimental paradigms used to investigate conformity, and how contextual and personal conformity determinants are characterised in literature. In Section 2.2 we then describe the methodology used for this review, including the criteria used for selecting and filtering prior work relevant to this thesis. Section 2.3 provides an overview of the literature analysed, and their distribution across physical and CMC group settings, experimental paradigms used, and contextual and personal determinants investigated. Next, Sections 2.4 and 2.5 provide a detailed look of findings of prior work with regard to contextual and personal determinants of social conformity respectively, emphasising on how these determinants have been manipulated, and their effects on social conformity behaviour in both physical and CMC groups.

\subsection{Social Conformity Concepts}

\subsubsection{Approaches and Motives}

Social conformity has been captured using two distinct approaches in the literature. In seminal studies using Asch's "line matching" task, conformity behaviour was determined by the number of times participants agreed with the erroneous judgement of the group majority (i.e., the social pressure condition), in comparison to participants' tendency to make errors when performing the same task independently (i.e., the control condition) [11, $12,42,141]$. However, more recent studies have attempted to capture an adjustment in individual opinion more specifically, by obtaining participants' responses to tasks both before and after exposing them to group feedback [61, 77, 110, 179, 180, 181, 182, 183, 186]. Both these approaches hold merit as they have been shown to successfully capture effects of social influences triggered by groups on how people form judgements. We further highlight that the experimental design of a typical conformity study contains three aspects (regardless of other factors investigated): 1) capturing opinions of individuals without social pressure, 2) exposing individuals to contradicting group opinions to create social pressure, and 3) capturing opinions of individuals after exposure to social pressure. Given the presence of these aspects, the experiment can then investigate effects of additional determinants (e.g., by changing the size of the opposing majority). 
Furthermore, the literature has been consistent on the two main motives behind conformity behaviour, namely normative influences and informational influences [42]. Normative influences manifest when individuals adjust their personal judgements to reflect that of their group, for the sole purpose of "fitting in". An example of normative conformity is the Asch's line judgement study [11], where individuals conformed to obviously incorrect judgements of the majority $33.3 \%$ of the times in a fairly simple task (only $1 \%$ error rate when individuals performed the same task in the absence of social pressure). Thus, conformity behaviour in Asch's study was fully attributed to normative influences. Similarly, normative influences have also been observed in CMC-based conformity studies where individuals tend to conform with group norms primarily to be accepted within social circles [154]. Conversely, people also tend to follow the crowd or choose the majority's opinion to improve their chances of being "correct" in ambiguous situations, as a result of informational influences. For example, conformity studies in CMC-based learning platforms indicate that students often tend to choose popular answers to quiz questions forsaking their own judgement, with the primary intention of obtaining more "correct" answers [19].

\subsubsection{Physical vs. Computer-Mediated Communication (CMC) Groups}

Being a consequence of group-oriented social pressure, conformity behaviour was initially investigated in physical groups. We describe a physical group as a gathering of two or more individuals working on a specific task, from a single location, such that every group member's physical presence is apparent to all others. A good example for a physical group is Asch's line matching experiment [11] where all group members were gathered around a table, where they could clearly see each other while indicating their responses vocally. Conversely, $C M C$ groups differ from physical groups in that they lack physicality (individuals can not physically see or communicate with each other) and that they facilitate communication through computer-mediated means i.e., text, audio, video, or a combination of them. The lack of physical connection results in lower social presence among CMC group members than in physical groups [118]. In addition to the lack of physicality, CMC groups offer the possibility for anonymity and asynchronous communication between group members - also leading to reduced perceived social presence [118]. For instance, users of real-world CMC groups often have the freedom to create user accounts without revealing their real name and photograph - giving them a sense of anonymity (e.g. Reddit $\left.{ }^{1}\right)$.

However, despite these differences, recent work has highlighted that conformity still manifests in a variety of CMC groups - including but not limited to - online learning platforms, social media, news sharing websites, and support groups [19, 110, 111, 154, 168]. In general, these studies are concerned with groups of individuals who communicate with others through web-based computer-mediated communication methods (e.g. textbased chats and posts on community forums), to achieve a certain group objective (e.g.

\footnotetext{
${ }^{1}$ https://www.reddit.com
} 
complete a quiz together) - thereby exposing them to group pressure situations similar to what can be found in physical groups.

\subsubsection{Experimental Paradigms}

The literature has leveraged two distinct paradigms in how group-based social influences have been induced when investigating conformity in physical and CMC groups namely Asch's paradigm and Crutchfield's paradigm. As their names indicate, the paradigms were inspired by the experimental setups used in two seminal conformity studies conducted by Asch [11] and Crutchfield [39] in the 1950s. These are two approaches in which an experimenter can simulate a group majority (or in other words, the majority's opinion) instead of allowing it to form organically within the group. Doing so, the experimenter can ensure the existence of a clear majority and control for the "majority's opinion" while also positioning the study participant(s) in the group's minority to induce group pressure.

Asch's paradigm was the first to be reported in 1951, in the pivotal line matching experiment [11]. This paradigm uses confederates of the experimenter who are instructed on how to respond to experimental tasks well before the experiment, to simulate a clear group majority in the presence of one or two real participants. The participants who are oblivious to this fact assumes all group members to be participants similar to themselves. In the beginning of the experiment, all group members are assigned a letter from the English alphabet (e.g., A-E) or a number (e.g., 1-5), that determines the order in which they will indicate their response - participant "A" first, participant " $B$ " second and so on. During the experimental task, each group member will be called to indicate their response verbally in an alphabetical/numerical order. The real participant is assigned the final position of the group, so that confederates can indicate their responses before the participant, thereby exposing the participant to the supposed "group's judgement" on the task.

Conversely, Crutchfield used an electrical panel to mimic group feedback, without using confederates [39]. In this approach each participant faces an electrical panel with multiple rows of lights bulbs - where each row (for instance, marked A-E) represents a group member, and the light bulbs in each row (for instance, marked 1-5) indicates the answer options. All participants are isolated in soundproof booths in the same location, and will use this setup to view responses of other group members and indicate their own response to an experimental task. For instance, if participant "A" chooses the 3rd answer option, the 3rd light bulb in the row corresponding to participant " $\mathrm{A}$ " will light up. Moreover, similar to Asch's paradigm, participants are instructed to indicate their answers based on the order of the letter/number assigned to them (e.g. A-E). However, all participants are led to believe they are in the final position (or participant "E"), while the experimenter simulates the responses of the remaining group members using the electrical setup. The setup is designed in such a way that the same set of "group responses" are displayed to all the participants through the panel, simultaneously. As a result, Crutchfield's paradigm can be used to investigate conformity behaviour in multiple participants simultaneously, while also exposing them to the same group pressure situation, in contrast to Asch's paradigm. 
While both paradigms are rooted in literature related to physical groups (with all participants/confederates in one room), these approaches can be implemented in CMC groups as well. On one hand, using confederates who communicate via computer-mediated means to investigate how people respond to social pressure situations, mimics the Asch's paradigm (e.g. Laporte, Nimwegen, and Uyttendaele [97] investigated conformity in a CMC group where one participant and five confederates communicated through live video or text-based chat to complete an online quiz together). On the other hand, if group feedback displayed to participants is computer-simulated (e.g., as a bar chart illustrating votes against each answer option [139], automated peer responses [89, 130]), and exposes participants to the same group pressure situation without using confederates, it resonates with Crutchfield's paradigm.

\subsubsection{Determinants of Conformity}

We refer to a factor that directly influences (encourages or reduces) conformity behaviour as a "determinant" of social conformity. We broadly categorise conformity determinants as either contextual or personal. Contextual determinants form the external social pressure situation that triggers conformity behaviour from the participant involved. For instance, the number of group members opposing participant's personal judgement, the nature of the experimental task, and perceived social presence are critical contextual determinants of conformity $[11,42]$. Conversely, personal determinants - i.e., age, gender, personality, and self-confidence on the experimental task - relate to individuals, and can determine their susceptibility to social conformity influences. Such personal factors can not only vary among participants of a social pressure situation, but also between the group members inflicting the said social pressure.

\subsection{Literature Selection}

We performed a thorough review of social conformity research conducted in both physical and CMC-based settings. To this end, we chose four digital libraries that contain venues that explore socio-psychological phenomena in both physical and CMC groups, namely - Wiley, American Psychological Association (APA) and Taylor \& Francis for conformity literature on physical groups, and Association for Computing Machinery (ACM) for conformity research based on CMC groups. In addition, we included the scholarly journal Computers in Human Behavior in our analysis of conformity research based in CMC groups - given its commitment to investigate the role of computers in social psychology. We further highlight that due to the overwhelming number of social influence studies published in Wiley, APA and Taylor \& Francis libraries, we then narrowed our search to several prominent social psychology journals in each library namely - fournal of Applied Social Psychology, British fournal of Social Psychology and European fournal of Social Psychology in Wiley, Journal of Applied Social Psychology and Journal of Personality and Social Psychology in APA and The Journal of Social Psychology, Social Influence, and International fournal of Psychology in Taylor \& Francis. 
We used the following query strings to retrieve conformity research based on physical and CMC settings separately. We note that two slightly different query strings were used for the two groups of venues (that focus on physical vs. CMC groups), to adopt differences in terminology used in conformity research in each context and minimise false positives.

- For studies based on physical settings: "social conformity OR (normative AND (conformity OR influence OR social influence)) OR (informational AND (conformity OR influence OR social influence))"

- For studies based on CMC settings: "social conformity OR conformity OR normative influence OR normative conformity OR informational influence OR informational conformity OR (social influence AND (normative OR informational))"

Furthermore, as social conformity has been investigated since early 1950s we applied a search filter to retrieve publications from January 1950 till July 2021 - which accounts for over 70 years of conformity literature. To ensure that only the most relevant publications were retrieved, we restricted our search to metadata (e.g., paper title, abstract and keywords) and only considered complete research articles (i.e., short papers, extended abstracts, review papers, posters, demonstrations, editorials, book chapters, and work-inprogress were not considered).

We retrieved a total of 484 papers through the aforementioned process. We excluded papers that investigate other forms of social influence (e.g. theory of planned behaviour, exchange theory), discuss effects of social influences without empirical evidence (or has no evaluation), and studies that employ surveys to simply measure tendency to conform. As a result, 70 papers that either report a manifestation of conformity or presents a study specifically designed to investigate contextual and/or personal determinants of conformity in physical or CMC-based groups were retained for further analysis.

Furthermore, to ensure that a good proportion of the relevant conformity research is included in the final sample, we then hand-picked seminal research papers that report on conformity determinants, but are published in digital libraries which were not considered for the initial assessment. These papers were included in the final sample based on their relevance to the thesis objectives. This process resulted in an additional 40 complete research articles, published in FSTOR, Elsevier, Sage, Science, IEEE, Springer and in APA (journals other than the two considered before). Thus, the final sample considered eligible for further analysis included a total of 110 full research papers.

\subsection{Overview of the Literature}

We present a complete list of papers considered for the review $(n=110)$ and summarise several key characteristics of these research studies - such as their setting, experimental paradigm, observed conformity rate, and investigated contextual and/or personal conformity determinants - in Table 2.1. We also present this information for the studies presented in Chapters 4, 5, 6, $7 \& 8$ at the end of Table 2.1 for ease of reference and comparison. 
Table 2.1: Overview of the research papers analysed.

\begin{tabular}{|c|c|c|c|c|c|c|c|c|c|c|}
\hline \multirow[t]{2}{*}{ Reference } & \multirow[t]{2}{*}{ Setting } & \multirow[t]{2}{*}{ Paradigm } & \multirow[t]{2}{*}{$\begin{array}{l}\text { Conformity } \\
\text { Rate }\end{array}$} & \multicolumn{3}{|c|}{ Contextual } & \multicolumn{4}{|c|}{ Personal } \\
\hline & & & & 产 & 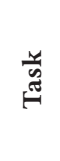 & 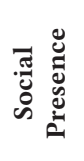 & 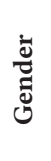 & $\stackrel{8}{<}$ & $\begin{array}{l}\tilde{\Xi} \\
\tilde{\Xi} \\
\tilde{\Xi} \\
\tilde{\Xi}\end{array}$ & 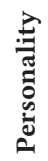 \\
\hline Asch [11] & Physical & Asch & $5.5-33.3 \%$ & * & & & & & & \\
\hline Goldberg [64] & Physical & Crutchfield & $30 \%$ & * & & & & & & \\
\hline Crutchfield [39] & Physical & Crutchfield & $38.1 \%$ & & * & & * & * & & * \\
\hline Deutsch and Gerard [42] & Physical & Both & $1.9-57.2 \%$ & & * & * & & & & \\
\hline Asch [12] & Physical & Asch & $9-36.8 \%$ & * & & & & & & \\
\hline $\begin{array}{l}\text { Mouton, Blake, and Olm- } \\
\text { stead [123] }\end{array}$ & Physical & Crutchfield & - & * & & * & & & & * \\
\hline $\begin{array}{l}\text { Blake, Helson, and Mou- } \\
\text { ton [20] }\end{array}$ & Physical & Crutchfield & $27.4-41.5 \%$ & & * & & & & & \\
\hline Hardy [72] & Physical & Asch & $45 \%$ & * & & & & & & * \\
\hline $\begin{array}{l}\text { Coleman, Blake, and Mou- } \\
\text { ton [31] }\end{array}$ & Physical & Crutchfield & $10-60 \%$ & & * & & * & & & \\
\hline Beloff [18] & Physical & Crutchfield & - & & * & & * & & & * \\
\hline McDavid Jr [114] & Physical & Crutchfield & $36.5-60 \%$ & & * & & & & & * \\
\hline Smith [160] & Physical & Asch & - & & & & & & * & * \\
\hline Rosenberg [141] & Physical & Crutchfield & - & * & & & & & & \\
\hline $\begin{array}{l}\text { Strickland and Crowne } \\
\text { [167] }\end{array}$ & Physical & Crutchfield & $34.2-56.1 \%$ & & & & & & & * \\
\hline Rosenberg [142] & Physical & Crutchfield & - & * & & & & & * & \\
\hline Crowne and Liverant [37] & Physical & Asch & $30.8-61.1 \%$ & & & & & & * & * \\
\hline Allen and Crutchfield [3] & Physical & Crutchfield & $21.2-69.8 \%$ & & * & & * & & & \\
\hline Gerard [60] & Physical & Both & - & & & * & & & & \\
\hline $\begin{array}{l}\text { Sistrunk and McDavid } \\
\text { [157] }\end{array}$ & Physical & Crutchfield & - & & * & & * & & & * \\
\hline Costanzo and Shaw [36] & Physical & Crutchfield & $28.1-51.9 \%$ & & & & * & * & & \\
\hline Steiner and Vannoy [166] & Physical & Asch & - & & & & & & & * \\
\hline Goldberg and Rorer [63] & Physical & Crutchfield & $30 \%$ & & & & & & & \\
\hline McGhee and Teevan [115] & Physical & Crutchfield & $35.7-43 \%$ & & & & & & & * \\
\hline Meunier and Rule [119] & Physical & Crutchfield & $11.1-26.3 \%$ & & & & & & * & * \\
\hline Schulman [153] & Physical & Crutchfield & $22-57.7 \%$ & & & & * & & & \\
\hline Gergen and Bauer [61] & Physical & Crutchfield & - & & * & & & & * & * \\
\hline
\end{tabular}


Table 2.1 - Continued from previous page

\begin{tabular}{|c|c|c|c|c|c|c|c|c|c|c|}
\hline \multirow[t]{2}{*}{ Reference } & \multirow[t]{2}{*}{ Setting } & \multirow[t]{2}{*}{ Paradigm } & \multirow{2}{*}{$\begin{array}{l}\text { Conformity } \\
\text { Rate }\end{array}$} & \multicolumn{3}{|c|}{ Contextual } & \multicolumn{4}{|c|}{ Personal } \\
\hline & & & & 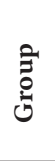 & 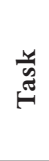 & 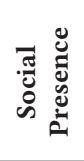 & $\begin{array}{l}\dot{\Xi} \\
\dot{J} \\
心\end{array}$ & $\ddot{\infty}$ & 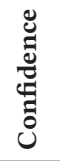 & 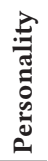 \\
\hline Allen and Levine [7] & Physical & Crutchfield & - & * & * & & * & & & \\
\hline Allen and Levine [4] & Physical & Crutchfield & - & * & * & & * & & & \\
\hline Costanzo [34] & Physical & Crutchfield & $33-50 \%$ & & & & & * & & * \\
\hline Allen and Levine [6] & Physical & Crutchfield & - & * & * & & * & & & \\
\hline Allen and Levine [5] & Physical & Crutchfield & - & * & * & * & * & & & \\
\hline Klein [91] & Physical & Crutchfield & $2.8-51.3 \%$ & & * & & * & * & & \\
\hline Allen and Newtson [8] & Physical & Crutchfield & $3.3-86.7 \%$ & * & * & & * & * & & \\
\hline $\begin{array}{l}\text { Endler, Minden, and North } \\
\text { [52] }\end{array}$ & Physical & Crutchfield & - & & * & & * & & & * \\
\hline Sistrunk [158] & Physical & Crutchfield & $24-35 \%$ & & & * & & & & * \\
\hline Larsen [98] & Physical & Asch & $26.4 \%$ & & & & * & & & \\
\hline $\begin{array}{l}\text { Endler, Coward, and } \\
\text { Wiesenthal [51] }\end{array}$ & Physical & Crutchfield & $25-44.8 \%$ & & * & & * & * & * & \\
\hline Endler et al. [53] & Physical & Crutchfield & $21.1-37.9 \%$ & & * & & * & & * & \\
\hline Morris and Miller [122] & Physical & Crutchfield & - & * & * & & & & & \\
\hline Duval [45] & Physical & Crutchfield & $29-53 \%$ & & & * & & & & \\
\hline Stang [164] & Physical & Crutchfield & $39-80 \%$ & * & & & & & & \\
\hline $\begin{array}{l}\text { Ross, Bierbrauer, and Hoff- } \\
\text { man [144] }\end{array}$ & Physical & Asch & $9.6-25.7 \%$ & & & & * & & * & \\
\hline Larsen et al. [99] & Physical & Asch & $13.5-37.5 \%$ & & & & * & & & * \\
\hline Perrin and Spencer [135] & Physical & Asch & $0-39 \%$ & * & & & & & & \\
\hline $\begin{array}{l}\text { Eagly, Wood, and Fish- } \\
\text { baugh [48] }\end{array}$ & Physical & Crutchfield & - & & & * & * & & & \\
\hline Santee and Maslach [148] & Physical & Crutchfield & $56.4-69.7 \%$ & * & & & * & & & * \\
\hline Kumar [94] & Physical & Asch & - & * & & & & * & & \\
\hline $\begin{array}{l}\text { Tesser, Campbell, and } \\
\text { Mickler [169] }\end{array}$ & Physical & Crutchfield & - & * & & & & & & * \\
\hline Mugny [124] & Physical & Crutchfield & $19.6-38.3 \%$ & * & & & & & & * \\
\hline Insko et al. [82] & Physical & Asch & $39.7-67.8 \%$ & * & & * & & & & \\
\hline $\begin{array}{l}\text { Nicholson, Cole, and Rock- } \\
\text { lin [126] }\end{array}$ & Physical & Asch & $4.8-15.5 \%$ & & & & & & & \\
\hline Eagly and Chrvala [47] & Physical & Crutchfield & - & & & * & * & * & & \\
\hline
\end{tabular}


Table 2.1 - Continued from previous page

\begin{tabular}{|c|c|c|c|c|c|c|c|c|c|c|}
\hline \multirow[t]{2}{*}{ Reference } & \multirow[t]{2}{*}{ Setting } & \multirow[t]{2}{*}{ Paradigm } & \multirow[t]{2}{*}{$\begin{array}{l}\text { Conformity } \\
\text { Rate }\end{array}$} & \multicolumn{3}{|c|}{ Contextual } & \multicolumn{4}{|c|}{ Personal } \\
\hline & & & & है & $\begin{array}{l}\frac{y}{\tilde{w}} \\
\tilde{\pi}\end{array}$ & 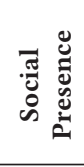 & $\frac{\dot{v}}{\tilde{J}}$ & $\underset{<}{2}$ & 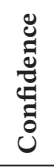 & 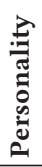 \\
\hline Wood and Karten [185] & Physical & Other & - & & & & * & & & \\
\hline $\begin{array}{l}\text { Yarnold, Grimm, and } \\
\text { Mueser [187] }\end{array}$ & Physical & Asch & $25-50 \%$ & & & & & & & * \\
\hline $\begin{array}{l}\text { Maslach, Santee, and Wade } \\
\text { [112] }\end{array}$ & Physical & Crutchfield & - & * & & & * & & & * \\
\hline McKelvey and Kerr [117] & Physical & Asch & $3-43 \%$ & * & * & * & & & & \\
\hline Nemeth and Chiles [125] & Physical & Asch & $70.4-14.1 \%$ & * & & & & & & \\
\hline $\begin{array}{l}\text { Smilowitz, Compton, and } \\
\text { Flint [159] }\end{array}$ & $\mathrm{CMC}$ & Crutchfield & $6.3 \%$ & & & & & & & \\
\hline Campbell and Fairey [28] & $\mathrm{CMC}$ & Crutchfield & - & * & * & * & & & & \\
\hline Spears, Lea, and Lee [162] & CMC & Crutchfield & - & & & * & & & & \\
\hline Abrams et al. [1] & Physical & Asch & $8.3-58.1 \%$ & & & * & & & & \\
\hline Rugs and Kaplan [145] & Physical & Crutchfield & - & & & & * & & & \\
\hline Walker and Andrade [175] & Physical & Asch & $0-85 \%$ & & & & & * & & \\
\hline $\begin{array}{l}\text { Schneider and Watkins } \\
\text { [151] }\end{array}$ & Physical & Other & $23 \%$ & & & & & & & \\
\hline $\begin{array}{l}\text { Baron, Vandello, and Brun- } \\
\text { sman [16] }\end{array}$ & Physical & Asch & $13-60 \%$ & & * & & * & & * & \\
\hline Pasupathi [132] & Physical & Crutchfield & $26-50 \%$ & & * & & & * & & \\
\hline Postmes et al. [136] & $\mathrm{CMC}$ & Other & - & & & * & & & & \\
\hline $\begin{array}{l}\text { Sassenberg and Postmes } \\
{[150]}\end{array}$ & $\mathrm{CMC}$ & Crutchfield & - & & & * & & & & \\
\hline Walther et al. [176] & $\mathrm{CMC}$ & Crutchfield & - & * & * & & & & & \\
\hline Lee and Nass [106] & $\mathrm{CMC}$ & Crutchfield & - & * & & * & & & & \\
\hline $\begin{array}{l}\text { Guadagno and Cialdini } \\
\text { [68] }\end{array}$ & Both & Asch & - & & & * & * & & & \\
\hline Lee [101] & $\mathrm{CMC}$ & Crutchfield & $32-46 \%$ & & * & & * & & & \\
\hline Lee [102] & $\mathrm{CMC}$ & Crutchfield & $26.9-58.6 \%$ & & * & & * & & * & \\
\hline Lee [103] & $\mathrm{CMC}$ & Crutchfield & - & & & * & & & & \\
\hline Reysen [137] & CMC & Asch & - & & & & & & & \\
\hline Lee [104] & CMC & Crutchfield & - & & & * & * & & & * \\
\hline Cinnirella and Green [29] & Both & Both & - & & & * & & & & \\
\hline
\end{tabular}


Table 2.1 - Continued from previous page

\begin{tabular}{|c|c|c|c|c|c|c|c|c|c|c|}
\hline \multirow[t]{2}{*}{ Reference } & \multirow[t]{2}{*}{ Setting } & \multirow[t]{2}{*}{ Paradigm } & \multirow{2}{*}{$\begin{array}{l}\text { Conformity } \\
\text { Rate }\end{array}$} & \multicolumn{3}{|c|}{ Contextual } & \multicolumn{4}{|c|}{ Personal } \\
\hline & & & & 产 & 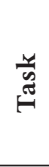 & 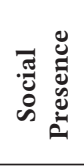 & 离 & $\ddot{\&}$ & $\begin{array}{l}\tilde{U} \\
\tilde{\Xi} \\
\tilde{E} \\
\tilde{E}\end{array}$ & 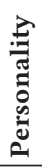 \\
\hline $\begin{array}{l}\text { Guadagno and Cialdini } \\
\text { [69] }\end{array}$ & Both & Asch & - & & & * & * & & & \\
\hline Lee [105] & $\mathrm{CMC}$ & Crutchfield & $41-59.6 \%$ & & * & * & * & & * & * \\
\hline $\begin{array}{l}\text { Laporte, Nimwegen, and } \\
\text { Uyttendaele [97] }\end{array}$ & $\mathrm{CMC}$ & Asch & $0-33 \%$ & & * & * & & & & \\
\hline Fard [55] & Physical & Asch & - & & & & * & & & \\
\hline Mori and Arai [121] & Physical & Asch & $5-28.6 \%$ & & & & * & & & \\
\hline Packer [130] & $\mathrm{CMC}$ & Crutchfield & - & & & & & & & * \\
\hline Kim and Park [89] & CMC & Crutchfield & - & & & * & & & & \\
\hline $\begin{array}{l}\text { Hullman, Adar, and Shah } \\
\text { [81] }\end{array}$ & $\mathrm{CMC}$ & Crutchfield & - & & & & & & & \\
\hline $\begin{array}{l}\text { Rosander and Eriksson } \\
{[139]}\end{array}$ & $\mathrm{CMC}$ & Crutchfield & $13 \%$ & * & * & & * & & & \\
\hline $\begin{array}{l}\text { Zhu, Huberman, and Luon } \\
\text { [188] }\end{array}$ & $\mathrm{CMC}$ & Crutchfield & $14.1-32.5 \%$ & * & * & & * & * & & \\
\hline Kraemer [92] & $\mathrm{CMC}$ & Asch & $50 \%$ & & & & & & & \\
\hline Kundu and Cummins [95] & Physical & Asch & - & & & & & & & \\
\hline Brandstetter et al. [24] & Physical & Asch & $1-59 \%$ & & * & * & & & & \\
\hline Maruyama et al. [111] & $\mathrm{CMC}$ & Other & $7-33 \%$ & & & * & & & & \\
\hline $\begin{array}{l}\text { Midden, Ham, and Baten } \\
{[120]}\end{array}$ & $\mathrm{CMC}$ & Asch & $0-24 \%$ & & * & * & & & & \\
\hline Beran et al. [19] & $\mathrm{CMC}$ & Asch & $46.9 \%$ & & & & * & & & \\
\hline Shiomi and Hagita [155] & Physical & Asch & $41-47 \%$ & & & * & & & & \\
\hline Hertz and Wiese [77] & $\mathrm{CMC}$ & Crutchfield & $22.3 \%$ & & * & * & & & & \\
\hline $\mathrm{Xu}$ and Lombard [186] & $\mathrm{CMC}$ & Crutchfield & - & & & * & & & & \\
\hline Maruyama et al. [110] & $\mathrm{CMC}$ & Crutchfield & - & & & * & & & & \\
\hline Salomons et al. [146] & Physical & Asch & $29 \%$ & & & & & & & \\
\hline $\begin{array}{l}\text { Kyrlitsias and Michael } \\
\text { Grigoriou [96] }\end{array}$ & $\mathrm{CMC}$ & Asch & $1.14-1.68 \%$ & & & * & & & * & \\
\hline Vollmer et al. [172] & Physical & Asch & $74-83 \%$ & & & * & & * & & \\
\hline Hertz and Wiese [78] & $\mathrm{CMC}$ & Crutchfield & $35.6-47.2 \%$ & & * & * & & & & \\
\hline Daniel and Peter [40] & Physical & Asch & $8-38 \%$ & * & & * & & & & * \\
\hline
\end{tabular}


Table 2.1 - Continued from previous page

\begin{tabular}{|c|c|c|c|c|c|c|c|c|c|c|}
\hline \multirow[t]{2}{*}{ Reference } & \multirow[t]{2}{*}{ Setting } & \multirow[t]{2}{*}{ Paradigm } & \multirow{2}{*}{$\begin{array}{l}\text { Conformity } \\
\text { Rate }\end{array}$} & \multicolumn{3}{|c|}{ Contextual } & \multicolumn{4}{|c|}{ Personal } \\
\hline & & & & 言 & 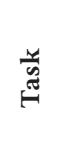 & 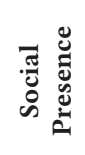 & 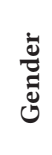 & : & 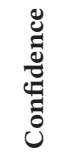 & 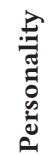 \\
\hline Perfumi et al. [134] & $\mathrm{CMC}$ & Crutchfield & $1.4-29.8 \%$ & & * & * & & & & * \\
\hline Lucas et al. [108] & $\mathrm{CMC}$ & Asch & - & & & * & & & & \\
\hline Colliander [32] & $\mathrm{CMC}$ & Crutchfield & - & & & & & & & \\
\hline Goodmon et al. [66] & Physical & Asch & $46 \%$ & & & & & & * & \\
\hline Salomons et al. [147] & Physical & Asch & $5.5-32.2 \%$ & & & * & & & & \\
\hline Bleize et al. [22] & $\mathrm{CMC}$ & Crutchfield & - & & & * & & & & * \\
\hline Schneider [152] & $\mathrm{CMC}$ & Crutchfield & $69.4-87.6 \%$ & & & & * & & & \\
\hline $\begin{array}{l}\text { Zonca, Folso, and Sciutti } \\
\text { [189] }\end{array}$ & $\mathrm{CMC}$ & Asch & - & & & * & & & & \\
\hline Chapter 4 & $\mathrm{CMC}$ & Crutchfield & $20.33 \%$ & * & * & & * & & * & * \\
\hline Chapter 5 & $\mathrm{CMC}$ & Asch & $28.4 \%$ & & * & & * & * & * & \\
\hline Chapter 6 & $\mathrm{CMC}$ & Crutchfield & $39 \%$ & * & * & & * & & * & \\
\hline Chapter 7 & $\mathrm{CMC}$ & Asch & $30 \%$ & * & * & * & * & & * & \\
\hline Chapter 8 & $\mathrm{CMC}$ & Crutchfield & $58 \%$ & * & & & * & & * & \\
\hline
\end{tabular}

\subsubsection{Physical vs. CMC groups}

During the preliminary analysis of the final sample of research articles we identified 73 research articles related to conformity research based on physical groups, and another 34 research articles based on CMC groups. The remaining 3 research articles (i.e., [29, $68,69]$ ) investigate conformity in both physical and CMC-based group settings, in an attempt to compare effects of social conformity in the two contexts. We then analysed the distribution of these physical and CMC-based conformity studies based on their publication years as illustrated in Figure 2.1. We note that while the sample included papers from 1951-2021, the earliest report of a CMC-based conformity study was in 1988 by Smilowitz, Compton, and Flint [159]. We also observe an increasing trend in the number of CMC-based conformity studies in the last two decades - indicating a rapidly increasing interest in conformity studies based on CMC groups.

\subsubsection{Experimental Paradigms}

Based on how the "majority's opinion" has been generated to induce a social pressure situation (as described in Section 2.1.3), we categorised the conformity studies reviewed into Asch's paradigm (uses confederates to create a majority) or Crutchfield's paradigm (simulated a majority without using confederates). Accordingly, we identified 39 papers 


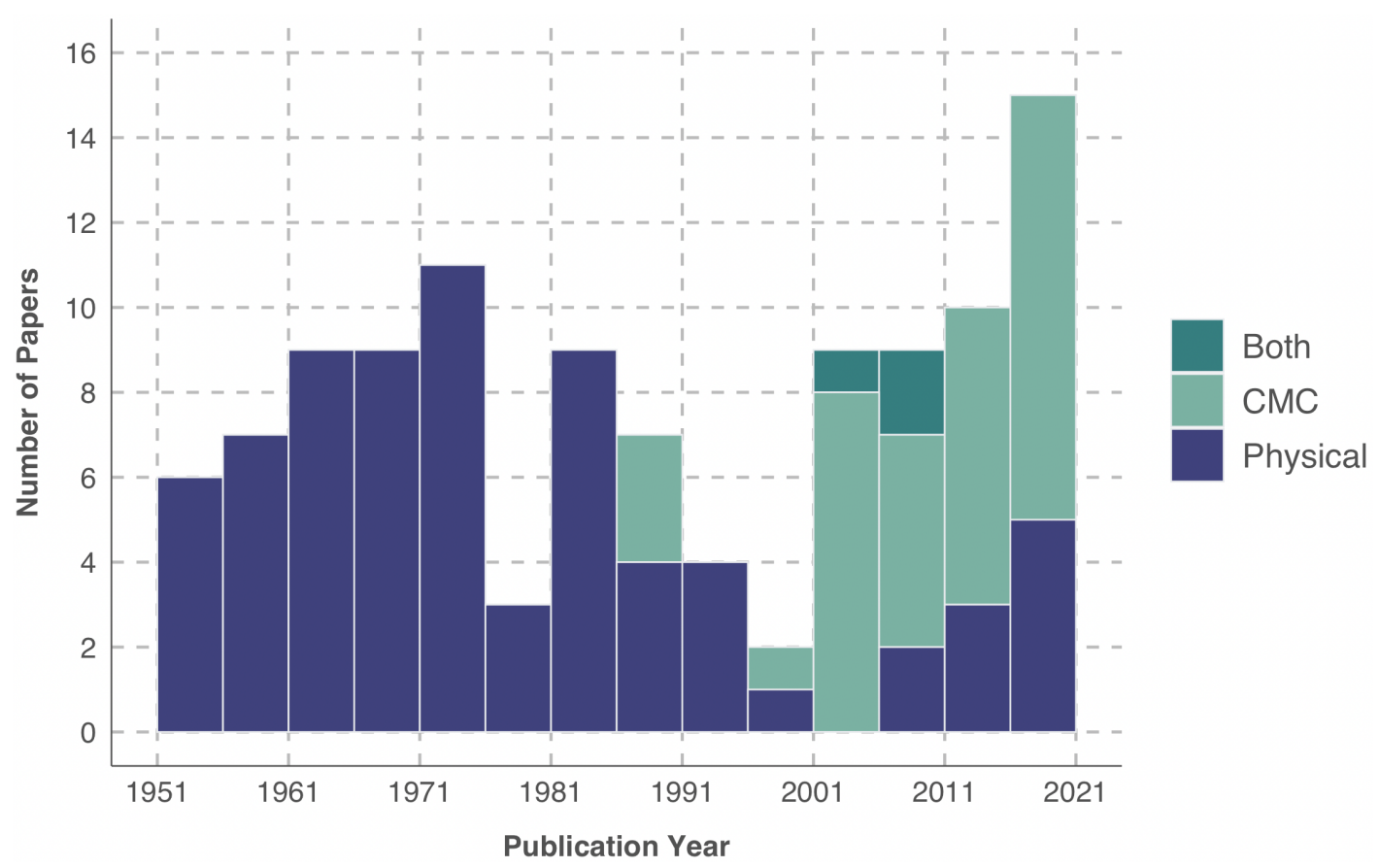

Figure 2.1: Distribution of the conformity studies based on CMC and physical groups considered in this review from 1951-2021.

(35.5\% of the total sample) that used Asch's paradigm (Physical: 29, CMC: 8, Both: 2). The majority of research studies that used Asch's paradigm in physical groups, describe a setup similar to what Asch used in 1951 [11]. More specifically, confederates who were previously instructed on how to respond to experimental tasks were inserted into the group alongside the real participant, to gain control over the group majority's response (see Section 2.1.3). However, these studies were not restricted to the line judgement task used by Asch [11]. We note that both objective tasks (e.g., indicating the longer tone out of two tones [144], or whether a spot of light has moved in each trial [117]), as well as subjective tasks (e.g., indicating response to hypothetical moral dilemmas [95]) were used in studies based on Asch's paradigm in physical groups.

Similarly, whenever confederates are connected through computer-mediate communication channels to create a group, the experimental setup follows Asch's paradigm in CMC settings. While in CMC groups, confederates themselves are not physically present in the same location as the participant, they are visible to the participant through various channels such as a real-time text-based chat (e.g. [19, 137]), a live video stream (e.g. [97, $108,120])$, or through virtual reality where each member is represented using an avatar (e.g. $[92,96])$. Similar to physical groups, in addition to the traditional line judgement task, a wide variety of experimental tasks (e.g., surveys with multiple choice questions on general knowledge, subject matter, moral opinions [19, 97], memory tasks [137]) have been used to investigate conformity in CMC-based groups using Asch's paradigm.

Moreover, $58.2 \%$ of the total sample considered for this survey $(n=64)$ have used Crutchfield's experimental paradigm (Physical: 40, CMC: 24). We note that in addition to Crutchfield's original electrical apparatus, the "group" has been simulated in physical 
groups by exposing participants who are isolated in soundproof booths in the same location, to tape recorded responses of others that are played sequentially with random intervals between them, before indicating their own responses to tasks [20,123]. Similarly, Crutchfield's paradigm shows many variations in CMC groups, where simulations are comparatively easier to implement than in physical groups. Hence, in addition to using a computer program to display predetermined responses to an experimental task [159], the "group responses" have been simulated by displaying the specific number of "peers" who support each response option [188], using fabricated bar charts to illustrate the proportion of "peer" responses supporting each response option [139, 162], and even by displaying automated textual responses accompanied by anthropomorphic avatars [103].

Interestingly, we note that out of all the articles reviewed in this survey, only three research articles (2.7\% of the total sample) have used both Asch's and Crutchfield's paradigms (as different experimental conditions). For example, Deutsch and Gerard [42] replicated the line judgement task using both Asch's and Crutchfield's paradigms to compare effects of perceived social presence on conformity behaviour in face-to-face and anonymous physical groups, respectively. Furthermore, four other research articles $(3.6 \%$ of the total sample) have investigated conformity behaviour when group composition is allowed to manifest organically, and hence did not use confederates (Asch's paradigm) or simulations (Crutchfield's paradigm) to generate social pressure. For instance, Wood and Karten [185] used face-to-face, mixed-gender groups of four participants each, to examine effects of gender differences in perceived self and peer competency on conformity behaviour in a group discussion task. Similarly, in a CMC-based study, Maruyama et al. [111] exposed participants to a naturally occurring Twitter dialogue by instructing them to follow a widely used Twitter hashtag related to a political debate, on which they were later asked to indicate their own opinion.

\subsubsection{Asch vs. Crutchfield}

Upon our initial analysis of the experimental paradigms used in conformity research, we performed a chi-square test of independence [116] to examine whether a statistically significant association exists between the nature of the group setting (physical or CMC) and the experimental paradigm used (Asch's or Crutchfield's). For this analysis, 9 research articles that used both paradigms $(n=3)$, used no simulations $(n=4)$, or investigated conformity in both physical and CMC-based groups $(n=2)$ were not considered. We found no significant association between the two variables using a chi-square test $\left(\chi^{2}(1,101)=2.73, p=.10\right)$. In other words, the proportion of studies that report using either one of the paradigms did not differ based on the physical/CMC-based nature of the group setting. We note that regardless the physical/CMC context of the group setting, Crutchfield's paradigm is more frequently used in conformity studies $(55 \%$ of physical, and $70 \%$ of CMC-based studies), than Asch's paradigm (39\% of physical, and $24 \%$ of CMC-based studies).

The primary reason why Crutchfield's paradigm may be more preferable in certain situations could be its ability to generate a consistent group pressure situation with less resources (no requirement for confederates), that allows testing multiple participants simultaneously. Meanwhile, Asch's paradigm is often perceived to be "handicapped 
by the severely unfavourable ratio of confederates to true subjects" [39]. This can be especially tedious in physical groups, where researchers go to great lengths to ensure that the confederates are adequately trained and prepared to provide the appropriate response, and are seated in the correct order (so that the participant is in the last seat).

Simulating group responses is also seen to provide more control over what information is exposed to participants in a social pressure situation, so that confounding influences can be mitigated $[20,139]$. This is especially important in studies that investigate effects of conformity determinants other than majority group size - i.e., nature and difficulty of task, personality of participant etc. For instance, several conformity studies that examined effects of the experimental task (objectivity and difficulty) and participants' personality on conformity behaviour in physical [20, 157] and CMC settings [139] used Crutchfield's paradigm to ensure that non verbal user cues (e.g. peer gender/age) do not influence participants' decision to conform to the majority. More specifically, the aforementioned CMC-based studies used fabricated bar charts to simulate the proportion of "peer" responses supporting each response option (e.g., 90\% chose response option A, $10 \%$ chose response option B), to purposefully avoid exposing personal cues of "peers" to minimise confounding effects - which is almost impossible to prevent in Asch's paradigm.

On the other hand, Crutchfield's paradigm is also used in CMC-based conformity studies that investigate effects of certain personal attributes of "peers" who generate social pressure $[102,105]$. These studies have used stereotypically gendered human-like avatars and other user representations to identify simulated "peers", as they indicate their responses on experimental tasks. Therefore, in addition to being able to cater to a wide variety of experimental tasks in both physical and CMC groups (as is with Asch's paradigm), Crutchfield's paradigm is versatile with different experimental objectives with regard to conformity studies.

Moreover, in CMC-based studies where conformity was identified as a change in initial and final responses after being exposed to "group feedback", researchers have used computer software to dynamically simulate an opposing majority, based on the initial response of the participant (e.g. if participant chose option A, the simulated majority will support option B and vice versa). This approach ensures that regardless the initial responses of participants, they all face the same set of social pressure situations. For example, in an attempt to quantify effects of the opposing majority's group size $(2 \mathrm{x}, 5 \mathrm{x}, 10 \mathrm{x}, 20 \mathrm{x}$ the size of the minority) on conformity behaviour in a preferencebased task, Zhu, Huberman, and Luon [188] displayed a specific number of "others" who supposedly chose each of the two options available (e.g., 150 chose A and 15 chose B, or 40 chose A and 80 chose B). The experimental setup used was not only able to dynamically simulate the group responses, so that the simulated majority was often challenging the initial choice of the participant, but also varied the majority and minority group sizes as per the experiment's objectives. Thus, Crutchfield's paradigm can be used to dynamically simulate social pressure conditions - especially when the participant's personal response/judgement (e.g. preference out of two options) is difficult to be predetermined - unlike the line judgement task, where participants can be reasonably expected to select the "correct" answer.

However, despite all the aforementioned advantages of Crutchfield's paradigm, Asch's paradigm is still regularly used in both physical [66, 172] and CMC-based [97, 120] 
conformity studies - especially when investigating effects of normative influences on social conformity. The social pressure situation created in Asch's paradigm - where a participant's personal judgement is contradicted by a clearly incorrect group majority triggers conformity mostly as the participant attempts to "fit in" with others (normative influence), and rarely because they cannot recognise the correct response to the task at hand (informational influence). Therefore, in recent CMC studies, Asch's paradigm has been used to investigate effects of different levels of social presence [97, 120] - which as per Deutsch and Gerard [42] is a straightforward determinant of the magnitude of normative influence generated in social groups. For instance, researchers have compared effects of different computer-mediate communication channels on conformity behaviour (e.g. Laporte, Nimwegen, and Uyttendaele [97] compared conformity in groups communicating via textual chat vs. live video stream), and the influence of different types of group members on conformity behaviour (e.g. Midden, Ham, and Baten [120] compared influence of humans vs. virtual agents in eliciting conformity in CMC groups). Similarly, Asch's paradigm has been used in physical groups where the ability to elicit normative conformity is compared between human vs. humanoid robot confederates [66, 172]. Thus, these findings indicate that for experimental studies that investigate effects of social presence or require participants to perceive a higher level of social presence (or a stronger sense of being connected with others in the group), Asch's paradigm is preferred. However, when the focus is on other contextual and personal conformity determinants, Crutchfield's paradigm provides more flexibility and control in how the "group responses" are presented to the participants.

\subsubsection{Determinants of Conformity}

Table 2.1 shows which contextual and personal determinants were investigated in each examined research article. Based on how the literature categorise conformity determinants (as described in Section 2.1.4), we classify Group, Task, and Social Presence as contextual determinants, and Gender, Age, Self-confidence, and Personality as personal determinants of conformity. We observe that $91 \%$ (100 out of 110) of the studies included in the review investigate at least one of these determinants. The remaining 11 studies report a manifestation of social conformity in physical or CMC-based groups, with or without a specific conformity rate - and hence, were retained in our analysis.

In Table 2.2 we present a summary of how often each determinants was investigated in physical and CMC-based groups, and the experimental paradigms used. Furthermore, we note that the $N$ values of each determinant in Table 2.2 indicate the absolute number and the percentage of research articles that have investigated the corresponding determinant out of the 110 research articles considered. However, as most of the studies have investigated more than one determinant (as shown in Table 2.1), the sum of $N$ values goes beyond the total sample size of 110 research papers. In the following sections, we describe our findings with regard to contextual and personal conformity determinants in detail. 
Table 2.2: The distribution of conformity studies based on the determinants they investigate.

\begin{tabular}{lcccccccc}
\hline Determinants & $\mathbf{N}$ & \multicolumn{3}{c}{ Group Setting } & \multicolumn{5}{c}{ Experimental Paradigm } \\
\hline & & Physical & CMC & Both & Asch & Crutchfield & Both & Other \\
\hline Contextual & $\mathbf{7 8}(\mathbf{7 1 \%})$ & $\mathbf{4 9}$ & $\mathbf{2 6}$ & $\mathbf{3}$ & $\mathbf{2 2}$ & $\mathbf{5 1}$ & $\mathbf{3}$ & $\mathbf{2}$ \\
$\quad$ Group & $29(26 \%)$ & 24 & 5 & - & 9 & 20 & - & - \\
Task & & & & & & & & \\
- Type & $22(20 \%)$ & 15 & 7 & - & 2 & 20 & - & - \\
- Objectivity & $8(7 \%)$ & 5 & 3 & - & 1 & 7 & - & - \\
- Difficulty & $16(15 \%)$ & 9 & 7 & - & 3 & 12 & 1 & - \\
Social Presence & $40(36 \%)$ & 16 & 21 & 3 & 16 & 19 & 3 & 2 \\
& & & & & & & & \\
Personal & $\mathbf{6 4}(\mathbf{5 8 \% )}$ & $\mathbf{5 1}$ & $\mathbf{1 2}$ & $\mathbf{1}$ & $\mathbf{1 9}$ & $\mathbf{4 4}$ & - & $\mathbf{1}$ \\
Gender & $38(35 \%)$ & 28 & 8 & 2 & 9 & 28 & - & 1 \\
Age & $12(11 \%)$ & 11 & 1 & - & 3 & 9 & - & - \\
Self-confidence & $13(12 \%)$ & 10 & 3 & - & 6 & 7 & - & - \\
Personality & $28(25 \%)$ & 23 & 5 & - & 7 & 21 & - & - \\
\hline
\end{tabular}

\subsection{Contextual Determinants of Social Conformity}

A significant proportion of early conformity studies in physical groups focused on understanding conformity in terms of its contextual determinants [11, 12, 42, 82]. These studies identify the composition (unanimity and magnitude) of the group that exerts social pressure, and the nature of the experimental task as important contextual determinants of conformity. However, how groups are formed and represented, and how tasks are delivered and completed in CMC-based groups, considerably differ to what has been seen on physical groups [118]. Furthermore, certain factors such as social presence - that are critical to how social conformity influences are perceived, but are implicit in face-to-face settings [118] - require special attention in CMC groups due to how differently they manifest in these settings.

$71 \%$ (78 out of 110) of the research papers analysed have investigated at least one contextual determinant. The breakdown of these papers across these determinants, group settings and the experimental paradigm used is shown in Table 2.2. In general, 49 articles refer to conformity studies that investigate contextual determinants in physical settings, and only 26 articles in CMC-based settings. Another 3 articles report experimental procedures that employ both physical and CMC groups. Moreover, in terms of the experimental paradigms used, we observe that Crutchfield's paradigm has been used more frequently $(n=51)$, than the Asch's paradigm $(n=22)$. The remaining 5 papers have used either both paradigms $(n=3)$, or used no simulations $(n=2)$. The following sections describe how each of these contextual determinants have been investigated in physical and CMC groups, and outline their effects on social conformity behaviour. 


\subsubsection{Majority and Minority Group Size}

Social conformity is a unique form of social influence that arises in group settings when an individual finds their personal opinion contradicting the popular opinion of the group. The popular opinion is often referred to as the "majority's opinion" in conformity literature, where the "majority" refers to the largest proportion of group members that supports a single opinion, while the "majority group size" refers to the number of group members that constitutes the group's majority. Moreover, in the presence of a group majority, there is always a "minority" - which is often where the participants find themselves in, during a conformity experiment. The "minority group size" refers to the number of group members that form the minority. When the majority is unanimous (i.e. when all group members other than the participant supports one opinion) the participant is in a minority of one, whereas when challenged by a non-unanimous majority, the participant in the minority may be accompanied by other group members.

The effect of majority group size on conformity behaviour was the first determinant to be investigated in conformity research [11]. We emphasise that only studies that compare effects of different majority and minority group sizes within a single experimental setup are described in this section. On that note, in the sample considered, 29 research articles (26\% of the total sample) have investigated the effect of majority and minority group sizes on conformity, in either physical $(n=24)$ or CMC-based group settings $(n=5)$. Moreover, depending on which experimental paradigm was used - Asch's $(n=9)$ or Crutchfield's $(\mathrm{n}=20)$ - the majority and minority group sizes were manipulated differently. We have described in detail how these two paradigms control the majority (and minority) group responses across both physical and CMC-based groups in Section 2.1.3.

In general, conformity experiments test for effects of majority group size by manipulating the number of supposed peer responses challenging a participant's judgement. For this purpose, Asch's paradigm uses a predetermined number of (physical or virtual) confederates who announce their responses (as per experimenter's instructions) before the naive participant, to expose the participant to a specific number of controlled responses (or the majority's opinion) [11]. In other words, the number of confederates inserted to the group determined the majority group size (assuming that confederates are instructed to remain unanimous in their responses to critical trials). Moreover, a few studies have analysed effects of non-unanimous majorities using Asch's paradigm, either by instructing one or more confederates to respond differently to the majority [11, 12], or by including two naive participants (in addition to the confederates) in the same group session, expecting the first participant to answer correctly to the experimental task and break the unanimity of an incorrect majority of confederates [11, 12]. Furthermore, majority group sizes in the range of 1-16 - in the presence of one or no dissenters have been tested using Asch's paradigm in physical groups. However, prior work does not report using Asch's paradigm to test group size effects in CMC groups. Hence, in Chapter 7 we present a study where the effects of majorities of three or four peers, in the presence of one or no dissenters, has been investigated. In this experiment the perceived level of social presence within the group was also manipulated through the presence or the absence of a real-time discussion between users - in addition to manipulating the majority-minority group composition - for which confederates were necessary. 
Conversely, Crutchfield's paradigm uses simulations to indicate the number of majority and minority responses (i.e. the majority and minority group sizes) to a group of independent naive participants simultaneously - without using confederates. In physical conformity experiments where the Crutchfield's original apparatus (with or without modifications) was used, the largest possible majority group size that can be simulated was determined by the number of naive participants undertaking the experiment at the same time. For instance, Mouton, Blake, and Olmstead [123] exposed five naive participants in adjoining soundproof booths, to four simulated responses supposedly coming from the others taking part in the experiment with them. This enabled authors to expose naive participants to a unanimous majority of four, as well as to four responses equally split across two response options, to test effects of both unanimous and nonunanimous majorities on conformity. Similarly, several other studies have used the original Crutchfield's apparatus, to investigate effects of majority group sizes between $1-4$, and minority group sizes between $0-2[4,7,64,141,142]$. However, when other types of simulations were used to indicate majority group size - i.e., number of previous signatures on a petition [164] - effects of larger majority group sizes between 0-12 were investigated.

All except one article considered in the review that investigated group size effects on conformity in CMC groups, have used Crutchfield's paradigm. We note that some of these studies have opted for mimicking the original Crutchfield's apparatus using computer software, where fabricated peer responses were sequentially displayed using supposed peers names or avatars, to test effects of majority group sizes between 1-6 and minority group sizes between $0-3[106,159]$. Therefore, this approach where individual peer responses are displayed one after the other, seems better suited for testing effects of comparatively smaller majority-minority group sizes. However, smaller group sizes can restrict the different combinations of majority-minority group compositions that can be investigated (e.g. with 4 simulated peers, majority group size could only be either 3 or 4). Conversely, another set of studies have used fabricated bar charts [139], or simply indicated the aggregated number of peer votes supporting each response option [188], which allowed them to test majority-minority group size effects in larger groups (e.g. Zhu, Huberman, and Luon [188] showed peer votes in the range of 150-200 across the majority and minority groups, to indicate larger group sizes), as well as in numerous majority-minority group compositions.

\subsubsection{Effects of the Majority and Minority Group Sizes on Conformity Behaviour}

The effect of the "majority" has been investigated across two main aspects - its size and unanimity. At first, the focus was on understanding effects of unanimous majorities of different sizes, against one naive participant or a minority of one $[11,12,141,142$, 164]. Researchers then investigated how disrupting the unanimity of the majority by inserting dissenters who challenge the majority's response impacts conformity in naive participants $[4,7,72,122,123]$. To ensure a fair comparison, we only analysed studies where the unanimity and/or the size of the majority varied across experimental conditions within the same experiment, and does not compare group sizes between different studies. 


\subsubsection{Conformity in Unanimous Majorities}

Asch [11] was the first researcher to investigate the effects of unanimous majorities of different sizes on conformity in physical groups. Asch conducted a between-participants study, where naive participants completed the seminal line judgement task (a visual perception task, objective in nature) facing 1, 2, 3, 4, 8, or 16 confederates. Corresponding conformity rates of each of the six experimental conditions were then used to determine the incremental impact of each additional member of the majority on pressure to conform. Based on this analysis, Asch [11] observed that conformity rates gradually increased until the 3rd member of the majority, and that larger majorities did not produce effects greater than a majority of three. Subsequent studies further confirmed a similar curvilinear relationship between majority group size and conformity, such that a majority of three was deemed sufficient for the full impact of the majority to be felt [12, 141, 142]. Furthermore, Stang [164] also observed a curvilinear relationship between majority group size and conformity behaviour in a task of subjective nature, where the probability of a user signing a petition increased when they saw four other signatures compared to seeing no signatures, which subsequently levelled off in the presence of eight and twelve signatures. Thus, increasing the size of a unanimous majority after a certain point merely confirms that the majority constitutes a representative sample, and therefore creates no additional pressure to conform in physical groups [12].

However, previous work has also shown that effects of unanimous majorities on conformity can sometimes be dependent on other variables [94, 117]. For instance, Insko et al. [82] compared conformity behaviour in participants whose public or private responses were challenged by unanimous majorities of three or six, in tasks where a relationship between two colours could be objectively determined (there exists a correct answer) or not. The researchers note that while larger majorities triggered more conformity, the group size effect was more evident in determined (objective) than in undetermined (subjective) tasks, and in public (i.e. responses visible to other group members) than in private responses (i.e. responses not visible to other group members). They describe that larger group sizes enhance users' susceptibility to informational (need to be right) and normative (need to be liked) influences, particularly in the presence of objective tasks and public responses respectively.

Furthermore, recent CMC-based conformity studies also compare conformity behaviour among small vs. large unanimous majorities. In an objective, visual perceptual task (indicating similarity between two dot patterns), Campbell and Fairey [28] note that participants are more likely to conform when exposed to four computer-simulated responses of supposed peers, than when they were exposed to only two other computersimulated peer responses. Lee and Nass [106] report similar behaviour in a subjective, choice-dilemma task where participants were more willing to conform when challenged by four "peer" responses than one. They further explained that this majority effect was only visible when participants were informed that their peers are human (even when it was not the case in reality), and not when participants presumed their partners to be computer agents. This led them to conclude that normative pressures eliciting conformity in subjective experimental tasks, exist in human-human interactions but not in human-agent interactions. Therefore, findings of prior work with regard to majority 
group size in CMC settings suggest that while larger unanimous majorities can trigger more conformity than smaller majorities in general, this effect can manifest differently in subjective vs. objective tasks, and based on the level of social presence perceived by users. Hence, in Chapters 4, 6 and 7 we investigate how effects of majority group size on conformity in CMC groups are moderated by effects of subjective vs. objective nature of the experimental task as well as the level of social presence perceived by users.

\subsubsection{Conformity in Non-unanimous Majorities}

An experimental setup where a unanimous majority challenges a minority of one (the naive subject) does not account for the fact that our personal judgements (especially when correct) can be supported by others who share the same perspective. Therefore, the above observations regarding effects of unanimous majorities on conformity may not be applicable to realistic group settings. In response to this argument, Asch [11] repeated the line judgement task where one confederate within the group was instructed to always support the answer of the naive subject, to break majority's unanimity. Asch observed that disrupting the majority's unanimity significantly diminished its effect on conformity rate, which dropped drastically to $5.5 \%$ from the previously observed $33.3 \%$. Furthermore, to clarify if it was the dissenting behaviour or the accuracy of the dissenter's response that discouraged conforming behaviour, Asch [12] compared participants' conformity in the presence of a dissenter who always supported their correct answer, a dissenter who made moderately erroneous judgements and a dissenter who made extreme errors. The results showed that while pressure to conform was reduced in all three scenarios, it was lowest in the presence of an extremely erroneous dissenter. These findings indicate that while the act of dissenting itself increases independence in naive participants, extremely erroneous dissenters create the most impact.

Subsequently, Allen and Levine [4] extended the investigation of the effects of nonunanimous majorities beyond visual perceptual tasks. In a quiz containing visual, opinion and factual items, the effects of supportive (agrees with the subject) and extremely erroneous dissenters were investigated. Their findings showed that while a supportive dissenter can reduce conformity across all three task types, extremely erroneous dissenters only reduce conformity in visual and factual tasks (that are objective in nature). As such, Asch's claim about erroneous dissenters reducing pressure to conform remains valid only for tasks of objective nature. Allen and Levine [4] further explained that lack of group consensus in objective tasks can insinuate that the majority may be wrong (diminishing informational influence), whereas in subjective tasks the group majority remains a source of social comparison - regardless of its unanimity (normative influences remain unchanged).

Furthermore, studies that investigate effects of non-unanimous majorities on conformity behaviour in CMC groups, present slightly different insights to what has been observed in physical groups. For example, Walther et al. [176] conducted a betweenparticipants experiment where the effect of two dissenters was compared against a majority of three and a majority of eight in a memory judgement task. Their findings indicated that the dissenting behaviour only resulted in a meaningful reduction in conformity against a majority of three, and not against a majority of eight. As such, 
the dissenter effect seems to be restricted by the size of the opposing majority in CMC groups - which requires further investigation. Therefore, in Chapters 4, 6, 7 and 8 we examine effects of different majority-minority group compositions, to quantify for effects of both the majority group size as well as the minority group size on user conformity behaviour. Furthermore, in Chapter 4 we also investigate potential effects of having multiple minorities opposing the majority's judgement, to further understand the dissenter effect.

\subsubsection{Experimental Task}

Conformity behaviour has been observed across a wide variety of task types such as - perceptual (e.g., visual judgement and auditory perception tasks [11, 167]), logical (e.g. completing a mathematical series [187]), factual (e.g. general knowledge [42]), attitudinal (e.g. personal attitude on a societal topic [39]) and preference-based (e.g. choosing the preferred line drawing out of two options [39])) tasks. Furthermore, these experimental tasks can also be grouped into two distinct categories based on their objective or subjective nature - i.e. their task objectivity. For instance, perceptual, logical and factual tasks often have a single correct answer and thus, are objective in nature. On the other hand, attitudinal and preference-based tasks are subjective in nature in that they do not have one "correct" response. Moreover, the experimental tasks can also vary based on task difficulty. This term describes the perceived difficulty of the task as well as the ambiguity associated with it (e.g. adding two numbers together vs. completing an insolvable mathematical series).

Hence, we focused on the effects of the experimental task on conformity across three main aspects - task type, task objectivity and task difficulty. We note that a significant $32 \%$ of the studies $(\mathrm{n}=35)$ considered in this survey analyse the effects of at least one of these aspects - in either physical $(n=22)$ or CMC-based $(n=13)$ groups - as shown in Table 2.2. We note that only studies that compare conformity behaviour across different task types $(n=22)$, between subjective or objective nature of tasks $(n=8)$, or different levels of task difficulty $(\mathrm{n}=16)$ within one experimental setup, have been considered to identify potential variations in conformity. In the following sections we explain how these three aspects were investigated in prior work, before discussing their impact on social conformity in Section 2.4.2.4.

\subsubsection{Task Type}

Conformity was first tested in perceptual tasks - i.e. the line judgement task [11] and counting metronome clicks [123] - where participants have to rely of their (visual or auditory) senses to make a judgement. Using perceptual experimental tasks where no additional knowledge was required to select the correct answer allowed researchers to explain conformity behaviour in terms of normative social influences. For example, as Asch [11] observed that the likelihood of participants making an error when completing the line judgement task without peer feedback was less than 1\%, it is highly likely that participants conformed to incorrect majorities in group settings because of their need 
to fit in with the majority's decision (normative influences), and not because of their inability to determine the correct answer.

Later on, researchers were interested in how conformity behaviour would generalise across different types of experimental tasks. Crutchfield [39] tested the impact of logical, factual, attitudinal and preference-based tasks - in addition to perceptual tasks - to examine the generalisability of Asch's observations to other task types. Participants were asked to complete number series (logical), judge whether pairs of words are synonyms or antonyms (factual), estimate others' opinions on attitudinal topics (e.g. "I doubt whether I would make a good leader") and express personal preference on line drawings under group settings. Motivated by Crutchfield's attempts, many others have subsequently investigated effects of task type on conformity behaviour - across perceptual (visual and auditory), logical, factual, attitudinal and preference-based tasks. We provide several popular examples for each of these task types in Table 2.3.

Table 2.3: Examples of different task types used in conformity studies.

\begin{tabular}{ll}
\hline Task Type & Examples \\
\hline Perceptual (visual) & $\begin{array}{l}\text { Select the larger out of two clusters of dots [37], and two shapes [157] } \\
\text { Select the longest line in a set of lines [51] } \\
\text { Count the number of dots on a slide [122] } \\
\text { Count the number of metronome clicks on a recording [20, 123] } \\
\text { Select the recording with the longer tone [144] } \\
\text { Complete a number series [39] } \\
\text { Cogical }\end{array}$ \\
$\begin{array}{l}\text { Complete a simple arithmetic task [20, 139] } \\
\text { Complete a general knowledge quiz [97] }\end{array}$ \\
Complete a knowledge inventory [51] \\
Attitudinal & $\begin{array}{l}\text { Indicate decision in a hypothetical choice-dilemma scenario [106] } \\
\text { Indicate attitude on moral and political questions [97] } \\
\text { Indicate preference between two pictures [188] }\end{array}$ \\
Preference-based & Indicate preference on a political debate [111]
\end{tabular}

We do not observe significant differences between types of experimental task used in CMC and physical conformity studies. In terms of perceptual tasks, we note that the line judgement task has been replicated quite often in CMC-based conformity studies (e.g., [97, 120]). Similarly, multiple-choice question (MCQ) quizzes including questions of logical (completing number series or solving arithmetic sums), factual (testing vocabulary and general knowledge) and attitudinal (political and moral opinions on topics of societal interest) nature have been used in CMC groups as previously seen in physical groups [97, 139]. Furthermore, conformity behaviour has also been tested in preference-based tasks in CMC groups. For example, Zhu, Huberman, and Luon [188] investigated conformity in how participants choose between two photographs that could be used to advertise baby products online, whereas Maruyama et al. [111] tested conformity in how participants vote for two political parties after being exposed to an online debate on Twitter. 


\subsubsection{Task Objectivity}

Task objectivity varies based on the task type. Perceptual, logical and factual tasks often have a "correct" response that can be objectively determined. Conversely, attitudinal and preference-based tasks are subjective in nature, where there could be a "popular" response, but no single "correct" response. We note that conformity studies that use subjective experimental tasks often use control groups to identify the "popular" attitudes, opinions and preferences of the target community. For instance, Blake, Helson, and Mouton [20] used a control group to determine the popular opinions on a set of attitudinal statements related to war and peace (e.g. "There is no progress without war", "Only cowards oppose war"). Similarly, Allen and Levine [4] tested conformity behaviour in opinion items (e.g. "Most young people get too much education") by simulating the responses of the "majority" to be in the 95th percentile of responses given by control participants (in other words, only $5 \%$ of control participants supported the simulated majority's response).

\subsubsection{Task Difficulty}

Task difficulty (or task ambiguity) determines the effort that is required to identify the "correct" answer in objective experimental tasks. This notion was first tested in 1955 by Deutsch and Gerard [42] with regard to visual perceptual tasks. Participants completed two variations of the line judgement task, where they were required to make a judgement when the stimulus lines were visually present (visual) vs. three seconds after the lines were removed (memory). The researchers argued that the absence of visual proof of the "correct" answer would make participants more perceptive to the majority's judgement. Similarly, in auditory perceptual tasks such as counting the number of metronome clicks on a tape recording, task difficulty has been manipulated by controlling for the click rate per minute $[20,114]$. Trials with a higher click rate (more clicks played per minute) were considered as more difficult than others.

Alternatively, task difficulty can be more objectively determined based on how a control group of participants complete the experimental tasks in private settings [31, 157]. For example, Blake, Helson, and Mouton [20] analysed responses of a control group $(\mathrm{n}=50)$ to logical (solving arithmetic questions) to categorise questions with $>75 \%$ of correct answers as "easy", 25-75\% correct answers as "moderate" and less than $<25 \%$ of correct answers as "difficult". They ensured that control group participants were recruited from the same population as the experimental participants, to ensure the validity of this approach (i.e. two samples of college students with similar education levels). The same approach has been used to identify task difficulty in factual questions by Coleman, Blake, and Mouton [31].

The processes of controlling for task difficulty in CMC groups are quite similar to what has been observed in physical settings. For instance, Midden, Ham, and Baten [120] compared between low vs high task difficulty in a replication of Asch's line judgement task in a CMC setting, by either displaying the relevant stimulus lines throughout each trial (low difficulty) or by visually removing the lines after only displaying them for 0.35s (high difficulty). Similarly, the control group approach was used by Rosander and Eriksson [139] to determine the objective task difficulty in factual and logical questions. 
Based on the percentage of correct answers for each question, objective task difficulty was determined as "easy" (90-100\% correct answers), "moderate" (80-89\% correct answers) or "difficult" ( $<80 \%$ correct answers). Furthermore, this work also introduces the notion of "subjective" task difficulty, where the perceived difficulty in answering each questions was indicated by experimental participants on a scale of $1-5$, which was later used to categorise tasks into low $(1,2)$ vs. high (3-5) subjective task difficulty groups.

\subsubsection{Effects of the Experimental Task on Conformity Behaviour}

Effects of task type, objectivity, and difficulty on conformity behaviour are often described in tandem within the conformity literature. Crutchfield's study [39] was one of the first to explore how conformity manifest across different task types. The Crutchfield's apparatus was used to ensure that the level of social pressure perceived by a group of five naive participants was kept constant as they completed tasks of perceptual, logical, attitudinal, opinion-based and preference-based nature. They observed conformity rates ranged from $2 \%-46 \%$ across the five task types, such that preference-based tasks yielded the lowest conformity $(2 \%)$, while perceptual tasks resulted in the highest conformity (46\%). The researchers concluded that the conformity behaviour observed by Asch in his line judgement experiment [11] can manifest in other task types - but with different magnitudes.

Blake, Helson, and Mouton [20] provided more insights on effects of task type on conformity, using the Crutchfield's apparatus in a group size of five (same as in [39]). They observed conformity rates of $30 \%, 27 \%$ and $41 \%$ in auditory perceptual (counting metronome clicks), logical (solving arithmetic problems) and attitudinal tasks respectively. In other words, social pressure is much more effective in triggering conformity behaviour in attitudinal tasks with no "correct" answer, than in perceptual and logical tasks with an objectively identifiable "correct" answer. They rationalised that the need to select the "correct" answer in objective tasks may have outweighed the perceived social pressure towards conforming to an obviously incorrect majority, leading to lower conformity in perceptual and logical tasks. Conversely, in subjective content where there is no single "correct" answer, people are eager to conform to responses accepted by the majority to avoid undesirable situations (normative influence). Therefore, it is clear that while conformity manifests in both subjective and objective tasks in physical groups, its effect is stronger in subjective content because of higher susceptibility to normative influences.

Effects of task type on conformity have also been investigated in CMC-based groups. For instance, Laporte, Nimwegen, and Uyttendaele [97] deployed an online quiz where one naive participant completed two types of objective tasks (i.e., Asch's line judgement task and a set of factual general knowledge questions) and two types of subjective tasks (i.e., attitudinal and preference-based questions), in the presence of five virtual confederates. Their results indicated no conformity in the Asch's line judgement task and only $15 \%$ conformity in the factual tasks, whereas comparatively higher levels of conformity were observed in attitudinal (30\%) and preference-based (20\%) tasks. While these results are not directly comparable with what has been observed in physical groups, they indicate that conformity can manifest across a variety of task types in CMC groups, similar to physical groups. 
While initial results from Laporte et al's study showed higher conformity in subjective tasks than in objective tasks, these observations were challenged by more recent studies. For example, Hertz and Wiese [78] reported significantly higher user conformity (46.5\%) in objective, analytical tasks (i.e. completing arithmetical sums) than in social tasks (i.e. judging the emotion indicated by a photograph), that are subjective in nature (with a conformity rate of 35.6\%). Moreover, Perfumi et al. [134] compared conformity between the seminal line judgement task - which is argued to elicit normative influences (see 2.4.2.1) - and a vocabulary-based word matching activity (designed to elicit informational influences due to its factual nature), in a anonymous online group setting. They reported that conformity was almost non-existent $(1.4 \%)$ in the line judgement task, whereas almost $30 \%$ conformity was observed in the vocabulary task. They explained that 'deindividuation' effects (losing awareness of self in a group) caused by anonymity and physical isolation in CMC groups can act as an inhibitory factor for normative influences, which resulted in non-existent conformity in the line judgement task. Conversely, the effect on informational influences is lower because when the objective is to gather as much information as possible from the group to arrive at the correct answer, even anonymous users can be considered a reliable source.

Therefore, to resolve these contradictory findings with regard to effects of task objectivity on conformity and further understand how task objectivity determines users' susceptibility to informational and normative influences that trigger conformity, in Chapters 4 and 7 we investigate CMC-based user conformity in both subjective and objective experimental tasks. Chapter 7 in particular, examines effects of task objectivity on user conformity in high vs. low levels of perceived online social presence.

Regarding the effects of task difficulty on conformity behaviour, these have been consistent across both physical and CMC-based conformity studies - higher task difficulty results in higher conformity. With regard to visual perceptual tasks, Deutsch and Gerard [42] state that by removing the visual stimulus, perceived task difficulty can be effectively increased in the line judgement task in physical groups. Similar results have also been reported by Midden, Ham, and Baten [120] in a CMC group by reducing the presentation time of the stimulus. In both cases, conformity was heightened in the presence of tasks with higher perceived difficulty. Deutsch and Gerard [42] explained that when participants are exposed to tasks with higher difficulty, where the "correct" answer is unclear, they were more likely to conform to the majority for informational reasons (i.e. majority becomes a source of information).

Regarding auditory perceptual tasks, previous work has shown that where participants were required to count the number of metronome clicks in a tape recording - where the click rate was manipulated to control for task difficulty - Sistrunk and McDavid [157] observed that task difficulty tends to result in higher conformity. They described that the "susceptibility to group pressures on individual judgements is greater in situations in which objective determinants of the judgement are indefinite or subjectively ambiguous than in situations in which evidence for objective determinants of the judgement is readily and amply available". In other words, increasing the click rate resulted in enhancing participants' susceptibility to informational influences, because the "correct" answer was not clear or easy to determine. These findings resonate with what was initially observed by Deutsch and Gerard [42] in visual perceptual tasks. However, Blake, Helson, and 
Mouton [20] did not observe significant differences in conformity between low vs. high task difficulty in a similar auditory perceptual task. On one had, Sistrunk and McDavid [157] identified "easy", "difficult" and "insoluble" tasks based on the performance of control participants, before deploying the main experiment. On the other hand, Blake, Helson, and Mouton [20] simply used low and high click rates to control for low vs. high task difficulty, which they later described as a possible reason why expected effects of task difficulty did not manifest. The effects of task difficulty on conformity in auditory perceptual tasks is yet to be investigated in a CMC setting.

Higher task difficulty can also heighten conformity in factual and logical tasks in both physical and CMC groups. For example, Blake, Helson, and Mouton [20] and Coleman, Blake, and Mouton [31] show that by using the performance of a set of control participants to categorise arithmetic and factual tasks as "easy", "moderate" and "difficult", prior observations with regard to task difficulty and conformity in perceptual tasks can be extended to tasks of logical and factual nature, in physical groups. Similar observations have been found by Rosander and Eriksson [139] in a CMC group, where conformity was seen to significantly increase with higher objective task difficulty (determined based on the performance of control group participants) as well as with higher subjective task difficulty (participants' perceived difficulty in answering the questions).

Moreover, task difficulty has also been investigated in physical groups in connection to "task importance" - which determines incentives for accuracy. In other words, high task importance can encourage people to seek the "correct" answer, whereas low task importance can lead to people being more comfortable with their mistakes. Baron, Vandello, and Brunsman [16] provide a good example for manipulating task importance in a visual perceptual task, where participants were shown an individual whom they had to later identify in a line-up. In high task importance condition participants were told that accuracy of their responses is critical and that their performance in the task will later be used to distinguish between good and bad eye witnesses by the police. Conversely, in the low task importance condition, participants were told that the experiment is a pilot study designed to develop materials for eyewitness accuracy. Furthermore, the researchers controlled for task difficulty by manipulating the presentation time of the visual stimulus. They note that when participants perceived the experimental task to be of low importance, low and high task difficulty did not significantly differ in conformity behaviour (33\% and 35\% conformity respectively). However, when the importance of the task was heightened, high task difficulty encouraged more conformity (51\%), when compared to low task difficulty (16\%). These findings establish the relationship between task difficulty and informational influences. Increasing task difficulty will heighten conformity only when susceptibility to informational influences is enhanced - which can be experimentally induced through high task importance (increasing the need to obtain the correct answer). Alternatively, in lower task importance the previously seen effects of task difficulty disappear, as a result of lower susceptibility to informational influences. However, these findings are yet to be extended into CMC groups. 


\subsubsection{Social Presence}

The term "social presence" is described as the "degree of salience of another person in computer-mediated interactions" [156]. Moreover, as per Short, Williams, and Christie [156] the level of perceived social presence will also determine the "consequent salience of the interpersonal relationship" among CMC group members. Hence, while less important in physical settings where group members are face-to-face, in CMC groups it is a critical determinant of the perceived level of social influence. Furthermore, Tu and McIsaac [170] note that social presence is multi-faceted and thus manifests across many dimensions each with its own set of variables. The researchers further describe that the presence of social context cues (e.g. user characteristics), the level of interactivity (e.g. communication strategies) and the level of privacy implemented (e.g. response visibility: user responses are publicly visible to others in the group or private) are common dimensions of social presence in CMC platforms.

Social presence is an important and often investigated contextual determinant of conformity. We found a total of 40 research articles (36\% of the total sample) investigating effects of social presence across four aspects - level of social context cues present (e.g., anonymous vs. identifiable self and/or peers, user representations used in CMC), level of interactivity allowed between group members (e.g., means of interaction allowed before, during or after the experiment with peers), response visibility (e.g. public vs. private responses of self and/or peers), and humanness of supposed "peers" (the quality of being human or not; e.g., human peers vs. robots vs. virtual agents). While the latter is not a dimension of social presence as per Tu and McIsaac [170], the humanness of supposed peers directly impacts "the degree to which people are perceived as real and present" - which determines perceived social presence [70]. We further note that this notion is different to testing effects of high or low anthropomorphic user representations because from the users' perspective CMC-based user representations were supposedly representing human partners. In other words, users were not aware that their peers are computer-simulated or non-human. Hence, we discuss effects of peer humanness on perceived social presence and conformity in this review for completeness.

Furthermore, as seen in Table 2.2 - out of the considered conformity determinants - social presence is the only determinant that has been more frequently investigated in CMC groups $(n=21)$ than in physical settings $(n=16)$. We also note that three research articles included in this survey have investigated effects of social presence in both physical and CMC contexts $[29,68,69]$. On that note, we highlight that we only considered studies that have tested how conformity manifest in different levels of social presence within the same experimental setup. Next, we describe how social presence was manipulated in physical and CMC-based conformity studies in terms of the social context cues used, level of interactivity allowed, response visibility provided, and humanness of supposed peers.

\subsubsection{Social Context Cues}

In physical groups, the level of social context cues was manipulated in terms of the anonymous or identifiable nature of participants. After Asch's observations where 
participants conformed to a clearly incorrect yet unanimous majority in a public setting, it was argued that participants may have conformed because their personal identity was revealed to others [123]. Gerard [60] further described that in Asch's setup, participants' judgements are not only in response to the obvious discrepancy in the majority's argument but also to what they believe are the expectations of the group as to what their response should be (normative influence). Thus, researchers then investigated the effects of a clearly incorrect majority when participants were identifiable, and when they were not. For instance, Deutsch and Gerard [42] and Gerard [60] compared conformity in the line judgement task across Asch's paradigm - where participants were expected to respond publicly in the presence of their peers (face-to-face condition) - and Crutchfield's paradigm - where participants could indicate their judgements through Crutchfield's apparatus without exposing themselves (anonymous condition). Alternatively, Mouton, Blake, and Olmstead [123] asked half of their participants (and supposed peers) to indicate their name before giving out their judgements using the Crutchfield's apparatus, whereas the other half did not disclose any identifiable information.

There has also been similar CMC-based conformity studies. For example, Perfumi et al. [134] compared conformity behaviour between groups of participants who remained anonymous (identified by a number representing their response order) and groups where each participant was identifiable through their first and last names. Furthermore, literature that investigates effects of social context cues on conformity in CMC groups have also looked into effects of CMC-based user representations. They have compared conformity behaviour in groups that shared a common avatar and groups that used different avatars to represent each member $[89,103]$, and effects of anthropomorphic and non-anthropomorphic user representations on conformity behaviour (e.g. Lee and Nass [106] compared conformity against peers represented by text boxes, stick figures and animated characters).

\subsubsection{Interactivity}

The level of interactivity encouraged between group members can also determine to what extent users perceive social presence and feel pressured to conform. This has been investigated in a physical setting by Allen and Levine [5], where the impact of prior contact between the participant and a group member was manipulated by allowing participants to either interact (for a period of 5-minutes) with a confederate before the experiment, or not. Conversely in CMC settings, the level of interactivity has been controlled by the richness of CMC channels used for group interactions (e.g. Laporte, Nimwegen, and Uyttendaele [97] compared conformity in participants who either communicated their responses with others through a text-based group chat, or using live-video streams), or by limiting how users are allowed to interact with their group members (e.g. Maruyama et al. [111] compared conformity in participants who either actively interacted with tweets posted by others in the community on a topic of societal interest, or simply observed others' tweets without responding to them). 


\subsubsection{Response Visibility}

Several studies have investigated how the public or private nature of user responses (response visibility) can determine social conformity. For example, Insko et al. [82] and Abrams et al. [1] analysed differences in conformity behaviour when participants in physical groups were asked to publicly announce their judgements (public responses) and when they were asked to write their judgements on piece of paper with no identifiable information (private responses). Moreover, Eagly, Wood, and Fishbaugh [48] investigated how participants would conform when they were told their responses would be used by group members to rate each member vs. when they perceived their responses to be private. Conversely, in CMC groups, the effects of response visibility has been tested by comparing participants' public responses and private responses to the same task. For instance, Lee and Nass [106] asked participants to answer a series of social dilemma questions both in the presence of a simulated group and in private where they answered the same question set on a piece of paper.

\subsubsection{Humanness of Peers}

More recent conformity studies - in both physical and CMC groups - test conformity behaviour against non-human peers. For instance, studies by Brandstetter et al. [24], Shiomi and Hagita [155], and Vollmer et al. [172] replicate Asch's line judgement task in physical groups, where participants responses are challenged by robots instead of human peers. Brandstetter et al. [24] in particular compared user conformity behaviour when personal judgements to perceptual and factual tasks are either challenged by human or robotic peers. Similar work was conducted in CMC groups by Midden, Ham, and Baten [120], where user conformity was analysed when users' judgements to the line judgement task were challenged by virtual agents and computer programs - in addition to human peers. Furthermore, other studies have also compared the influence of human vs. non-human peers on user conformity behaviour in social and analytical tasks [78], to determine whether these effects vary across different task types.

\subsubsection{Effects of Social Presence on Conformity Behaviour}

Social context cues: The effects of social context cues has been tested by comparing conformity between face-to-face and anonymous physical group arrangements. Seminal studies that tested this notion in the line judgement task observed less social influenced errors (or conformity behaviour) in the anonymous condition than in the face-to-face condition $[42,60]$. This behaviour was explained by Gerard [60] as enhanced susceptibility to normative social influences in face-to-face groups than when anonymous. In other words, the pressure to "maintain face" within the group is heightened when opposing group members are visible. They further compared the number of participants who made five or more errors in face-to-face $(n=18)$ and anonymous $(n=8)$ groups, to emphasise that conformists tend to conform more in face-to-face settings, as a result of heightened normative influences. Mouton, Blake, and Olmstead [123] showed similar results in an auditory perceptual task, where higher conformity was observed when participants and simulated peers provided their names before responding to each question (and therefore 
were identifiable), in comparison to when group members were anonymous. However, this behaviour was true only for participants who were categorised as "submissive" as per their scores in the A-S Reaction survey [9]. By definition, "submissive" users are more influenced by social context than their counterpart - "ascendants". Mouton, Blake, and Olmstead [123] noticed no significant differences in conformity in anonymous vs. identifiable groups among participants who were categorised as "ascendants". Therefore, effects of social context cues on level of perceived social presence and resulting conformity behaviour should be analysed in relation to various personal differences between participants in future.

Similarly, deindividuation caused by anonymity and physical isolation in CMC groups has been shown to discourage conformity - especially in tasks that appeal to normative influences [134]. However, anonymous CMC groups can also be experimentally primed towards informational or normative behaviours. Spears, Lea, and Lee [162] showed that when anonymous online groups were exposed to either pro-social or efficiency-oriented norms, they behaved as per the experimentally induced norm (displaying either pro-social or information seeking behaviour) in a later social dilemma task. Such prime-oriented behavioural conformity could not be successfully induced in identifiable CMC groups. Thus, existing work is insufficient to fully understand effects of anonymity on conformity in CMC settings.

User representations can also impact how users perceive each other in CMC groups. For instance, Lee [103] found that participants perceived peers as similar to them when they shared the same cartoon avatar, than when they were assigned unique cartoon avatars. They also note that using one avatar to represent all group members can result in de-personalisation - which can diminish perceived social presence among group members. Moreover, authors indicate that in a depersonalised situation, experimentally inducing perceptions of group membership can lead to higher conformity behaviour, whereas inducing perceptions of self-identity can lead to lower conformity behaviour. However, highlighting group/self identity did not impact conformity behaviour when all members were represented using unique avatars. In Chapter 7 we investigate how CMC-based user representations impact conformity behaviour when group or self-identity is not experimentally induced, to better understand how user representations can make users more or less susceptible to other salient norms in CMC groups that can influence their conformity behaviour.

Highly anthropomorphic (or human-like) user representations can also induce stronger perceptions of social presence in CMC groups. For instance, Lee and Nass [106] found that participants assumed peers represented by animated characters to be more intellectual, trustworthy and socially attractive than their counterparts represented using text boxes and stick figures. However, contrary to expectations, participants were seen to show greater conformity to group opinions when they were represented by low anthropomorphic text boxes, than high anthropomorphic animated characters. The researchers later rationalised that visual differences between animated characters may have emphasised intragroup differences that can encourage self-identity, whereas text boxes that lacked visual differences may have indirectly induced a sense of group membership. Thus, the between-participants experimental design of this study (where participants were exposed to only one form of user representation), may not have 
effectively distinguished effects of anthropomorphism from previously discussed effects of salient group/self-identity norms [103].

Interactivity: The level of interactivity allowed between members of physical groups can lead to increased rapport between group members. For instance, Allen and Levine [5] showed that when a naive participant and a confederate are allowed to interact briefly before the experimental task, the participant perceives themselves as a social unit that is differentiated from the rest of the group. As a result, the participant (who is also informed of the position of their "peer" in the group) mimics the behaviour of this particular partner - regardless of whether the partner conform or dissent to the majority's decision. In other words, when the simulated majority (using Crutchfield's apparatus) was unanimous, participants who interacted with a supposed peer (who in reality was a confederate) conformed more than those who had no interaction. Alternatively, when the "peer" dissented to the majority's position (to test effects of non-unanimous majorities), participants who interacted with the said peer conformed less than those who did not.

Interactivity has been controlled in terms of richness of CMC medium and means of interactivity allowed in CMC-based conformity studies. Laporte, Nimwegen, and Uyttendaele [97] found that participants who completed an online survey conformed more to majority's responses when they indicated their answers to supposed peers via live video streams, than when they had to use a text-based group chat for communication. Furthermore, the reported conformity rates in text-based chat and live video conditions for factual (15\% vs. 28\%), opinion-based (30\% vs. 33\%) and preference-based (20\% vs. $24 \%$ ) questions in the survey shows a consistent trend for higher conformity in the live video condition. Moreover, when the public (in the presence of the group) and private (paper-based survey after the experiment) responses of participants were later analysed, authors found an astounding $51 \%$ of changes in the live video conditions in comparison to only $18 \%$ of changes in the text-based chat condition - which indicates considerable levels of public compliance in the former. As public compliance is an indication of susceptibility to normative influences, their results suggest that higher social presence can induce higher susceptibility to normative conformity in CMC groups.

Maruyama et al. [111] investigated effects of interaction in a social watching experiment, where participants were asked to indicate their preferential candidate to the 2012 US election before and after watching the official televised election debate, while simultaneously following election-related posts on Twitter. They found that participants who were instructed to actively engage with the tweets (by posting their own thoughts) were more likely to change their pick for the preferential candidate to align with the majority's judgement on Twitter (33\% conformity) than those who simply observed tweets without interacting with them (7\% conformity). They concluded that the added interaction on Twitter significantly enhanced users' receptiveness to the majority's sentiment, which in turn determined their conformity behaviour.

On that note, we emphasise that the above studies have investigated effects of interactivity in CMC groups primarily focusing on real-time synchronous interactions (e.g. real time text-based chat, live video streams, tweets posted during a particular time frame). Thus, it is unclear if these findings can be extended to asynchronous interactions that are equally or more prevalent in modern online settings (e.g. group forums, social media). 
Response visibility: Participants' responses to an experimental task can either be publicly visible or hidden to other group members. While earlier studies investigated how conformity manifest in public settings (e.g. in Asch's line judgement task all group members announced their answers publicly [11, 12]), researchers then tested how surveillance of participants' responses (and lack there of) can impact their conformity behaviour [1, 47, 48, 82]. These studies in general indicate that when responses are publicly visible to the group, participants in both physical and CMC-based group conform more, than when individual responses are kept private. For example, Eagly and Chrvala [47] found that participants who were informed that they will be required to announce their personal opinions to the group in a later discussion were more likely to conform to the majority's opinion on the topic (which they were exposed to before giving their own opinion), than those who were informed that their personal opinions will not be seen by group members nor will be used in the later discussion. Similarly, in a CMC-based group Lee and Nass [106] observed higher conformity in a social dilemma task when participants indicated their responses in the presence of a simulated group, than when they completed the same task in private (on paper). Deutsch and Gerard [42] explained that public responses can increase susceptibility of users to normative influences, which in turn can increase pressure to conform. Conversely, in private responses the motivation to conform to an incorrect majority solely for normative reasons (to "fit in" or be accepted) is reduced.

However, effects of response visibility on conformity can not be quantified in isolation. For instance, Insko et al. [82] observed that response visibility interacted with majority group size in physical settings, such that public responses led to higher conformity only when challenged by larger majorities with four confederates, but not against a single opposing confederate. This is inline with Deutsch and Gerard's explanation in relation to normative influences - i.e. in the absence of a "group" to exert normative pressures, response visibility will not show the expected effects. Furthermore, Abrams et al. [1] observed that the in-group or out-group nature of the peers - i.e. whether the peers are from the same group as the participant or not - will also mediate the effects of response visibility in physical settings. They found that when participants perceived their peers to be from the same educational background as them, public responses enhanced conformity behaviour (58\%) than private responses (33\%). Conversely, when peers were from a different educational background, conformity was lower in public responses (8\%) than in private responses $(26 \%)$. Therefore, they concluded that normative pressures resulting from public responses only emerge in relation to peers from one's own group.

Conversely, prior work have not investigated whether effects of response visibility are moderated by other conformity determinants in CMC groups. Therefore, in Chapter 7 we quantify effects of response visibility on social conformity - alongside other contextual (i.e., majority-minority group size, task objectivity, user representation, interactivity) and personal (i.e., gender, self-confidence) conformity determinants in an online group setting.

Humanness of peers: Humanness (the quality of being human or not) of peers can also determine to what extent social presence is perceived in both physical and CMC settings. Recently, several experiments have attempted to test if non-human peers such as computers, robots and virtual agents can induce conformity behaviour, similar to 
humans. For example, Brandstetter et al. [24] were amongst the first to compare how participants conform against human and robotic peers in perceptual (line judgement) and verbal tasks. The researchers observed $30 \%$ conformity against human peers in the line judgement task, whereas robots generated almost no conformity (1\%). Furthermore, while robots were seen to generate some conformity (11\%) in the verbal task this effect was not significant in comparison to what was observed in control the condition (5\% conformity). Therefore, findings of this study suggest that non-human peers may not be able to exert sufficient social pressure to trigger conformity behaviour in physical groups.

Similar findings were reported by Hertz and Wiese [77] when they replicated the line judgement task in a CMC setting, with either humans, computers or virtual agents challenging user responses. No statistically significant conformity behaviour was observed in the presence of computers or robotic peers. Midden, Ham, and Baten [120] described that the "social" nature of non-human agents is not strong enough to to generate normative influences that are essential for the line judgement task to generate conformity. Subsequently, the authors tested how increasing task difficulty in the line judgement task might impact user conformity to virtual agents and computers. They note that in high task difficulty - which appeals to informational influences - both virtual agents (21\%) and computers $(24 \%)$ generated more conformity than in the control condition (5.5\%). Hence, it was deduced that artificial majorities are capable of generating some informational conformity in CMC settings. This notion was later confirmed by Lucas et al. [108] in a survival task (rank ten pieces of art based on their importance to be saved from a hypothetical fire), where participants were seen to agree more with virtual agents who presented arguments based on facts (and hence, appealed to informational influences), than those who resorted to normative tactics to convince users to accept their ranking. Furthermore, Hertz and Wiese [78] found that non-human agents such as computers and robots can trigger conformity behaviour in analytical tasks (arithmetic sums) that benefit from informational influences, than social tasks (identifying emotion depicted on a photograph). No difference was observed for human peers in social and analytical tasks. Hence, the potential for non-human peers to create social pressure in CMC groups is apparent - especially in tasks that computers are perceived to perform better than humans.

\subsection{Personal Determinants of Social Conformity}

Despite being exposed to the same contextual factors, individuals demonstrate different rates of conformity behaviour. Crutchfield [39] attributed such individual differences in conformity behaviour to personal conformity determinants. Out of the papers analysed, $58 \%$ (64 out of 110) have investigated at least one of the personal determinants of conformity - gender $(n=38)$, age $(n=12)$, self-confidence $(n=13)$, and personality $(\mathrm{n}=28)$. The distribution of these articles across physical and CMC group settings, and the experimental paradigm used is shown in Table 2.2. In brief, 51 articles report conformity studies conducted in physical groups, 12 articles report conformity studies in CMC-based groups, and another article that investigated conformity in both physical 
and CMC settings. Furthermore, we found that these studies have used Crutchfield's experimental paradigm $(n=44)$ more often than Asch's paradigm $(n=19)$ to fabricate a group setting, with one other study using no fabrications (confederates or simulations).

\subsubsection{Gender}

"Gender" was one of the earliest personal determinants to be explored in the conformity literature [47, 91], and it is still a frequently discussed topic [139]. Interestingly, the interpretation of the term "gender" has also evolved over time in the conformity literature. More specifically, we note that the early social conformity literature (dating back to 1950's) have used the terms "gender" and "sex" interchangeably. In fact, the majority of these studies have investigated potential gender differences in users' susceptibility to social conformity influences, and explained their observations in relation to gender roles imposed by the society [39, 47, 48]. Consequently, in a vast majority of early conformity studies, "gender" is perceived as a binary variable - i.e. whether an individual identify themselves as a man or a woman. However, in more recent literature that investigate differences in susceptibility to social influences, "gender identity" is more prominently used $[101,102]$. Hence, we clarify that all experiments presented in this thesis also considered the effects of self-disclosed gender identity of participants on their susceptibility to conformity influences.

Gender is the most popularly investigated personal determinant of conformity among the papers analysed in this literature review, with $35 \%$ of the total sample $(\mathrm{n}=38)$ reporting effects of gender on conformity in both physical $(n=28)$ and CMC $(n=8)$ groups. Furthermore, two other studies have investigated gender differences in conformity across both physical and CMC settings [68,69]. We further note that 28 of these research articles used Crutchfield's paradigm, whereas another 9 studies used Asch's paradigm. In addition, another study by Wood and Karten [185] investigated effects of gender on social conformity without using confederates or simulations to create conformity pressures. More specifically, the researchers analysed differences in interaction styles of men and women who discussed a social dilemma scenario in groups of four (two men and two women) to arrive at a unanimous decision. The researchers analysed how men and women reacted to naturally occurring opposing judgements of others, to determine their conforming and non-conforming behaviour.

Initial studies that investigated effects of gender on conformity in physical groups analysed potential differences in susceptibility to conformity influences in men and women [39]. Subsequent studies investigated effects of gender on conformity in both men and women coming from different age groups [36, 47], across different experimental tasks $[3,157]$, and when challenged by both unanimous and non-unanimous majorities [4, $6,7]$ - to investigate for potential interaction effects between gender and other conformity determinants. On that note, we emphasise that these studies often used Crutchfield's paradigm to induce the perception that participants were interacting with peers from the same gender group to avoid potential confounding effects of peer gender. For instance, Allen and Crutchfield [3] and Allen and Levine [4] grouped naive participants of the same gender together and informed they will see others' responses to the experimental 
task through Crutchfield's apparatus, whereas in reality the responses shown to them via the apparatus were controlled by the experimenter.

Conversely, the studies that used Asch's paradigm have investigated whether conformity behaviour in men and women differ based on certain traits of their supposed peers. For example, Larsen et al. [99] and Fard [55] tested how experimentally induced perceptions high vs. low social status of confederates can impact conformity behaviour in men and women. Furthermore, Baron, Vandello, and Brunsman [16] tested whether the gender composition of supposed peers challenging the responses of naive participants can moderate their conformity behaviour, by exposing them to either two male confederates or one male and one female confederate. Therefore, when experimental objectives focus on traits of confederates Asch's paradigm is deemed more suitable in physical settings.

Furthermore, the effects of gender on conformity has been a recent interest in CMCbased conformity studies as well. Similar to physical groups, a majority of studies have investigated for potential gender differences in conformity by recruiting both men and women [139]. Moreover, several other studies have taken a more intensive approach by testing for effects of "partner" gender in activating gender-stereotypical perceptions of partner and self competency that can heighten conformity behaviour [101, 102]. We further note that these studies used a computer-simulated "partner" who was represented using a stereotypically gendered avatar, and hence were based on Crutchfield's paradigm.

\subsubsection{Effects of Gender on Conformity Behaviour}

Seminal conformity studies conducted in physical settings by Asch [11] and Goldberg [64] only recruited men as their experimental participants. While it is reasonable to control for gender differences in conformity in the very beginning of conformity literature, researchers later became inquisitive about how conformity would manifest in women. Crutchfield [39] conducted several iterations of his study with both young and older men and women. They observed that out of participants recruited, older women were least conforming. The author explained that older women recruited for the study came from higher socio-economic backgrounds than other participants which may have encouraged their non-conforming behaviour.

Subsequently, Larsen [98] replicated the line judgement task using both men and women as experimental participants. They note that while the overall conformity rate observed in their study (26.4\%) was much lower than what was observed in Asch's line judgement study in 1955 (36.8\%), women showed more conformity behaviour than men, with $84.6 \%$ of them conforming at least once during the experiment in comparison to $36.4 \%$ of men. Moreover, the notion that women conform more than men in physical groups has also been tested across different types of experimental tasks. For instance, Endler, Minden, and North [52] reported higher conformity in women than men in visual perceptual and informational tasks. Similar observations were reported by Santee and Maslach [148] where women were seen to conform more often than men to a simulated majority's responses in a social dilemma task. Moreover, Costanzo and Shaw [36] showed that these gender differences in conformity also persist across different age groups by reproducing the line judgement task in participants aged 7-21 years. The authors reported that women showed higher conformity (between 31\%-38\%) than men (between 28\%-31\%) 
- regardless of their age. Furthermore, in contrast to the above studies that analysed effects of gender on conformity in unanimous majorities, Allen and Levine [6] analysed how men and women would respond to conformity influences coming from non-unanimous majorities. The authors compared conformity behaviour in men and women as their responses to a series of visual perceptual, informational and opinion-based tasks were challenged by either a unanimous majority or a non-unanimous majority (one dissenter other than the naive participant). They observed that while previously seen gender differences in conformity manifest with unanimous majorities - with women conforming more often than men - these differences were absent when participants were challenged by non-unanimous majorities.

Consequently, as gender effects on conformity became more apparent researchers looked into possible explanations for this phenomenon. For instance, Endler, Minden, and North [52] explained the observed gender differences in conformity as a result of different cultural expectations that guide social behaviour in men and women as previously described by Krech, Crutchfield, and Ballachey [93]. More specifically, they describe that masculine gender roles encourage independence, whereas feminine gender roles encourage group dependence and cooperation with others, hence leading to lower and higher conformity respectively. This argument was empirically supported by Eagly, Wood, and Fishbaugh [48] in a later study, where authors observed that women conformed more than men only when their responses were under surveillance by other group members. Conversely, men were seen to conform less under surveillance than in the absence of surveillance. The authors explained that both men and women adjusted their conforming behaviour to suit the gender roles imposed on them when placed under surveillance, but deviated from gender-specific behaviour in the absence of surveillance.

Moreover, several studies have analysed gender differences in conformity behaviour in relation to perceived self/peer competency. For example, a study by Wood and Karten [185] required men and women to rate self and peer competency after completing a discussion task in a mixed gender group (two men and two women). The authors used no simulations or confederates in this study and instead allowed conformity pressures to manifest naturally by asking all group members to agree on a single decision for a social dilemma scenario. Their findings indicated that women were more likely than men to adjust their opinions to agree with others in the group. The authors further emphasised that the self and peer ratings of participants showed that men were perceived to be more competent than women by themselves and other group members - which may have led to gender differences in conformity behaviour. Wood and Karten repeated the experiment, where they experimentally induced perceived competency of self in relation to peers by informing participants of their intellectual aptitude in comparison to other group members before the discussion task. In this setup, only perceived self competency had an impact on conformity such that those who perceived themselves to be less competent than the group conformed more in comparison to those who perceived themselves to be more competent than the group. More specifically, when competency of group members was apparent, previously seen gender effects on conformity diminished. Conversely, studies by Allen and Crutchfield [3] and Endler et al. [53] have shown that while being informed that the "group" is more competent than themselves can heighten conformity in both men and women, the increase in conformity is often higher for women. 
Furthermore, other studies report that perceived social status of self and peers in a group can also moderate effects of gender on social conformity. This notion was investigated by Fard [55] where half of the experimental participants (both men and women) were led to believe they were superior to their group members who were introduced as unemployed individuals and salesmen, whereas the other half of participants perceived themselves to be inferior to their peers whom they presumed to be university lecturers and doctoral students. The authors highlighted that women conformed more than men only when they presumed their peers to be superior than them. Conversely, women and men showed no significant differences in conformity when confronted by peers whom they perceived to be inferior to them. Alternatively, Larsen et al. [99] present slightly different findings with regard to social status and gender effects on conformity. They note that when confronted by "high status" peers, men conformed more than women as a result of their focus on goal-achievement. Conversely, in the absence of experimentally induced peer superiority (i.e. participants perceived themselves to be similar to other group members), women conformed more than men as a result of their focus on maintaining interpersonal relations.

We further note some effort in physical conformity studies to investigate effects of confederate gender on conformity behaviour of naive participants - without experimentally inducing high vs. low perceptions of their competency and social status. For example, Barron [17] exposed participants to either two male confederates or one male and one female confederate to see how the gender composition of the confederates impact user conformity behaviour in a memory judgement task. However, the authors reported no statistically significant differences in user conformity behaviour against the two confederate gender compositions tested. We did not come across other studies that have investigated effects of confederates' gender group composition on user conformity in physical settings.

The first study to investigate gender differences in conformity behaviour in CMC settings was report by Guadagno and Cialdini [68]. More specifically, the authors compared how receptive men and women are to their correspondents through faceto-face and email. Participants were required to engage in a discussion with a same gender confederate on a predetermined topic of societal interest. The naive participant acted as the interviewer who asked predetermined questions from the confederate who provided answers based on a script. This approach allowed the researchers to measure conformity in terms of attitude change in the naive participant after the discussion, while controlling for effects from peer argument strength. The authors emphasised that women were more receptive to their opposing partners in face-to-face interactions than in email-based interactions. Alternatively, men showed no differences in receptiveness to their peers over the two communication mediums. The authors rationalised these gender differences in conformity across face-to-face and CMC-based communication mediums in terms of gender roles imposed by the society (as previously described by Endler, Minden, and North [52] and Eagly, Wood, and Fishbaugh [48]). In other words, authors describe that women who often focus on relationship formation and cooperation, align their attitudes with those of their opposing partner more often in face-to-face interactions where relationship goals are more salient and attainable. Alternatively, men whose social roles focus on independence and agency are not affected by differences in social 
constraints between the two communication mediums. A subsequent study by Guadagno and Cialdini [69] further corroborated these observations and highlighted that observed gender differences in conformity in face-to-face and CMC settings are not a result of disparities in computer literacy between men and women.

Subsequent CMC-based conformity studies attempted to replicate gender effects previously observed in physical groups - across a variety of experimental tasks. For example, Zhu, Huberman, and Luon [188] investigated potential gender differences in how men and women conform to peer choices in an online preference-based task. Similarly, Rosander and Eriksson [139] analysed potential gender differences in conformity behaviour as participants completed an online MCQ quiz that contained informational and logical questions. However, in contrast to observations in physical groups, these studies did not observe significant gender differences in conformity. Furthermore, effects of gender on conformity have also been investigated with relation to task difficulty in CMC groups by Rosander and Eriksson [139]. They highlighted that conformity in both men and women heightened equally as a result of increasing task difficulty in informational and logical questions - indicating no significant gender differences in conformity.

Nevertheless, these findings are insufficient to provide conclusive evidence of gender differences in social conformity behaviour in CMC settings. Hence, in the studies presented in Chapters 4, 5, 6, 7 and 8 we account for user gender as a determinant of conformity in the presence of other contextual (i.e., majority-minority group size, task objectivity, social presence) and personal (i.e., age, self-confidence, personality) determinants of conformity to further investigate this matter.

Furthermore, a significant proportion of gender-based CMC conformity studies have investigated the impact of gender-stereotypical perceptions of self and peer competency on online conformity behaviour. For instance, Lee [101] tested how men and women responded to incorrect judgements of a "partner" that was represented using a stereotypically masculine or feminine representation. The experiment required the participants to complete a MCQ quiz containing both stereotypically masculine and feminine tasks (questions based on sports vs. fashion), where they indicated their initial and final answers to each question both before and after seeing partner's responses. The authors emphasise that men and women were less inclined to conform to their partner in stereotypically masculine (sports) and feminine (fashion) questions respectively - that they perceived to be well-known to their own gender group. In addition, the authors observed that men were more receptive to conformity influences in stereotypically feminine questions when challenged by a partner represented using a stereotypically feminine user representation. Similarly, women were seen to conform more in stereotypically masculine questions when the opposing partner was represented using a stereotypically masculine user representation. Therefore, the authors concluded that in the presence of genderstereotypical tasks, both men and women perceived competency of themselves and their peers based on available gender cues - which also determined whether an individual would conform to their partner or not in CMC groups. Furthermore, they rationalised these findings in relation to participants' susceptibility to informational influences. More specifically, they explained that when individuals stereotypically perceive a task to favour one gender over the other, they are more inclined to accept information coming from the 
favoured gender group because they assume such gender-stereotypical conformity will improve their chances of being "correct".

However, prior work investigated effects of gender and related stereotypes in the presence of a single opposing "partner". Hence, in Chapter 6 we present a study that analyse effects of peer gender in larger groups with more than one peer. More specifically, we expose participants to different majority and minority gender group compositions, as they complete gender stereotypical tasks. Additionally, we compare how two different stereotypical gender cues (i.e. gendered avatars and names) differ in how strongly they trigger gender-stereotypical perceptions in CMC groups. Furthermore, we also investigate whether observed gender stereotypical perceptions of peer competency are reliable and can lead to correct answers as participants expect, to empirically determine outcomes of gender stereotypical conformity in CMC settings.

\subsubsection{Age}

Effects of age on conformity behaviour is one of the less frequently investigated personal determinants in the existing literature. We found only 12 papers that tested for effects of age in the sample considered for this literature review (i.e. $11 \%$ of the total sample) - with 11 of them reporting findings in relation to physical conformity studies. Only one study by Zhu, Huberman, and Luon [188] have accounted for effects of age on conformity in online groups. We emphasise that only studies that have analysed effects of age within the same experiment (by recruiting participants from different age groups) were used for this analysis.

Moreover, we note that the majority of the studies that tested for effects of age on conformity have used Crutchfield's paradigm $(n=9)$. Further analysis showed that these studies primarily tested for effects of user age on conformity by recruiting participants from different age groups (e.g., Pasupathi [132] compared conformity behaviour between younger and older adults, aged 18-35 years and 63-86 years respectively). Therefore, using a Crutchfield's apparatus to simulate group responses was deemed suitable to avoid confounding effects that can be introduced by having live confederates (e.g., gender/age of confederates). Alternatively, studies that used Asch's paradigm have investigated effects of confederates' age on user conformity [94], compared conformity behaviour in different age groups against human vs. robotic peers [172], or recruited young school children (about 3 years old) who might have difficulty interacting with simulated majorities [175] - which required them to use live confederates to generate conformity pressures.

\subsubsection{Effects of Age on Conformity Behaviour}

Initial studies that investigated effects of age on conformity behaviour were primarily focused on children, adolescents and young adults. For instance, Costanzo and Shaw [36] who was one of the first to empirically investigate age differences in conformity - recruited participants aged 7-21 years to analyse age differences in conformity across four age groups - 7-9, 11-13, 15-17 and 19-21 years - using the classic line judgement task. The authors observed a non-linear relationship between user age and conformity such that conformity behaviour increased till preadolescence, after which it was seen to decrease 
through adolescence and early adulthood. These observations were also confirmed by Costanzo, Reitan, and Shaw [35] where conformity rates of 33\%, 50\%, 45\% and 35\% were observed across the above four age groups respectively. Furthermore, these studies explained the age differences in conformity behaviour as a result of the socialisation processes that we face from early childhood till young adulthood. They described that children in early development stages (7-9 years) are not fully aware of the social pressure to conform, until the onset of preadolescence. During preadolescence (9-13 years) they become aware of their social peers and heavily rely on them to determine own behaviour - which significantly enhances their receptiveness to conformity influences. Subsequently, by post-adolescence and early adulthood years (13-21 years) they become more confident about their own judgements which results in a decline in conformity behaviour. However, as a result of their knowledge on undesirable outcomes of nonconformity, conformity is still higher than what was observed in early childhood years.

Interestingly, subsequent studies showed slightly different observations with regard to age and conformity in children and adolescents. For instance, Allen and Newtson [8] note that conformity behaviour gradually reduced in children from grades 1,4 , and 7 , after which it increased in students from grade 10. They further note that this trend persist in both visual perceptual and opinion-based experimental tasks. Moreover, another study by Walker and Andrade [175] that investigated effects of user age on conformity across school children and adolescents across 3-5, 6-8, 9-11, 12-14 and 15-17 years reported diminishing conformity rates - 85\%, 42\% 38\%, 9\% and $0 \%$ - also indicating declining susceptibility to conformity influences as children age. Therefore, how susceptibility to conformity pressures change in young people is still unclear in physical settings. Furthermore, none of the studies analysed in this literature review investigated effects of age on conformity with regard to children in CMC settings.

Moreover, several other studies have also tested for effects of age in young and older adults. For example, Klein [91] found that older participants (60-86 years) conformed significantly more often than younger participants (16-21 years) in a visual perceptual task. They further note that while increasing task difficulty heightened conformity behaviour in both age groups, the increase in conformity was much higher in older participants than in their younger counterpart. Conversely, Pasupathi [132] found that young adults (18-35 years) conformed more often than older adults (63-86 years) especially in emotional tasks (e.g. labelling human emotions) than in non-emotional tasks (e.g. labelling geometric shapes). The authors compared their results against Klein's findings to emphasise that user age can interact with other determinants such as task type and task difficulty to show different effects on conformity behaviour.

On a different note, Kumar [94] investigated how age of the confederates (9-10 years vs. 14-15 years) that challenge user responses impact conformity behaviour in preadolescent (9-10 years) and adolescent (14-15 years) users in a visual perceptual task. Furthermore, the authors used the Asch's paradigm to manipulate the size of the opposing majority such that naive users were challenged by either five or ten confederates (small vs. large majority group size). Their findings indicated that age of the confederates moderated effects of both majority group size and user age. More specifically, the authors note that larger majorities with younger confederates (9-10 years) heightened user conformity, an increase in majority size did not produce more conformity with older 
confederates (14-15 years). Moreover, it was also noted that users were more likely to conform when opposing confederates were older or equal in age to them than when the confederates were younger. Based on these findings, Kumar [94] emphasised that the impact of age on conformity should be analysed accounting for potential interaction effects with other contextual and personal determinants.

However, despite the reported effects of age on conformity in physical groups, researchers are yet to thoroughly investigate whether and how age moderates conformity behaviour in CMC groups. Out of the papers analysed in this survey, only one study by Zhu, Huberman, and Luon [188] accounted for age differences in conformity in a CMC environment. They deployed a large scale online survey where 433 participants - aged 18-82 years ( $M=27$ years) - were asked to indicate their personal preferences between pairs of photographs, both with and without knowledge of others' preferences. However, while the results of this study indicated conformity rates between $14.1 \%-32.5 \%$, the authors reported no statistically significant age differences in conformity behaviour.

Nevertheless, findings of prior work with regard to effects of age on conformity in physical groups encourage further investigation into potential effects of age on conformity in CMC groups across different experimental tasks and group compositions. Hence, in Chapter 5 we investigate effects of age on user conformity behaviour in an Instant Messaging platform, across three aspects - user age, age group composition of the "peers" challenging user judgements and stereotypically age-biased tasks.

\subsubsection{Self-confidence}

As per Deutsch and Gerard [42], people often conform to the majority when they are unsure of the "correct" response to an ambiguous situation (informational conformity). In other words, the level of self-confidence an individual has on their personal judgement has a significant impact on their decision to conform (or not) to the majority, forsaking their own judgement. Thus, in conformity literature, self-confidence has been tested from two perspectives - self-confidence of the naive participant and self-confidence of opposing peers. On one hand, self-confidence of participants can determine how susceptible they are to conformity pressures. On the other hand, self-confidence of peers - if this information is available - can be an indicator of the accuracy of their judgements, which in turn can determine the perceived strength of social influence they pose on a subject. Moreover, in the research articles analysed we observe a total of 13 studies (12\% of the total sample) that report effects of self-confidence on conformity in physical ( $\mathrm{n}=$ 11) and CMC $(n=3)$ group settings, using both Crutchfield's paradigm $(n=7)$ and Asch's paradigm $(\mathrm{n}=6)$.

A majority of conformity studies that tested effects of self-confidence in physical groups did so by experimentally manipulating how participants perceived their competency within the group. For example, Smith [160] manipulated perceived competency of both the naive participant and two confederates in a visual perception task, by reporting the accuracy of their individual performances in pre-test trials. To induce higher confidence, half of the participants were told that they scored 95, when the confederates supposedly scored 73 and 54 . Alternatively, to induce lower self-confidence participants were told they scored 54 when their two peers scored 73 and 95 . A similar approach was 
used by Rosenberg [142] in a line estimation task, to manipulate self-confidence of naive participants. They asked all group members to complete ten pre-test line judgement trials after which the naive participant was informed that they either obtained more correct answers than their peers, or not. Furthermore, the presence of non-verbal cues in face-to-face interactions have been used to trigger perceptions of peer confidence in conformity studies. For instance, in a study by Baron, Vandello, and Brunsman [16] confederates responded to a memory judgement task with no delay, in a confident tone of voice, with no pauses or filler words in their responses to insinuate high confidence. Conversely, they acted hesitant, expressed uncertainty and used verbal qualifiers (e.g. somewhat) in their responses to show low confidence. By manipulating the perceived competency of self and/or peers, these studies were able to investigate how confidence in one's own ability compares with perceived confidence of others to determine conformity behaviour.

In CMC settings, the effect of self-confidence on conformity has been tested differently. A majority of studies required participants to self-report their confidence in initial personal judgement on a scale. For example, in a study by Lee [102] participants indicated their initial answer and confidence in the chosen answer (or initial confidence) on a 10-point scale - for each question in an online quiz - after which they were exposed to the answer of their partner. We further note that participants of this study were not exposed to the confidence ratings of their group members to avoid confounding effects.

\subsubsection{Effects of Confidence on Conformity Behaviour}

In physical studies that manipulated self-confidence of participants by experimentally inducing high or low perceptions of self and peer competency, we found a consistent relationship between conformity and confidence. Smith [160] showed that in a simple visual perceptual task, participants who perceived themselves to be more competent than their peers, denoted more confidence in their answers and were less likely to conform to peer judgements. Conversely, those who were led to believe that their peers are more competent than them were seen to conform more often. These findings were later confirmed by Rosenberg [142], who described conformity as a function of self and peer confidence. They explain that a negative difference between confidence in own answer and confidence in peer answers lead to conforming behaviour, where as a positive difference between the two factors would discourage conforming behaviour. Furthermore, Baron, Vandello, and Brunsman [16] observed that participants are more likely to conform to peers who project high levels of confidence through verbal and non-verbal cues, than those who do not in a memory judgement task.

Furthermore, as seen in the studies above, experimentally inducing perceived competency by presenting participants with fabricated performance results on a series of pre-test visual perceptual tasks (e.g., [119, 142, 160]) can effectively influence how confident participants are of their own responses and of their peers' in a subsequent visual perceptual experimental task. In addition, Endler et al. [53] showed that these experimentally induced perceptions of competency can also be transferred from one task type to another. In this study, competencies of the naive participant and the group were reported based on their supposed performance in an informational task (completing 
the Canadian Knowledge Inventory [54]). Subsequently, the same group completed a different informational task (MCQ quiz on general knowledge) and a visual perceptual task where their conformity behaviour was analysed. The researchers that experimentally induced perceptions of self and peer competency generalised from an informational task (i.e. the Canadian Knowledge Inventory) to another informational task as well as a visual perceptual task. In other words, regardless of the nature of the task where their performance was evaluated and reported, participants who were led to believe that they are more competent than the group, conformed less in the subsequent informational and visual perceptual tasks, and vice versa.

On a different note, we highlight that none of the studies that investigated effects of confidence on conformity in physical groups used experimental tasks that are subjective in nature. Therefore, current findings are insufficient to determine how confidence can mediate conformity effects in subjective content.

In CMC-based conformity studies there has been some effort to investigate effects of self-confidence in visual perceptual tasks. For instance, Kyrlitsias and Michael Grigoriou [96] analysed how self-reported confidence levels of participants determine their conformity behaviour in a replication of Asch's line judgement task in a classroom created using Virtual Reality (VR). As self/peer competency was not manipulated experimentally (as in studies described before), participants were not exposed to confidence ratings of their peers. Their findings indicate that participants who reported lower confidence on their judgements conformed more to an incorrect majority - as previously seen in physical groups $[119,142,160]$.

Furthermore, the effects of self-confidence on conformity can sometimes be moderated by other personal determinants, such as user gender in CMC groups. For example, Lee [102] found that self-reported confidence on personal answer showed the expected relationship with conformity behaviour in women, but not in men. They note that men reported higher confidence on their initial judgements regardless of their subsequent conforming or non-conforming behaviour. Thus, they argue self-confidence is not a reliable measurement of conforming behaviour in men, and that more work is needed to investigate what other personal and contextual factors moderate effects of confidence on conformity in CMC groups.

Therefore, in Chapters 4, 5, 6, 7 and 8 we investigate effects of self-confidence on user conformity across different objective and subjective experimental tasks (i.e., factual, logical and opinion-based), and alongside other contextual and personal determinants of conformity to further understand where self-confidence can reliably determine user conformity in CMC groups.

\subsubsection{Personality}

Conformity studies have attempted to capture the effect of an individual's personality on their susceptibility to conformity pressure in physical groups since the early 1950s [39, $50,114]$. Crutchfield [39] was one of the first researchers to investigate if personality differences among participants make them more or less susceptible to conformity influences. They were motivated to do so after observing significant individual differences in conformity behaviour among their participants who were recruited from a fairly 
homogeneous sample (all men with similar professional backgrounds). They observed that at the lower end several participants conformed only once or twice, whereas at the upper end some others conformed in 17 out of 21 critical trials. Crutchfield argued that these variations in conformity are a result of differences in personal susceptibility to conformity influences and hence identifying what personality traits separate high conformers from low conformers is vital to better understand conformity behaviour. Consequently, we observe 28 studies ( $25 \%$ of the total sample) that have investigated effects of different personality traits on social conformity behaviour. A majority of these studies are based on physical groups $(\mathrm{n}=23)$, whereas only 5 studies investigate personality as a conformity determinant in CMC groups. We further note that Crutchfield's paradigm has been used more often $(n=21)$ than Asch's paradigm $(n=7)$ for this purpose.

To provide a meaningful overview of the literature analysed, we present findings across seven dimensions which includes six prominently researched personality traits (i.e., ascendancy vs. submission, need for affiliation vs. achievement, (social) anxiety, self-esteem, ego, need for public individuation) and a personality taxonomy (i.e. John and Srivastava's Big-five Traits [84]). Furthermore, we note that the six personality traits mentioned above have been captured using different personality inventories in the analysed literature. Hence, we will next describe what each personality trait attempts to quantify, along with the instruments that have been used.

Ascendancy describes an individual's desire to be prominent in group situations, to assert oneself and to show authority over others. Conversely, those with low need for ascendancy are described to as "submissive" [123]. We further note that ascendancy and submissive personality traits have only been investigated with regard to conformity in physical groups $[18,123]$. Moreover, all these studies have used Allport's AS Reaction Study [9] to capture ascendancy-submissive nature of their participants.

Furthermore, need for affiliation (also referred to as affiliation motivation) describes the human desire to be accepted and well-thought of in groups, and their tendency to believe that groups reject those who deviate from group norms [72]. Alternatively, need for achievement (or achievement motivation) encourages people to perform well and be successful in group settings. In other words, affiliation and achievement motivations respectively appeal to normative and informational influences that trigger conformity behaviour [157]. Being two of the more frequently investigated personality traits in physical conformity studies, affiliation and achievement motivations of individuals have been quantified using three distinct personality inventories namely - the MarloweCrowne Social Desirability scale (M-C SD) [38], the Edwards Personal Preference Schedule (EPPS) [49], and the Thematic Apperception Test (TAT) [13].

Moreover, social anxiety has been analysed in relation to conformity behaviour in both physical $[112,119,148]$ and CMC-based [134] conformity studies. In simple terms, socially anxious individuals feel nervous and uneasy in social pressure situations especially when the appropriate response is unclear. Furthermore, unhealthy levels of public self-consciousness (heightened sense of awareness of one's social identity and other people's reaction to it) can also lead to social anxiety. The effects of social anxiety on social conformity have been investigated using the Test Anxiety Scale (TAS) [109], the Public and Private Self-consciousness and Social Anxiety Scale [57], and the State-Trait Anxiety Inventory for Adults scale (STAI-AD) [163]. 
Self-esteem encompasses how individuals evaluate their self-worth. Individuals who display high self-esteem are often confident of their own abilities. Furthermore, Costanzo [34] described that tendency to "self-blame" - or the attribution of blame to self - is a logical consequence of low self-esteem. They explained that individuals who tend to think poorly of themselves (low self-esteem) would evaluate their actions harshly particularly if these actions deviate from the standards of the group. Self-esteem has been analysed using the Texas Social Behavior Inventory (TSBI) [76] and the Rosenberg's Self-Esteem Scale (RSE) [143] in both physical [112, 148] and CMC groups [134], while the effects of self-blame has been evaluated using the Costanzo's Self Blame Test (SB) [34] in physical conformity studies.

Furthermore, how an individual perceive their self-importance in comparison to others is described as their ego strength. Those who demonstrate high ego strength perceive themselves to be superior than others and hence can absorb contradictory informational input coming from peers without being threatened [160]. In the studies analysed, ego strength or ego was one of the first personality traits to be analysed in physical groups $[39,160]$. These studies used two distinct personality inventories to quantify an individual's ego strength - the Minnesota Multiphasic Personality Inventory (MMPI) [75] and the Barron's Ego Strength Scale [17].

In 1985, Maslach, Stapp, and Santee [113] described public individuation as an individual's "willingness to engage in behaviours that would publicly differentiate themselves from others". In other words, those with a high need for public individuation try to act conspicuously to make themselves stand out in group settings. Therefore, such individuals can be less responsive to normative conformity pressures [161]. The effects of disposition for public individuation on conformity have been analysed in both physical [112, 148] and CMC [104] groups using the Individuation Scale [113].

More recent conformity studies investigating effects of personality in CMC groups have used John and Srivastava's Big-five Traits [84] to capture personality of their participants $[130,134]$. This taxonomy aims to quantify personality across five traits namely - Openness, Conscientiousness, Extraversion, Agreeableness and Neuroticism (OCEAN). In simple terms, those who show high openness are receptive to new ideas and often seek out new experiences, whereas those who prefer consistency and caution tend to rate lower in this trait. Alternatively, conscientiousness measures our desire to perform well in a task. Therefore, individuals who display high conscientiousness tend to be goal-oriented, organised and diligent in their work, whereas those who display low conscientiousness are often described as laid-back and relaxed. Furthermore, our tendency to be outgoing, energetic and talkative is measured by the extraversion trait. Those who rate higher in the extraversion scale prefer social interactions over being solitary, and are eager to offer their opinions and suggestions. Moreover, agreeableness measures our tendency to be kind, cooperative, and considerate of others. At the upper end of the scale are those who demonstrate high levels of empathy and altruism, and prioritise social harmony and cooperation. Those who lack empathy and concern for social harmony tend to score low agreeableness scores. Lastly, neuroticism is described as the "disposition to experience negative affects, including anger, anxiety, self-consciousness, irritability, emotional instability, and depression" in Psychiatry [177]. In other words, those who rate low in neuroticism tend to be self-confident and resilient against social pressures. 


\subsubsection{Effects of Personality on Conformity Behaviour}

We now report findings of our analysis on effects of each of the aforementioned personality traits on social conformity behaviour. We emphasise that even when a personality trait has been measured using the same personality inventory across different studies, their findings can differ and should therefore be interpreted accounting for other contextual (e.g. experimental task) and personal (e.g. gender) variables.

Ascendancy vs. submissiveness: The first observation with regard to ascendancy and conformity was reported by Crutchfield [39]. Their findings indicate a negative correlation between the "leadership ability" of men and their susceptibility to conformity influences in both objective and subjective experimental tasks. It was also observed that individuals who rated themselves as "effective leaders" who often "take an ascendant role in relations with others" were seen to be more independent in their judgements. This notion was further investigated by Beloff [18] in a opinion-based experimental task where both men and women were categorised as either ascendant or submissive - based on their score in the AS Reaction survey [9]. The authors note that while high disposition to ascendancy reduced conformity in men, these effects did not replicate in women. They concluded that effects of ascendancy on conformity are therefore complicated by gender. Furthermore, another study by Mouton, Blake, and Olmstead [123] showed that effects of disclosing personal identity on conformity behaviour in physical groups are moderated by the ascendant or submissive nature of participants. Disclosing personal identity heightened conformity tendencies only in submissive participants (in comparison to when they were anonymous), whereas ascendants showed no differences in conformity regardless of whether their identity was disclosed to the group or not. The authors concluded that submissive individuals are more susceptible to conformity influences than ascendants - especially when they can be individually criticised for their personal opinions. Thus, the cumulative findings of prior work indicate an inverse relationship between ascendancy of an individual and their conformity behaviour - effects of which can be moderated by other contextual and personal conformity determinants, such as social presence and gender. However, the existing literature is insufficient to determine if these findings would replicate in CMC-based groups.

Affiliation vs. achievement motivation: Effects of social approval or affiliation motivation of individuals on their conformity behaviour has been tested across a variety of experimental tasks. For example, Strickland and Crowne [167] investigated effects of affiliation motivation on social conformity in a auditory perceptual task, deployed using Crutchfield's paradigm. They used the Marlowe-Crowne Social Desirability (M-C SD) scale [38] to categorise their participants in to two groups - low vs. high need for affiliation. The authors note that participants with high need for affiliation were significantly more likely to conform to majority's responses (56\% average conformity), than those who demonstrated low affiliation needs (34\% average conformity). Strickland and Crowne [167] explained that participants who showed high affiliation needs were motivated to "go along with others" in cases of conflict, to satisfy their desire for social approval. These findings were confirmed by McGhee and Teevan [115] in a visual perceptual task which also employed Crutchfield's paradigm. They used the Thematic Apperception Test (TAT) [13] to measure affiliation motivation of their participants - in contrast to the $M-C$ 
DS scale used by Strickland and Crowne [167]. Despite the differences in the instruments used, the authors observed the inverse relationship between need for affiliation and conformity behaviour - which was previously observed by Strickland and Crowne [167].

Moreover, Hardy [72] investigated effects of affiliation motivation on conformity when challenged by both unanimous and non-unanimous majorities in physical groups. They used the Thematic Apperception Test (TAT) [13] to measure and categorise their participants' affiliation motives as low, medium or high. Participants were required to indicate their opinions on divorce, which were then challenged by a unanimous or a non-unanimous majority of confederates. In non-unanimous cases, naive participant's opinion was supported by another confederate (social support condition). The authors observed that when challenged by a unanimous majority, participants who showed low affiliation motivation conformed significantly less than those with medium or high affiliation motivation - which resonates with prior observations in this regard [115, 167]. However, in the presence of a partner who supported their opinion those with low affiliation needs were seen to conform more than when opposed by a unanimous majority. In other words, being exposed to both sides of an argument was seen to trigger more conformity in those with low affiliation needs. Conversely, the presence of a partner either diminished or had no effects on conformity behaviour of participants with high/medium affiliation needs. Hardy [72] rationalised that to those who conform to gain social approval, conforming to a non-unanimous majority is less appealing.

Furthermore, a study by Sistrunk and McDavid [157] showed how effects of affiliation and achievement motivations on conformity are moderated by task difficulty in perceptual experimental tasks. They used the Edwards Personal Preference Schedule (EPPS) [49] to measure both high/low affiliation and achievement motivations of individuals. Task difficulty was categorised as "easy" or "difficult" based on how their control group completed the experimental task set. The authors observed an interaction effect between achievement motivation and task difficulty, in addition to an inverse relationship between affiliation motivation and conformity which has been previously seen by Strickland and Crowne [167] and McGhee and Teevan [115]. More specifically, in "easy" tasks those with high achievement needs were less prone to conformity behaviour than those with low achievement needs, whereas in "difficult" experimental tasks high and low achievers showed no significant differences in conformity behaviour. They further note that those with high affiliation needs conformed more frequently than those with low affiliation needs regardless of task difficulty. Therefore, observations from Hardy [72] and Sistrunk and McDavid [157] confirm that effects of affiliation and achievement motivation on conformity can be moderated by other contextual determinants.

Social anxiety: In 1967, Meunier and Rule [119] investigated the effects of anxiety on conformity behaviour using Asch's line judgement task. All participants completed the Test Anxiety Scale (TAS) [109] - which scores were then used to categorise participants into two groups based on their low vs. high susceptibility to anxiety. The authors then compared conformity behaviour between the two groups to highlight that those who indicated high anxiety levels showed more conformity behaviour ( $26 \%$ conformity) than those who indicated low anxiety levels (11\% conformity).

Subsequently, Santee and Maslach [148] investigated effects of social anxiety and selfconsciousness using Fenigstein, Scheier, and Buss's Public and Private Self-consciousness 
and Social Anxiety Scale [57]. Participants were first administered the personality test, after which they were asked to indicate their responses to a series of social dilemma tasks. They could then either choose one of three potential responses provided by the experimenters or provide an entirely new response (referred to as "creative dissent"), after being exposed to either unanimously opposing or split majorities. The researchers found that social anxiety and public self-consciousness showed significant effects on conformity and dissenting behaviour respectively - but only when challenged by unanimous majorities. More specifically, they found that participants who showed high social anxiety displayed more conformity behaviour, whereas those who demonstrated low social anxiety and low public self-consciousness were more likely to engage in creative dissent (present a new opinion). Santee and Maslach [148] explained that socially anxious individuals often feel uncomfortable in social pressure scenarios (as the one induced in this experiment) which can heighten their susceptibility to normative conformity. Conversely, those who are less conscious of their social identity are more likely to be comfortable with presenting a new opinion. They further note that split majorities did not generate sufficient influence for similar correlations to appear between personality traits and conformity. These findings were later confirmed in a similar experiment by Maslach, Santee, and Wade [112].

Moreover, effects of anxiety on conformity have also been investigated in CMC settings. For instance, Perfumi et al. [134] used the State-Trait Anxiety Inventory for Adults scale (STAI-AD) [163] to test effects of anxiety on conformity behaviour in a $\mathrm{CMC}$-based replication of the line judgement task and a series of vocabulary tests. The authors observed a positive correlation between conformity and anxiety such that those who rated higher scores in the STAI-AD scale were reported to be more inclined towards conformity behaviour than others. Thus, the effects of anxiety on conformity behaviour has been found to be consistent across both physical and CMC groups.

Self-esteem: Janis and Field [83] indicated that individuals with low self-esteem are more persuadable than those with higher self-regard, using the Persuasibility Test (a paper based survey). This was later confirmed by Santee and Maslach [148] in a social dilemma task, where self-esteem was captured using the Texas Social Behavior Inventory (TSBI) [76]. They showed that individuals with lower self-esteem tend to perceive themselves with low regard in comparison to their peers and hence are more vulnerable to social conformity influences. Furthermore, Costanzo [34] analysed how the amount of blame individuals placed on themselves (self-blame) and their peers (otherblame) impact their conformity behaviour using a Self Blame (SB) test. The authors observed a positive correlation of 0.54 between the self-blame and conformity - which indicated that those who thought poorly of themselves were more prone to conformity than others. Furthermore, a positive correlation of 0.63 was observed between conformity and the difference between self-blame and other-blame - which consequently led the authors to describe conformity behaviour as a function of how much blame people place on themselves and others.

More recently, Perfumi et al. [134] attempted to quantify effects of self-esteem on conformity in CMC-groups, using Rosenberg's Self-Esteem Scale (RSE) [143]. However, the authors did not observe any significant relationship between participants' self-esteem and their conformity behaviour. Therefore, the relationship between self-esteem and 
conformity observed in physical groups is yet to be replicated in CMC-based groups.

Ego: Effects of an individual's ego on their conformity behaviour was one of the personality traits that Crutchfield investigated in their seminal study. The authors used Barron's Ego Strength scale (also known as E scale) [17] to measure ego strength of their participants. They reported an inverse correlation (-0.33) between the two factors as participants who showed high ego strength were seen less likely to conform to seemingly incorrect majorities. Furthermore, Smith [160] investigated effects of ego strength on conformity behaviour from two perspectives. On one hand, they attempted to replicate Crutchfield's findings by recruiting individuals who rate low and high ego strength on the Minnesota Multiphasic Personality Inventory (MMPI) [75]. On the other hand, they also investigated how experimentally inducing low and high perceptions of self-valuation in participants - by informing that their peers are more accurate than them or vice versa - can impact their conformity behaviour. The authors reported significant effects from the experimentally induced self-valuation, whereas no effects were observed from ego strength captured through the personality inventory. Participants who perceived themselves to be more competent in the task than their peers were less likely to be receptive to peer judgements than those who perceived their peers to be more competent than them - regardless of their ego strength. Therefore, the authors concluded that effects of experimentally induced self and peer competency may have surpassed potential effects of ego strength on conformity behaviour. We did not find any study that investigated the effects of ego strength on conformity in CMC groups.

Public individuation: The need for individuation - i.e. to differentiate oneself from others in group settings - has been investigated with regard to conformity in both physical and CMC settings. For example, Santee and Maslach [148] tested effects of individuation on conformity in a social dilemma task using Maslach, Stapp, and Santee's Individuation Scale [113] to measure the need for public individuation in experimental participants. Their findings indicate an inverse relationship between the need for individuation and conformity behaviour. The authors explained that non-conforming behaviour in an opportunity to publicly differentiate oneself from a unanimous group majority and therefore is appealing to those who display high need for individuation. Conversely, those who show low individuation tendencies are more concerned about fitting in with others, which can encourage more conformity behaviour. Furthermore, Maslach, Santee, and Wade [112] reported that an individual's need for individuation has positive correlation with their propensity to creatively dissent from the majority's response in a similar social dilemma task. Therefore, in physical groups individuation is consistently seen to reduce inclination to conform.

In a more recent study, Lee [104] investigated how need for public individuation (measured using Maslach, Stapp, and Santee's Individuation Scale [113]) moderate the impact of individuating information on conformity in CMC settings. Participants in individuated conditions were requested to introduce themselves to their peers by mentioning their age, hobby, favourite colour and TV show before progressing to the experimental task. The authors note that introductions were a way of insinuating a sense of personalisation among the participants. Conversely, participants who were assigned to non-individuated conditions remained anonymous throughout the experiment. The findings from this study indicate that participants who showed high need for individuation 
were less likely to conform in the individuated condition - where their identities were personalised through introductions - in comparison to the anonymous condition with no personalisation. However, the presence or the absence of individuating information had no effect on the conformity behaviour of participants who showed low need for individuation. Therefore, authors concluded that the impact of an individual's need for individuation on their conformity behaviour can be enhanced in CMC settings by allowing for personalisation.

Big-five traits: John and Srivastava's Big-five personality inventory is increasingly been used to measure personality of individuals in Social Psychology research [138]. Its popularity is not surprising as it enables researchers quantify personality across five distinct traits - Openness, Conscientiousness, Extraversion, Agreeableness and Neuroticism (OCEAN) - using a single personality test.

In the context of conformity literature, the effects of the Big-five personality traits have been investigated primarily in CMC-based studies. For example, Packer [130] analysed effects of openness and conscientiousness traits on conformity behaviour among undergraduate students in an opinion-based experimental task. More specifically, the students were required to indicate their personal opinion on the use of alcohol on campus premises in an online chat room, after being exposed to pro-alcohol use attitudes supposedly coming from their fellow students. The authors found that students with higher conscientiousness and openness were more likely to disagree with the supposed pro-alcohol community stance and publicly express their concerns regarding alcohol use on campus.

We further note that concise versions of the Big-five personality test have been derived to allow for time-efficient personality measurement. For example, a study by Perfumi et al. [134] reported using the Five Factor Adjective Short Test (5-FasT) derived by Giannini et al. [62] based on the Big-five personality traits, to measure effects of personality on conformity behaviour. This inventory consists of 26 dichotomous (true-false) items, in comparison to the 44, 5-point scale (strongly agree-strongly disagree) items in the original inventory. The findings from this study indicate inverse relationships between conformity and three personality traits - neuroticism, extraversion, and agreeableness and a positive relationship between conformity and openness. No effects were observed from conscientiousness.

Therefore, despite the popularity of the Big-five personality traits in Social Psychology, its effects are yet to be fully understood in conformity literature. Existing findings indicate that effects of these personality traits may vary across different experimental tasks and the version of the inventory that is used for measurement. Therefore, in Chapter 4 we investigate effects of the Big-five personality traits on conformity behaviour of participants who complete an online group quiz that contains both objective and subjective questions. We use John and Srivastava's 44-item Big-five personality test [84] to measure user personality, to ensure comparison with prior work. 


\subsection{Summary}

Social conformity behaviour is a function of its contextual and personal determinants. In this chapter, we provide an overview of the literature that has investigated social conformity with regard to these determinants in both physical and CMC groups. We introduce the social conformity terminology that we refer to in the rest of this thesis, describe how contextual and personal determinants of social conformity have been manipulated in prior conformity studies, and discuss reported effects of these determinants on user conformity in physical and CMC groups. By doing so, we highlight research gaps between physical and $\mathrm{CMC}$ conformity literature, and position the original contributions of this thesis - presented in Chapters 4, 5, 6, 7 and 8 - highlighting how each chapter builds up on prior work. Furthermore, findings of prior work also informed the overall experimental methodology of this thesis, which we discuss next in Chapter 3. 


\section{Chapter 3}

\section{Methodology}

This chapter outlines the methodological decisions that informed the studies presented in this thesis. In Section 3.1, we clarify certain fundamental methodological concepts relevant to our work - including how we characterised conformity behaviour, and used different experimental paradigms to artificially induce social pressure situations essential for conformity studies [11,39]. We then describe the overall research process that spanned across the five research studies presented in this thesis, to systematically investigate the research questions presented in Chapter 1 . We also provide details of the quantitative and qualitative data collection techniques used, and motivate their applicability to relevant study goals, in Section 3.2. Additionally, we describe how the presented experiments adhered to ethical considerations in Section 3.3, and provide a general overview of the quantitative and qualitative data collected and how they were analysed in Section 3.4. For additional methodological details specific to the individual studies, refer to the publications attached in Chapters 4, 5, 6, $7 \& 8$.

\subsection{Research Approach}

In this thesis we identify conformity behaviour as an adjustment in user's initial response to the experimental task, after being exposed to group's feedback. Therefore, all studies presented in this thesis follow three main steps to capture user conformity. First, a user is asked to indicate their initial response to the task at hand, without any knowledge of group's responses. Next, they are shown how "others" responded to the same task (group feedback), after which they are asked to indicate their response again.

Moreover, conformity behaviour is analysed only when the user's initial response is opposed by the group's majority in the displayed "group feedback". The response supported by the "majority" as shown in the group feedback is determined in each experiment based on the results of a pilot study, where a similar sample of users complete the same experimental task independently (without any knowledge of group responses). A reasonable incorrect position for the majority to support is chosen based on the popularity of the control group's responses, to ensure that the majority's response is believable. This is especially crucial in the subjective questions, where there is no specific correct response for the opposing majority to go against.

We further emphasise that "group feedback" is created by either using confederates who acted as real participants (Asch's paradigm) or using computer-simulations (Crutchfield's paradigm), based on individual study requirements. More specifically, we used Crutchfield's paradigm to simulate group feedback (in Articles I, III \& V), to avoid exposing users to personal cues of "peers" that can result in confounding effects (in Article I), to control the personal attributes of "peers" that are exposed to users when 
investigating effects of specific personal determinants on user conformity behaviour (in Article III), and also when the remote, asynchronous nature of the study design demanded it (in Article V). Conversely, we used Asch's paradigm in Articles II \& IV - that present studies conducted in an Instant Messaging platform and an online group setting that allowed text-based chatting between group members respectively - to ensure naturalistic communication and interactivity between group members.

The studies presented in this thesis are based on different online group settings - i.e., an online MCQ group quiz (in Articles I, III, IV), an Instant Messaging (IM) platform (Article II), and a Facebook news feed (Article V) - where users were shown group feedback and/or communicated with other group members through computer-mediated means. We used an online MCQ group quiz as the computer-mediated communication medium in Articles I, III, IV because it allowed us to conveniently manipulate the social conformity determinants of interest in each experiment under laboratory conditions (e.g., gender group composition in Article III vs. level of social presence in Article IV), while also exposing users to a typical social pressure situation. Additionally, we also tested for the effects of unidirectional (in Articles I and III) and bidirectional communication (in Article IV) among group members by introducing a text-based chat (no user cues exposed) for group discussion in the latter.

Moreover, we also investigated social conformity in realistic, remote group environments by using an IM platform (Slack) in Article II and by simulating a Facebook news feed in Article $\mathrm{V}$ - thereby also testing conformity effects in both synchronous and asynchronous CMC group settings respectively. In other words, these studies not only diversified the range of CMC platforms used in this thesis to ensure the generalisability of our findings, but also investigated conformity effects in asynchronous CMC mediums (e.g. a Facebook news feed) where the concept of a "group" was less profound. We elaborate further on the study design and the experimental setup used in each of these studies at length, in the corresponding chapters.

Furthermore, the overall research approach that we undertake is purposefully designed to systematically and incrementally investigate the research questions of this thesis across multiple research studies. In other words, we repeatedly investigate effects of certain foundational conformity determinants i.e., majority-minority group size, self-confidence, and user gender - that have shown strong effects in prior conformity literature $[47,139,142,188]$, on top of which we incrementally examine effects of determinants such as age and social presence. In brief, Article I investigates potential effects of a broad range of face-to-face contextual and personal determinants, in an online group setting (RQ $1 \&$ RQ 2), and thus lays the foundation for subsequent studies. Articles II \& III then focus on the effects of age and gender stereotypes on online conformity as they are often reported in online groups (RQ $2 \& \mathbf{R Q} 2(\mathrm{a})$ ), and propose design guidelines to mitigate adverse effects of stereotypical conformity behaviour in online group settings (RQ 4). Article III also expands on negative outcomes of genderstereotypical conformity behaviour (RQ 3). Next, we investigate effects of online social presence in Article IV - as a conformity determinant that is especially relevant in online user groups - and indicate how online social presence can be effectively controlled through online platform design to regulate social conformity behaviour - alongside other contextual and personal determinants (RQ 1, RQ 2 \& RQ 4). Finally, in Article V we 
show how conformity influences can be leveraged in social media platforms to encourage prosocial behaviour in how users infer trustworthiness of online news articles and respond to them (RQ 1, RQ 2 \& RQ 3).

\subsection{User Studies and Participants}

In this section we describe how we align the study setup and participant recruitment to suit specific experimental objectives of the studies presented in this thesis - which includes three laboratory studies (Articles I, III, \& IV), and two remote user studies (Article II \& Article V).

\subsubsection{Laboratory User Studies}

We used laboratory user studies in Articles I, III \& IV to test effects of specific conformity determinants in a reasonably controlled environment (no disruptions). The experimental task was an online MCQ group quiz that participants supposedly complete with other people. As the task closely resonates with online quizzes students often undertake, we recruited participants primarily from within our university. This included both undergraduate and graduate students, coming from different educational backgrounds, with reasonable computer literacy, and proficient in the English language. We recruited an equal number of participants who identify as men or women, to account for possible gender differences in user conformity behaviour.

We followed a common procedure for all the laboratory user studies. Each experimental session included one participant. First, we welcomed the participant and provided them with a Plain Language Statement (PLS) which described the objectives of the study, what participants are required to do during the study, what data will be collected, and what research benefits we expect from the experiment. After giving ample time for participants to read the PLS, a researcher briefly described that the experiment aims to "investigate how online group feedback can impact individual performance in online quizzes" and that participants will be grouped with several others to complete an online group quiz. We purposefully kept the study description vague, as a conformity study's true objectives can not be disclosed to the participants [165]. We then gave participants the chance to ask any questions they had regarding the experiment, and obtained their written consent to participate in the experiment. Participants completed the rest of the experiment in a laboratory room, isolated from others. They were led to believe that their "peers" are also seated in different rooms of the laboratory, and will complete the online quiz simultaneously.

The MCQ quiz was conducted fully online. Before initiating the quiz, a custom-made chatbot assisted participants to familiarise themselves with the online quizzing platform, and showed them how to answer the quiz questions step-by-step. We purposefully used a chatbot to minimise potential experimenter effects $[133,178]$ and confounds that can arise as a result of participants perceiving social presence due to the presence of an experimenter [153], by limiting experimenter-participant interactions during the study. Once the quiz started, participants used the online quizzing platform to provide their 
response to a series of quiz questions - both before and after viewing "group responses" to these questions.

The experimental task and the "group responses" shown to participants changed based on the objectives of the study. In Article I, we used both objective and subjective quiz questions (which are based on general knowledge and high school debating topics respectively) to test the effect of task objectivity on online social conformity. Additionally we used computer-simulated bar charts that showed the percentage of others supporting each answer option to the quiz question (e.g. 90\% supports option "A" and 10\% supports option "B") to indicate the distribution of majority-minority group responses, without exposing any personal cues of supposed "peers" that can result in confounding effects.

Alternatively, in Article III we used objective, gender-stereotypical quiz questions to examine how gender-stereotypical conformity impacts accuracy of user answers to quiz questions. We followed an approach inspired by prior work [101, 102, 105] to determine an appropriate set of quiz questions that can elicit gender-stereotypical perceptions in the community considered in this experiment. We also used stereotypically gendered names and silhouette avatars (as two separate experimental conditions) to represent the number of men and women supporting the majority and minority answers. This decision enabled us to expose participants to majorities and minorities with different gender group compositions, and compare effects of two distinct gender cues, while also ensuring that no cues other than the gender of supposed peers are revealed to participants.

In Article IV we used both objective and subjective quiz questions, to test if the level of perceived social presence moderates effects of task objectivity. We manipulated social presence across three aspects: user representation (by using generic vs. user-specific avatars to represent "peers"), interactivity (allowing participants to discuss group answers with their "peers", or not) and response visibility (final responses publicly visible to the group, or not).

Furthermore, after completing the user study, participants were invited to take part in a semi-structured interview, where we inquired about their experience during the quiz. The interview questions were primarily structured around whether participants felt compelled to conform, and if so - what factors encouraged such behaviour. Our intention was to understand when and why people conform in online group settings from the perspective of the users, to support the interpretation of quantitative data gathered from the user studies. We also encouraged participants to clarify any concerns regarding the experiment before concluding the session.

\subsubsection{Remote User Studies}

We used remote user studies in Articles II and V to test conformity effects in more naturalistic environments, and recruit study participants from the demographics relevant to specific study objectives. For example, Article II presents a study that deploys a MCQ group quiz that consists of age-stereotypical, objective questions through an IM platform $\left(\right.$ Slack $\left.^{1}\right)$. As the study aims to quantify effects of user age and related stereotypes on user conformity, we recruited young and middle-aged adults born in Generations Z and

\footnotetext{
${ }^{1}$ https://www.slack.com
} 
X to participate in the study. Hence, the remote nature of the study helped us recruit participants from the relevant demographics conveniently (outside the university), while also mimicking a naturalistic online group setting. We also determined a suitable set of quiz questions to trigger age-stereotypical perceptions in the age groups considered, using an approach inspired by prior work [101, 102, 105]. Similarly, in Article V we investigate the occurrence and outcomes of conformity behaviour in how people perceive and respond to real and fake news articles posted on Facebook. As we used a set of articles based on news related to the US, we deployed our study on Amazon Mechanical Turk $^{2}$ to recruit English speaking, US residents who are registered users of Facebook and thus are more likely to be exposed to said articles.

As with the laboratory user studies, we took steps to inform participants about the study objectives, procedure and potential benefits, and obtained their consent before initiating the experiment. In Article II, we did so through email, whereas in Article V we provided interested crowdworkers with the Plain Language Statement through the Amazon Mechanical Turk interface where they could indicate their consent electronically.

Moreover, due to the synchronous nature of the study presented in Article II, we used a chatbot to train and support participants as they progress through the quiz in their groups. The presence of a bot was especially important in this study, given its remote nature and the absence of a human experimenter for support. Conversely, as the study presented in Article V was deployed as an asynchronous survey that required participants to indicate their perceived trustworthiness of a set of news articles, both before and after reading comments posted by others (i.e. group feedback), a chatbot was not necessary.

Moreover, due to the remote nature of the user study presented in Article II, we chose to collect qualitative data regarding participants' experiences during the study i.e. when and why they conformed during the experiment - through a brief post-test survey to which participants provided textual answers. We chose not to collect qualitative data from the crowdsourced study, because the objective was to quantify conformity influences on user behaviours in online settings and not to understand its dynamics - as in prior studies.

\subsection{Ethical Considerations}

We took several steps to ensure appropriate ethical conduct of the presented research studies - which was especially crucial given the limited disclosure of the study's objectives to participants in the beginning and the use of deception to generate social pressure situations (through confederates and simulated peer responses) as expected in social conformity research [165]. The experimental procedures followed in the studies presented in this thesis, received ethical clearance from the University of Melbourne Human Ethics Advisory Group - well before data collection was initiated. Next, we describe the steps taken before, during and after the experiments to ensure their ethical conduct.

We understand that being subjected to opposing group opinions - especially when they do not accurately represent reality - can be stressful to participants of conformity

\footnotetext{
${ }^{2}$ https://www.mturk.com
} 
studies [11]. Hence, in Articles I and IV where we included subjective, opinion-based tasks in the quiz, we avoided using overly sensitive topics that might bring discomfort to potential participants. The subjective topics used for these experiments were extracted from a list of high school debating topics published on ThoughtCo ${ }^{3}$, that the research team considered appropriate for use with regard to potential participants.

Moreover, before initiating the quiz, participants were given ample time to read through a Plain Language Statement that explained a given study's objectives, the data that will be collected, how the collected data will be stored and used for research, and potential benefits of the study to the overall research community. We emphasised that their participation is voluntary, and that they have the option to withdraw their participation from the experiment at any time during or after the study - without consequences. Subsequently, we obtained their consent through a paper-based or an electronic consent form, prior to starting the experiment.

Furthermore, debriefing participants after a conformity study is essential to explain the use of limited disclosure and deception [11]. Therefore, in the laboratory user studies we debriefed all participants individually, upon completion of the semi-structured interview, to clarify the real objective of the experiment (i.e. understanding the dynamics of social conformity influences in online groups). We explained that limited disclosure was necessary to ensure that they behave naturally during the experiment to collect reliable and valid data with regard to user conformity behaviour. We also explained that the "group responses" shown to them during the experiment were either computer-simulated or generated by confederates who were instructed to do so based on a script - which allowed us to expose all participants to consistent social pressure situations. Subsequently, we asked participants if they wish to withdraw their data from the experiment - but none chose to do so. Similarly, we also explained the use of limited disclosure and deception to participants at the end of each remote study session using the chatbot in Article II and through an electronic document outlining the above details in the study presented in Article V. Remote participants also had the opportunity to reach out to the research team through email to request further information and withdraw their data from the experiment.

Before data analysis, we anonymised the data collected so that they can not be linked to a specific participant. We did not collect or store identifiable information of participants - other than demographics relevant to specific study objectives (e.g., gender, age). The user data from the conformity experiments and audio files from the interviews were stored in a firewall-protected secure server, that could only be accessed by authorised users using a secure password that is changed every six months.

Furthermore, we ensured that all participants were fairly compensated for the time spent completing the experiment. For each study, this was determined based on the average time taken by pilot participants to satisfactorily complete the experimental task, and the minimum hourly wage at the time, of the country (Australia or the USA) from which participants were recruited. In Article $\mathrm{V}$ where crowdworkers were recruited, we included Gold Standard questions $[30,44]$ to determine whether participants were attentive during the study, and did not reject their submissions unreasonably.

${ }^{3}$ https://www.thoughtco.com/debate-topics-for-high-school-8252 


\subsection{Data Analysis}

We used a mixed-method method study design in Articles I, II, III \& IV in order to quantify the effects of analysed conformity determinants on user conformity behaviour, while also using qualitative data to understand how these social conformity influences were perceived by participants.

\subsubsection{Quantitative Analysis}

The quantitative data collected through the user studies primarily consists of initial and final responses of participants to the experimental tasks, and their self-reported confidence ratings on the selected (initial and final) responses. In Articles I, II, III \& IV, participant responses were in the form of answers to quiz questions, whereas in Article $\mathrm{V}$ they were trustworthiness ratings indicated by participants to several news articles. In all the studies presented, we repeatedly tested for conformity behaviour in all participants, by asking them to complete a series of tasks during the experiment (e.g., a series of MCQ questions). Furthermore, we defined conformity behaviour as adjusting one's initial answer (in Articles I, II, III \& IV) or trustworthiness rating (in Article V), to align with the majority's response on the same task. In other words, conformity was considered as a binary outcome variable (i.e. conformed or not).

We used statistical modelling to analyse the collected quantitative data with regard to user conformity/non-conformity behaviour. More specifically, we used Generalised Linear Mixed-effects Models (GLMM) to quantify potential fixed (main and/or interaction) effects from the contextual and personal determinants manipulated during an experiment, on the binary outcome variable conformity (that followed a non-normal distribution) - while also accounting for random effects that can rise from individual variations in participants. We did so by specifying participant (or the participant ID) as a random effect in the GLMM, which enabled us to use the model to support broader inferences about the larger population of participants. Furthermore, using GLMMs allowed us to simultaneously consider many variables that could potentially impact participants' conformity behaviour. For instance, Articles I, III, IV \& V examined effects of over ten predictor variables on participant conformity using GLMMs. Moreover, following research standards in HumanComputer Interaction [100], we quantified statistical significance and rejected the null hypothesis using a confidence level of $95 \%$ (i.e. with a p-value less than or equal to 0.05 ).

\subsubsection{Qualitative Analysis}

The qualitative data presented in Articles I, III \& IV are from semi-structured interviews, whereas in Article II we present data coming from participant responses to a posttest survey. We used semi-structured interviews primarily to determine "how" and "why" conformity determinants manipulated during a specific experiment impacted user conformity behaviour [14]. Semi-structured interviews have been widely used in similar experimental setups in Human-Computer Interaction research, where the range of user experiences is unknown and hence need to be analysed from a broader point of view but with redirection when relevant $[79,127,149]$. Similarly, we used a mix of closed and 
open questions in the post-test survey in Article II, to allow participants to explain their conforming and/or non-conforming behaviour during the remote user study - which is one reason why qualitative surveys are deployed in Human-Computer Interaction research alongside other quantitative measurements [80, 128, 129].

The interview data were audio-recorded and later transcribed on to online spreadsheets by 2-3 people from the research team. While transcribing, we anonymised the interview data and referred to participants using unique participant IDs assigned to them during the experiment (e.g. P1). In Articles II \& III we retained age and gender details of the participants, as they were relevant to the research objectives of these studies and provided context for subsequent analysis and presentation of the qualitative data.

We used thematic analysis [25] - a popular qualitative analysis technique widely used in Human-Computer Interaction research $[2,21]$ as well as in Psychology [26] to make sense of people's behaviours - to collaboratively analyse the collected qualitative data. More specifically, we conducted an inductive thematic analysis in Articles I \& II, where we derived themes from the qualitative data itself, because the literature was insufficient to develop a framework or a codebook to analyse qualitative data related to effects of contextual and personal determinants, and age-stereotypes on online conformity behaviour. Hence, during this process we identified emerging themes from the data and then collaboratively finalised the themes to be discussed in each article based on their relevance to the research objectives of a specific study.

Conversely, we used a deductive thematic analysis approach in Articles III \& IV to link data with a set of predetermined themes that focused on the research questions of the specific experiment. For instance, the qualitative analysis in Articles III \& IV focused on the effects of contextual and personal conformity determinants - informed by our findings in Articles I \& II. Additionally, we also formulated themes around gender-stereotypes in Article III, and the three aspects of social presence investigated in Article IV.

\subsection{Limitations}

Although the studies presented in Articles I, II, III, IV \& V were meticulously designed and conducted, we acknowledge several limitations that we describe next.

We used a mix of both laboratory and remote user studies to quantify effects of several conformity determinants in different asynchronous and synchronous online group settings (e.g., online quizzes, IM, social media). However, we acknowledge that our societal interactions take place in a vast variety of online group settings that were not investigated in this thesis. Additionally, the information revealed to participants in the study setups used were carefully controlled to quantify effects of relevant determinants, and avoid confounding effects from variables irrelevant to study objectives. For instance, in Article III we purposefully chose user cues that only revealed gender of supposed peers, as the intention was to quantify effects of gender and related stereotypes on online conformity. But in reality, online user representations such as photographs can simultaneously reveal other user cues (e.g., age, race) that can also contribute towards stereotypical behaviour in online settings $[23,27]$. Hence, further work is necessary to 
determine how findings of this thesis manifest in other types of uncontrolled, real-world online group settings.

Furthermore, participants were exposed to artificially induced social pressure conditions that may not represent reality. In other words, participants saw "group feedback" that was either computer-simulated or generated using confederates - and hence did not occur naturally. However, this decision was necessary to ensure that all participants were exposed to the same social pressure situations in a given experiment, and is supported by prior work in both physical [11,39] and online [19, 139] conformity literature. We have justified our decision to use confederates or simulations to mimic group feedback in the attached publications. Future work should explore and quantify social conformity behaviour in online group settings where "group feedback" is allowed to manifest organically (e.g. online debating platforms).

Additionally, while our participants came from different educational backgrounds and levels, they demonstrated above average digital literacy - which may have reduced their susceptibility to online social conformity influences. Thus, further work may be required to determine whether our observations can be generalised to a wider population.

We also note that the findings reported in Articles II \& III with regard to effects of age and gender stereotypes on online conformity, are specific to participants who are young and middle-aged adults (in Article II), and identify as either a man or a woman (in Article III). Furthermore, as perceptions of age and gender may vary cross-culturally, more work is required to determine how our findings replicate across other age groups, gender identities and cultures. For instance, the study presented in Article III is based on a traditional gender binary model, whereas in reality what it means to be gendered varies from place to place, in different times, and is heavily influenced by race, age and other factors [88]. Additionally, the stereotypical experimental tasks used in Articles II \& III were carefully chosen to insinuate age and gender stereotypical perceptions in participants who primarily came from WEIRD communities [85]. Thus, the same set of questions may not generate similar user behaviour cross-culturally.

Furthermore, we acknowledge that social conformity is only one of many forms of social influence that impact our online behaviours. However, as the studies presented in this thesis were carefully designed to specifically investigate the dynamics of social conformity in online group settings (in terms of its determinants and outcomes) and determine how future online group settings can be designed to account for conformity influences, they do not account for potential effects from other forms of social influences. Therefore, while a discussion on how social conformity may interact with other forms of social influences in online settings is an interesting avenue for future work, it is not within the scope of this thesis.

\subsection{Summary}

This chapter outlines the overall research methodology followed in this thesis. In brief, we used five meticulously designed laboratory and remote user studies to systematically investigate the research questions identified in Chapter 1. These studies collected and analysed quantitative and qualitative data on how users perceived and responded to 
social pressure situations in different online group settings. Next, we present five research articles published at leading peer-reviewed Human-Computer Interaction research venues, that further elaborate on the research design, execution and findings of these studies in Chapters 4, 5, 6, $7 \& 8$. 


\section{Chapter 4}

\section{Contextual \& Personal Determinants of Online Social Conformity}

\subsection{Introduction}

Literature has primarily focused on investigating social conformity in physical groups, specifically focusing on its different contextual [11, 20,42, 82] and personal determinants [39, 47, 91, 142]. While increasing attention has been given to conformity behaviour in CMC groups in recent years (see Table 2.1), current research is insufficient to fully understand the impact of this powerful social influence in online group settings. Additionally, while a few studies have examined independent effects of certain contextual and personal conformity determinants in CMC groups [97, 139], we argue that investigating the collective impact of these determinants is crucial to better understand their true effects.

In this chapter, we investigate social conformity as a function of its contextual and personal determinants in an online group setting. We quantify how several wellestablished contextual (i.e., majority-minority group sizes, number of minorities, task objectivity) and personal (i.e., user gender, self-confidence, big-five personality traits) determinants of face-to-face conformity behaviour, impact conformity behaviour of participants who complete an online group quiz. Our results indicate that users are more likely to conform in objective quiz questions, when opposed by larger than smaller majorities, and when unsure of their personal answer. Additionally, those with higher conscientiousness and neurotic personality traits tend to conform more often than others. No significant effects were observed from the number and the size of the minority groups, and user gender. Thus, our findings show how the effects of popular contextual and personal determinants of physical conformity replicate in CMC groups, and emphasise that conformity should be investigated and better understood in terms of both its contextual and personal determinants.

We provide more details of the study in the attached publication, Article I. The results of this paper also inform the research focus of our subsequent studies (i.e., what determinants to investigate further), which we describe in the relevant chapters. 


\subsection{Article I}

Copyright is held by Elsevier Ltd. This is the authors' version of the work. It is posted here for your personal use. Not for redistribution. The definitive Version of Record was published in:

Wijenayake, S., van Berkel, N., Kostakos, V., Goncalves, J. (2020). Impact of Contextual and Personal Determinants on Online Social Conformity. Computers in Human Behavior, 108, 106302. https://doi.org/10.1016/j.chb.2020.106302.

Ethics ID: 1853038, The University of Melbourne Human Ethics Advisory Group. 


\title{
Impact of Contextual and Personal Determinants on Online Social Conformity
}

\begin{abstract}
Despite decades of research concerning social conformity and its effects on face-to-face groups, it is yet to be comprehensively investigated in online contexts. In our work, we investigate the impact of contextual determinants (such as majority group size, the number of opposing minorities and their sizes, and the nature of the task) and personal determinants (such as self-confidence, personality and gender) on online social conformity. In order to achieve this, we deployed an online quiz with subjective and objective multiple-choice questions. For each question, participants provided their answer and self-reported confidence. Following this, they were shown a fabricated bar chart that positioned the participant either in the majority or minority, presenting the distribution of group answers across different answer options. Each question tested a unique group distribution in terms of the number of minorities against the majority and their corresponding group sizes. Subsequently, participants were given the opportunity to change their answer and reported confidence. Upon completing the quiz, participants undertook a personality test and participated in a semi-structured interview. Our results show that $78 \%$ of the participants conformed to the majority's answers at least once during the quiz. Further analysis reveals that the tendency to conform was significantly higher for objective questions, especially when a participant was unsure of their answer and faced an opposing majority with a significant size. While we saw no significant gender differences in conformity, participants with higher conscientiousness and neuroticism tended to conform more frequently than others. We conclude that online social conformity is a function of majority size, nature of the task, self-confidence and certain personality traits.
\end{abstract}

Keywords: Online social conformity, Majority size, Task type, Self-reported confidence, Personality traits, Minority groups. 


\section{Introduction}

Conformity is a powerful social phenomenon that encourages individuals to change their personal opinions and behaviour to agree with an opposing majority (i.e. the greater proportion of the group members with a contradicting opinion or behaviour) [1]. Such behaviour is predominantly visible as we tend to fit in to our social groups, to be 'liked' and to be 'right' [2]. In other words, social conformity can lead to people not expressing their own judgements and opinions when facing peer pressure in groups, which could be detrimental to the effectiveness of groups in decision making and innovative thinking [3].

This psychological mechanism has been widely studied with regard to face-to-face groups, specifically focusing on its diverse contextual and personal determinants. For example, it was observed that when placed in a group setting, the likelihood of an individual conforming to the majority was influenced by various contextual factors such as the size of the majority group $[4,5]$ and the nature and difficulty of the task at hand (i.e. objective tasks with one correct answer or subjective tasks where the answer is based on or influenced by personal feelings, tastes, or opinions) [6,7]. Moreover, literature suggests that personal factors such as participant gender [8], self-confidence [9] and personality [10] may also impact susceptibility to social conformity differently.

However, it is unclear to what extent observations resulting from these seminal studies apply to online settings. This is of particularly importance as our social interactions increasingly shift to diverse online paradigms such as discussion forums, social media, polls and learning platforms $[11,12]$. As such online groups are inherently dissimilar to face-to-face groups in terms of anonymity and reduced social presence [13], their susceptibility to social conformity is likely to vary. While existing literature provide some evidence for the presence of conformity in computer-mediated settings $[14,15,16,17]$, and evaluate the effects of several aforementioned factors independently [18, 19, 20], they fail to assess the combined effects of such determinants. We argue that understanding the collective impact of such determinants could better explain their relative importance while also rationalising conformity behaviour. Thus, we extend the existing literature by thoroughly exploring possible direct and combined effects of contextual and personal determinants of conformity in anonymous online settings. While online settings differ from face-to-face settings in aspects beyond anonymity (e.g. social presence), we do not investigate aspects of online social interactions beyond anonymity in the current study.

To explore online social conformity as a function of contextual and personal determinants, we deployed an online quiz with multiple-choice questions (MCQs) of objective and subjective nature. Participants first answered each question privately while denoting their self-reported confidence on the selected answer. Next, our software displayed the distribution of votes across the different answer options of the MCQ, as chosen by other participants. Participants were then given the opportunity to change their initial answer and self-reported confidence. We also collected big-five personality test scores (where personality is identified in terms or openness, conscientiousness, extraversion, agreeableness, and neuroticism) [21], to assess the personality of each participant towards the end of the quiz. Through our study we investigate the following research questions with regard to online social conformity: 
RQ1: How do contextual determinants like majority and minority group sizes, number of minorities present and nature of the task impact the likelihood of an individual conforming to the majority's judgement in an online setting?

RQ2: How do personal determinants like gender, self-confidence and personality impact the likelihood of an individual conforming to the majority's judgement in an online setting?

\section{Related Work}

\subsection{Conformity in Face-to-Face Groups}

Social conformity was first explored in physical face-to-face groups. Asch' conformity experiments $[1,22]$ were pivotal among early research on social conformity, where an astonishing $33.3 \%$ of the participants conformed to a clearly incorrect yet unanimous majority, in a simple line matching task, confirming that individual judgements can be swayed under pressure. A subsequent study by Deutsch and Gerard [2] rationalised conformity behaviour as having 'normative' and 'informational' influences. The authors described 'normative influence' as the tendency to conform to expectations of the majority to be 'liked' within the group. Accepting the majority's judgement to be more accurate than one's own judgement (especially in ambiguous situations) was explained as 'informational influence'. The impact of 'normative influences' was further confirmed by more recent work where conformity was seen as an outcome of individuals' desire to fit in with the group [23] and ensure a sense of belonging [24]. Moreover, Levine [23] emphasised the effects of 'informational influence' on conformity, where individuals turn to groups for guidance in ambiguous situations where the 'correct' response is unclear.

Further studies on face-to-face social conformity have primarily focused on identifying contextual and personal determinants of conformity. Literature reveals that rates of conformity vary based on contextual factors such as the majority group size $[4,5,25]$ and the nature of the task $[7,6]$. In addition to contextual determinants, personal factors such as gender [8, 26, 27], self-confidence [9, 28, 29, 30] and an individual's anxiety levels [31] have been identified as important determinants of social conformity.

Despite the extensive literature on social conformity in face-to-face groups, this form of social influence is currently underexplored for computer-mediated online groups. As human interactions increasingly shift towards online platforms [11, 12], whether and how social conformity manifests in virtual groups is of interest to the research community. Next, we review previous work on conformity in online settings, and identify the gaps in the literature that we aim to address in our work.

\subsection{Conformity in Online Settings}

Due to the rapid advancements of the Internet, individuals are actively interacting with each other through diverse online platforms (e.g., discussion forums, support groups, learning platforms) to satisfy their informational and social requirements $[11,12]$. Thus, one can argue that social influences affecting people in face-to-face groups may also manifest in online settings. 
Cinnirella \& Green [17] explored the susceptibility of individuals to 'normative influence' in computer-mediated groups. The study extended Asch's line experiment by allowing participants to select their answers through computer-mediated communication (a personal computer), offering them anonymity. Similar to Asch's experiment, the participants saw a majority (consisting of confederates of the researcher) providing a uniformly incorrect response, before they gave their personal answer. The results of this experiment were compared against a traditional face-to-face situation in which participants answered the same test in physical groups. The study concluded that while conformity was significantly reduced in the anonymous computer-mediated group condition when compared to the face-to-face condition, the unidirectional feedback from an anonymous and contradicting majority was sufficient to trigger conformity behaviour. Similar findings were put forward by Smilowitz et al. [32], confirming the above conclusion.

Despite being criticised for its negative impact on group decision making and productivity [2], literature displays both positive and negative implications of social conformity in online settings. For example, work by Sukumaran et al. [15] investigated how social conformity may encourage adapting to acceptable standards and structure within online communities. The study was conducted on an online news website, where participants saw a set of 'high-thoughtful' or 'low-thoughtful' comments added by prior users for a news item, before they were asked to post their own comments. The results of this study emphasised that 'high-thoughtful' comments added by prior users motivated subsequent participants to contribute with similar or additional effort, even when there was no other interaction between the participants and the previous users. The study confirmed the existence of 'normative influence' and social conformity, and its applicability in shaping the amount of effort users put into their contributions in online communities.

However, a more recent study by Beran et al. [14] exploring conformity among graduate students in a virtual learning environment revealed contradicting observations. This study deployed an online quiz on curriculum-based tasks, where a proportion of the participants were shown incorrect peer answers prior to answering the questions, while the others attempted the quiz independently. Authors observed that a significant proportion of students conformed to the incorrect responses of their peers, despite the static and unidirectional nature of the peer feedback. Students rationalised their conformity behaviour as an outcome of self-doubt and lack of knowledge on the tested content, emphasising the effect of 'informational influence' on conformity. More interestingly, students who were shown peer answers were seen to obtain fewer correct answers than the students who completed the quiz by themselves.

Furthermore a study by Sharma et al. [16] explained how individuals seeking support from online support groups were encouraged to conform to accepted group norms of communication, to receive better support. Individuals who conformed to the group's linguistic norms received more positive support and feedback than the ones who did not. On one hand, conforming to group norms improved the sense of belonging and security within the community, so that sensitive mental health issues could be openly discussed. However, authors also argued that pressure to conform to the group's norms may cause unnecessary distress to individuals seeking support from online communities. 
Having acknowledged that social conformity can have mixed effects in online social groups, we argue that in order to derive positive outcomes through this powerful social influence, a thorough understanding of its determinants is required. While literature on social conformity in face-to-face groups may lay adequate groundwork, it is important to recognise that online groups are inherently dissimilar to face-to-face groups due to anonymity and reduced social presence they provide [13]. Thus, the dynamics and implications of social conformity in online environments could be considerably dissimilar to that of physical faceto-face groups. While we acknowledge that online settings differ from face-to-face settings in aspects beyond anonymity, in this work we thoroughly investigate the effects of contextual and personal determinants of social conformity in an anonymous online setting with static and unidirectional peer feedback.

\subsection{Contextual Determinants of Social Conformity}

Early literature exploring the determinants of social conformity attempted to explain conformity as an outcome of various contextual factors. Among such determinants group size of the influencing source (the majority) and the nature of the task has been prominently researched [22, 5, 33, 34, 1, 2, 35, 36].

Quantifying the effect of majority group size on conformity has been an interest of many researchers and a variety of theories have been put forward. For instance, Asch [22] noted that against a minority of one, the influential power of the majority increased until its third member. Adding a fourth member to the majority did not generate a higher conformity influence. This notion was further established by subsequent experiments on group size and conformity [5, 33]. Moreover, a study by Chester et al. [37] rationalised that larger majorities exert more pressure on individuals to conform as a result of higher 'normative' and 'informational' influences. Latane et al. [34] further expanded this understanding by exploring the incremental impact generated by each additional member of the majority. They observed that while the influential power of the majority increased as the group grew in size, the incremental impact generated by each additional member reduced.

However, the above studies considered unanimous majorities of varying sizes, against a minority of one (the participant). The studies subsequently failed to determine how the majority's group size would affect conformity in the presence of other minorities, which is a more typical situation in real world group settings.

Furthermore, the impact of majority's size on its ability to trigger conformity among individuals is yet to be explored in online settings. However, a study by Lowry et al. [18] observed that computer-mediated communication could reduce typical process losses such as conformity visible in larger groups, as compared to face-to-face communication. Thus, it is plausible that online groups may not be affected by adverse influences of social conformity, even with increasing group sizes. However, this notion is yet to be systematically tested in online settings.

Literature also supports the notion that the likelihood of a person conforming to the majority's judgement varies based on the nature of the task at hand. Early experiments on face-to-face groups $[1,2]$ explored the effects of conformity in objective tasks while Ferguson [35] observed conformity manifesting in tasks of attitudinal nature. Blake et al. [36] 
compared rates of conformity in questions of both subjective and objective nature to observe that participants conform more to majority's opinions on subjective content than on objective content. The authors concluded that the motivation to achieve correct answers to objective questions outweighed the appeal of conforming to an incorrect majority.

A more recent study by Laporte et al. [19] reveals similar observations with regard to task difference in online groups. Even though the rates of conformity were significantly lower than in physical groups, higher conformity was visible as participants answered subjective questions, when compared to questions of objective nature. The authors presume that reduced social presence in online contexts (when compared to face-to-face groups), may reduce the effects of 'normative conformance' but does not completely eliminate its effects.

In this study we expand the existing knowledge on the effects of majority and minority group size, number of minorities and nature of the task, by exploring conformity behaviour among individuals in the presence of a range of majority and minority group distributions for tasks of objective and subjective nature.

\subsection{Personal Determinants of Social Conformity}

Deutsch and Gerard [2] explained that individuals conform to the majority's response when they are unsure of the 'correct' response. In such situations, individuals perceive majority's judgement more likely to be accurate than their own. This implies that confidence in one's personal judgement as well as the judgement of the influencing source, may be important determinants when exploring conformity behaviour. This notion has been investigated in face-to-face groups. For instance, Samelson [28] observed that participants of an estimation task demonstrated higher conformity when they displayed lower confidence on personal answers and higher confidence on majority's answer. Similar observations were made with regard to self-confidence and confidence on partner's answer in face-to-face groups [9, 29, 30].

The impact of confidence on conformity is yet to be explored in detail with regard to online groups. However, previous work has shown that individuals in online groups who presumed the experimental tasks to be difficult, conformed to incorrect majorities more than those who did not [20]. Thus, we argue that similar effects of self-confidence on conformity may be visible even in online groups.

As conformity is applicable to all individuals, why certain individuals are more susceptible to its influence than others is a thought-provoking question. Researchers have attributed such changes in susceptibility to differences in personality and character [10, 38]. More specifically, Meunier and Rule observed that higher test anxiety led to more conformity [31]. While there is a substantial amount of work on the impact of personal factors on online behaviour [39, 40], not many studies were able to establish a clear relationship between personality and conformity, mostly due to the lack of appropriate tools to assess individual personalities. However, since these early studies, more robust personality assessment tools, such as the Big-five inventory [21] have been introduced, which can enable a better understanding of the relationship between personality and conformity in online groups.

Furthermore, gender differences and its impact on group conformity is a well-researched area in literature. Early literature emphasised that women were more susceptible to external influences than men [27]. Thus, women were seen to conform more frequently than men 
under group settings [8]. This observation was explained as an outcome of social roles imposed on individuals such that, men were expected to be task-oriented while women were expected to be cooperative and considerate of the group goals [26]. Expectations to adhere to such gender roles may have contributed to differences in conformity behaviour.

However, literature exploring effects of gender on conformity in computer-mediated groups are inconclusive. While some confirm that women are more likely to conform to the majority's opinion than men [41], more recent studies observe no statistically significant gender differences with regard to conformity behaviour in online settings [20]. Thus, more work is required under this topic.

Based on the cumulative evidence provided by existing literature, we observe that conformity is a function of multiple contextual and personal determinants and thus needs to be explored from a wider perspective in order to truly understand the factors at play.

\section{Method}

We conducted our experiment as an online quiz with multiple-choice questions (MCQ). MCQ quizzes have been widely utilised in many recent studies related to online social conformity $[14,20,19]$. This methodological decision enabled us to control the independent variables (such as group distributions and question types) to suit the requirements of the experiment, while simulating a plausible real world online environment.

Our experiment was approved by the Human Research Ethics Committee at our university. Informed written consent was obtained from each participant prior to data collection. Each participant spent 60 minutes completing the experiment, which included an individual briefing session, training, completing the quiz, and a final face-to-face interview. Participants received a $\$ 15$ gift voucher for participation.

\subsection{The Questions}

The MCQ quiz contained 34 multiple-choice questions, with an equal distribution of subjective and objective questions. The subjective statements were extracted from a list of high school debating topics published on ThoughtCo (www.thoughtco.com). We avoided choosing overly sensitive subjective questions due to ethical concerns as well as the fact that individuals are less likely to change their opinions on such topics. Objective questions included a mix of logic, vocabulary, and general knowledge questions extracted from Mensa International workout (www .mensa.org), Merriam-Webster vocabulary quizzes (www . merriam-webster.com), and Examveda, a well-known general knowledge question repository (www. examveda.com) respectively.

\subsection{Participants and Procedure}

We recruited 50 participants from different educational backgrounds which included Engineering, Science, Arts and Design, Finance and Accounting, Management and Law fields. Participants' age ranged between $18-34$ years (women $=25$, men $=25$ ). All participants were invited to take part in this study via an online notice board. Interested candidates 
were asked to complete a screening questionnaire requesting their gender, level of education, and area of expertise. Researchers then filtered out the required quota of participants representing different gender groups, educational levels, and areas of expertise.

The experiment was conducted in a laboratory with one participant per session, under the supervision of a researcher. Participants were informed that the objective of the experiment was to determine the importance of group feedback in online settings, as the true purpose of the study could not be disclosed prior to the quiz as expected in a conformity study [42].

Participants then completed an online form which collected their gender, age, and educational background. Upon submitting their demographic details, participants were greeted by a conversational agent named 'QuizBot', which assisted them in familiarising themselves with the environment through two training questions as displayed in Fig. 1.

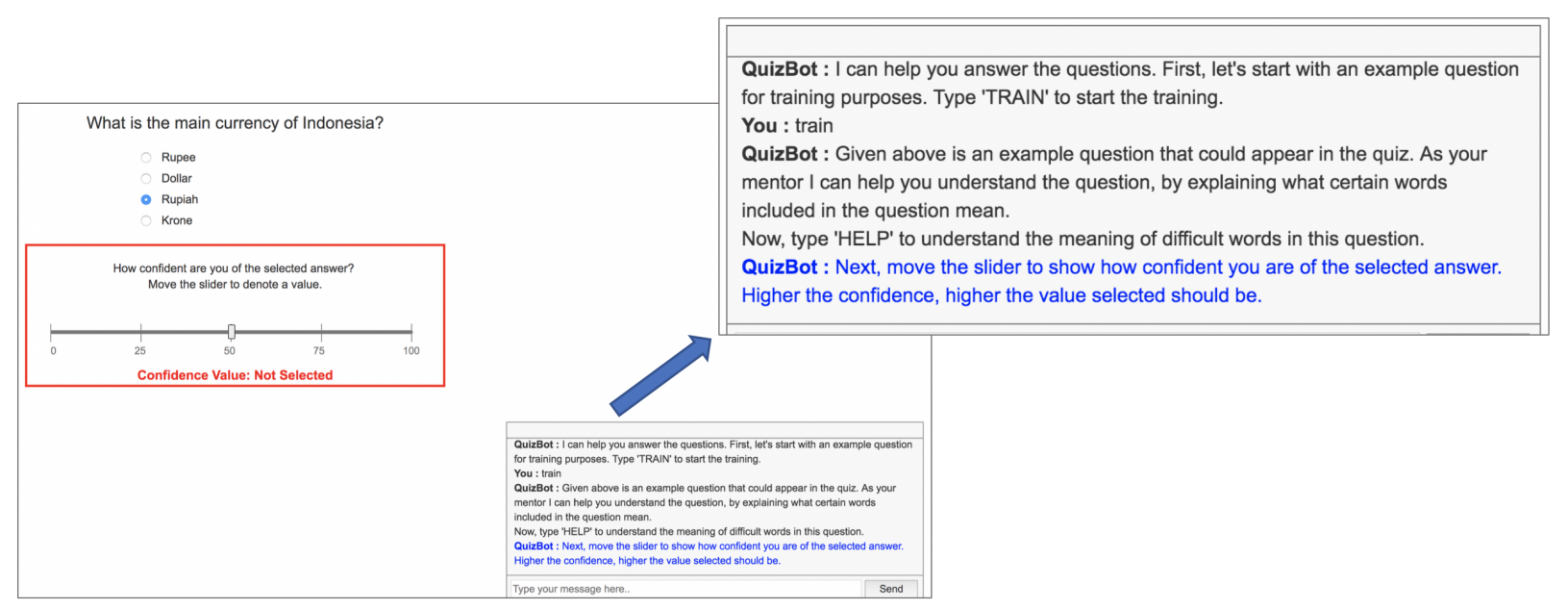

Figure 1: QuizBot assisting the participants with step-by-step instructions.

Training was considered essential in order to ensure that the participants were aware of the process to be followed during the quiz. We utilised the 'QuizBot' to provide stepby-step instructions to participants during the training while minimising the intervention and influence of researchers. This enabled us to simulate a typical online setting where the participants were by themselves.

After training, participants were able to begin the actual quiz. For each question, participants were instructed to select their answer and rate how confident they were with their selection (see Step 1 in Fig. 2). Self-reported confidence levels were denoted using a scale ranging from 0 - 100 with higher values representing higher levels of confidence.

Subsequently, participants were shown a fabricated bar chart as feedback, claiming to represent how their peers answered the same question (see Step 2 in Fig. 2). A similar approach was successfully leveraged in previous work investigating social conformity [20]. We manipulated the bar charts to position the participants either in the majority or the minority, presenting the distribution of votes across the different choices. The majority minority group distributions (such as $90 \%-10 \%, 80 \%-20 \%, 70 \%-20 \%-10 \%$ etc.) were 
tested in a random order, while each group size in a given combination was also randomly adjusted by a value between $1 \%$ and $4 \%$ to ensure their plausibility. For example, in a situation where a $80 \%$ majority and a $20 \%$ minority needs to be displayed to participants, one participant may see a majority of $82 \%$ and a minority of $18 \%$ (adjustment factor $= \pm 2$ ) while another could see a majority of $76 \%$ and a minority of $24 \%$ (adjustment factor $= \pm 4$ ), demonstrated through the feedback charts.

Upon seeing the answers of their peers, participants were given the option to maintain their original answer or make changes to the selected answer option and confidence (see Step 3 in Fig. 2).

Step 1

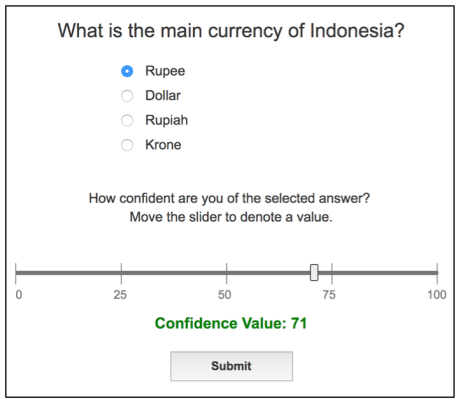

Step 2

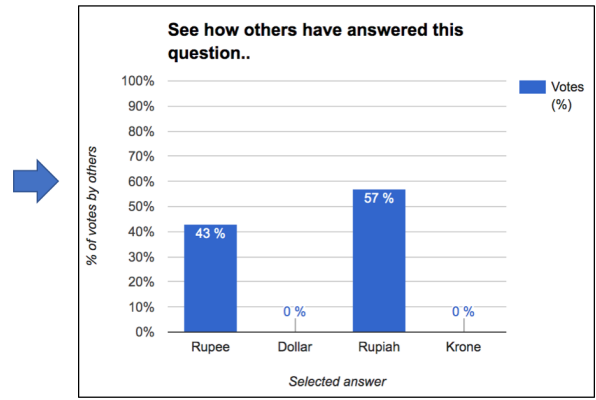

Step 3

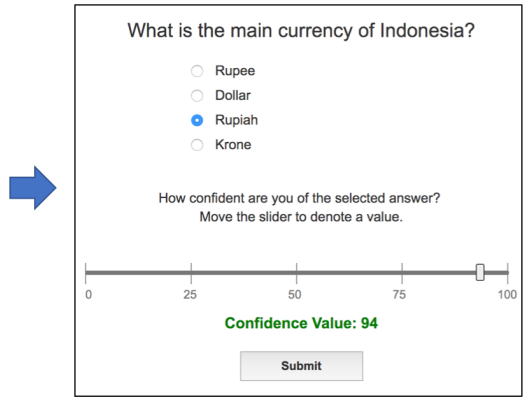

Figure 2: Steps to be followed during the quiz : (1) Initial answer and confidence (2) Feedback (3) Final answer and confidence

Beyond assisting with training, the bot kept track of the progress of the participants while also reminding them about the subsequent steps. Moreover, once the feedback charts were displayed for each question, the bot interpreted the results explaining the group distribution among answer options as to avoid any confusion.

For all question items, we recorded the answer options and confidence levels of participants both before and after viewing peer answers. We also note that all participants answered a mix of subjective and objective questions during the quiz. Furthermore, we counterbalanced the presentation of subjective or objective questions for any given group distribution in order to account for possible interactions between group distributions and question type.

Upon completing the quiz, participants were instructed to undertake a self-assessed Big5 personality test online. The personality test included 44 test items extracted from [21]. Once the personality test was completed, we conducted a semi-structured interview with each participant, in which we debriefed the participants on the true objective of the study. Subsequently, we enquired them about any prior experience facing social pressure in physical or online groups, and whether they felt an urge to change their initial answer during this quiz and why. We were also interested in understanding how participants perceived the group feedback, and the usability and appropriateness of a bot as a training tool (compared to static textual instructions) in online settings. 


\subsection{Pilot}

As the participants were unaware that the feedback they received was fabricated, it was important to decide how the majority and minority groups could be positioned among the four answer options in a credible manner. For example, in a group distribution where the participant would be in the minority of $20 \%$, challenged by a majority of $70 \%$ in the presence of another minority of $10 \%$, it was important to decide which answer options should represent the $70 \%$ majority and the $10 \%$ minority. This placement of majorities and minorities was especially crucial in the subjective questions, where there was no one correct answer.

To address this requirement, we conducted a pilot study with an additional 26 participants (13 women and 13 men). Pilot study participants answered the quiz individually under lab conditions (they were not shown answers of other participants). We observed that for subjective questions results dispersed among at least three answer options. Moreover, for most objective questions a majority selected the correct answer option, while smaller minorities scattered among the other answer options. We arranged the answer options for each question based on the descending order of number of votes it received from the pilot study, to determine a plausible arrangement for the majority and minority groups when fabricating the charts for the main experiment.

\section{Results}

We collected 36 responses from each of the 50 participants (2 training questions and 34 quiz questions). Responses to training questions were removed from the data set prior to analysis, which resulted in 1700 responses. The participants were in a majority in 800 responses and in a minority for in the remaining 900 responses (equally distributed between objective and subjective questions). We highlight that our intention was not to compare results between majority and minority groups, but rather explore the impact of diverse group distributions on conformity behaviour among individuals.

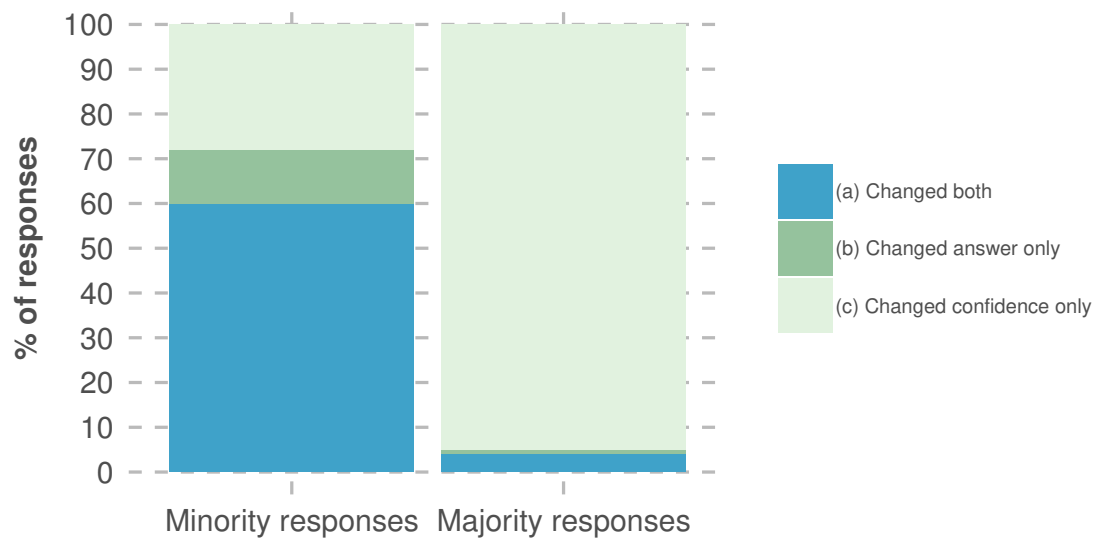

Figure 3: Distribution of changed responses across three post-feedback response types.

Upon receiving group feedback, participants could (a) change both answer option and confidence level, (b) change only their answer option, (c) change only the confidence level, 
or make no change to the initial answer or confidence. We observed that $92 \%$ (46 out of 50) of participants changed their initial response (answer option and/or confidence) at least once during the quiz, resulting in a total of 277 changes with a an average of 5.54 changes $(\mathrm{SD}=4.14)$ per person. Out of these 277 changes, 183 were made by participants placed in minorities and the remaining 94 from participants placed in majorities. The distribution of changed responses across three types on post-feedback responses (see a,b and c above) is given in Fig. 3. As expected, the distribution shows that acts of conformity (changing one's answer) occurred predominantly when participants were placed in a minority.

\subsection{Model Construction}

We consider 15 predictors as based on the presented feedback charts, participant demographics, and results from the Big-5 personality test (OCEAN). We describe these predictors in detail below:

- Majority size: Size of the majority in percentage. Range 40\%-90\%.

- Group size: Size of the group to which the participant was assigned in a given question item (could be either the majority group or a minority group).

- Group difference: Difference between the majority group size and the size of the participant's group.

- Number of minorities: Number of groups in addition to the majority group. Either 1 or 2.

- Minority one: Size of minority one. Range 5\%-40\%.

- Minority two: Size of minority two (considered only in situations where there were two minorities). Range $5 \%-40 \%$.

- Question type: Subjective or objective question type.

- Initial confidence: Participant's confidence in their answer prior to revealing the distribution of group answers. Range $0-100$.

- Gender: Participant's self-disclosed gender.

- Openness: Describes creativity and openness to new experiences. Range $1-100$.

- Conscientiousness: Describes diligent and goal-directed behaviour. Range $1-100$.

- Extraversion: Explains emotional expressiveness and outgoing social behaviour. Range $1-100$.

- Agreeableness: Captures cooperative and considerate behaviour. Range 1 - 100.

- Neuroticism: Captures emotional instability, anxiety and stress levels. Range 1 100.

- User id: An unique identifier assigned to a given user during the quiz.

We used the $\mathrm{R}$ package lme4 [43] to perform a generalised linear mixed-effects model (GLMM) analysis of the relationship between the aforementioned predictors and participant conformity. A GLMM allow us to identify the effect of a set of predictors on an outcome variable (conformity) while following an arbitrary (i.e. possibly non-normal) distribution. We considered a change in the initial answer option (with or without a change in initial 
confidence level) to that of the majority, as an indication of conformity behaviour. We observed that in some situations participants also changed their confidence on the selected answer without conforming to the majority's answer option. We specified participant (User id) as a random effect as to allow for individual differences in our model.

Following model selection (incremental removal of variables based on their predictive power), a total of five variables remained. The regression formula of the final model is illustrated by Equation 1. The estimate values, standard errors (SE), z-values, and p-values of the final model variables are given in Table 1 . We performed a likelihood ratio test with the null model [44] and found that our model is statistically significant $\left(\chi^{2}(4)=72.76\right.$, $\mathrm{p}<0.001)$ and explains $39.2 \%$ of the variance in accuracy $\left(\mathbb{R}=0.626, \mathbb{R}^{2}=0.392\right)$. From these variables, 'Question type' had the largest effect on participant conformity. Participants were more likely to conform when presented with objective questions as compared to subjective questions. To ensure the validity of the model, we checked for the existence of multicollinearity. Our predictors report a variance inflation factor between 1.04 and 1.20, well below the often-used threshold of 5 to detect multicollinearity [45].

$$
Y=-6.995+1.847 X_{1}+0.060 X_{2}-0.035 X_{3}+0.017 X_{4}+0.017 X_{5}
$$

Table 1: Effect of predictors on participant conformity.

\begin{tabular}{lllll}
\hline & Estimate & $\mathrm{SE}$ & $\mathrm{z}$ value & $\operatorname{Pr}(>|\mathbf{t}|)$ \\
\hline (Intercept) & -6.995 & 1.019 & -6.861 & $<0.001^{* * *}$ \\
Question type (objective) $\left(X_{1}\right)$ & 1.847 & 0.296 & 6.251 & $<0.001^{* * *}$ \\
Majority size $\left(X_{2}\right)$ & 0.060 & 0.009 & 6.441 & $<0.001^{* * *}$ \\
Initial confidence $\left(X_{3}\right)$ & -0.035 & 0.005 & -7.412 & $<0.001^{* * *}$ \\
Conscientiousness $\left(X_{4}\right)$ & 0.017 & 0.007 & 2.298 & $0.022^{*}$ \\
Neuroticism $\left(X_{5}\right)$ & 0.017 & 0.007 & 2.294 & $0.022^{*}$ \\
\hline
\end{tabular}

\subsection{Feature Description}

Following model construction, we present a more detailed look at the significant features. We only considered the responses which placed participants in a minority, as the dependent variable was determining conformity behaviour. We observed that contextual determinants such as the nature of the question and the majority size significantly impact the likelihood of an individual conforming to the majority in online settings. Moreover, personal determinants such as initial confidence on the answer, neuroticism, and conscientiousness displayed significant influence on online social conformity.

\subsubsection{Contextual Determinants}

The nature of the question (either objective or subjective) had the highest effect on whether an individual would conform to the majority or not. We observed that $83 \%$ (98 out of 118) of the conformity responses were related to objective questions while only $17 \%$ (20 


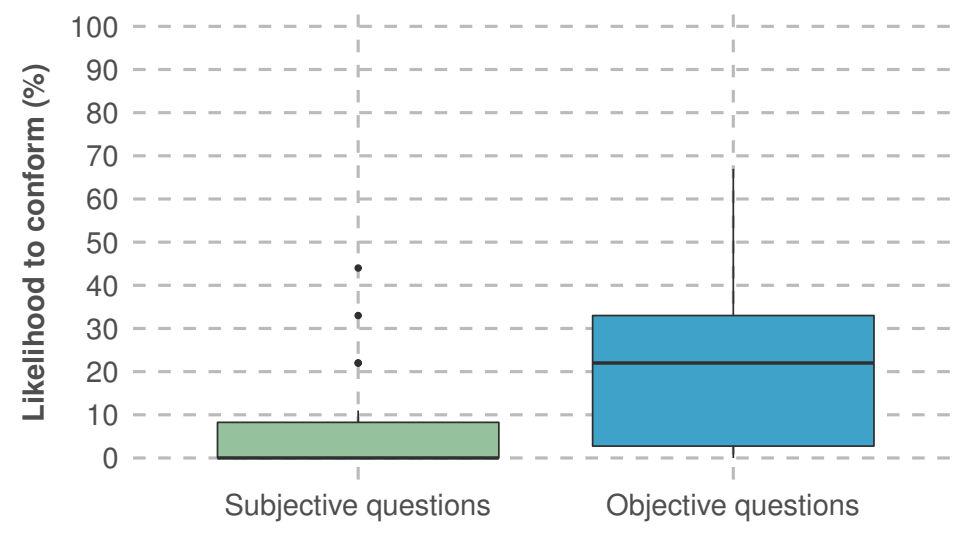

\begin{tabular}{ccc}
\hline & Subjective questions & Objective questions \\
\hline Range & $0-22$ & $0-67$ \\
IQR & $0-11$ & $0-33$ \\
Median & 0 & 22 \\
Mean & 4.4 & 21.7 \\
SD & 9.2 & 19.9 \\
\hline
\end{tabular}

Figure 4: The proportion of conformity responses per user across objective and subjective questions.

out of 118) were associated with subjective questions. Moreover, we plotted the likelihood of each participant conforming to objective and subjective questions as illustrated in Fig. 4.

We note that the likelihood of participants conforming to objective questions ranged between $0 \%-67 \%$ with a median of $22 \%$. However, the likelihood of participants conforming to subjective questions was considerably lower with a range of $0 \%-22 \%$ and a median value of $0 \%$. Additional information on the interquartile range (IQR), mean values, and the standard deviation (SD) of the two distributions are given below the box plots seen in Fig. 4. In summary, the participants were more likely to accept the majority's judgement in objective questions when compared to subjective questions.

Majority size is another contextual predictor that displayed a significant relationship with the likelihood of a person conforming to the majority. Even though we included several other contextual predictors with regard to the group distribution such as the number of minorities and their corresponding sizes and the size difference between the participant's group and the majority group, none of these predictors displayed a significant impact on our dependent variable.

Fig. 5 illustrates an upward trend in conformity as the majority group size increases from $40 \%-90 \%$, establishing that the likelihood of an individual conforming to the majority's answer increases as the majority group increased in size. This observation is in line with the existing literature $[22,5,33]$. We considered the original group size associated to the majority, before including the adjustment factor when plotting the figure. There was never a unanimous majority, as the feedback included the participant's selection as well. Moreover, as the number of group distributions that corresponded to each of the aforementioned 
majority group sizes varied, we defined likelihood of conformity as the proportion of conformity responses as a fraction of the total responses for each majority group size, for each participant.

However, it should be noted that the model also identified statistically significant main effects from several other factors such as question type, self-confidence, and personality traits of participants, which explain the outliers in Fig. 5. For example, for a given group composition half of the participants may have answered a subjective question, while the other half may have answered an objective question. Moreover, the model indicates that question type (either objective or subjective) had the largest effect on conformity behaviour.

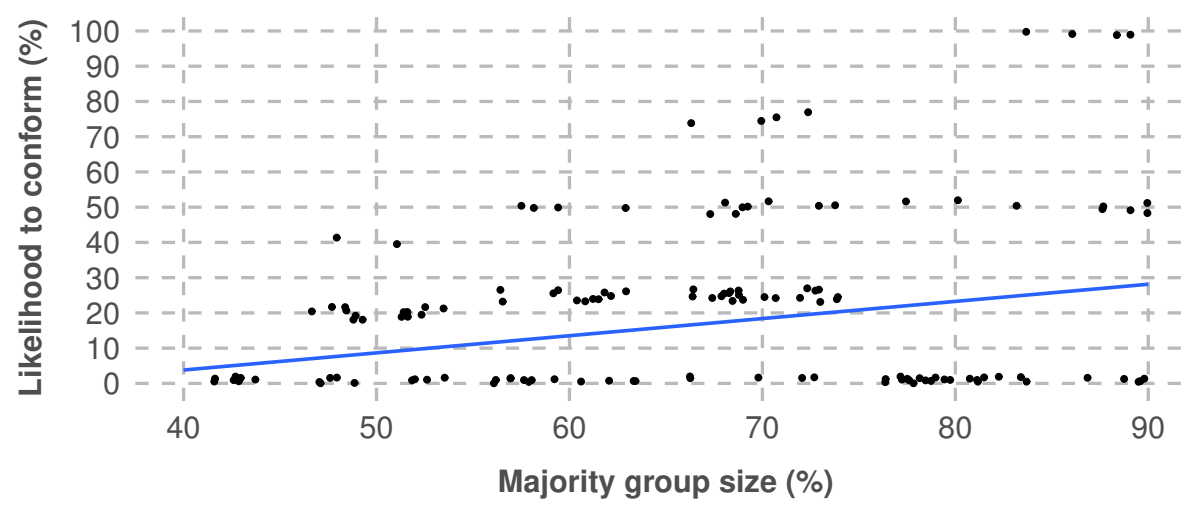

Figure 5: The likelihood of conformity in participants as opposing majority size increases.

\subsubsection{Personal Determinants}

The model established that the initial self-reported confidence level of participants has a negative correlation with the likelihood of them conforming to the majority's judgements. This notion is illustrated in Fig. 6(a) in detail. The confidence levels of participants who conformed to the majority ranged between $0-100$, with a median of 58 . where as those who did not conform to the majority demonstrated a median value of 80 with a range of confidence values from 15 - 100. In general, individuals who displayed higher confidence on their personal answers were less likely to be impacted by the majority. The interquartile range (IQR), mean values, and standard deviation (SD) of the intial confidence values for non-conforming and conforming response distributions are provided below the box plots seen in Fig. 6(a).

In addition to the aforementioned variables, the model also highlighted statistically significant relationships between personality traits such as conscientiousness $(\mathrm{C})$ and neuroticism $(\mathrm{N})$, and conformity behaviour. The other personality traits did not display statistically significant effects on conformity behaviour. The distribution of the percentile values of the scores for $\mathrm{C}$ and $\mathrm{N}$ across conforming and non-conforming behaviour of participants is as illustrated in Fig. 6(b) and (c) respectively. The corresponding statistics for the range, interquartile range (IQR), mean values, and standard deviation (SD) of the non-conforming and conforming response distributions are provided along with the box plots. For C, medians of 50 and 63 were observed from non-conforming and conforming responses respectively. 
Similarly for N, medians 50 and 64 were observed from non-conforming and conforming responses respectively. In summary, participants placed in higher percentiles for $\mathrm{C}$ and $\mathrm{N}$ were more susceptible to conformity. Moreover, we did not observe any significant gender differences in conformity behaviour of participants.
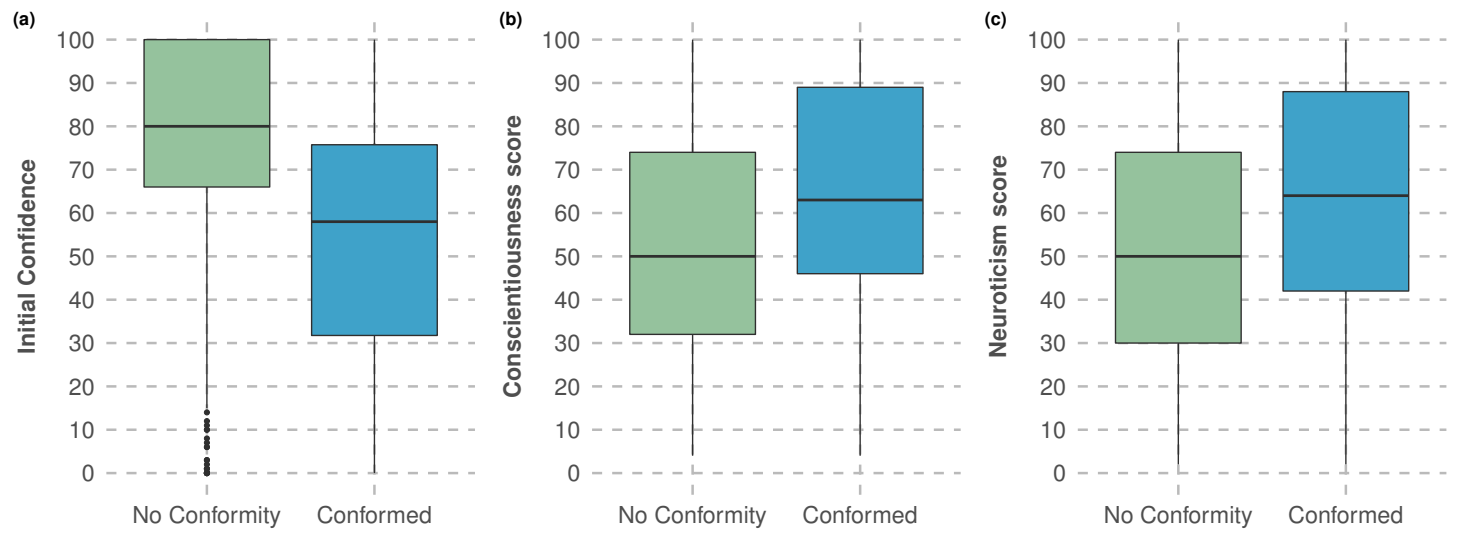

\begin{tabular}{ccccccc}
\hline & \multicolumn{2}{c}{ Initial Confidence } & \multicolumn{2}{c}{ Conscientiousness } & \multicolumn{2}{c}{ Neuroticism } \\
\hline & No Conformity & Conformed & No Conformity & Conformed & No Conformity & Conformed \\
\hline Range & $15-100$ & $0-100$ & $4-100$ & $4-100$ & $2-100$ & $2-100$ \\
IQR & $66-100$ & $31-76$ & $32-74$ & $46-90$ & $30-74$ & $42-88$ \\
Median & 80 & 58 & 50 & 63 & 50 & 64 \\
Mean & 77.5 & 54.9 & 52.8 & 60.3 & 52.0 & 59.3 \\
SD & 24.3 & 29.8 & 28.7 & 30.3 & 29.1 & 27.7 \\
\hline
\end{tabular}

Figure 6: (a) Initial confidence (b) Conscientiousness score (c) Neuroticism score of participants and their conformity behaviour.

\subsection{Qualitative Results}

To better understand the factors leading to participant conformity and the use of our tool, we performed a qualitative analysis on the transcripts of the interviews. The individual interviews lasted for 10 to 15 minutes. Our semi-structured interview approach allowed participants to highlight elements which they considered important in addition to completing an identical set of questions among participants. Our questions focused on understanding the rationale behind a participant's urge to conform to the majority's judgements, as well as the usage of group feedback and the included bot. We discuss these topics in more detail below and provide exemplar citations from our participants.

\subsubsection{Support for Contextual Determinants of Conformity}

As indicated by our quantitative results, participants were more likely to conform to the majority opinion as the group size increased. Participants highlighted that larger majorities exerted more pressure to conform than smaller majorities. "I would follow the majority if it was more than $70 \%-80 \%$. If it was $55 \%$ or $45 \%$, I may be right. And I will insist on my answer." (P37). Moreover, participants mentioned that, even if they did not change their 
answer, a significant opposing majority led them to reconsider their initial answer; "When the majority was against me, in objective questions, it made me re-think and re-calculate. But when I was sure I moved on disregarding the majority." (P11).

Participants further indicated that they more frequently changed their answers for the objective questions, again confirming our quantitative results. This was motivated by the fact that it is possible for the participant's answer to be incorrect for the objective questions. In the case of subjective questions participants felt less pressure to accept the opinions of the majority. "I went with the majority for objective questions thinking it was the right answer. I did not change in subjective questions. I can have my own opinion and did not have to agree with the majority." (P31). A number of participants considered the feedback on interpreting subjective questions useful as it forced them to consider the viewpoint of the other parties. However, the analysis also suggested that anonymity and reduced social presence among group members, reduced the effects of 'normative' influences to a significant extent (especially with regard to subjective questions). "For subjective questions I won't change my answer or confidence no matter what. If it was a physical group, it would not be the case, I would want to be included and not stand out." (P22).

\subsubsection{Support for Personal Determinants of Conformity}

Not surprisingly, participants indicated that they conformed more when they were unsure about the correct answer to the question. "I looked at the feedback and thought [that] if the majority chose it, it could be more correct. [...] When I was sure, I did not change." (P44). In such situations, the majority's judgements were perceived as an additional source of information. "For some questions when I did not know anything about the field, I chose the majority as I did not have any other source of information." (P26). These observations confirms our quantitative results and suggest the significance of 'informational' influences exerted by the group majority on conformity.

\subsubsection{Group Feedback}

Following submission of their initial answer, participants were presented with the feedback chart (i.e. the supposed answers of their peers). In general, participants reported that they were comfortable viewing the feedback. "I was very comfortable with the feedback coming for objective questions. It was like a cheat sheet with statistics. It was nice to see what how others answers." (P19). Moreover, some participants highlighted that the distribution of answers were helpful in assessing their own answers and refocusing their thoughts. "If it was a math problem, you actually need to do the work and find the answer. If a lot of other people say otherwise, it [feedback] makes you reflect on your answer, which I think is good to confirm your understanding." (P22).

\subsubsection{Chatbots}

Participants were positive about the functionalities offered by the chatbot. In particular, participants highlighted its ability to structure the quiz and keep track of progress, as well as providing a general starting point for participants to ask questions. "I liked the feeling of something automated accompanying me throughout quiz. In that quiet setting, doing this 
by myself, something is caring for me, it asks me if I need help." (P18). Even participants that did not actively use the bot were aware that the bot was there to support them when required; "I ignored it. But I knew if I needed support, I could go to that chatbot." (P25).

When asked to compare instructions provided by a chatbot to those offered in a paper format, participants were predominantly in favour of the chatbot. Participants believed that interacting with a chatbot allows them to directly get the content they need, as the chatbot could narrow down the required information. Furthermore, participants enjoyed the interaction offered by the bot: "[The chatbot] is more straightforward, convenient and you feel like you are talking to another person." (P07).

\section{Discussion}

Our results establish that online social conformity is determined by several contextual and personal determinants. We observed statistically significant relationships between majority group size, nature of the question, self-reported confidence, and certain personality traits on the likelihood of conforming behaviour.

\subsection{Factors affecting Conformity}

We observed that participants conformed more in objective questions as compared to subjective questions. This could also be attributed to the work of 'informational' influence. However, in the case of 'normative' influences, our findings contradict with previous work which observed higher conformity in subjective questions with a perceived socially 'acceptable' answer supported by the majority [19]. The impact of 'normative' influence was not prominently observed during our study. Rather, participants explained that the anonymity of the online setting encouraged them to support their judgements, especially in the case of subjective questions. On that note, it should be acknowledged that the cited study considered the impact of different levels of social presence among group members on their 'normative' behaviour, which was at a minimum in our study.

We note an upward trend in conforming behaviour as the majority increases in size. These observations are consistent with the literature [22, 34]. However, it is noteworthy that our study did not merely employ unanimous majorities to influence conformity behaviour as was the case in previous work, but instead we investigated a broad spectrum of majority sizes. Furthermore, previous work has suggested that larger majorities exert higher 'informational' influence [37]. Our qualitative analysis confirmed this hypothesis. Participants rationalised their conformity behaviour (especially in objective questions) as the 'need to be right' and emphasised that the answers provided by larger majorities were more plausible. As the majority grew in size, it was perceived unlikely to be wrong. Moreover, the presence of multiple minorities with varying group sizes had no significant impact on conformity.

During the quiz, participants reported their self-confidence on their answers. Participants who were unsure and less confident on their selections conformed more frequently to an opposing majority's judgements. Based on this observation we infer that lower selfconfidence on personal judgements amplifies the effects of 'informational' influences, and individuals who are less confident on personal judgements can easily be swayed towards 
that of the majority. This was also validated by our qualitative analysis where participants explained that when unsure of the chosen answer, the majority's judgement appeared to be a more likely source of 'right' information. Similar observations were made by Rosander and Eriksson [20] where online conformity was more prominent as the self-reported difficulty of tasks increased.

Existing literature concerning personal traits and conformity suggests that higher anxiety could be a significant determinant of social conformity [31, 46]. Our results confirm this premise, as we observed neuroticism scores of participants (which describes anxiety and emotional stability) to have a strong positive correlation with their conformity behaviour. This suggests that individuals who are less emotionally stable are more susceptible to conformity influences. Moreover, a similar relationship was observed between conscientiousness scores (which describes goal-orientation and diligence) and conformity. Such goal-oriented behaviour may encourage an individual to obtain the 'correct' answer at any cost. Individuals with high conscientiousness may doubt their answers when facing a contradicting majority and accept the majority's judgement to be more accurate than their own perception of the same situation. This behaviour is likely to be encouraged by 'informational' influences at play.

Furthermore, literature on face-to-face conformity highlights gender differences in conformity behaviour, driven by stereotypical masculine and feminine social roles imposed by society [26]. However, our results contradict this notion as we do not observe statistically significant differences in conformity amongst men and women. We emphasise that the online setup used for this study lacks the social presence introduced in typical face-to-face group settings, which may have reduced the influence of the aforementioned gender stereotypical social roles on the observed conformity behaviour of our participants.

\subsection{Online Social Conformity}

In general, the results of this study strongly indicate that online social conformity is a function of multiple contextual and personal determinants. While the inherent dissimilarities between face-to-face and online groups (such as anonymity and reduced social presence in the latter), may have reduced the effects of 'normative' influences, 'informational' influences are still predominantly apparent in online settings. This study showed that the 'need to be right' displayed strong associations with all the aforementioned predictors. Thus, our results suggest that online settings with static and unidirectional communication is sufficient to elicit conformity behaviour mainly influenced by 'informational influences' (rather than 'normative influences'), and that by regulating the determinants it may be possible to reduce the impact of 'informational' influences on social conformity.

Moreover, in our qualitative analysis we observed that participants generally preferred receiving feedback from peers and perceived it as a tool of learning and reasoning. This observation follows [47] and [48], that explain the advantages of peer feedback in group settings. Thus, completely eliminating the use of feedback may not be the best approach to avoid conformity. Further work is required to identify online environments where conformity possesses a higher risk and employ methods that can mitigate its adverse impacts. Such 
methods could vary from simple design adjustments in online communities [15] to more complex techniques that reduce the anticipated impact of the contextual (e.g., anonymity, use of visual cues, diversity, group size) and personal (e.g., self-confidence, personality differences) determinants of social conformity.

Finally, the use of a chatbot for training and support during the quiz was seen as beneficial, as a significant number of participants preferred instructions coming from an interactive bot over static on-screen instructions, which is in line with related literature [49]. It also enabled us to simulate a more realistic online environment where participants understood the instructions and familiarised themselves with the setting, minimising any influence by the researcher (i.e. Hawthorne Effect [50]), a crucial aspect when investigating social conformity. Furthermore, our participants perceived the chatbot as a readily available source of support and feedback in an isolated setting. Similar observations were made in $[51,52]$. Some participants also mentioned that even the simple interpretations given by the bot regarding the feedback charts assisted them in reasoning and decision making as previously established in [53].

\subsection{Limitations}

There were several limitations in our study. Even though our participants came from diverse backgrounds, they represented a relatively young population with adequate digital experience. This may have indirectly discouraged 'normative' social influences. Furthermore, we did not investigate aspects of online social interactions beyond anonymity in the current study. We note that future work could look into the effects of different levels of social presence and the use of social context cues (e.g., names, avatars) in online settings and their effect on conformity behaviour. Moreover, to exclude confounding variables such as participant assertiveness, we deployed our study in a controlled environment (one participants at a time). We aim to explore the effect of simultaneous interactions in an online setting in future work. Future research could also explore the effect of the identified contextual and personal determinants on the quality of output generated by online groups.

\section{Conclusion}

Social conformity is a widely experienced form of social influence, both in face-to-face and online groups, where minorities change their behaviour and opinions to match contrasting opinions of the group majority. While determinants of conformity has been studied in faceto-face groups, it is yet to be thoroughly explored in online group settings. Thus, this work aimed to study both contextual and personal determinants of social conformity and their implications in online environments.

Our results establish that larger majority group sizes have a bigger effect on conformity behaviour. Participants conformed more frequently for objective questions demonstrating high levels of 'informational' influences. Moreover, participants who reported low self-confidence, demonstrated high conscientiousness, or had high levels of neuroticism commonly conformed to the majority. We observed no significant effects rising from the number 
of minorities or minority group sizes. Moreover, no strong gender differences were observed with regard to conformity behaviour.

Our observations concerning majority group sizes, self-reported confidence, and personality traits are in line with existing literature related to 'informational' influences of conformity. However, with regard to the nature of questions our work presents contrasting findings to those in the literature. Our qualitative analysis suggests that this difference in behaviour may be due to lower levels of 'normative' influences in an online setting as opposed to physical groups.

Our work set forth several avenues for further work. We intentionally utilised anonymous peers to suit the research objectives explored in this study. However, further work could investigate online social conformity when peers are identifiable through realistic cues (such as first names, usernames and avatars). Such factors could trigger stereotypical behaviour with regard to gender and age which could enhance or diminish conformity influences. Moreover, the cues themselves may differ from one another based on the amount of influence they exert. This would be a potential step forward in understanding factors of social conformity in realistic online settings.

\section{References}

[1] S. E. Asch, Groups, leadership and men, Carnegie Press, Oxford, England, 1951, chapter: Effects of group pressure upon the modification and distortion of judgements, pp. 177-190.

[2] M. Deutsch, H. B. Gerard, A study of normative and informational social influences upon individual judgment., The journal of abnormal and social psychology 51 (3) (1955) 629-636.

[3] M. F. Kaplan, C. E. Miller, Group decision making and normative versus informational influence: Effects of type of issue and assigned decision rule., Journal of Personality and social psychology 53 (2) (1987) 306-313.

[4] S. E. Asch, Studies of independence and conformity: A minority of one against a unanimous majority., Psychological monographs: General and applied 70 (9) (1956) 1-70.

[5] H. B. Gerard, R. A. Wilhelmy, E. S. Conolley, Conformity and group size., Journal of Personality and Social Psychology 8 (1) (1968) 79-82.

[6] R. R. Blake, H. Helson, J. S. Mouton, The generality of conformity behavior as a function of factual anchorage. difficulty of task, and amount of social pressure, Journal of Personality 25 (3) (1957) $294-$ 305.

[7] J. F. Coleman, R. R. Blake, J. S. Mouton, Task difficulty and conformity pressures., The Journal of Abnormal and Social Psychology 57 (1) (1958) 120-122.

[8] A. H. Eagly, C. Chrvala, Sex differences in conformity: Status and gender role interpretations, Psychology of Women Quarterly 10 (3) (1986) 203-220.

[9] L. A. Rosenberg, Conformity as a function of confidence in self and confidence in partner, Human Relations 16 (2) (1963) 131-139.

[10] R. S. Crutchfield, Conformity and character., American Psychologist 10 (5) (1955) 191.

[11] B. Reynolds, J. Venkatanathan, J. Goncalves, V. Kostakos, Sharing ephemeral information in online social networks: Privacy perceptions and behaviours, in: Proceedings of the IFIP Conference on HumanComputer Interaction - INTERACT 2011, 2011, pp. 204-215.

[12] J. Goncalves, V. Kostakos, J. Venkatanathan, Narrowcasting in social media: Effects and perceptions, in: Proceedings of the 2013 IEEE/ACM International Conference on Advances in Social Networks Analysis and Mining, ASONAM '13, 2013, pp. 502-509.

[13] K. Y. McKenna, A. S. Green, Virtual group dynamics., Group dynamics: theory, research, and practice 6 (1) (2002) 116-127. 
[14] T. Beran, M. Drefs, A. Kaba, N. Al Baz, N. Al Harbi, Conformity of responses among graduate students in an online environment, The Internet and Higher Education 25 (2015) 63-69.

[15] A. Sukumaran, S. Vezich, M. McHugh, C. Nass, Normative influences on thoughtful online participation, in: Proceedings of the SIGCHI Conference on Human Factors in Computing Systems, CHI '11, ACM, New York, NY, USA, 2011, pp. 3401-3410.

[16] E. Sharma, M. De Choudhury, Mental health support and its relationship to linguistic accommodation in online communities, in: Proceedings of the 2018 CHI Conference on Human Factors in Computing Systems, CHI '18, ACM, New York, NY, USA, 2018, pp. 641:1-641:13.

[17] M. Cinnirella, B. Green, Does 'cyber-conformity'vary cross-culturally? exploring the effect of culture and communication medium on social conformity, Computers in Human Behavior 23 (4) (2007) 20112025.

[18] P. B. Lowry, T. L. Roberts, N. C. Romano Jr, P. D. Cheney, R. T. Hightower, The impact of group size and social presence on small-group communication: Does computer-mediated communication make a difference?, Small Group Research 37 (6) (2006) 631-661.

[19] L. Laporte, C. van Nimwegen, A. J. Uyttendaele, Do people say what they think: Social conformity behavior in varying degrees of online social presence, in: Proceedings of the 6th Nordic Conference on Human-Computer Interaction: Extending Boundaries, NordiCHI '10, ACM, New York, NY, USA, 2010, pp. 305-314.

[20] M. Rosander, O. Eriksson, Conformity on the internet-the role of task difficulty and gender differences, Computers in human behavior 28 (5) (2012) 1587-1595.

[21] O. P. John, S. Srivastava, The big five trait taxonomy: History, measurement, and theoretical perspectives, Handbook of personality: Theory and research 2 (1999) (1999) 102-138.

[22] S. E. Asch, Opinions and social pressure, Scientific American 193 (5) (1955) 31-35.

[23] J. M. Levine, Solomon asch's legacy for group research, Personality and Social Psychology Review 3 (4) (1999) 358-364.

[24] R. B. Cialdini, N. J. Goldstein, Social influence: Compliance and conformity, Annu. Rev. Psychol. 55 (2004) 591-621.

[25] R. Bond, Group size and conformity, Group processes \& intergroup relations 8 (4) (2005) 331-354.

[26] A. H. Eagly, W. Wood, Gender and influenceability: Stereotype versus behavior, in: Women, gender, and social psychology, Erlbaum, Hillsdale, NJ, USA, 1985, pp. 225-256.

[27] A. H. Eagly, Gender and social influence: A social psychological analysis., American Psychologist 38 (9) (1983) 971-981.

[28] F. Samelson, Conforming behavior under two conditions of conflict in the cognitive field., The Journal of Abnormal and Social Psychology 55 (2) (1957) 181-187.

[29] B. Mausner, The effect of prior reinforcement on the interaction of observer pairs., The Journal of Abnormal and Social Psychology 49 (1) (1954) 65-68.

[30] B. Mausner, B. L. Bloch, A study of the additivity of variables affecting social interaction., The Journal of Abnormal and Social Psychology 54 (2) (1957) 250-256.

[31] C. Meunier, B. G. Rule, Anxiety, confidence, and conformity, Journal of Personality 35 (3) (1967) 498-504.

[32] M. Smilowitz, D. C. Compton, L. Flint, The effects of computer mediated communication on an individual's judgment: A study based on the methods of asch's social influence experiment, Computers in Human Behavior 4 (4) (1988) 311-321.

[33] D. J. Stang, Group size effects on conformity, Journal of Social Psychology 98 (2) (1976) 175-181.

[34] B. Latané, S. Wolf, The social impact of majorities and minorities., Psychological Review 88 (5) (1981) 438-453.

[35] L. W. Ferguson, An analysis of the generality of suggestibility to group opinion, Journal of Personality 12 (3) (1944) 237-243.

[36] R. R. Blake, H. Helson, J. S. Mouton, The generality of conformity behavior as a function of factual anchorage. difficulty of task, and amount of social pressure, Journal of Personality 25 (3) (1957) 294305. 
[37] C. A. Insko, R. H. Smith, M. D. Alicke, J. Wade, S. Taylor, Conformity and group size: The concern with being right and the concern with being liked, Personality and Social Psychology Bulletin 11 (1) (1985) 41-50.

[38] N. S. Endler, Conformity analyzed and related to personality, The Journal of Social Psychology 53 (2) (1961) 271-283.

[39] Y. Liu, J. Venkatanathan, J. Goncalves, E. Karapanos, V. Kostakos, Modeling what friendship patterns on facebook reveal about personality and social capital, ACM Trans. Comput.-Hum. Interact. 21 (3) (2014) $17: 1-17: 20$.

[40] J. Venkatanathan, E. Karapanos, V. Kostakos, J. Goncalves, Network, personality and social capital, in: Proceedings of the 4th Annual ACM Web Science Conference, WebSci '12, ACM, 2012, pp. 326-329.

[41] L. Adrianson, Gender and computer-mediated communication: Group processes in problem solving, Computers in Human Behavior 17 (1) (2001) 71-94.

[42] D. J. Stang, Ineffective deception in conformity research: Some causes and consequences, European Journal of Social Psychology 6 (3) (1976) 353-367.

[43] D. Bates, M. Mächler, B. Bolker, S. Walker, Fitting linear mixed-effects models using lme4, Journal of Statistical Software 67 (1) (2015) 1-48.

[44] B. M. Bolker, M. E. Brooks, C. J. Clark, S. W. Geange, J. R. Poulsen, M. H. H. Stevens, J.-S. S. White, Generalized linear mixed models: a practical guide for ecology and evolution, Trends in Ecology \& Evolution 24 (3) (2009) 127-135.

[45] J. F. Hair, W. C. Black, B. J. Babin, R. E. Anderson, R. Tatham, Multivariate Data Analysis, Pearson, New Jersey, NJ, USA, 2010.

[46] B. G. Rule, M. L. Sandilands, Test anxiety, confidence, commitment, and conformity, Journal of Personality 37 (3) (1969) 460-467.

[47] M. London, V. I. Sessa, Group feedback for continuous learning, Human Resource Development Review 5 (3) (2006) 303-329.

[48] E. Van Popta, M. Kral, G. Camp, R. L. Martens, P. R.-J. Simons, Exploring the value of peer feedback in online learning for the provider, Educational Research Review 20 (2017) 24-34.

[49] H. van der Meij, Motivating agents in software tutorials, Computers in Human Behavior 29 (3) (2013) $845-857$.

[50] J. G. Adair, The hawthorne effect: a reconsideration of the methodological artifact., Journal of applied psychology 69 (2) (1984) 334-345.

[51] J. Pereira, Leveraging chatbots to improve self-guided learning through conversational quizzes, in: Proceedings of the Fourth International Conference on Technological Ecosystems for Enhancing Multiculturality, ACM, 2016, pp. 911-918.

[52] T. W. Bickmore, L. M. Pfeifer, M. K. Paasche-Orlow, Using computer agents to explain medical documents to patients with low health literacy, Patient education and counseling 75 (3) (2009) 315320.

[53] N. T. Le, L. Wartschinski, A cognitive assistant for improving human reasoning skills, International Journal of Human-Computer Studies 117 (2018) 45-54. 


\section{Chapter 5}

\section{Age as a Determinant of Conformity}

\subsection{Introduction}

The findings presented in Chapter 4 show that online social conformity should be investigated in terms of both its contextual and personal determinants. However, prior work has shown little interest in investigating certain personal determinants of social conformity, such as age (see Table 2.2). Moreover, despite being identified as an important conformity determinant in physical groups [91, 132], we found only one CMCbased conformity study that at least accounted for potential effects of user age on their conformity behaviour (see Table 2.2). Furthermore, user age has been referred to as an indicator of user "status" in face-to-face conformity studies [47] that can impact how users perceive both self and peer "superiority" in a task. Thus, we argue that effects of age on conformity should be investigated accounting for potential age-stereotypical perceptions of self and peer competency. This notion is further corroborated by recent literature that indicate that users stereotypically perceiving competency and trustworthiness of online peers based on peer age $[65,131]$.

Therefore, in this chapter we systematically investigate effects age and related stereotypes on user conformity behaviour in an online Instant Messaging platform, across three aspects - user's age group (Generation X and Generation Z), age group composition of peers challenging user responses (Generation X or Generation Z or mixed), and the stereotypically perceived age of task (MCQs that are stereotypically perceived to be better known to Generation X, Generation Z, and neutral). We further note that user/peer age was only implied using their birth years which were embedded on to their usernames (e.g., e1p1_1998, e1p2_1965). Our results indicate that both Generation $\mathrm{X}$ and Generation $\mathrm{Z}$ users conform to contradictory responses of peers belonging to the opposite age generation (i.e. Generation $\mathrm{Z}$ or X respectively), only when peers' age group aligns with the stereotypically perceived age demographic of a question. Additionally, the inverse relationship between confidence on personal answer and user conformity behaviour reported in Article I also emerged in this study.

We elaborate on our findings and their implications for designing future online group settings that account for age-stereotypical conformity, in the attached publication, Article II. In brief, our results show that people infer peer age using minimal user cues (e.g. birth years) to stereotypically perceive both self and peer competency - especially in stereotypically age-biased tasks. Such perceptions can consequently determine their receptiveness to opposing views of peers in online groups. Hence, designers of online group settings should be mindful about the user cues they expose through platform design - especially in situations where age-stereotypical conformity can lead to adverse effects (e.g. unwarranted biases against certain age generations [65, 131]). 


\subsection{Article II}

Copyright is held by the International Federation for Information Processing 2021. Published by Springer Nature Switzerland AG 2021. This is the authors' version of the work. It is posted here for your personal use. Not for redistribution. The definitive Version of Record was published in:

Wijenayake S., Hu J., Kostakos V., Goncalves J. (2021). Quantifying the Effects of AgeRelated Stereotypes on Online Social Conformity. In: Ardito C. et al. (eds) HumanComputer Interaction - INTERACT 2021. INTERACT 2021. Lecture Notes in Computer Science, vol 12935. Springer, Cham. https://doi.org/10.1007/978-3-030-85610-6_26.

Ethics ID: 2056161, The University of Melbourne Human Ethics Advisory Group. 


\title{
Quantifying the Effects of Age-related Stereotypes on Online Social Conformity
}

\author{
Senuri Wijenayake, Jolan Hu, Vassilis Kostakos, and Jorge Goncalves \\ The University of Melbourne, Melbourne, Australia \\ \{swijenayake, jolan\}@student.unimelb.edu.au \\ \{vassilis.kostakos, jorge.goncalves\}@unimelb.edu.au
}

\begin{abstract}
Social conformity is the act of individuals adjusting personal judgements to conform to expectations of opposing majorities in group settings. While conformity has been studied in online groups with emphasis on its contextual determinants (e.g., group size, social presence, task objectivity), the effect of age - of both the individual and the members of the opposing majority group - is yet to be thoroughly investigated. This study investigates differences in conformity behaviour in young adults (Generation Z) and middle-aged adults (Generation X) attempting an online group quiz containing stereotypically age-biased questions, when their personal responses are challenged by older and younger peers. Our results indicate the influence of age-related stereotypes on participants' conformity behaviour with both young and middle-aged adults stereotypically perceiving the competency of their peers based on peer age. Specifically, participants were more inclined to conform to older majorities and younger majorities in quiz questions each age group was stereotypically perceived to be more knowledgeable about (1980's history and social media \& latest technology respectively). We discuss how our findings highlight the need to re-evaluate popular online user representations, to mitigate undesirable effects of age-related stereotypical perceptions leading to conformity.
\end{abstract}

Keywords: social conformity $\cdot$ peer age $\cdot$ age stereotypes $\cdot$ user cues $\cdot$ bots

\section{Introduction}

Social conformity is a powerful social influence that encourages individuals to change their personal judgements when challenged by an opposing group majority [3,4]. Researchers explain that individuals conform either because they perceive information supported by the majority to be 'correct' (informational conformity), or as they attempt to 'fit in' with a group to ensure their membership (normative conformity) [23,77,78,79]. While preliminary studies of social conformity were initially based on face-to-face groups $[3,4,9,23,37]$, as a significant proportion of human societal interactions are now taking place through diverse online group settings (e.g., social networks, online chatrooms, discussion forums) $[5,15,28,29,51,60,73]$, understanding repercussions of social conformity on online group interactions is of growing interest to the HCI research community.

Recent literature has studied conformity behaviour across a wide variety of online groups such as social media $[19,52,53,80,81]$, learning platforms $[8,77,78,79]$, news websites [69], and support groups [65]. However, the majority of these studies have focused on 
quantifying online social conformity in terms of its contextual determinants such as majority group size $[61,78,80]$, social presence $[45,79]$ and task objectivity $[45,61,78,79]$. Conversely, less emphasis has been placed on determining how more personal factors - that have been shown to elicit stereotypical perceptions in online communities (e.g., age [2,13], gender $[16,49,77]$, culture [17], race [20]) - influence online conformity behaviour.

In particular, age of an individual has been recognised as a vital determinant of one's susceptibility to conformity influences in offline groups [22,41,42,43,74]. Prior work indicate a non-linear relationship between an individual's age and their conformity behaviour, where susceptibility to conformity is seen to increase with age till adolescence, after which it gradually declines [22,74]. Furthermore, studies investigating age differences among adults observe higher conformity behaviour in older adults, than in their younger counterparts $[41,42,43]$. These studies rationalise that conformity behaviour runs parallel with socialisation processes that individuals follow to integrate themselves in the community (e.g. young children rely on peers to determine their behaviour in groups - leading to higher conformity, whereas by early adulthood they tend to be more confident of their own actions - reducing susceptibility to conformity influences [22]). However, it is unclear if these observations would hold in online groups where social processes may not be obvious or equally strong due to inherently lower social presence and higher anonymity [54]. Moreover, there is evidence in literature that individuals tend to stereotypically perceive competency and trustworthiness of online peers based on peer age $[30,57]$. Other conformity studies also indicate that similar stereotypical perceptions of peer competency (triggered based on peer gender) can exacerbate online conformity behaviour and lead to incorrect judgements [77,49]. However, such effects are yet to be investigated with regard to age-related stereotypes in online groups.

Therefore, this study takes an initial step towards quantifying effects of age and related stereotypes on online social conformity. We investigate potential differences in conformity behaviour among two distinct age generations - young adults (Generation Z) and middle-aged adults (Generation X) - when completing an online quiz delivered through an Instant Messaging (IM) platform, in small groups. We intend to understand whether and how people infer age of their online peers, and use this information to determine their conforming or non-conforming behaviour against younger/older peers who support a contradicting judgement. This understanding is critical to design future online group platforms that account for possible detrimental effects of age-related stereotypes (e.g. unfair treatment of older adults who are perceived as less reliable and trustworthy than their younger counterparts $[57,30,34])$, to ensure positive societal interactions.

\section{Related Work}

Despite the enhanced anonymity and reduced social presence offered by online platforms [54], individuals are susceptible to both informational and normative conformity influences in online groups settings $[8,19,48,52,53,65,78,81,82]$. For example, students completing group quizzes in online learning platforms have been seen to conform to the majority's responses, in an attempt to obtain more 'correct' answers [8,78,79]. Recent work has also shown that Facebook users tend to accept the majority's negative or positive perception of a news article's trustworthiness (inferred through user comments posted underneath the article) as a benchmark to differentiate between fake and real news articles shared on the platform $[19,80]$. The above studies emphasise the 
significance of informational influences (or the need to be 'right') [23] in prompting conformity behaviour in online group settings. On the other hand, Zhu et al. [82] observe that individuals tend to align their online choices with those of the opposing majority's - even when required to make choices based on personal preference - indicating the presence of normative conformity influences (or the need to be 'liked') [23]. Similarly, normative conformity has been observed in online support groups, where users tend to conform to community-accepted conventions of behaviour and linguistic norms, with the intention of receiving better support and feedback from other community members [65].

Moreover, prior work investigating implications of online social conformity suggests the potential for both negative and positive effects $[8,35,69,77]$. For instance, a recent study examining the effect of social information on the accuracy of a visual judgement task highlights that conforming to biased and incorrect responses from peers led to more errors among Mechanical Turk users [35]. Similar observations were noted in students who wrongly assumed the majority to be 'correct' when attempting online quizzes (informational conformity), and obtained more incorrect answers than those who attempted the quiz independently $[8,77,78]$. Conversely, normative conformity is considered useful to encourage users of online news websites to follow accepted norms of the community and contribute high quality and 'thoughtful' content [69].

Therefore, it is critical to understand what contextual and personal factors affect susceptibility of individuals to online conformity influences, in order to minimise its detrimental effects on online societal interactions (e.g. undue pressure to conform to majority's incorrect judgements). However, the majority of prior studies focus on contextual determinants of online conformity i.e., majority group size, task objectivity (subjective or objective nature of a task) and social presence (sense of being connected with others in the group [66]) $[8,45,61,78,79]$. In brief, these studies indicate that participants are more likely to conform when challenged by larger majorities, as they attempt objective tasks (with a specific correct answer), and in online settings with higher perceived social presence.

Moreover, several studies investigating personal determinants of conformity indicate effects of users' self-confidence and gender $[49,61,77,78,79,80]$. These studies unanimously note that participants with higher confidence on personal decisions are significantly less likely to conform when challenged by opposing majorities $[77,78,79,80]$. Furthermore, while no significant differences in conformity behaviour is observed among men and women in online groups $[61,78,79]$, prior work note that both men and women are more inclined to conform when challenged by male-dominant and female-dominant majorities, in stereotypically masculine and feminine tasks respectively [49,77]. Findings from these studies imply that when competency of online peers is not explicitly known, users tend to stereotypically perceive peer competency based on available user cues (i.e. in this case, user gender derived from their first name or stereotypically gendered avatar) - especially in the presence of stereotypical tasks. These observations emphasise the need to investigate implications of other user cues (such as age) that can trigger similar stereotypical conformity behaviour in online groups.

However, online conformity literature is yet to systematically investigate effects of age on social conformity - despite age being identified as a critical conformity determinant in offline conformity literature $[41,42,43,74]$. Therefore, this study intends to take an initial step towards identifying the effects of age on online conformity behaviour. Next, we summarise the offline conformity literature investigating effects of age on social conformity, which informed the design of our study. 


\subsection{Conformity as a Function of Age}

The majority of offline conformity studies investigating effects of age on conformity behaviour have focused on identifying differences in conformity between young adults (18 22 years) and older adults (>55 years) $[41,42,43]$. These studies unanimously indicate that on perceptual tasks, older adults conform significantly more often than young adults. For instance, in a study that compared conformity behaviour in young and older adults attempting a series of visual judgement tasks, author observed that older participants were more susceptible to conformity influences than their younger counterparts [41]. Similar observations were noted by Klein and Birren [42], where older participants conformed more often than younger participants in an auditory signal detection task. On the other hand, prior studies investigating determinants of online conformity do not indicate significant effects from participant age $[8,45,46,47,49,61,78,79]$. However, as most of these studies primarily recruited young adults, it is likely that the age distribution of the recruited participants was not sufficient to reveal significant effects from participants age on their conformity behaviour. Therefore, in this study we intend to recruit participants in two distinct age generations - Generation Z (young adults between 18-23 years) and Generation X (middle-aged adults between 40-55 years) [24] - to investigate potential age differences in participants' susceptibility to online conformity influences.

Furthermore, literature also indicates that an individual's decision to conform or not, depends on their perceived self-competency in an experimental task, in comparison to the perceived competency of the opposing majority (or peers) [21]. In other words, as a result of informational influences (or the need to be 'right') individuals tend to conform to majorities they perceive as more competent or knowledgeable than they are, in a given situation. On that note, we highlight that competency of peers has been often stereotypically inferred through their age in both offline and online groups, subsequently affecting how users interact with their peers $[14,25,43]$. For instance in offline contexts, school children have been observed to assume peer competency based on peer age, and more often imitate peers they perceive as more competent than themselves [14]. Furthermore, such age-biased perceptions of peer competency have also been noted to trigger stereotypical conformity in offline groups $[41,42,43]$. For instance, Klein and Birren [43] observed that both young and older adults stereotypically assumed older peers to be less competent and reliable in visual judgement tasks (due to the perceptual nature of the activity), consequently encouraging older participants to conform more to their younger counterparts, whereas younger participants were seen less inclined to conform to their older peers. Authors also emphasised that when stereotypical perceptions regarding perceived self-competency were controlled so that young and older adults had similar perceptions of their task competency, previously observed differences in conformity diminished.

Similarly, despite the absence of face-to-face interactions, peer age has often been used to gauge peer competency and trustworthiness in online groups [30,50,57]. For instance, in a recent study investigating the impact of borrowers' personal features (e.g., age, gender, physical attractiveness) on online peer-to-peer lending decisions, authors indicate that users actively inferred age of peers through their photographs, and considered assumed age a reliable indicator of peer competency to repay the loan [30]. Similarly, Pak et al. [57] observed that users of an online health management application assumed peers represented using younger anthropomorphic (human-like) avatars as more reliable and trustworthy than peers represented using older avatars. The authors 
further explained that the above differences in preference could be due to negative stereotypes associated with older adults [59].

However, effects of peer age and related stereotypes on conformity behaviour is yet to be systematically investigated in online groups. Hence, in addition to age differences in conformity behaviour, the present study will also explore how stereotypical perceptions related to peer age and competency may impact conformity behaviour among young (Generation Z) and middle-aged adults (Generation X), as they attempt tasks that are stereotypically age-biased and perceived to be more familiar to either young or middle-aged adults.

\section{Method}

We aim to investigate the impact of three aspects of age on social conformity in an online group setting using a 2 (participants' age: young adults vs. middle-aged adults) $\mathrm{x}$ 2 (majority's age group composition: all young/middle-aged, mixed) x 3 (stereotypically perceived question type: young, middle-aged, neutral) mixed design, where participant's age and majority's age group composition are manipulated between subjects (resulting in four experimental conditions). The study was deployed as a group quiz containing multiple-choice questions (MCQ) on Slack (www.slack.com) - an online instant messaging platform - which allowed us to expose participants to stereotypically age-biased questions, in the presence of diverse age group compositions in a realistic online group environment. The decision to use a MCQ quiz for the study was inspired by recent literature which uses quizzes to investigate conformity in online group settings $[8,45,61,77,78,79]$.

\subsection{The Quiz}

The quiz contained 30 objective MCQs which were equally distributed among topics that young adults (or Generation Z) are perceived to be better at (i.e. social media \& latest technology), middle-aged adults (or Generation X) are perceived to be better at (i.e. 1980's history), and topics that are neutral or timeless (i.e. general knowledge). On that note, we emphasise that this study intentionally focused on Generations X and $\mathrm{Z}$, with an entire age generation (Generation $\mathrm{Y}$ ) separating the two age groups, to avoid potential overlaps in age-related stereotypical perceptions. Moreover, prior work has indicated that there are clearly established age-related stereotypes attached to Generations X and Z, that the aforementioned question topics have been seen to successfully trigger $[13,24,36,40,70]$. Furthermore, we chose general knowledge topics to represent neutral questions, as recent work has shown no age differences in conformity for general knowledge questions [45,61,77,78,79].

After determining topics for each question type, we created a question repository by extracting objective MCQs related to the selected topics. The neutral questions were extracted from recent online conformity literature (i.e., $[77,78,79]$ ), whereas questions that are stereotypically perceived as age-biased were extracted from popular online questions repositories i.e., Britannica, Sporcle, and Washington Times quizzes. In order to determine a final list of quiz questions that could trigger age-related stereotypical perceptions, we then followed a filtering mechanism that has been frequently used in recent studies investigating effects of stereotypes on conformity behaviour [46,47,49,77]. Two of the paper's authors (one from Generation Z and another from Generation X) independently 
rated the stereotypically age-biased questions on their familiarity to young (Generation $\mathrm{Z}$ ) and middle-aged (Generation X) adults, each on a 10-point Likert scale (1 - Gen Z/X is not at all likely to be familiar to $10-$ Gen $\mathrm{Z} / \mathrm{X}$ is extremely likely to be familiar). The familiarity score for Generation X was then reverse-coded and added to the Generation $\mathrm{Z}$ score, to arrive at a final score for each stereotypical question. Similarly, authors rated the neutral questions for their likelihood to trigger an age-related stereotypical perception (1 - Not at all likely to trigger age-related stereotypical perceptions to 10 - Extremely likely to trigger age-related stereotypical perceptions). We computed the weighted kappa $(\mathrm{kw})$ to assess the inter-coder reliability of the two raters, to note $\mathrm{kw}=0.81(95 \%$ CI, 0.72 to $0.90, p<0.001)$ - which indicates excellent agreement beyond chance $[26]$ - further validating the categorisation of the quiz questions. We then selected the top 10 questions from each topic (i.e., topics familiar to Generation $\mathrm{Z}$ or young adults, topics familiar to Generation X or middle-aged adults, and topics related to general knowledge) to be included in the quiz. By exposing individuals in groups to different questions types (stereotypically age-biased and otherwise), we intend to determine whether they would consider age as a factor of peer competency when deciding whether to conform or not, especially in topics that are stereotypically perceived to favour a certain age group. We list several questions used in the quiz representative of each question type in Table 1.

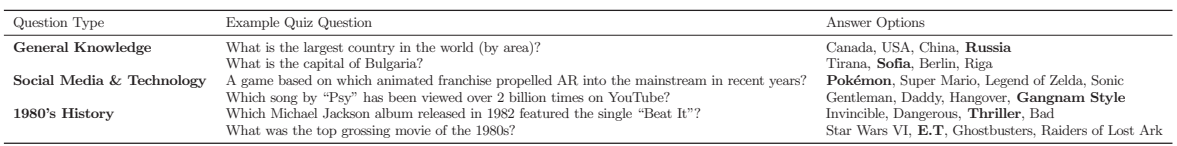

Table 1. Example questions used in the quiz. Correct answer for each question is in bold.

The quiz was conducted in a Slack channel (an online chatroom). We used a pre-programmed Slack bot named "SupportBot" to conduct the quiz without any involvement from the researchers in order to reduce potential experimenter effects and mimic a realistic online environment as suggested in prior work [72,76,78].

During the quiz, the SupportBot guides the user through the steps shown in Fig. 1. First, the bot displays a MCQ with four answer options, requesting the user to attempt the question by themselves (Step 1). Upon submitting their personal answer, the bot asks the user to rate how confident they are of the chosen answer from a scale of 1 -5 , with higher values indicating higher confidence (Step 2). After the user indicates their initial confidence level, the bot displays a list of group answers claiming to display how two 'peers' have answered the same question (Step 3). However, in reality there was only one real user in a single session and the 'peers' were simulated by two confederates of the research team, who provided answers to the quiz questions based on a predetermined script to ensure that one answer always secured a clear majority of votes, while also placing the user's initial answer in the majority as well as in the minority to avoid suspicion. We note that the notion of using confederates to maintain control over the majority-minority group formation was based on prior conformity literature $[3,4,8,45,79]$. Following the display of group answers, the bot requests the users to attempt the question again and indicate their confidence on the new answer (Steps 4 and 5), before moving to the next question (Step 6). The above process was repeated for all questions in the quiz, which allowed us to capture how the group feedback influenced users' decision to change or not change their initial answers. 


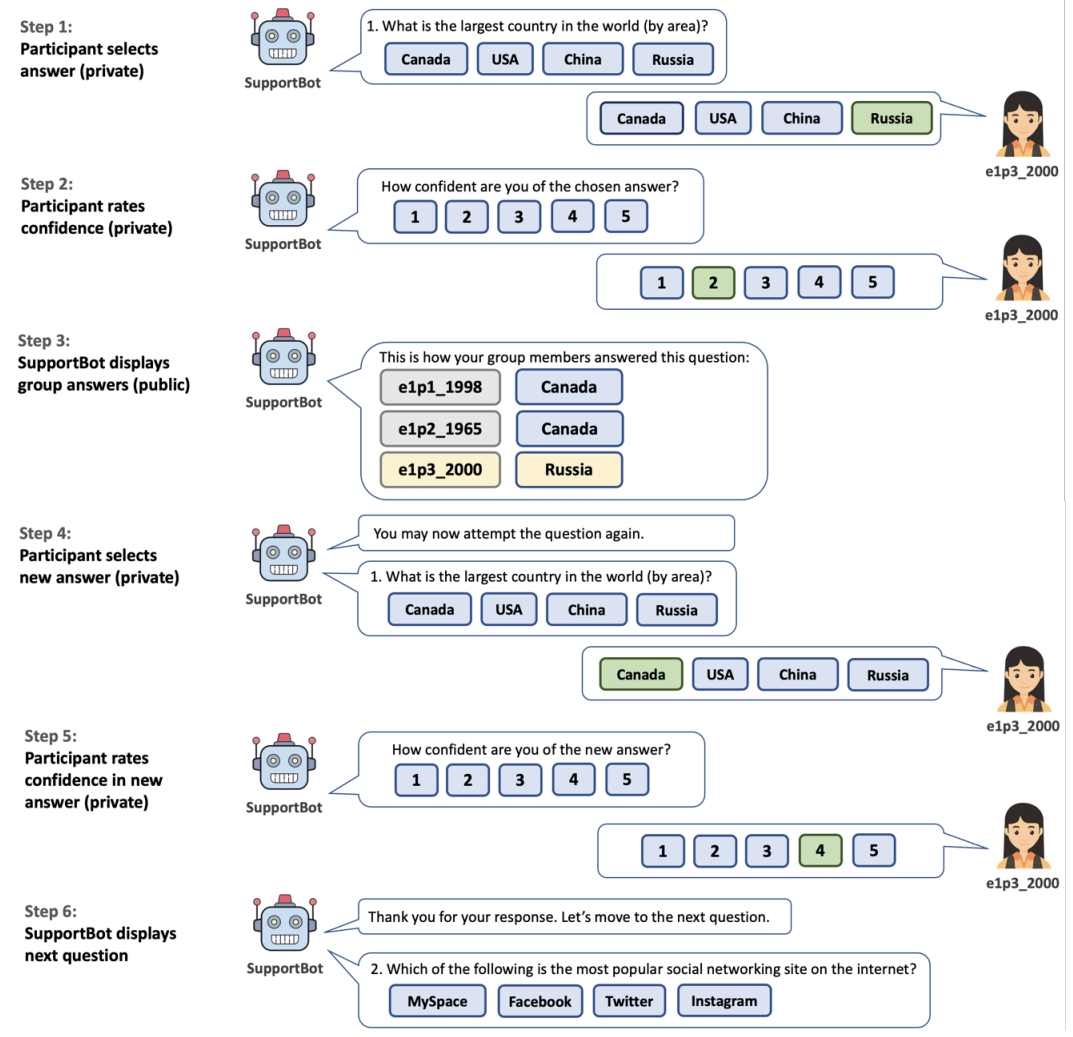

Fig. 1. Steps followed by a participant when answering the quiz questions.

\subsection{Age Group Compositions}

We decided to use an overall group size of three, which is the minimum group size required to simulate a clear majority against a minority of one (the user). Moreover, a group size of three has been established as sufficient to elicit conformity behaviour in prior studies $[3,4,11,27,63]$, which was appropriate for this experiment as our focus is not to determine the effect of group size on conformity behaviour.

As per the objectives of the experiment, to determine the effect of the opposing majority's age group composition on participants' conformity behaviour in stereotypically age-biased questions, we exposed users to the following age group compositions. We were interested in the group compositions where the user is challenged by an opposing majority that included at least one peer who does not belong to the same age group as the user as illustrated in Fig. 2.

(a) Young user challenged by two middle-aged peers.

(b) Young user challenged by one middle-aged peer and one young peer.

(c) Middle-aged user challenged by two young peers.

(d) Middle-aged user challenged by one young peer and one middle-aged peer. 


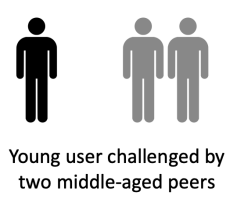

(a)

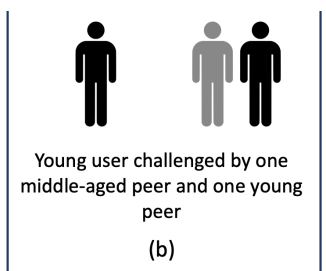

(b)
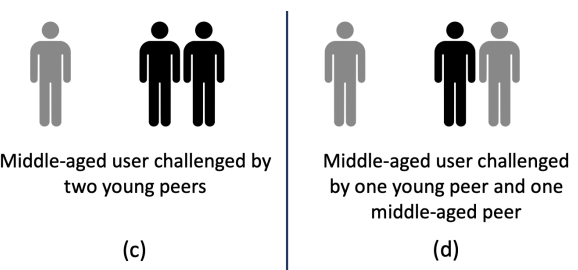
middle-aged peer

(d)

Fig. 2. Overview of age group compositions investigated in the study. The real participant/user is on the left and the simulated 'peers' are to the right. Black and grey avatars represent young users/peers and middle-aged users/peers respectively.

\subsection{Age Cues}

Determining how to indicate peer age to users realistically was a critical decision of this study. We decided against using real photographs of users, as photographs are rich in user cues other than age, and have been seen to elicit stereotypical perceptions of user gender, personality, and even their trustworthiness in online settings subsequently affecting their conformity behaviour $[32,62,75,77]$. Instead, we chose to represent users using textual usernames that included a unique user ID and their birth year (e.g. e1p3_2000 as shown in Fig. 1), to imply the age group users belonged to. Our decision was motivated by prior work that shows that users tend to include their birth year in online usernames in Twitter and other gaming platforms $[44,56]$. Furthermore, we chose the mode and median birth years of the young (1998 and 2000 respectively) and middle-aged (1965 and 1976 respectively) users recruited for the study, to be displayed alongside the 'peer' answers during the quiz (see Fig. 1 Step 3). Our intention behind this decision was to ensure that the fake peers represent the age distribution of the user cohort recruited for the study.

\subsection{Participants and Procedure}

We recruited participants using our university's online notice board where individuals willing to volunteer for the study were requested to complete a simple form providing their name, email address, self-described gender and birth year. We described that the study aims to investigate how individuals perform in online group quizzes, as the true purpose of the study could not be disclosed prior to the experiment as expected in conformity studies [67]. Next, we shortlisted potential participants based on their age group (young adults and middle-aged adults only), and contacted them through email to describe the experimental task and obtain their written consent. Out of the individuals who responded with their consent, we recruited a final sample $(N=32)$ that consisted of 16 middle-aged adults (Generation X; born between 1965 - 1980 as per [24]; $M=46.63, S D=5.84$ ) and 16 young adults (Generation Z; born between 1997 - 2002 as per [24]; $M=20.5$, $S D=1.84$ ), with an equal number of men and women from each age group. Moreover, our participants came from different educational and occupational backgrounds including Arts, Engineering, Science, Commerce, Physiotherapy, Nursing, Education and Public Health. Participants were then randomly assigned to the eligible experimental conditions (i.e. young adults were equally assigned to experimental conditions illustrated in Fig. 2 (a) and (b), and middle-aged adults were equally assigned to experimental conditions 
illustrated in Fig. 2 (c) and (d)), with an equal number of men and women to each experimental condition. Each participant was assigned to only one experimental condition.

The study was conducted entirely online, using Slack channels for each group session under the supervision of a researcher. Before the quiz, all participants received a link to join the designated Slack channel, using their unique username. Upon joining the Slack channel, the bot welcomed the participants, described the objective of the study and the experimental task, and informed the participants that they are connected with two 'peers' (simulated by confederates) to complete the quiz as a group. The bot also described that all participants are referred to using their usernames during the quiz, highlighting that the username is composed of a unique participant ID and the corresponding participant's birth year. Participants were then prompted to type "@SupportBot ready" once they have completed reading the instructions, upon which the quiz was initiated.

After completing the steps illustrated in Fig. 1 for each quiz question, the SupportBot automatically directed the participants to complete a post-test survey with three questions:

1. Did you experience an urge to change your initial answer after seeing the group answers? If yes, what factors influenced this behaviour?

2. How did you use the feedback received to answer the quiz questions?

3. Did you notice the age distribution of the group? If yes, how did this information affect your final answer?

After participants submitted brief, textual responses to the above questions, the bot thanked them, and explained the true objective of the study and the use of confederates to simulate peers. Participants were then given the opportunity to withdraw their participation and data collected during the study, if desired. No participant chose to do so.

On a different note, we emphasise that the research team includes members from Generation X and Z (age groups considered for participant recruitment) as well as from Generation Y (the age group in between the two age groups investigated in the study). Moreover, the experimental design was approved by the Ethics Committee of our university. The experiment lasted for approximately 30-40 minutes per participant, including briefing, completing the quiz, and the final post-test survey.

\subsection{Analysis of Survey Responses}

Two of the paper's authors individually coded the survey data following an inductive thematic analysis approach [12]. The emerging themes were then combined in an online spreadsheet before further discussion. These themes indicated perceived pressure from majority, a relationship between confidence in answer and conformity, and how inferring peer age through usernames led to age-stereotypical perceptions of peer competency. Next, the two authors virtually discussed and collaboratively agreed on the final themes: effectiveness of the manipulations used in the study to trigger age-related stereotypes (usernames and questions stereotypically perceived as age-biased), how participants' age, opposing majority's age group composition and perceived question type affected participants' conformity behaviour, and the effect of confidence in initial answer on subsequent conformity behaviour. Next, we present the main findings of our analysis. 
Age Cues and Stereotypical Question Types: During the quiz, participants were addressed using usernames which included a unique user ID and their birth year (e.g. e1p1_1972). The SupportBot also informed that the same naming convention is used to refer to their peers during the quiz (especially when displaying peer answers next to their usernames as shown in Fig. 1, Step 3). In the post-test survey, all participants described that they actively inferred peer age and the age group their peers belonged to (young vs. middle-aged adults), using usernames of peers. Hence, embedding birth years in usernames of the supposed peers was sufficient to trigger awareness of peer age; "I noticed that each of us is from quite different generations. One of them was born in 1965, another in 1976 and myself in 2000. My peers knew many other things in their era that I did not know, and vice versa" (P16, Gen Z).

Moreover, participants stereotypically categorised quiz questions as better known by young (i.e. social media \& latest technology) and middle-aged (i.e. 1980's history) adults, indicating that the question types we used in the quiz to trigger age-biased stereotypical perceptions were effective; "I trusted the older guy (born in 60s) when answering questions from his era (like the movie from the 80's), while I trusted the guy born in 1998 when answering questions like the most popular social media website" (P23, Gen Z).

Age-related Stereotypes: We further note that the awareness of peer age and assumptions of stereotypical question types significantly encouraged participants to stereotypically perceive peer competency in different questions. The majority of the participants described that they actively linked peer age with the stereotypically assumed era of the question, when deciding whether to trust peer answers or not; "Some questions are too new like social networking, technology and gaming. I think only young people would know these. But there were also questions that are old (1980s), for which I don't believe the younger generation would know the correct answer" (P10, Gen X) and "I will consider peer answers more if the question is something related to events or things of their generation" (P02, Gen Z).

Moreover, participants also highlighted how such (stereotypical) perceptions impacted their conformity behaviour. As noted by P25 - a middle-aged participant - they were more inclined to conform to younger majorities in questions perceived to be better known by younger generations; "A lot of the questions were based around technology and recent things. I thought younger people would know more about these topics, and if I didn't know the answer, I thought they would know better and I trusted them" (P25, Gen X). Similarly, young participants also claimed to prefer majorities with middle-aged peers for questions that they perceived to be familiar to older generations. Alternatively, for questions they perceived to be familiar to their own age group, they were less inclined to change their personal answer; "When the question is about history or requires knowledge related to many years ago, I preferred to change my answers so that they can be consistent with the older people's answer" (P05, Gen Z) and "The age distribution only affected my decisions in questions that might have been popular in the past as I was the youngest among the group" (P03, Gen Z).

Our data also provide evidence as to why participants felt encouraged to follow aforementioned stereotypically age-biased perceptions when deciding whether to conform or not. Participants rationalised that following the answers of the age group that is perceived to be more familiar of the question content, improved their chances of reaching the correct answer to the quiz questions - indicating the presence of informational conformity influences; "When the questions were related to an older time, for instance, 
1980's popular film, it makes sense to have a higher bet on a person from that era than someone younger. So age did convince me to believe that they might have chosen the right answer" (P24, Gen Z) and "I used the 90's person's answers for gaming questions, and if the 60's person had the same answer as me [a middle-aged participant] for history questions, I felt a little more confident that my answer may be correct" (P31, Gen X).

Initial Confidence: Our qualitative analysis also indicate that participants were more susceptible to conformity influences when they were unsure of their personal answers, whereas they were less likely to conform when they trusted their personal answers; "If I was not very confident in my answer, I would look at what the other two posted. If they both agreed on something different from me, I was likely to change to what they said. If I was reasonably confident, but others gave a different answer, I generally stuck to my answer, but my confidence was less" (P27, Gen X). Moreover, participants reiterated that the peer feedback was useful when they were unsure of their initial choice, to reach the correct answer to the quiz question (informational conformity); "For some questions I was really only guessing the answers, so answers from my teammates provided me with an answer which I hoped was more likely to be correct than my guess" (P27, Gen X).

\section{Results}

All 32 participants answered 30 multiple-choice questions (equally distributed among topics covering general knowledge, social media \& technology, and 1980's history) which resulted in a total of 960 responses. Moreover, simulated peer answers placed participants in minorities (peer answers unanimously challenged participant's answer) as well as in majorities (one or more peer answers supported the participant's answer) to avoid suspicion. As a result, participants found themselves in the group majority in 618 questions, and in a minority for the rest of the 342 questions. On that note, we emphasise that aim of this study is not to compare results between majority and minority groups, but rather to investigate the impact of opposing majority's age group composition on conformity behaviour, when answering stereotypically age-biased questions.

Upon seeing the group's answers for a question, participants indicated their final answer and confidence level, where they could:

(a) Change both their initial answer and confidence level.

(b) Change only their initial answer.

(c) Change only their confidence in answer.

(d) Make no change to either their initial answer or confidence level.

We note that all participants changed their opinion, confidence level, or both at least once during the study, resulting in a total of 481 changed responses (in majority $=263$, in minority $=218)$ with an average of 15.03 changes $(S D=5.62)$ per participant. Fig. 3 illustrates the distribution of the final responses (post-feedback), grouped by whether the participants' initial answer to the question placed them in a minority (minority responses), or a majority (majority responses). When placed in minorities, participants changed their initial answer with or without a change in confidence in $46.4 \%$ of the responses, changed only their confidence in $17.3 \%$ of the responses $(M=-0.31$ and $S D=1.44$ per response, indicating an overall reduction in confidence) and made no change to their initial answer 


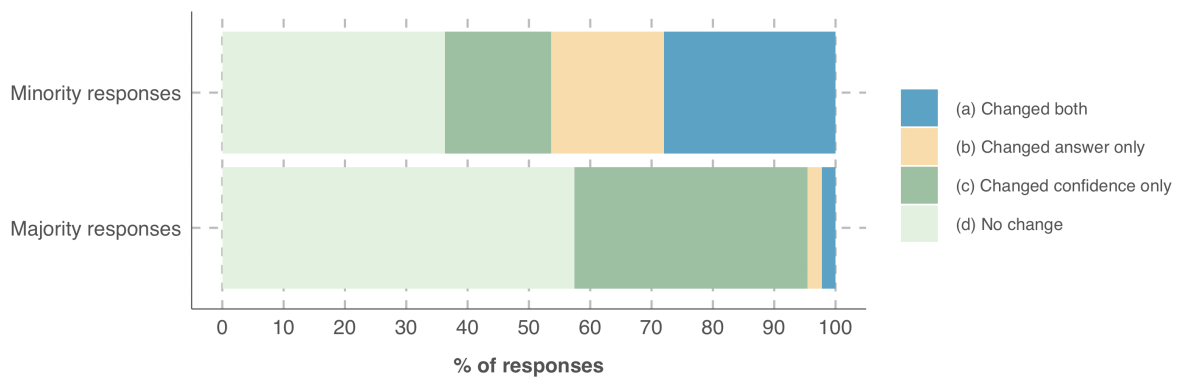

Fig. 3. Distribution of minority and majority responses across the four response types.

or confidence in the remaining $36.3 \%$ of the responses. Conversely, when participants found themselves in the group's majority they made either no change to their initial response or changed only their confidence level in the initial answer $(M=1.14$ and $S D=$ 1.03 per response, indicating an overall increase in confidence) in approximately $95.4 \%$ of the responses. Hence, our preliminary analysis indicates that participants were aware of their position in the group, and changed their answers post feedback, not randomly but due to the influence of the predictors we considered, confirming the validity of our results.

\subsection{Model Construction}

For the purposes of this study, we consider changing the initial answer option (with or without a change in initial confidence level) to that of the majority, as conformity behaviour. Our results show that 29 (out of 32) participants conformed at least once to the majority, resulting in a total of 97 conformity responses (conformity rate $=28.36 \%$ ), with an average of $3.03(S D=2.13)$ conformity responses per participant. We observe similar conformity rates in prior online conformity literature $[45,77,79]$.

We then investigated the impact of the following variables on the conformity behaviour of our participants. The predictor variables were chosen based on the study's objective of determining the effects of participant age, opposing majority's age group composition, and stereotypical question type on online conformity behaviour. For the statistical model we only considered the responses of participants when placed in a minority, as the dependent variable was determining conformity behaviour.

- PAge: Participant's age group. Values: Middle-aged (Generation X), Young (Generation Z).

- MajAgeGroup: Majority's age group. Values: Middle-aged (majority of two middle-aged peers), Young (majority of two young peers), Mixed (majority of one young and one middle-aged peer).

- QType: Stereotypically perceived question type. Values: Neutral (general knowledge), Young (social media \& latest technology) and Middle-Aged (1980's history).

- Initial confidence: Participant's initial confidence in their answer. Range: 1-5.

- Gender: Participant's self-disclosed gender. Values: Man, Woman.

- User ID: An unique identifier assigned to a given user during the quiz. 
We used the R package lme4 [6] to perform a generalised linear mixed-effects model (GLMM) analysis of the relationship between the aforementioned variables and participant conformity behaviour (binary variable: conformed or not). A GLMM (family = binomial, link = logit) supports the study's objective to identify potential main and/or interaction effects from multiple personal determinants of offline conformity (i.e., age, gender, self-confidence), in addition to the impact of stereotypical perceptions triggered by age-typed questions and majority's age group composition, on the outcome variable - conformity - which follows a non-normal distribution. We specified participant (User ID) as a random effect to account for individual differences in our model.

All statistically significant predictors included in the final model (following model selection through incremental addition of variables based on their predictive power) are shown in Table 2. We perform a likelihood ratio test with the null model [10] and find that our model is statistically significant $\left(\chi^{2}=107.56, p<0.001\right)$ and explains $38.21 \%$ of the variance in accuracy $\left(R=0.62, R^{2}=0.38\right)$. To ensure the validity of the model, we check for the existence of multicollinearity. Our predictors report a variance inflation factor between 1.16 and 2.71, well below the often-used threshold of 5 to detect multicollinearity [33].

We observe a statistically significant interaction effect between participant's age group, opposing majority's age group composition and stereotypical question type, on participants' conformity behaviour $(p<0.05)$. The model also notes that participants' confidence on initial answer indicates a statistically significant main effect on their conformity behaviour $(p<0.001)$. Our results do not indicate any other main or interaction effects from the variables considered. Next, we describe the above significant predictors in detail.

\begin{tabular}{lrrr}
\hline Predictor & \multicolumn{2}{c}{ Log OR P-value Effect size (d) } \\
\hline Initial confidence & $\mathbf{- 0 . 6 5}<\mathbf{0 . 0 0 1}$ & $\mathbf{- 0 . 3 5 9}$ \\
PAge (Middle-aged) : QType (Young) : MajAgeGroup (Young) & $\mathbf{2 . 7 0}<\mathbf{0 . 0 0 1}$ & $\mathbf{1 . 5 0 3}$ \\
PAge (Middle-aged) : QType (Middle-aged) : MajAgeGroup (Young) & $\mathbf{1 . 9 1}$ & $\mathbf{0 . 0 4}$ & $\mathbf{1 . 0 7 2}$ \\
PAge (Middle-aged) : QType (Neutral) : MajAgeGroup (Young) & 1.61 & 0.07 & 0.904 \\
PAge (Young) : QType (Middle-aged) : MajAgeGroup (Middle-aged) & $\mathbf{1 . 7 0}$ & $\mathbf{0 . 0 3}$ & $\mathbf{0 . 9 5 2}$ \\
PAge (Young) : QType (Young) : MajAgeGroup (Middle-aged) & 1.57 & 0.11 & 0.884 \\
PAge (Young) : QType (Neutral) : MajAgeGroup (Middle-aged) & 0.97 & 0.31 & 0.551 \\
PAge (Middle-aged) : QType (Middle-aged) : MajAgeGroup (Mixed) & -0.75 & 0.39 & -0.406 \\
PAge (Middle-aged) : QType (Young) : MajAgeGroup (Mixed) & 0.42 & 0.59 & 0.238 \\
PAge (Middle-aged) : QType (Neutral) : MajAgeGroup (Mixed) & -0.87 & 0.27 & -0.473 \\
PAge (Young) : QType (Middle-aged) : MajAgeGroup (Mixed) & -0.33 & 0.66 & -0.181 \\
PAge (Young) : QType (Neutral) : MajAgeGroup (Mixed) & -0.77 & 0.30 & -0.418 \\
\hline
\end{tabular}

Table 2. Effect of predictors on participant conformity. Statistically significant main effects and interactions $(p<0.05)$ are in bold. The effect sizes are presented as Cohen's $d$ values derived based on relevant log odds ratio [58]. The sign of the effect size or $d(+/-)$ denotes the direction of the relationship between the predictor and conformity behaviour. The absolute size of $d$ indicates the magnitude of the effect; $\mathrm{d}=0.2$ (small), $\mathrm{d}=0.5$ (medium) and $\mathrm{d}=0.8$ (large) [18].

\subsection{Participant's Age Group, Opposing Majority's Age Group Composition and Stereotypical Question Type}

We note that participants' tendency to conform to the group majority, was significantly influenced by their age group, opposing majority's age group composition and the stereotypically perceived question type as illustrated in Fig. 4 (a) and (b). The two plots visualise the density of the likelihood of conformity (y-axis) for young \& middle-aged 
participants, when challenged by middle-aged and young majorities respectively (x-axis), across the three question types. We calculated the likelihood of conformity for each participant in each of the three question types, as the ratio between the number of conformity responses and the total number of minority responses in the relevant question type.

(a) Young participants against middle-aged majorities

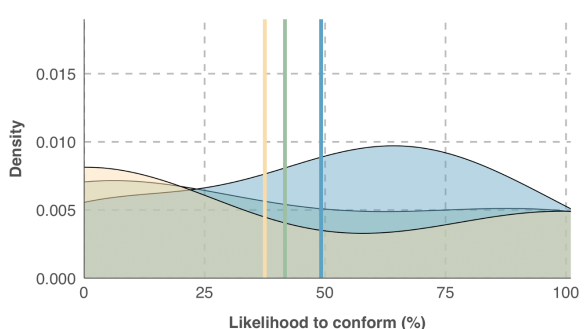

Likelihood to conform $(\%)$ (b) Middle-aged participants against young majorities

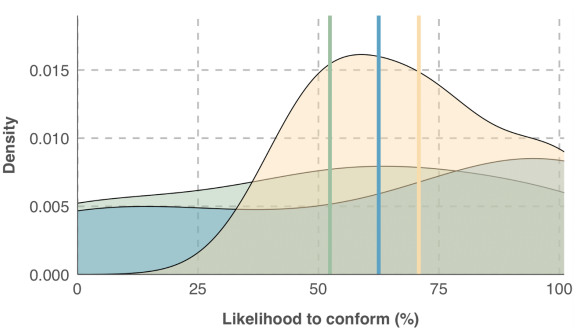

Likelihood to conform (\%)

General Knowledge (Neutral)

1980 's History (Middle-aged)

Social Media \& Technology (Young)

Fig. 4. (a) Likelihood of young participants conforming to middle-aged majorities in different question types, and (b) Likelihood of middle-aged participants conforming to young majorities in different question types. The three curves relate to the three question types. Vertical lines indicate average conformity rates for each question type.

Fig. 4(a) indicates that young participants were significantly more inclined to conform to majorities with all middle-aged peers for questions stereotypically perceived to be well-known by middle-aged adults (1980's history) than in other question types $(d=0.952$, large effect size), with their average conformity rates are at $49.17 \%, 41.67 \%$ and $37.50 \%$ for 1980 's history (middle-aged), general knowledge (neutral) and social media \& technology (young) questions respectively. On the other hand, middle-aged participants were seen to conform to majorities with all-young peers, in questions which are stereotypically age-biased in comparison neutral questions. This behaviour was more dominant in questions which are sterotypically perceived to be well-known by young adults $(d=1.503$, large effect size), than in questions which are stereotypically perceived to be well-known by middle-aged adults $(d=1.072$, large effect size). This interaction effect is illustrated in Fig. 4(b), which marks the average conformity rates of middle-aged participants at $70.83 \%, 62.50 \%$ and $52.38 \%$ for social media \& technology (young), 1980 's history (middle-aged) and general knowledge (neutral) questions respectively. Moreover, conformity behaviour of both young and middle-aged participants were not significantly affected by mixed majorities, in any of the question types.

\subsection{Initial Confidence}

The statistically significant main effect from participants' initial confidence on their subsequent conformity behaviour $(p<0.001)$ implies that all participants were less likely to conform to the majority, when they were confident of their initial answer - regardless the majority's age group composition or the stereotypically perceived question type 
( $d=-0.359$, small to medium effect size). We illustrate this in Fig. 5 , where we analysed participants' self-reported initial confidence levels across both non-conforming and conforming responses using box-plots. We note that while the range of self-reported confidence levels for both response types range from 1-5, the median values for nonconforming and conforming responses are at $3(M=2.89, S D=1.31)$ and $2(M=2.08$, $S D=1.09)$ respectively, reiterating that lower initial confidence values are more likely to result in conforming behaviour.

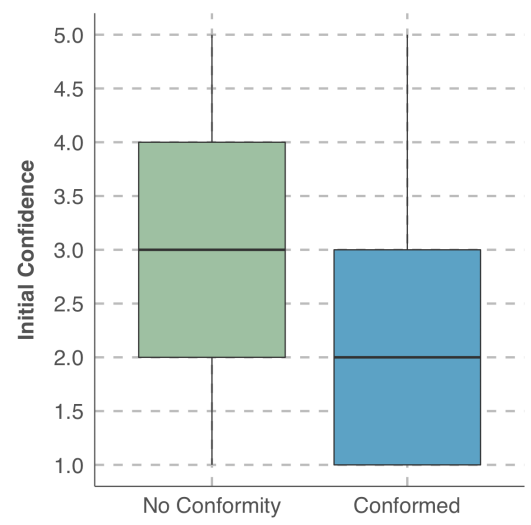

Fig. 5. Participants' initial confidence across non-conforming and conforming minority responses.

\section{Discussion}

Currently, our understanding of online social conformity is primarily based on its contextual determinants - i.e., group size [61,78,39,77], social presence [45,79], and task objectivity $[45,61,78,79]$. However, as online group platforms are becoming increasingly personal (e.g., social media, online support groups $[7,5,15,28,51,60,73])$, it is vital to quantify implications of personal factors such as age and gender on social conformity. Moreover, such personal factors have also been seen to trigger stereotypical perceptions of peer competency among individuals in offline groups, further increasing their susceptibility to stereotypical conformity influences $[14,25,43]$. However, it is unclear if the above observations would prevail in online groups that lack direct face-to-face interactions, and operate with minimum user cues [54]. Hence, this study takes an initial step towards investigating effects of one such personal factor - age - on online social conformity across three aspects: age group of the participant, age group composition of the opposing group majority, and stereotypically perceived question type.

\subsection{Online Social Conformity}

We note an overall conformity rate of $28.36 \%$ - which is similar to conformity rates observed in prior work that investigated effects of contextual conformity determinants $[45,77,79]$. Furthermore, our results indicate a statistically significant main effect 
of participants' initial confidence in personal answer, and an interaction effect from the three aspects of age considered in the study, on the conformity behaviour of our participants. Hence, by controlling for contextual determinants such as majority group size (majority was always two), social presence (low social presence, with minimum users cues and interactivity) and task objectivity (all questions were objective), this study revealed implications of personal conformity determinants - which we discuss next.

Initial Confidence: Participants' confidence on initial judgements has been previously reported to influence their susceptibility to conformity in online groups $[8,47,49,77,78,79]$. Similar to prior observations, we also show that participants are significantly less motivated to change their answer to the majority's when confident of their personal answer, whereas when they are unsure of their initial selection they readily change their final answer to reflect the majority's selection. We further emphasise that this effect persist, regardless of the other factors considered in the study (participant's age, opposing majority's age group composition, and question type). Moreover, in the post-test survey participants described that conforming to the majority when unsure of the their initial answer was a mechanism employed to 'correctly' answer the quiz questions - confirming the existence of informational influences as implied in prior studies $[77,78,79,80]$.

Age and related Stereotypes: While our results did not indicate main effects from the three aspects of age included in the study, we note that participants' age group (young adult or Generation Z vs. middle-aged adult or Generation X) interacted with the opposing majority's age group composition (all young peers, all middle-aged peers, or a mix of young and middle-aged peers) and the stereotypically perceived question type (social media \& latest technology favouring Generation Z, 1980's history favouring Generation X, and general knowledge that do not favour an age group), in determining their conformity behaviour. More specifically, young adults were swayed by an opposing majority with all middle-aged peers, strictly in questions stereotypically perceived to be better known to middle-aged adults (1980's history), whereas middle-aged adults readily conformed to opposing majorities that included all young peers, in questions stereotypically perceived to be well known to young adults (social media \& latest technology).

Therefore, while our study did not replicate observations of offline literature where older adults are portrayed as more susceptible to conformity influences than their younger counterparts $[41,42,43]$, our findings show that stereotypical perceptions that participants derived with regard to peer competency using peer age as an indicator, substantially influenced their online conformity behaviour. Our qualitative analysis confirms that the above perceptions of peer competency were based on popular stereotypes that portray young adults (or the Generation Z) as "digital natives" [24,71], and middle-aged adults (or the Generation X) to possess more "practical knowledge and life experience" $[13,36,40,70]$. Consequently, participants believed that following the age group that is stereotypically perceived to be more knowledgeable about certain age-biased topics, improved their chances of being 'correct' - reiterating effects of informational influences that have been previously observed with regard to gender stereotypical perceptions of peer competency [49,77]. Moreover, the fact that both young and middle-aged participants showed no motivation to conform to mixed majorities in any of the question types, suggests that participants were not concerned about being singled out against a unanimous majority, and hence were not as susceptible to normative conformity influences. 
Furthermore, we highlight that prior work that investigated young people's actual use of technology do not identify them as expert users in the matter [64]. Moreover, literature also note that other factors such as education level of young people significantly affect their expertise in technology [1]. Therefore, stereotypical conformity observed in this study, where young adults were stereotyped as more competent in social media \& technology entirely based on their assumed age, is not always reliable and is unlikely to result in 'correct' answers as expected. Furthermore, other studies also indicate that age-biased stereotypes often disadvantage older adults who are perceived as less reliable and trustworthy than their younger counterparts, in online group settings $[57,30,34]$. Therefore, our findings coupled with prior evidence in literature, urge the re-evaluation of online group platform design, to mitigate undesirable effects of age-stereotypical online conformity behaviour i.e., conforming to incorrect majorities and prejudice against people from different age generations.

\subsection{Design Implications}

This study presents interesting findings with regard to the use of user cues in online groups. We note that despite its minimalist nature, textual usernames that indicated the birth year of the corresponding peer were sufficient to trigger stereotypical perceptions of peer competency, in both young and middle aged participants - which also subsequently determined their conformity behaviour. Hence, our findings imply that individuals are receptive to the simplest user cues in online groups and often use them to derive stereotypical perceptions of their online peers.

Moreover, it is likely that the effects of age and related stereotypes observed in this study would be further heightened in real online group settings that use richer user representations such as real photographs (e.g., social media, online forums $[55,68]$ ) and highly anthropomorphic (human-like) avatars (e.g., gaming platforms, virtual worlds $[34,50])$. Therefore, we urge designers of online group platforms to reconsider if including user cues is of value to the core purpose of the platform to minimise susceptibility of users to unwanted social pressures. For instance, user age holds important information in online dating websites or social media, but may not be useful in an e-commerce platform. Thus, age-related user cues should only be embedded in platform design only if they are considered value-adding.

Furthermore, we encourage the use of online user representations that are devoid of explicit age-related information - i.e., site specific avatars used by Slack, animal avatars used by Google, identicons used by GitHub etc. - especially in contexts where age stereotypical perceptions of peer competency could trigger conformity behaviour as observed in this study. We argue that using age-neutral user representations in platform design can minimise the occurrence of age-stereotypes that have been observed to trigger prejudice against certain age groups in prior work $[57,30,34]$.

Alternatively, we encourage future studies to explore the possibility of mitigating detrimental effects of age-related stereotypes through alternative user representations, and by displaying user competency through platform specific indicators (e.g., skill assessment tests in LinkedIn [38], badges used in Stack Overflow [31]) - thereby minimising opportunity for stereotypical perceptions of user competency to manifest. 


\subsection{Limitations}

We note the following limitations of our study. First, our findings on the effects of age and related stereotypes on online conformity behaviour are specifically with regard to young and middle-aged adults. Hence, further work is required to investigate how age and related stereotypes impact conformity behaviour in other age groups (such as adolescents and older adults). Furthermore, while the sample size used for the study was sufficient to elicit statistically significant effects from age-stereotypical perceptions on online conformity behaviour, further work is required to replicate our findings in larger sample sizes. Moreover, as the study's primary focus was on understanding the impact of personal determinants such as user age and their susceptibility to stereotypes on online conformity behaviour, we did not investigate how the above personal factors manifest alongside popular contextual determinants such as group size, task objectivity and social presence. Therefore, we note that our work is an initial step towards quantifying effects of personal determinants on online conformity, and that future work can extend this work to investigate combined effects of both personal and contextual determinants on online conformity behaviour.

\section{Conclusion}

While age has been identified as a critical conformity determinant in offline groups, its effects on online conformity remained unclear. Hence, this study investigates effects of age and related stereotypes on susceptibility to conformity influences in young and middleaged adults, as they complete stereotypically age-biased tasks in an online chatroom. Our results indicate that in the absence of explicit information of peer competency, both young and middle-aged adults stereotypically perceived competency of their online peers based on assumed peer age (indicated through usernames of peers) - establishing the existence of age stereotypes in online groups. Furthermore, such stereotypical perceptions were also seen to influence the conformity behaviour of our participants. We note that both young and middle-aged participants conformed to their older or younger counterparts, when attempting tasks that are stereotypically perceived to be well known to the respective age group. Our qualitative data provides further evidence that in the presence of user cues that indicate peer age, the effect of traditional informational influences on online conformity was further heightened by age-related stereotypical perceptions. We discuss how our findings encourage designers of online group settings to carefully reconsider if embedding user cues in platform design is value-adding from the perspective of the users as well as the platform. Furthermore, we promote the use of online user representations that are devoid of age cues (e.g., identicons or site-specific ageneutral avatars instead of anthropomorphic (human-like) avatars or real photographs of users) - especially in contexts where age cues could trigger age-stereotypical perceptions of peer competency. In conclusion, our results highlight the need for exploring alternative online user representations and platform specific indicators of peer competency to minimise detrimental implications of stereotypical conformity in online groups. 


\section{References}

1. Akçayır, M., Dündar, H., Akçayır, G.: What makes you a digital native? is it enough to be born after 1980? Computers in Human Behavior 60, 435-440 (2016)

2. Araújo, C.S., Meira, W., Almeida, V.: Identifying stereotypes in the online perception of physical attractiveness. In: Spiro, E., Ahn, Y.Y. (eds.) Social Informatics. pp. 419-437. Springer International Publishing, Cham (2016)

3. Asch, S.E.: Groups, leadership and men, chapter: Effects of group pressure upon the modification and distortion of judgements, pp. 177-190. Carnegie Press, Oxford, England (1951)

4. Asch, S.E.: Opinions and social pressure. Scientific American 193(5), 31-35 (1955)

5. Barkhuus, L., Tashiro, J.: Student socialization in the age of facebook. In: Proceedings of the SIGCHI Conference on Human Factors in Computing Systems. pp. 133-142. CHI '10, ACM, New York, NY, USA (2010)

6. Bates, D., Mächler, M., Bolker, B., Walker, S.: Fitting linear mixed-effects models using lme4. Journal of Statistical Software 67(1), 1-48 (2015)

7. Baym, N.K.: Personal connections in the digital age. John Wiley \& Sons (2015)

8. Beran, T., Drefs, M., Kaba, A., Al Baz, N., Al Harbi, N.: Conformity of responses among graduate students in an online environment. The Internet and Higher Education 25, 63-69 (2015)

9. Blake, R.R., Helson, H., Mouton, J.S.: The generality of conformity behavior as a function of factual anchorage. difficulty of task, and amount of social pressure. Journal of Personality 25(3), 294-305 (1957)

10. Bolker, B.M., Brooks, M.E., Clark, C.J., Geange, S.W., Poulsen, J.R., Stevens, M.H.H., White, J.S.S.: Generalized linear mixed models: a practical guide for ecology and evolution. Trends in Ecology \& Evolution 24(3), 127-135 (2009)

11. Bond, R.: Group size and conformity. Group processes \& intergroup relations 8(4), 331-354 (2005)

12. Braun, V., Clarke, V.: Using thematic analysis in psychology. Qualitative Research in Psychology 3(2), 77-101 (2006)

13. Broady, T., Chan, A., Caputi, P.: Comparison of older and younger adults' attitudes towards and abilities with computers: Implications for training and learning. British Journal of Educational Technology 41(3), 473-485 (2010)

14. Brody, G.H., Stoneman, Z.: Peer imitation: An examination of status and competence hypotheses. The Journal of genetic psychology 146(2), 161-170 (1985)

15. Brzozowski, M.J., Adams, P., Chi, E.H.: Google+ communities as plazas and topic boards. In: Proceedings of the 33rd Annual ACM Conference on Human Factors in Computing Systems. pp. 3779-3788. CHI '15, ACM, New York, NY, USA (2015)

16. Christofides, E., Islam, T., Desmarais, S.: Gender stereotyping over instant messenger: The effects of gender and context. Computers in Human Behavior 25(4), 897-901 (2009)

17. Cinnirella, M., Green, B.: Does 'cyber-conformity' vary cross-culturally? exploring the effect of culture and communication medium on social conformity. Computers in Human Behavior 23(4), 2011-2025 (2007)

18. Cohen, J.: Statistical power analysis for the behavioral sciences. Lawrence Erlbaum Associates (1988)

19. Colliander, J.: This is fake news: Investigating the role of conformity to other users' views when commenting on and spreading disinformation in social media. Computers in Human Behavior 97, 202-215 (2019)

20. Conaway, W., Bethune, S.: Implicit bias and first name stereotypes: What are the implications for online instruction?. Online Learning 19(3), 162-178 (2015)

21. Costanzo, P.R., Reitan, H.T., Shaw, M.E.: Conformity as a function of experimentally induced minority and majority competence. Psychonomic Science 10(10), 329-330 (1968)

22. Costanzo, P.R., Shaw, M.E.: Conformity as a function of age level. Child development pp. 967-975 (1966) 
23. Deutsch, M., Gerard, H.B.: A study of normative and informational social influences upon individual judgment. The Journal of Abnormal and Social Psychology 51(3), 629-636 (1955)

24. Dimock, M.: Defining generations: Where millennials end and generation z begins. Pew Research Center 17(1), 1-7 (2019)

25. Fiske, S.T.: Stereotyping, prejudice, and discrimination. The handbook of social psychology 2(4), 357-411 (1998)

26. Fleiss, J.L., Levin, B., Paik, M.C.: Statistical methods for rates and proportions. john wiley \& sons (2013)

27. Gerard, H.B., Wilhelmy, R.A., Conolley, E.S.: Conformity and group size. Journal of Personality and Social Psychology 8(1, Pt.1), 79-82 (1968)

28. Goncalves, J., Kostakos, V., Venkatanathan, J.: Narrowcasting in social media: Effects and perceptions. In: 2013 IEEE/ACM International Conference on Advances in Social Networks Analysis and Mining (ASONAM 2013). pp. 502-509. IEEE (2013)

29. Goncalves, J., Liu, Y., Xiao, B., Chaudhry, S., Hosio, S., Kostakos, V.: Increasing the reach of government social media: A case study in modeling government-citizen interaction on facebook. Policy \& Internet 7(1), 80-102 (2015)

30. Gonzalez, L., Loureiro, Y.K.: When can a photo increase credit? the impact of lender and borrower profiles on online peer-to-peer loans. Journal of Behavioral and Experimental Finance 2, 44-58 (2014)

31. Gwosdz, M.M.: Stack overflow badges explained (April 2021), https://stackoverflow. blog/2021/04/12/stack-overflow-badges-explained, [Online; accessed 29-May-2021]

32. Haferkamp, N., Eimler, S.C., Papadakis, A.M., Kruck, J.V.: Men are from mars, women are from venus? examining gender differences in self-presentation on social networking sites. Cyberpsychology, Behavior, and Social Networking 15(2), 91-98 (2012)

33. Hair, J.F., Black, W.C., Babin, B.J., Anderson, R.E., Tatham, R.: Multivariate Data Analysis. Pearson, New Jersey, NJ, USA (2010)

34. Hasler, B.S., Tuchman, P., Friedman, D.: Virtual research assistants: Replacing human interviewers by automated avatars in virtual worlds. Computers in Human Behavior 29(4), 1608-1616 (2013)

35. Hullman, J., Adar, E., Shah, P.: The impact of social information on visual judgments. In: Proceedings of the SIGCHI Conference on Human Factors in Computing Systems. pp. 1461-1470. CHI '11, ACM, New York, NY, USA (2011)

36. Hummert, M.L., Garstka, T.A., Shaner, J.L., Strahm, S.: Stereotypes of the elderly held by young, middle-aged, and elderly adults. Journal of Gerontology 49(5), P240-P249 (1994)

37. Insko, C.A., Smith, R.H., Alicke, M.D., Wade, J., Taylor, S.: Conformity and group size: The concern with being right and the concern with being liked. Personality and Social Psychology Bulletin 11(1), 41-50 (1985)

38. Jersin, J.: Announcing skill assessments to help you showcase your skills (September 2019), https://blog.linkedin.com/2019/september/17/ announcing-skill-assessments-to-help-you-showcase-your-skills, [Online; accessed 29-May-2021]

39. Joinson, A.N.: Looking at, looking up or keeping up with people?: Motives and use of facebook. In: Proceedings of the SIGCHI Conference on Human Factors in Computing Systems. pp. 1027-1036. CHI '08, ACM, New York, NY, USA (2008)

40. Kessler, E.M., Rakoczy, K., Staudinger, U.M.: The portrayal of older people in prime time television series: The match with gerontological evidence. Ageing and society 24(4), $531-552$ (2004)

41. Klein, R.L.: Age, sex, and task difficulty as predictors of social conformity. Journal of Gerontology 27(2), 229-236 (1972)

42. Klein, R.L., Birren, J.E.: Age differences in social conformity on a task of auditory signal detection. In: Proceedings of the Annual Convention of the American Psychological Association. American Psychological Association (1972) 
43. Klein, R.L., Birren, J.E.: Age, perceived self-competence and conformity: A partial explanation. In: Proceedings of the Annual Convention of the American Psychological Association. American Psychological Association (1973)

44. Kokkinakis, A.V., Lin, J., Pavlas, D., Wade, A.R.: What's in a name? ages and names predict the valence of social interactions in a massive online game. Computers in Human behavior 55, 605-613 (2016)

45. Laporte, L., van Nimwegen, C., Uyttendaele, A.J.: Do people say what they think: Social conformity behavior in varying degrees of online social presence. In: Proceedings of the 6th Nordic Conference on Human-Computer Interaction: Extending Boundaries. pp. 305-314. NordiCHI '10, ACM, New York, NY, USA (2010)

46. Lee, E.J.: Effects of 'gender' of the computer on informational social influence: the moderating role of task type. International Journal of Human-Computer Studies 58(4), 347-362 (2003)

47. Lee, E.J.: Effects of gendered character representation on person perception and informational social influence in computer-mediated communication. Computers in Human Behavior 20(6), 779-799 (2004)

48. Lee, E.J.: When and how does depersonalization increase conformity to group norms in computer-mediated communication? Communication Research 33(6), 423-447 (2006)

49. Lee, E.J.: Wired for gender: Experientiality and gender-stereotyping in computer-mediated communication. Media Psychology 10(2), 182-210 (2007)

50. Lee, Y.H., Xiao, M., Wells, R.H.: The effects of avatars' age on older adults' self-disclosure and trust. Cyberpsychology, Behavior, and Social Networking 21(3), 173-178 (2018)

51. Liu, Y., Venkatanathan, J., Goncalves, J., Karapanos, E., Kostakos, V.: Modeling what friendship patterns on facebook reveal about personality and social capital. ACM Transactions on Computer-Human Interaction (TOCHI) 21(3), 1-20 (2014)

52. Maruyama, M., Robertson, S.P., Douglas, S., Raine, R., Semaan, B.: Social watching a civic broadcast: Understanding the effects of positive feedback and other users' opinions. In: Proceedings of the 2017 ACM Conference on Computer Supported Cooperative Work and Social Computing. pp. 794-807 (2017)

53. Maruyama, M.T., Robertson, S.P., Douglas, S.K., Semaan, B.C., Faucett, H.A.: Hybrid media consumption: How tweeting during a televised political debate influences the vote decision. In: Proceedings of the 17th ACM conference on Computer supported cooperative work \& social computing. pp. 1422-1432 (2014)

54. McKenna, K.Y., Green, A.S.: Virtual group dynamics. Group Dynamics: Theory, Research, and Practice 6(1), 116-127 (2002)

55. Nosko, A., Wood, E., Molema, S.: All about me: Disclosure in online social networking profiles: The case of facebook. Computers in human behavior 26(3), 406-418 (2010)

56. Olivier, J.: Twitter usernames: Exploring the nature of online south african nicknames. Nomina Africana 28(2), 51-74 (2014)

57. Pak, R., McLaughlin, A.C., Bass, B.: A multi-level analysis of the effects of age and gender stereotypes on trust in anthropomorphic technology by younger and older adults. Ergonomics 57(9), 1277-1289 (2014)

58. Polanin, J.R., Snilstveit, B.: Converting between effect sizes. Campbell Systematic Reviews 12(1), 1-13 (2016)

59. Posthuma, R.A., Campion, M.A.: Age stereotypes in the workplace: Common stereotypes, moderators, and future research directions. Journal of management 35(1), 158-188 (2009)

60. Reynolds, B., Venkatanathan, J., Gonçalves, J., Kostakos, V.: Sharing ephemeral information in online social networks: privacy perceptions and behaviours. In: IFIP Conference on Human-Computer Interaction. pp. 204-215. Springer (2011)

61. Rosander, M., Eriksson, O.: Conformity on the internet-the role of task difficulty and gender differences. Computers in Human Behavior 28(5), 1587-1595 (2012)

62. Rose, J., Mackey-Kallis, S., Shyles, L., Barry, K., Biagini, D., Hart, C., Jack, L.: Face it: The impact of gender on social media images. Communication Quarterly 60(5), 588-607 (2012) 
63. Rosenberg, L.: Group size, prior experience, and conformity. The Journal of Abnormal and Social Psychology 63(2), 436-437 (1961)

64. Selwyn, N.: The digital native - myth and reality. Aslib proceedings: New information perspectives 61(4), 364-379 (2009)

65. Sharma, E., De Choudhury, M.: Mental health support and its relationship to linguistic accommodation in online communities. In: Proceedings of the $2018 \mathrm{CHI}$ conference on human factors in computing systems. pp. 1-13 (2018)

66. Short, J., Williams, E., Christie, B.: The social psychology of telecommunications. John Wiley \& Sons (1976)

67. Stang, D.J.: Ineffective deception in conformity research: Some causes and consequences. European Journal of Social Psychology 6(3), 353-367 (1976)

68. Strano, M.M.: User descriptions and interpretations of self-presentation through facebook profile images. Cyberpsychology: journal of psychosocial research on cyberspace 2(2) (2008)

69. Sukumaran, A., Vezich, S., McHugh, M., Nass, C.: Normative influences on thoughtful online participation. In: Proceedings of the SIGCHI conference on human factors in computing systems. pp. 3401-3410 (2011)

70. Thomas, M.: Deconstructing digital natives: Young people, technology, and the new literacies. Taylor \& Francis (2011)

71. Turner, A.: Generation z: Technology and social interest. The journal of individual Psychology 71(2), 103-113 (2015)

72. Van Berkel, N., Goncalves, J., Hettiachchi, D., Wijenayake, S., Kelly, R.M., Kostakos, V.: Crowdsourcing perceptions of fair predictors for machine learning: a recidivism case study. Proceedings of the ACM on Human-Computer Interaction 3(CSCW), 1-21 (2019)

73. Venkatanathan, J., Karapanos, E., Kostakos, V., Gonçalves, J.: Network, personality and social capital. In: Proceedings of the 4th annual ACM web science conference. pp. 326-329 (2012)

74. Walker, M.B., Andrade, M.G.: Conformity in the asch task as a function of age. The Journal of social psychology 136(3), 367-372 (1996)

75. Walker, M., Vetter, T.: Changing the personality of a face: Perceived big two and big five personality factors modeled in real photographs. Journal of personality and social psychology 110(4), 609-624 (2016)

76. Wijenayake, S., van Berkel, N., Goncalves, J.: Bots for research: Minimising the experimenter effect. In: International Workshop on Detection and Design for Cognitive Biases in People and Computing Systems. CHI'20 Workshop, ACM (2020)

77. Wijenayake, S., van Berkel, N., Kostakos, V., Goncalves, J.: Measuring the effects of gender on online social conformity. Proceedings of the ACM on Human-Computer Interaction 3(CSCW), 1-24 (2019)

78. Wijenayake, S., van Berkel, N., Kostakos, V., Goncalves, J.: Impact of contextual and personal determinants on online social conformity. Computers in Human Behavior 108(106302), 1-11 (2020)

79. Wijenayake, S., van Berkel, N., Kostakos, V., Goncalves, J.: Quantifying the effect of social presence on online social conformity. Proceedings of the ACM on Human-Computer Interaction 4(CSCW1), 1-22 (2020)

80. Wijenayake, S., Hettiachchi, D., Hosio, S., Kostakos, V., Goncalves, J.: Effect of conformity on perceived trustworthiness of news in social media. IEEE Internet Computing 25(1), 12-19 (2021)

81. Winter, S., Brückner, C., Krämer, N.C.: They came, they liked, they commented: Social influence on facebook news channels. Cyberpsychology, Behavior, and Social Networking 18(8), 431-436 (2015)

82. Zhu, H., Huberman, B., Luon, Y.: To switch or not to switch: Understanding social influence in online choices. In: Proceedings of the SIGCHI Conference on Human Factors in Computing Systems. pp. 2257-2266. CHI '12, ACM, New York, NY, USA (2012) 


\section{Chapter 6}

\section{Gender as a Determinant of Conformity}

\subsection{Introduction}

Conformity literature in physical groups has shown significant gender differences in how people respond to conformity pressures - with women displaying more conformity behaviour than men in group settings $[36,52,98,148]$. Conversely, recent studies that account for user gender as a conformity determinant in online groups do not show significant gender differences $[139,188]$ - which is also confirmed by our results in Articles I \& II. However, gender stereotypical perceptions of a partner's competency on stereotypically gendered tasks have been found to determine user conformity behaviour in online dyadic interactions [102, 105].

This chapter conducts a thorough analysis into the effects of gender on online conformity, alongside other contextual and personal conformity determinants such as majority-minority group size and self-confidence. Similar to the experimental design of Article II, we investigate how user gender (man/woman), gender group composition of peers challenging and supporting user responses (e.g. an opposing majority with more women than men in the presence of a minority with more men than women), and the stereotypically perceived gender of task (masculine, feminine, and neutral) impact conformity behaviour in users who complete an online MCQ quiz together. Additionally, we used two popular gender cues - stereotypically gendered names and silhouette avatars - to represent supposed "peers" on the online quizzing platform, to analyse whether the strength of gender-stereotypical perceptions vary across different user representations.

Our results show that both men and women conform to majorities that have more stereotypically masculine user representations (names or avatars) in stereotypically masculine questions. Similarly, they conform to majorities with more stereotypically feminine user representations (names or avatars) in stereotypically feminine questions. This effect is stronger when using stereotypically gendered avatars as opposed to names. We further note significant effects from the majority-minority group size difference, and self-confidence which are inline with our findings in Articles I \& II. Furthermore, in the presence of stereotypically gendered user cues, conformity leads to more incorrect answers in stereotypically masculine and feminine questions, than in neutral questions.

Informed by our findings, we urge designers to re-evaluate the use of stereotypical gender cues in online platform designs and encourage using representations devoid of gender cues (e.g., identicons or site-specific avatars) - especially in situations where gender-stereotypical conformity can lead to adverse outcomes. More details of this study can be found in the attached publication, Article III. 


\subsection{Article III}

Copyright is held by the authors. Publication rights licensed to ACM. This is the authors' version of the work. It is posted here for your personal use. Not for redistribution. The definitive Version of Record was published in:

Wijenayake, S., van Berkel, N., Kostakos, V., Goncalves, J. (2019). Measuring the Effects of Gender on Online Social Conformity. Proceedings of the ACM on Human-Computer Interaction, 3(CSCW), 1-24. https://doi.org/10.1145/3359247.

Ethics ID: 1853038, The University of Melbourne Human Ethics Advisory Group. 


\title{
Measuring the Effects of Gender on Online Social Conformity
}

\author{
SENURI WIJENAYAKE, The University of Melbourne, Australia \\ NIELS VAN BERKEL, University College London, UK \\ VASSILIS KOSTAKOS, The University of Melbourne, Australia \\ JORGE GONCALVES, The University of Melbourne, Australia
}

Social conformity occurs when an individual changes their behaviour in line with the majority's expectations. Although social conformity has been investigated in small group settings, the effect of gender - of both the individual and the majority/minority - is not well understood in online settings. Here we systematically investigate the impact of groups' gender composition on social conformity in online settings. We use an online quiz in which participants submit their answers and confidence scores, both prior to and following the presentation of peer answers that are dynamically fabricated. Our results show an overall conformity rate of $39 \%$, and a significant effect of gender that manifests in a number of ways: gender composition of the majority, the perceived nature of the question, participant gender, visual cues of the system, and final answer correctness. We conclude with a discussion on the implications of our findings in designing online group settings, accounting for the effects of gender on conformity.

CCS Concepts: • Human-centered computing $\rightarrow$ Empirical studies in collaborative and social computing; Empirical studies in HCI.

Additional Key Words and Phrases: Social Conformity; Gender; Gender-typed Questions; Names; Avatars; Online Quiz; Majority Size; Self-reported Confidence

\section{ACM Reference Format:}

Senuri Wijenayake, Niels van Berkel, Vassilis Kostakos, and Jorge Goncalves. 2019. Measuring the Effects of Gender on Online Social Conformity. Proc. ACM Hum.-Comput. Interact. 3, CSCW, Article 145 (November 2019), 24 pages. https://doi.org/10.1145/3359247

\section{INTRODUCTION}

Conformity is a widely studied form of social influence that leads individuals to change their personal judgements and opinions when challenged by an opposing majority [2]. Deutsch and Gerard [20] explain that such behaviour is commonly motivated when individuals attempt to fit in with a group (i.e., normative influence), or are seeking guidance in uncertain situations in an attempt to be right (i.e., informational influence). As our social interactions increasingly shift towards online platforms, with over 4 billion Internet users and over $74 \%$ of those active on social media [35], investigating whether and how social conformity manifests and influences human behaviour in online group settings is important in order to facilitate positive interactions.

The literature on face-to-face social conformity suggests that individuals are influenced by a number of different determinants when conforming to the majority's opinion, such as majority size $[3,46]$ and their self-confidence $[13,65]$. Furthermore, research on the effects of gender on social influence in face-to-face groups has shown that women are typically more receptive to others' opinions while men are seen to be more influential in certain cases [22], meaning that women are

Authors' addresses: Senuri Wijenayake, wijenayakes@unimelb.edu.au, The University of Melbourne, Parkville, VIC, 3010, Australia; Niels van Berkel, n.vanberkel@ucl.ac.uk, University College London, 66-72 Gower St, London, WC1E 6EA, UK; Vassilis Kostakos, vassilis.kostakos@unimelb.edu.au, The University of Melbourne, Parkville, VIC, 3010, Australia; Jorge Goncalves, jorge.goncalves@unimelb.edu.au, The University of Melbourne, Parkville, VIC, 3010, Australia.

Permission to make digital or hard copies of all or part of this work for personal or classroom use is granted without fee provided that copies are not made or distributed for profit or commercial advantage and that copies bear this notice and the full citation on the first page. Copyrights for components of this work owned by others than the author(s) must be honored. Abstracting with credit is permitted. To copy otherwise, or republish, to post on servers or to redistribute to lists, requires prior specific permission and/or a fee. Request permissions from permissions@acm.org.

(C) 2019 Copyright held by the owner/author(s). Publication rights licensed to ACM.

2573-0142/2019/11-ART145 \$15.00

https://doi.org/10.1145/3359247

Proc. ACM Hum.-Comput. Interact., Vol. 3, No. CSCW, Article 145. Publication date: November 2019. 
generally seen to conform more than men under group pressure [24,25]. However, due to the inherent dissimilarities between physical and online groups [52], it is unclear if observations resulting from the above seminal studies apply to online settings.

More recent literature investigating the implications of gender in computer-mediated communication (CMC) settings reveal effects of partner gender on 'informational' social influence [41, 42]. These studies show that individuals are more likely to accept the opinions of a stereotypically male-charactered partner in stereotypical masculine topics and those of a stereotypically femalecharactered partner in stereotypical feminine topics. Moreover, work by Christofides et al. [15] identifies the presence of gender identification in online discussions, where men perceived interviewers with stereotypical masculine names to be more competent than interviewers with stereotypical feminine names, regardless of the topic of discussion. One shortcoming of these studies is that they are limited to exploring gender effects in pairs, and do not consider larger groups.

Furthermore, despite early expectations that reduced social presence in online settings would facilitate more unbiased modes of communication for all those involved [21,37], recent literature invalidates this notion. For example, in contexts where gender is not directly specified, individuals perceive the gender of their online correspondents through their names [15, 45], usernames [19], avatars [41, 42], and even linguistic use [32, 61], triggering stereotypical behaviour. However, the effects of these different gender cues on social conformity have not been measured in online settings.

This study aims to explore the impact of three gender-based aspects on conformity, while also validating findings from previous work on the impact of majority size and self-confidence. First, we investigate how different gender group compositions in the majority and the minority may affect online conformity behaviour of participants. Since the experiment is related to an online setting, we utilise commonly used stereotypical gendered representations (i.e., masculine and feminine avatars and names) to illustrate different gender compositions. Second, we compare these two stereotypical gendered representations (avatars and names) in terms of triggering gender-related stereotypes and gender-biased conformity. Finally, we also investigate whether the self-disclosed gender identity of participants affects their susceptibility to such gender-biased stereotypes leading to social conformity.

We deploy an online quiz containing multiple-choice questions on topics that are stereotypically seen as being of masculine (sports) and feminine (fashion) nature, as well as neutral questions. Participants first answer each question privately while providing their self-reported confidence on the selected answer. Next, our software displays a fabricated distribution of peer answers denoting a clear majority, while placing the participant in either the minority or majority. To assess the impact of gender cues on triggering stereotypical perceptions among participants, we introduce a total of three conditions: a control condition in which participants were not aware of the gender of their peers; a condition with stereotypical masculine and feminine names; and finally a condition with stereotypical masculine and feminine avatars. Subsequent to displaying the fabricated peer answers, participants are given the opportunity to change both their initial answer and self-reported confidence. We consider a change to the participant's answer to be a sign of conformity when the change is in line with the majority's opinion.

While our results did not indicate a main effect of self-disclosed gender of participants on conformity, we observe stereotypes on supposed competency based on perceived peer gender. In instances where peers are represented by gender-rich names or avatars, our results show an increase in conformity when it comes to stereotypical masculine and feminine questions. Both men and women are more likely to accept the majority's answer to a stereotypical masculine question when the majority consists of more stereotypical masculine avatars or names as compared to a majority which consists of more stereotypical feminine avatars or names. We observe a similar behaviour for stereotypical feminine questions, when the majority had more stereotypical feminine avatars or names than stereotypical masculine avatars or names. Such conformity behaviour was more frequent when peers are represented using avatars as opposed to names. Our analysis also considered the consequences of conforming to the majority in terms of final answer correctness: participants were more likely to conform to an 
incorrect answer, especially in stereotypical masculine and feminine questions. We conclude with a discussion on the implications of our findings in designing for online groups so that individuals are less influenced by stereotypical gender biases, especially with regard to 'informational' social influences.

\section{RELATED WORK}

Asch's conformity experiments $[2,3]$ are a landmark in Social Psychology research, where a significant proportion of participants (33.3\%) revised their individual judgements to agree with a clearly incorrect, yet unanimous majority, establishing the existence of conformity in group settings. A subsequent study revealed two motives behind group conformity: normative and informational influences [20]. The researchers described 'normative influence' as the tendency to conform to expectations of the majority to be 'liked' within the group, while accepting the majority's judgement to be more accurate than one's own knowledge of the situation was described as 'informational influence'. More recent work explained conformity as an outcome of individuals being driven to ensure one's belongingness to a group [17] or to fit in with the majority [44], further establishing the impact of 'normative influence' on conformity. Moreover, previous work has further emphasised the effects of 'informational influence' on conformity, where individuals turn to groups for direction in situations where the 'correct' response is unclear $[16,44]$.

As social interactions are increasingly shifting from physical paradigms to online settings, investigating whether and how social conformity manifests in online groups is vital. Next, we summarise the research that has been conducted in this area.

\subsection{Online Social Conformity}

Due to the increased use of online platforms (e.g., discussion forums, support groups, learning platforms), human interactions are increasingly taking place online $[28,58]$. Therefore, the extent to which physical social influences manifest in an online setting has been of interest to the research community.

Previous work has argued that online groups are affected by social influences similar to face-to-face groups [52], despite reduced effects in the former. This notion is further supported by literature comparing social conformity in online and offline settings. For example, work by Cinnirella and Green [18] extended Asch's 'line experiment task' by allowing participants to select their answers through computer-mediated communication (a personal computer), offering participants anonymity. The results of this experiment were compared against a traditional face-to-face group setting, and showed that conformity was still apparent in the anonymous computer-mediated group, despite demonstrating lower effects when compared to the face-to-face group. On the contrary, work by Reicher et al. [57] argues that anonymity of computer-mediated groups could lead to 'deindividuation', where individuals tend to lose self-awareness as a result of extreme involvement with the group. This in turn can encourage individuals to more strongly conform to group norms [55,56]. More recent literature investigated conformity during social watching, where people discuss social issues with others through online social networks while simultaneously watching video telecasts. The researchers observed that people tend to adopt the majority's opinion on social issues, even when they do not know the users who are posting the content [48]. Another study by Maruyama et al. [49] showed that users who actively tweet during a televised political debate changed their voting choice to reflect the majority sentiment on Twitter, further showcasing the presence of social conformity in online settings.

Furthermore, literature suggests that social conformity exhibits both positive and negative implications in online settings. Sukumaran et al. [64] explored the use of normative conformance in shaping the amount of effort users put into their contributions in online news websites. The study highlighted that when the initial comments added by other users were more 'thoughtful', subsequent participants were also motivated to contribute with similar or additional effort. The researchers concluded that conformity can be used to establish a long-term positive structure within online communities, when applicable. However, a more recent study investigating social conformity in virtual classrooms portrayed its negative influences [9]. This study required graduate students to 
answer an online quiz where a proportion of participants were shown the responses of others in the group, while the remaining participants answered the quiz independently. The resulting observations revealed high levels of conformity among students in online learning environments, rationalised by their lack of understanding of the content as well as high levels of self-doubt. More importantly, students who conformed to the majority's answers obtained fewer correct answers compared to students who answered the quiz independently.

Recently, a study based on an online support group described how mental health patients conforming to the linguistic norms of the community received better support as compared to those who did not [62]. This study set forth both positive and negative implications of conformity. On one hand, the researchers observed that conforming to the acceptable conventions of behaviour improved the sense of belonging and security within the community, so that sensitive mental health issues could be openly discussed. However, they also argued that pressure to conform to the group's norms may cause unnecessary distress to individuals seeking support from online communities.

As the literature shows that social conformity can have mixed effects in online social groups, we argue that in order to derive positive outcomes through this powerful social influence a more thorough understanding of its determinants is needed.

\subsection{Determinants of Social Conformity}

2.2.1 Majority Size and Self-confidence. Since conformity is a social influence exerted by a group's majority on the group's minority or minorities, the size of the influencing source (majority) is an obvious determinant, and has been thoroughly researched in face-to-face groups. For instance, Asch explored how conformity was affected by subsequently increasing the size of a unanimous majority [3]. He observed that against a minority of one, the influential power of the majority increased until its third member, while adding a fourth member to the majority did not generate a higher conformity influence. A subsequent study explained that larger majorities exert more pressure on individuals to conform as a result of higher normative and informational influences [33]. Moreover, a study by Lowry et al. [46] compared the conformity effects of two group sizes ( 3 and 6), when the communication was face-to-face and computer-mediated. Their results show that while conformity effects heightened in both conditions as the group size increased, the effect was minimised in the computer-mediated condition. Thus, it is plausible that online groups may not be affected by adverse influences of social conformity, even with increasing group sizes. However, this notion is yet to be systematically tested in online settings.

Previous work has shown that conformity is driven by informational influences, where individuals confide in judgements of a group's majority as the accurate interpretation of a given situation, disregarding their own judgements [20]. This notion implies that confidence in one's judgements may play a role in determining the likelihood of a person conforming. This relationship has been established in face-to-face groups, where higher rates of conformity were observed when individuals displayed low confidence on their personal answers and higher confidence on group's answers [13, 60, 65]. Furthermore, Rosander and Eriksson [59] observed that individuals in online groups who considered the experimental tasks to be difficult more frequently conformed to incorrect majorities than those who did not. However, the impact of self-confidence on conformity is yet to be explored in-depth with regard to online groups.

In this paper we validate findings from previous work on the influence of majority size and selfconfidence on conformity in online settings, while also exploring the impact of gender and gender cues.

2.2.2 Gender and Gender Cues. The effects of gender on social conformity has been a topic of interest in Social Psychology research. Early literature concerning face-to-face groups revealed that women are more easily swayed by external influence than men [22], leading to higher conformity under group pressure settings [23]. Moreover, Eagly and Wood [24] explained gender differences in conformity as a result of stereotypical gender-oriented social roles that individuals are expected 
to follow in groups. For example, while men are expected to be more 'agentic' and task-oriented, women are expected to be cooperative and selfless, when placed in group settings. This notion is further emphasised in [67], where men were wrongly perceived to be more competent than women in group discussions, leading them to actively contribute opinions, while women were more likely to agree with the group's opinions demonstrating cooperative social behaviour.

Despite the optimistic expectations for computer-mediated communication (CMC) to enable unbiased communication [21,37], literature emphasises that this is not always the case. While men and women do not differ in their basic online skills, previous work has shown that women tend to self-assess their skills as being less advanced when compared to the self-assessment of men, which can affect their confidence and online behavior [31]. In addition, Matheson [50] observed that individuals form stereotypical perceptions of their online correspondents based on gender, where women were perceived to be more cooperative and less exploitative than men. Moreover, work by Postmes and Spears [54] highlighted that 'deindividuation' caused by computer-mediated communication tend to enhance stereotypical perceptions and behaviour among individuals.

More recent work further establishes the notion that men and women respond to the gender of their peers differently, especially when connected with stereotypical masculine and feminine tasks. For example, in a study by Christofides et al. [15] where participants rated the effectiveness of their interviewers subsequent to an online discussion, women rated interviewers with stereotypical feminine names as more competent in stereotypical feminine topics with similar results for interviewers with stereotypical masculine names in stereotypical masculine topics. However, men rated interviewers with stereotypical masculine names as more competent regardless of the topic of discussion. Another study by Lee [41] demonstrates similar behaviour where women conformed to stereotypically female-charactered partners in stereotypical feminine topics and stereotypically male-charactered partners in stereotypical masculine topics, while men displayed greater conformity to stereotypically male-charactered partners even in stereotypical feminine topics. While existing literature adequately highlights the prevalence of gender stereotyping in online settings, they limit their analysis to pairs of individuals. Thus, in this paper we explore the implications of gender stereotypes on social conformity with regard to small online groups.

Moreover, existing literature reveal that users of CMC platforms infer gender of their peers through cues such as names [15, 45, 51], usernames [19] and avatars [41, 42]. These cues play a significant role in triggering gender stereotypes in online settings when gender is not explicitly provided. For example, a study by Lee [42] demonstrates that participants inferred the gender of their anonymous partners based on a randomly assigned gender-marked character (which may or may not correctly represent partner gender), where stereotypical masculine characters triggered higher conformity than stereotypical feminine characters. Moreover, while previous work has shown that certain cues may be more powerful in triggering stereotypical perceptions of others [43], their effects on conformity is yet to be explored.

\section{METHOD}

We aim to investigate the impact of group gender composition on online social conformity across different question types and gender cues. To control these aforementioned variables, while simultaneously simulating a plausible real-world online setting, we deployed our study as an online multiple-choice question (MCQ) quiz. Previous work has successfully utilised MCQ quizzes to capture occurrences of online social conformity [9, 39, 59].

\subsection{The Quiz}

The quiz contained 39 objective MCQ questions equally distributed among topics which are stereotypically perceived to be masculine (sports), feminine (fashion), and neutral (general knowledge). We include the complete list of questions used in the quiz as supplementary material. The choice of stereotypical masculine and feminine topics was based on existing literature studying gender-stereotyping 
in computer-mediated communication [40-42]. The results from these studies established that the chosen question topics (fashion and sports) successfully trigger gender stereotypes. We chose general knowledge based topics to represent neutral questions. This is in line with recent literature on conformity that deployed similar MCQ quizzes and showed no gender differences in conformity for these questions $[39,59]$.

Once the topics were decided as fashion (feminine), sports (masculine), and general knowledge (neutral), we extracted potential multiple-choice questions covering these topics from popular online question repositories such as Britannica and Sporcle for sports, BuzzFeed for fashion, and Syvum for other general knowledge questions. Next, we employed a similar approach used in previous work on social conformity to rate the perceived masculinity and femininity of questions [40-42]. Two of the paper's authors individually rated the perceived masculinity and femininity of the questions on ten-point Likert scales (1 - Not at all masculine/feminine to 10 - Extremely masculine/feminine). Perceived masculinity ratings were reverse-scored and added to the perceived femininity rating to arrive at a final score for each question. For neutral questions, the same authors rated how gender-biased the questions were in a ten-point Likert scale, regardless of the perceived masculinity or femininity of the question (1 - Not at all gender-biased to 10 - Extremely gender-biased). We then aggregated the scores given to each question and selected the top 13 questions from each topic (i.e., sports, fashion, and general knowledge) to be included in the quiz to represent stereotypical masculine and feminine questions, as well as neutral questions. The purpose of having different types of questions was to determine whether gender bias relative to conformity is heightened due to question stereotypes. We only selected objective questions from the aforementioned topics as we intend to determine the impact of conformity on final answer correctness.

The quiz follows the structure illustrated in Fig. 1. First, the user is instructed to attempt the question by themselves and select the correct answer option (see Step 1 in Fig. 1). Upon choosing an answer option, the user is prompted to rate their confidence in the chosen answer. Self-reported confidence levels were denoted using a scale ranging from $0-100$ with higher values representing higher levels of confidence. Subsequently, the user is presented with a fabricated diagram claiming to show how their peers have answered the same question (see Step 2 in Fig. 1). The fabricated peer answers were dynamically generated by our software to show the distribution of votes from other participants across two answer options in such a way that one answer secured a clear majority of votes. This notion of using fabricated feedback diagrams to investigate social conformity was inspired by work from Rosander and Eriksson [59]. Following the display of the peer answers, the user is then given the option to maintain their original answer or change the originally selected answer option and confidence (see Step 3 in Fig. 1).

\subsection{Gender Cues}

We introduce three conditions to analyse the impact of different gender cues in triggering stereotypes influencing social conformity. The conditions differ only by the peer answer diagrams presented to the participants, as shown in Step 2 of Fig. 1. We deployed a control condition (see Fig. 1 Step 2 (a)) where a vote was represented by a grey square, removing any gender cues from peer answers. In contrast, the second condition (referred to as the 'names' condition) displayed names of peers (see Fig. 1 Step 2 (b)), and the final condition (referred to as the 'avatars' condition) represented peers through two stereotypically gendered masculine and feminine silhouette avatars (see Fig. 1 Step 2 (c)).

We highlight that the choice of gendered representations (names and avatars) used in the study was based on the fact that these representations are commonly seen in many online social platforms such as Facebook, Goodreads, and Learning Management Systems such as SAP Litmos, where user decisions can be influenced by others. We do not consider a gender-neutral avatar as it is less likely to trigger gender-based stereotypes. We highlight that the study already includes a control condition (with no gender cues), against which we compare the conditions with gender cues (both names and avatars).

Furthermore, we ensured that the representation of a peer answer had an identical length in all conditions to ensure visual consistency in the presentation and avoid any additional bias. 


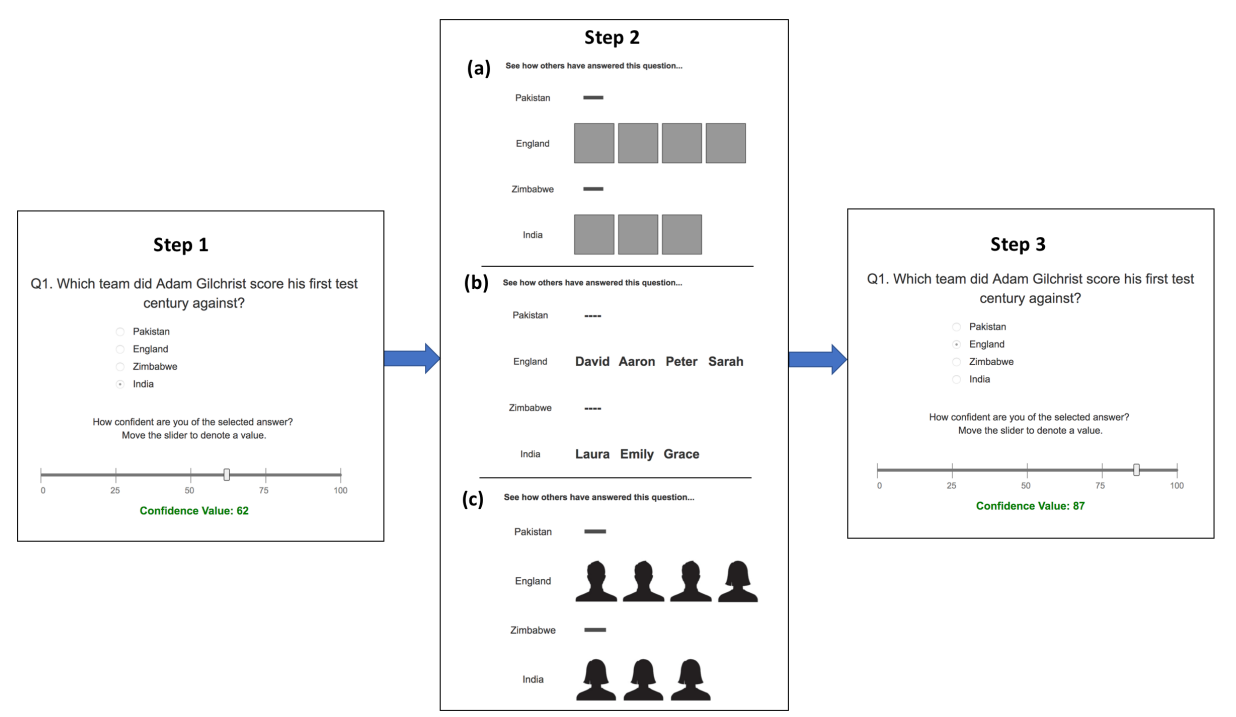

Fig. 1. Steps to be followed during the quiz : Step 1: Initial answer and confidence, Step 2: View peer answers (participants will see the representation pertaining to each condition), Step 3: Final answer and confidence.

3.2.1 Names. Our 'names' condition is based on the literature that explains how names of individuals can be used to infer gender in online settings [15, 45]. We extracted the top most frequently used stereotypical masculine and stereotypical feminine names in the chosen community, so that participants are more likely to be familiar with the displayed peer names. We selected names that clearly suggested the gender of the peer (such as 'David' and 'Sarah') and avoided using names (such as 'Sam') which can be perceived as more ambiguous.

3.2.2 Avatars. Similarly, literature supports the notion that avatars (a frequently used method of online self-representation) are strong cues in triggering gender perception in online settings - in turn eliciting gender stereotypical behaviour $[40,53]$. To minimise the effect of other possible traits that could be inferred from avatars such as participant race [4,29], ethnicity [66], personality [26] and age [68], we utilised two frequently used silhouette avatars with minimum user cues to represent men and women in the majority and the minority (as displayed in Fig. 1 (c)).

\subsection{Group Composition}

We chose to work with an overall group size of seven in all three conditions (i.e., the size of the majority plus the size of the minority - excluding the participant attempting the quiz - sums up to seven). Previous work that focused on the effects of group gender composition on influence and group work use either same or mixed-gender dyads [14] or groups of 4 participants [34], which restricted the possible gender group compositions that could be tested. In contrast, a group size of seven allowed us to test the following gender group compositions in a majority as well as a minority, with different gender group sizes.

- F > M: The group consists of more stereotypical feminine names or avatars than stereotypical masculine names or avatars.

- M > F: The group consists of more stereotypical masculine names or avatars than stereotypical feminine names or avatars.

- $\mathrm{M}=\mathrm{F}$ : The group consists of an equal number of stereotypical masculine and feminine names or avatars.

- F: The group consists of only stereotypical feminine names or avatars.

- M: The group consists of only stereotypical masculine names or avatars. 
We did not choose a group size higher than 7 as this would require us to include more questions in the quiz (already at 39 questions) - further increasing participant strain. Each question in the quiz displayed a fabricated peer answer diagram that reflected a specific group gender composition (out of $\mathrm{F}>\mathrm{M}, \mathrm{M}>\mathrm{F}, \mathrm{M}=\mathrm{F}, \mathrm{M}$ and $\mathrm{F}$ ) for the majority and the minority, while maintaining a total group size of 7 . The majority group size ranged from 4 to 6 peers while the corresponding minority ranged from 3 to 1 peers. For example, a possible group composition could be a majority of 5 with 3 stereotypical feminine avatars/names and 2 stereotypical masculine avatars/names $(\mathrm{F}>\mathrm{M})$, alongside a minority of 2 with 1 stereotypical masculine avatar/name and 1 stereotypical feminine avatar/name $(\mathrm{M}=\mathrm{F})$.

Once a participant submits their initial answer and confidence, our software dynamically produced a fabricated peer answers diagram which displayed a manipulated distribution of votes across two answer options, placing the participant either in a majority ( 9 out of 39 questions) or a minority (30 out of 39 questions). For instance, the Fig. 1 (c) illustrates a question where the participant was placed in an minority consisting of 3 stereotypical feminine avatars $(\mathrm{F})$, against a majority consisting of 3 stereotypical masculine avatars and one stereotypical feminine avatar $(\mathrm{M}>\mathrm{F})$.

We counterbalanced the question types (i.e., neutral, masculine, feminine) and group gender compositions in such a way that, for a given group gender composition, an equal number of participants answered questions pertaining to each question type.

\subsection{Participants and Procedure}

We recruited 54 participants ${ }^{1}$ from different educational backgrounds which included engineering, science, arts and design, commerce and marketing fields. The group consisted of 27 women and 27 men. We do not know whether the participants were trans or cisgender as participants did not disclose this information. Participants' age ranged between 18 - 34 years. All participants were recruited through an online notice board. Participants were equally distributed among the 'control', 'names', and 'avatars' conditions, with 9 men and 9 women in each group. Within each condition, an equal number of men and women were assigned to question sets 1,2, or 3 to counterbalance both questions types and group gender compositions.

The experiment was conducted in a laboratory with one participant per session and under the supervision of a researcher. Participants were informed that the objective of the study was to determine the importance of peer feedback in online settings, as the true purpose of the study could not be disclosed prior to the quiz as expected in studies investigating conformity behaviour [63].

Participants then completed an online form which collected their gender, age, and educational background. We highlight that participants were given the opportunity to self-disclose their gender without being restricted to binary gender identities (or not disclose at all) [36]. Upon submitting their demographic details, participants were randomly assigned to either the control, 'names', or 'avatars' condition. In the home screen of our application, the participants were greeted by a conversational agent named 'QuizBot', which assisted participants in familiarising themselves with the environment through a training question as displayed in Fig. 2.

Training was considered essential to ensure that the participants were aware of the process to be followed during the quiz. We utilised the 'QuizBot' to provide step-by-step instructions to participants during the training while minimising the intervention of researchers. This enabled us to simulate a typical online setting where participants were by themselves while minimising any gender biases that could occur due to the presence or intervention of the researchers. After training, the bot directed participants to the quiz. All participants completed the quiz individually, repeating the steps described in Fig. 1.

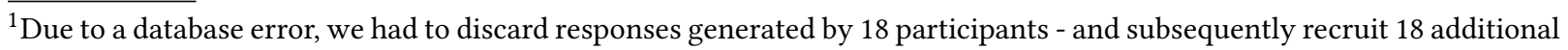
participants to compensate for this error. Therefore, while we recruited 72 participants in total, the findings of this study originate from 54 participants.
} 


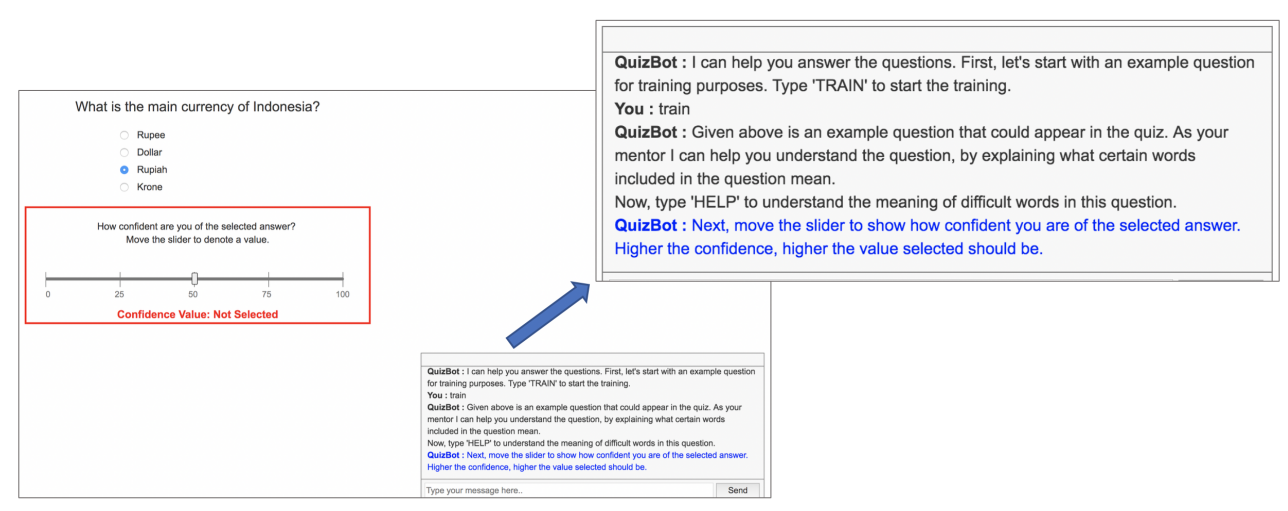

Fig. 2. QuizBot assisting the participants through step-by-step instructions during training.

Upon completion of the quiz, participants participated in a brief semi-structured interview in which they were debriefed on the true objective of the study. Subsequently, we enquired what were the main reasons behind their decisions to change their initial answer during this quiz. We were also interested in whether they sought out the answers from peers of a particular gender with regard to certain types of questions, to understand whether and why participants actively inferred gender of others through the feedback they received when deciding the final answer. Participants were also asked to comment on the strength of the gender cues (in 'names' and 'avatars' conditions), to determine the effect of these gender cues in suggesting stereotypical behaviour in online settings.

The experimental design was approved by the Ethics Committee of our university. The experiment lasted for approximately 60 minutes per participant, including briefing, training, completing the quiz, and final interview. Each participant received a $\$ 15$ gift voucher for participation.

\section{PILOT}

Before running our experiment, we conducted a pilot study with 20 participants (10 men and 10 women). In this pilot, study participants individually answered the same set of stereotypically perceived masculine, feminine, and neutral questions under lab conditions (they were not shown other people's answers). This resulted in a total of 780 responses. We observed that for all questions, participant answers dispersed among mostly two answer options. Moreover, for 20 (out of 39) questions, the majority of participants selected the correct answer. The overall group accuracy was approximately $38 \%$ (299 out of 780 responses). Overall, we identified that participants were most correct in answering neutral questions ( $45 \%$ correct responses) and least correct on the questions that are stereotypically perceived as being of masculine nature (28\% correct responses). However, we did not observe any statistically significant relationships between gender and different question types, meaning that popularly perceived stereotypical relationships between participant gender and the perceived nature of questions were not reflected in our answer set.

Next, we arranged the answer options of each question based on the descending order of the number of votes they received during the pilot study. This data was then used to determine the arrangement for the majority and minority groups when fabricating peer answers. For instance, for the question illustrated in Fig. 1, "India" and "England" were the top two answer options chosen by the pilot participants. Thus, when a study participant attempting the quiz selected "India" as their initial answer, our software dynamically fabricated the illustrated diagrams placing "England" as the majority answer. This ensured that the majority was always placed in a reasonable answer option, regardless of being correct or incorrect. 


\section{RESULTS}

We asked 40 questions from each of the 54 participants (1 training question and 39 quiz questions). Responses to training questions were removed from the dataset prior to analysis, which resulted in 2106 answered questions. Our software placed participants both in the majority (for 9 out of 39 questions) as well as in the minority (for 30 out of 39 questions) to cause no suspicion about the authenticity of the peer answers. Thus, the participants were in the majority in 486 questions and in a minority for the remaining 1620 questions (equally distributed among topics which are stereotypically perceived to be masculine, feminine, and neutral). We note that our intention was not to compare results between majority and minority groups, but rather to explore the impact of different group gender compositions on conformity behaviour among individuals. Upon seeing the answers of their peers, participants could either:

- Change both answer option and confidence level.

- Change only their answer option.

- Change only their confidence level.

- Make no change to their initial answer.

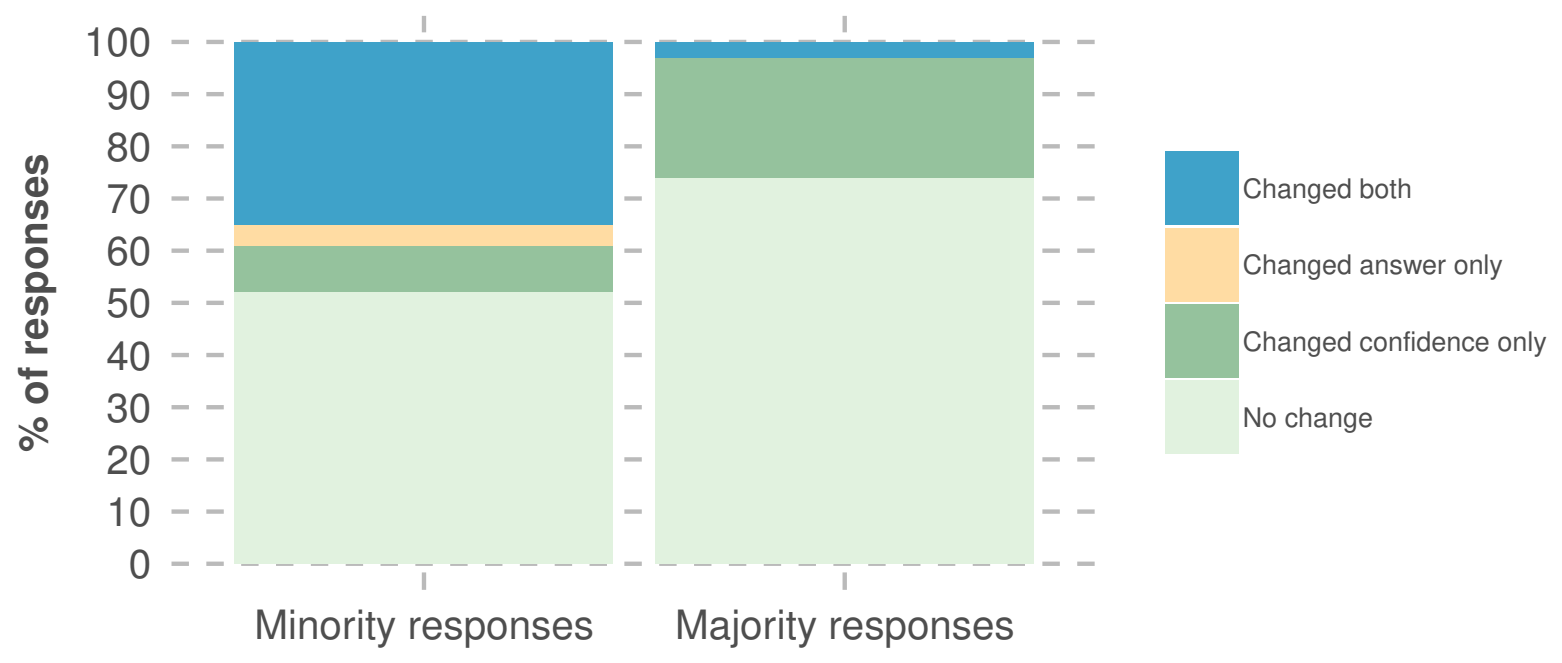

Fig. 3. Distribution of changed responses across the four post-feedback response types.

Our results show that all participants changed their initial response (answer option and/or confidence) at least once during the quiz, resulting in a total of 904 changes with an average of 16.74 changes ( $\mathrm{SD}=7.33$ ) per participant. Out of these 904 changes, 777 were made by participants placed in the minority and the remaining 127 from participants placed in the majority. The distribution of the final responses of participants across the aforementioned response types is shown in Fig. 3.

As illustrated by Fig. 3, the act of conformity (changing one's answer) occurred predominantly when participants were placed in the minority (approximately $39 \%$ of the minority responses). Moreover, participants were more likely to increase their confidence (approximately $23 \%$ ) than changing their answer when placed in the majority. Only $3 \%$ of the responses generated by participants placed in majorities demonstrated a change in their answers to that of the minority.

\subsection{Model Construction}

The objective of this study was to determine the impact of participant gender and group gender composition across different question types on online social conformity. Thus, we considered the impact of the following 15 predictor variables on whether an individual will conform to the majority's answer. 
$\mathbf{M}=$ num. of stereotypical masculine names or avatars, $\& \mathbf{F}=$ num. of stereotypical feminine names or avatars.

- Majority size: Size of the majority (ranging from 4 - 6).

- Minority size: Size of the minority (ranging from 3 - 1).

- Group difference: Difference between the majority group size and the minority group size (possible values : 1,3 or 5 ).

- $M$ in the majority: Number of stereotypical masculine names or avatars in the majority group (ranging from 0 - 6).

- F in the majority: Number of stereotypical feminine names or avatars in the majority group (ranging from 0 - 6).

- M in the minority: Number of stereotypical masculine names or avatars in the minority group (ranging from $0-3$ ).

- F in the minority: Number of stereotypical feminine names or avatars in the minority group (ranging from $0-3$ ).

- $\Delta(\mathbf{M}-\mathbf{F})$ in the majority: The difference between the number of stereotypical masculine names or avatars in the majority and the number of stereotypical feminine names or avatars in the majority (ranging from -6 to +6 , positive values when there are more masculine names or avatars).

- $\Delta(\mathbf{M}-\mathbf{F})$ in the minority: The difference between the number of stereotypical masculine names or avatars in the minority and the number of stereotypical feminine names or avatars in the minority (ranging from -3 to +3 , positive values when there are more masculine names or avatars).

- $\Delta$ (M in the majority - $\mathbf{M}$ in the minority): The difference between the number of stereotypical masculine names or avatars in the majority and the number of stereotypical masculine names or avatars in the minority (ranging from -3 to +6 , positive values when there are more masculine avatars or names in the majority than in the minority).

- $\Delta$ (F in the majority - $F$ in the minority): The difference between the number of stereotypical feminine names or avatars in the majority and the number of stereotypical feminine names or avatars in the minority (ranging from -3 to +6 , positive values when there were more feminine avatars or names in the majority than in the minority).

- Question type: Masculine, feminine, or neutral question type.

- Initial confidence: Participant's confidence in their answer prior to revealing the distribution of peer answers (ranging from 0 to 100, higher values for higher confidence).

- Gender: Participant's self-disclosed gender.

- User ID: An unique identifier assigned to a given user during the quiz.

We used the R package lme4 [8] to perform a generalised linear mixed-effects model (GLMM) analysis of the relationship between the aforementioned predictors and participant conformity. A GLMM allows us to identify the effect of a set of predictors on an outcome variable (conformity) while following an arbitrary (i.e., possibly non-normal) distribution.

We utilised three separate models to analyse data under the three experimental conditions 'names', 'avatars', and control condition. As the control condition did not display gender cues, the corresponding model did not consider gender-oriented predictors. Moreover, we considered a change in the initial answer option (with or without a change in initial confidence level) to that of the majority, as an indication of conformity behaviour. We observed that in some situations participants also reduced their confidence on the selected answer without conforming to the majority's answer option. However, we did not consider a reduction in confidence when challenged by a majority as conformity behaviour. We specified participant (User ID) as a random effect to account for individual differences in our model.

Following model selection (incremental addition of variables based on their predictive power), we arrived at three models for each condition, demonstrating statistically significant main effects and interactions as illustrated in Table 1 . The $R^{2}$ values given in Table 1 represents the percentage of the variance in accuracy explained by each model. Moreover, for each model we performed a likelihood 


\begin{tabular}{|c|c|c|c|c|c|c|}
\hline \multirow[t]{2}{*}{ Predictor } & \multicolumn{2}{|c|}{ Control } & \multicolumn{2}{|c|}{ Names } & \multicolumn{2}{|c|}{ Avatars } \\
\hline & Coef. & P-value & Coef. & P-value & Coef. & P-value \\
\hline Group difference & 0.949 & $<2 e-16$ & 0.727 & $<2 \mathrm{e}-16$ & 0.711 & $<2 e-16$ \\
\hline Initial confidence & -0.021 & $9.57 \mathrm{e}-05$ & -0.023 & $6.09 e-08$ & -0.023 & $5.28 \mathrm{e}-08$ \\
\hline Question type (fem) & -0.888 & 0.036 & 0.544 & 0.051 & - & - \\
\hline Question type (mas) & 0.591 & 0.145 & 0.685 & 0.015 & - & - \\
\hline Question type (mas) : Gender (m) & -1.964 & 0.022 & - & - & - & - \\
\hline Question type (fem) : Gender (m) & -0.569 & 0.509 & - & - & - & - \\
\hline Question type (neu) : Gender (m) & -0.939 & 0.271 & - & - & - & - \\
\hline $\begin{array}{l}\text { Question type (mas) : Gender }(\mathrm{m}) \\
: \Delta \mathrm{M}-\mathrm{F} \text { in maj. }\end{array}$ & - & - & 0.088 & 0.245 & 0.244 & 0.003 \\
\hline $\begin{array}{l}\text { Question type (fem) : Gender }(\mathrm{m}) \\
: \Delta M-F \text { in maj. }\end{array}$ & - & - & -0.166 & 0.037 & -0.151 & 0.056 \\
\hline $\begin{array}{l}\text { Question type (mas) : Gender (w) } \\
: \Delta M-F \text { in maj. }\end{array}$ & - & - & 0.209 & 0.008 & 0.416 & $4.48 e-06$ \\
\hline $\begin{array}{l}\text { Question type (fem) : Gender (w) } \\
: \Delta M-F \text { in maj. }\end{array}$ & - & - & -0.058 & 0.443 & -0.223 & 0.006 \\
\hline$R^{2}$ & & 349 & & 392 & & 371 \\
\hline$C h i^{2}$ & 209.71 & $p<0.001)$ & 196.95 & $\mathrm{p}<0.001)$ & 201.76 & $\mathrm{p}<0.001)$ \\
\hline
\end{tabular}

Table 1. Effect of predictors on participant conformity across the three models.

ratio test with the relevant null model [10] and found that all three models were statistically significant (see $C h i^{2}$ values in Table 1 ). To ensure the validity of the model, we checked for the existence of multicollinearity. All predictors across the three models had variance inflation factors well below the often-used threshold of 5 to detect multicollinearity [30].

Following model construction, we present a more detailed look at the significant features. We only considered the responses which placed participants in a minority, as the dependent variable was determining conformity behaviour.

\subsection{Group Size and Initial Confidence}

Our results show that in all three models group difference (difference between the majority size and the minority size) and initial self-reported confidence of participants demonstrated statistically significant main effects on conformity behaviour. This suggests that as the size difference between the majority and the minority groups increased, participants in all three conditions were more likely to conform. The likelihood of participants conforming to the majority in all three conditions is illustrated by Fig. 4. For each participant, the 'likelihood' of conforming for a given group difference was calculated as the proportion of conformity responses out of total responses where the participant faced the relevant group difference. For example, given that each participant was placed 12 times in minorities with a group difference of 1 , if a participant ' $A$ ' conformed in 3 such responses, the 'likelihood' of 'A' conforming to a group difference of 1 was considered as $25 \%$. Moreover, we observed 


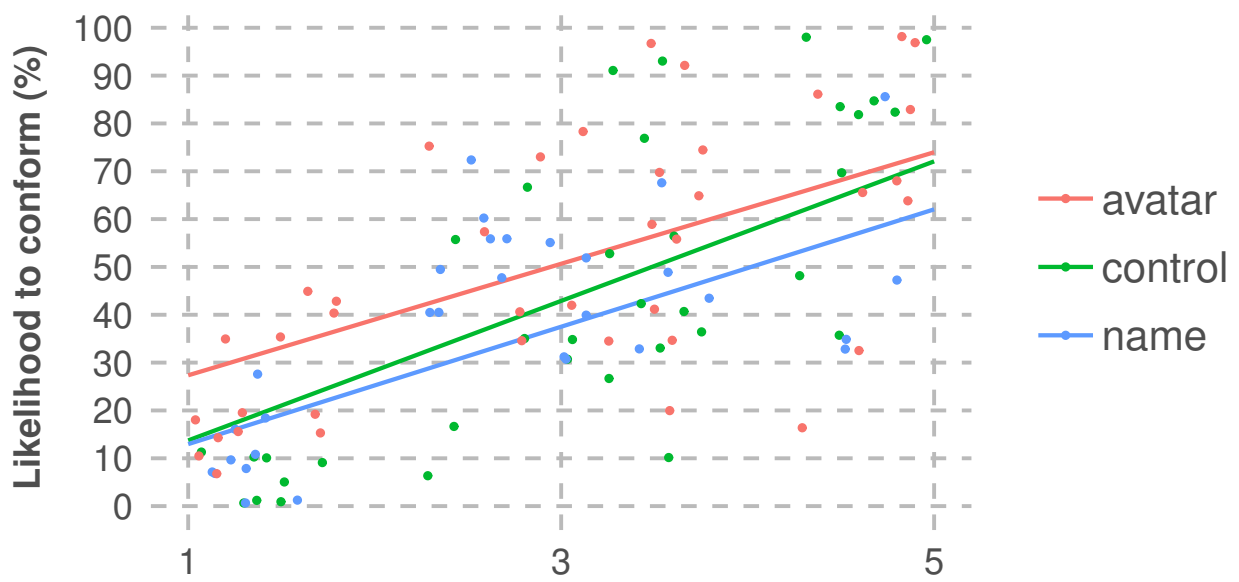

Difference between majority and minority group size

Fig. 4. The likelihood of participants conforming to the majority in the control, 'names' and 'avatars' conditions.
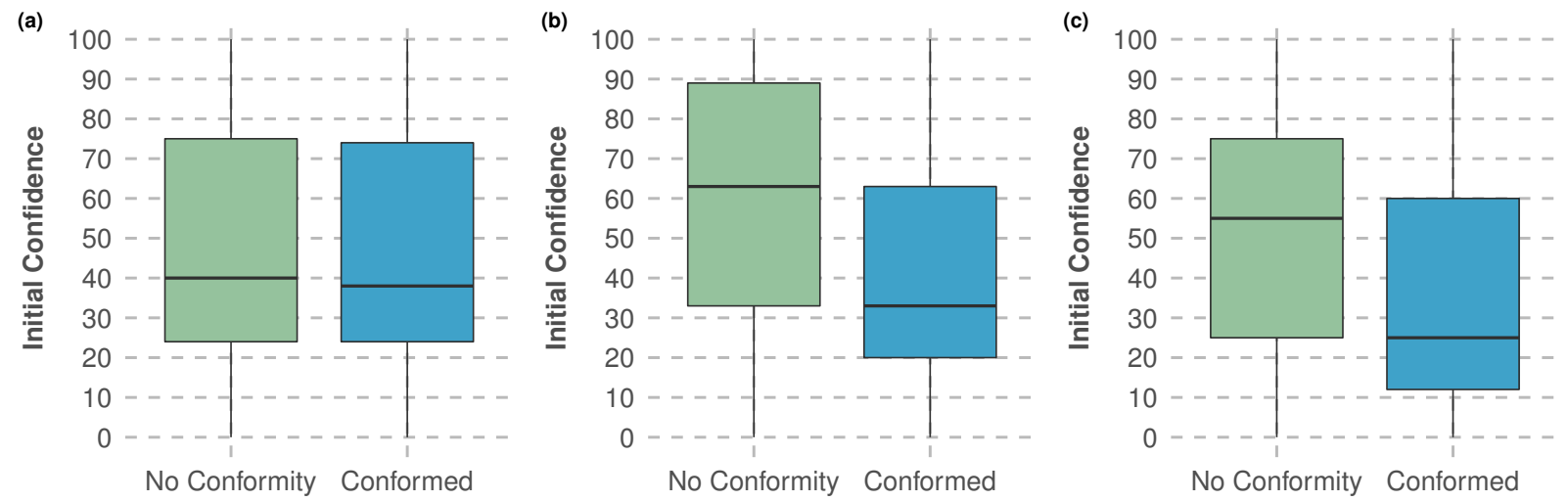

Fig. 5. Initial confidence of participants and conformity behaviour across the three conditions: (a) control, (b) names, and (c) avatars.

that the effect of group difference was strongest in the control condition (See Fig. 4), whereas in the conditions with gender cues this effect was considerably weaker.

Moreover, as the self-reported initial confidence on answers increased, participants were less likely to conform to the majority. The effect of initial confidence on conformity was strongest in the 'avatars' condition and weakest in the 'control' condition. This is illustrated in detail in Fig. 5. The confidence levels of participants in all three conditions ranged between $0-100$ in conformity responses as well as in non-conforming responses. However, we observed differences in the median values of conforming and non-conforming responses across all three conditions. In the control condition, participants who conformed to the majority displayed a median of 38, where as those who did not conform to the majority demonstrated a median value of 40 (See Fig. 5 (a)). Similarly, we observed the median values 33 and 63 for participants of the 'names' condition who conformed and did not respectively (See Fig. 5 (b)). This trend continued in the 'avatars' condition (compared to the control), where participants who conformed to the majority displayed a median initial confidence of 25 and participants who did not conform displayed a median value of 55 (See Fig. 5 (c)). In general, our results consistently show that individuals who displayed higher confidence on their initial answers were less likely to be impacted by the majority. 


\subsection{Gender and Gender Cues}

In the control model we observed that participants were less likely to conform on questions stereotypically perceived as being of feminine nature when compared to neutral questions. Men and women showed no statistically significant differences in conformity behaviour. However, we observed that men were less likely to conform to questions stereotypically perceived as being of masculine nature when compared to women (See Fig. 6). No similar interactions were observed between participant gender and the different question types.

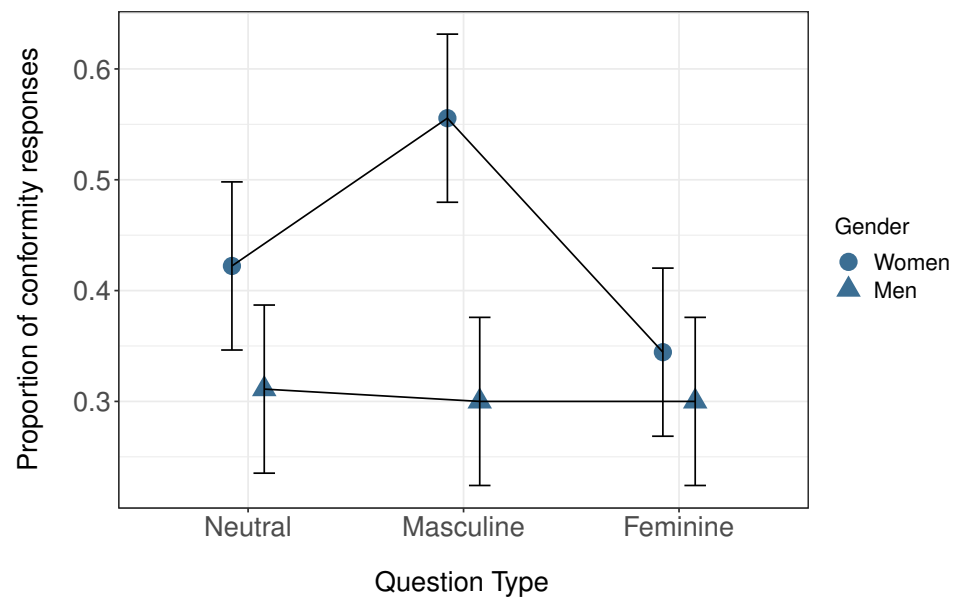

Fig. 6. Interaction between question type and participant gender in the control condition.

The 'names' model introduced gender cues to the peer answers by displaying the supposed first names of the peers to the participants. In contrast to the control model, the 'names' model suggest that participants were more likely to conform to questions stereotypically perceived as being of masculine nature than neutral questions.

As gender cues pertaining to peer answers were present in the 'names' condition, we focused on analysing any possible effects from the gender composition (operationalised by the stereotypical masculine or feminine peer names) of the majority and the minority groups on conformity behaviour. Even though we did not observe any main effects from group gender compositions, there were statistically significant interactions between participant gender, question type, and the difference between the number of stereotypical masculine and feminine names $(\Delta M-F)$ in the majority. This effect is illustrated in Fig. 7, which shows the density of conformity responses for the three question types as the difference between the number of stereotypical masculine and feminine names in the majority goes from -6 (a majority with 6 stereotypical feminine names) to +6 (a majority with 6 stereotypical masculine names). The dashed lines represent the average difference between the number of stereotypical masculine and feminine names in the majority for each question type. Negative values across the scale represent majorities with more stereotypical feminine names, while positive values represent majorities with more stereotypical masculine names.

Based on the results of our model we observed that women were more likely to conform to questions stereotypically perceived as being of masculine nature when the majority had more stereotypical masculine names than stereotypical feminine names. This is illustrated in Fig. 7 (a) by the higher density of masculine questions among the positive differences in the majority. Similarly, men found a majority with more stereotypical feminine names than stereotypical masculine names more agreeable when answering the feminine questions. Other interactions between majority gender composition, question type and participant gender were not statistically significant.

Our third model was based on the responses generated by participants in the 'avatars' condition. As in the 'names' model, we did not observe any main effects from group gender composition in the 

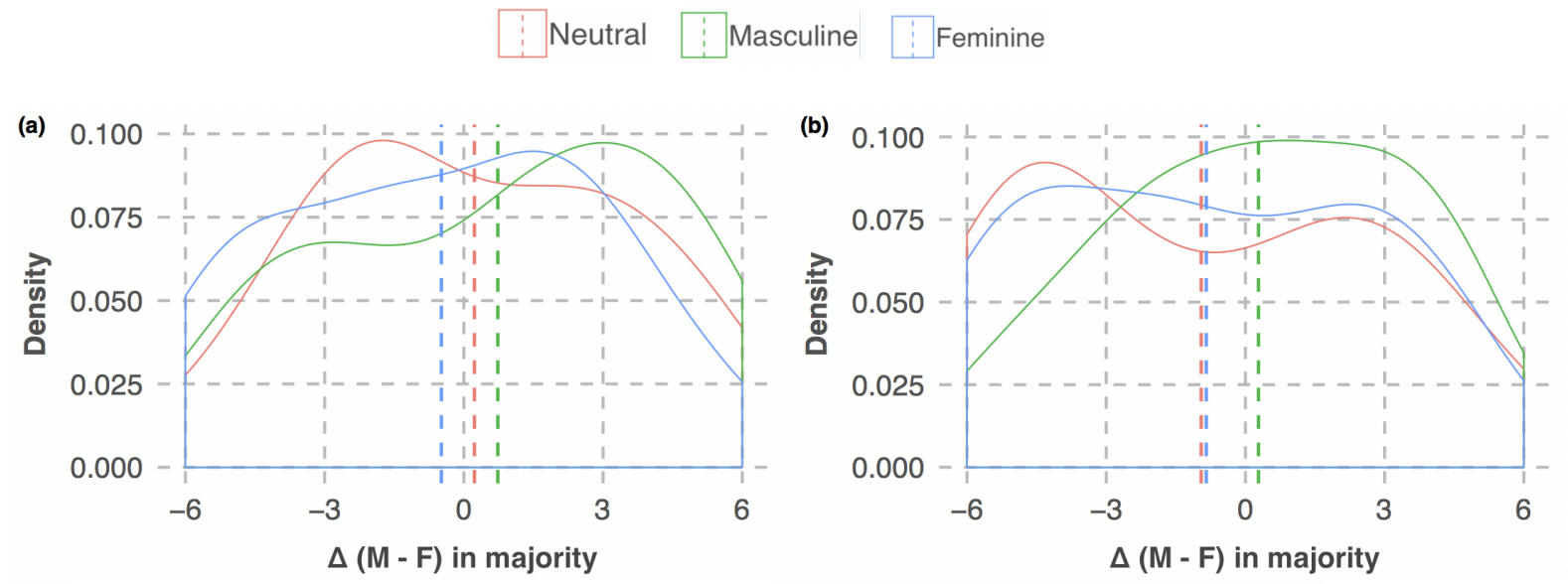

Fig. 7. Interaction between question type and difference between the number of stereotypical masculine and feminine names in the majority among (a) women and (b) men.

'avatars' model. However, our results show statistically significant interactions among the difference between the number of stereotypical masculine and feminine avatars $(\Delta \mathrm{M}-\mathrm{F})$ in the majority, question type and participant gender. The effect of the aforementioned variables on conformity is illustrated in Fig. 8, and shows a similar distribution as Fig. 7.
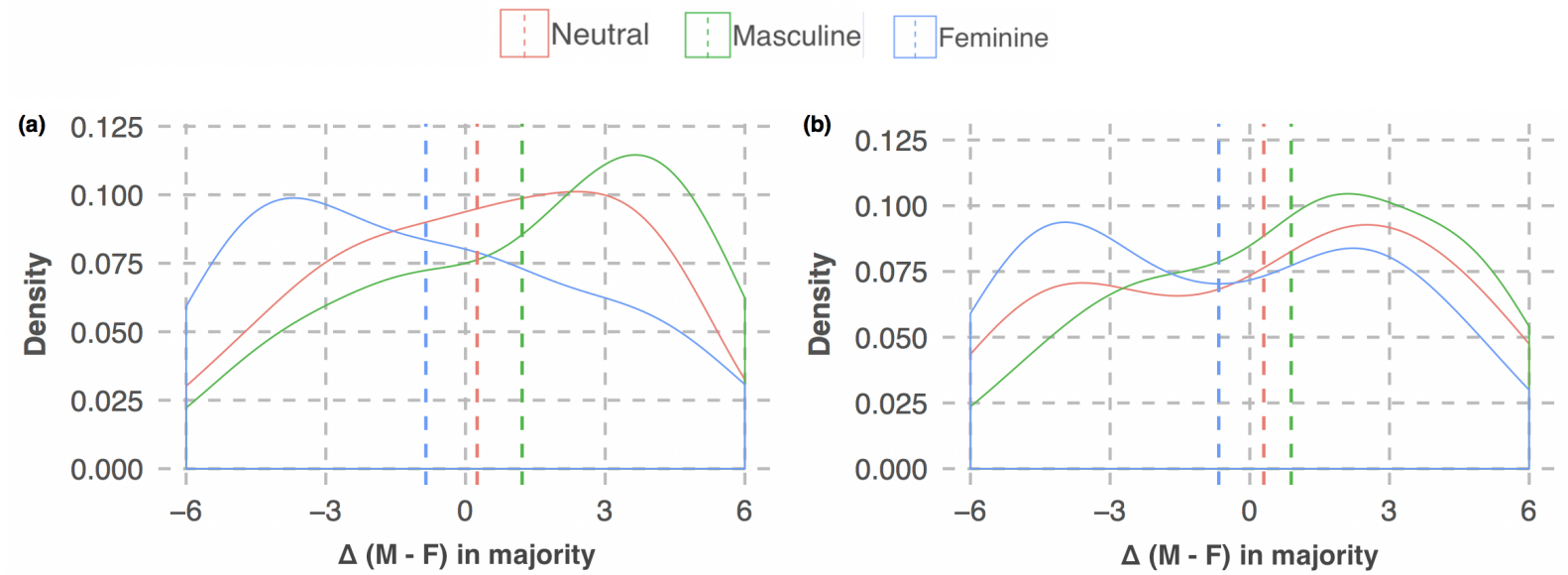

Fig. 8. Interaction between question type and difference between the number of stereotypical masculine and feminine avatars in the majority among (a) women and (b) men.

\subsection{Conformity and Answer Correctness}

Beyond identifying the impact of the previously mentioned determinants on conformity, we also quantify the effect of social conformity on the correctness of answers. All MCQ questions included in the quiz were objective questions with one correct answer. Moreover, since the distribution of peer answers was decided based on the participant's initial answer and aggregated results of the pilot study, we emphasise that the majority may have been positioned in correct answers as well as in incorrect answers. Thus, conforming to the majority may result in the a correct final answer or an incorrect final answer. Table 2 displays the distribution of correct and incorrect conformity responses across the three conditions. 


\begin{tabular}{cccccc}
\hline \multicolumn{5}{c}{ Conformity Responses $=\mathbf{6 3 1}$} \\
\hline \multicolumn{2}{c}{ Control $=\mathbf{2 1 1}$} & \multicolumn{2}{c}{ Names $=\mathbf{1 8 1}$} & \multicolumn{2}{c}{ Avatars $=\mathbf{2 4 9}$} \\
\hline Correct & Incorrect & Correct & Incorrect & Correct & Incorrect \\
95 & $\mathbf{1 0 6}(\sim \mathbf{5 0 \%})$ & 78 & $\mathbf{1 0 3}(\sim \mathbf{5 7 \%})$ & 115 & $\mathbf{1 3 4}(\sim \mathbf{5 4 \%})$ \\
$\mathrm{N}=34$ & $\mathrm{~N}=32$ & $\mathrm{~N}=24$ & $\mathrm{~N}=20$ & $\mathrm{~N}=42$ & $\mathrm{~N}=30$ \\
$\mathrm{M}=33$ & $\mathrm{M}=44$ & $\mathrm{M}=26$ & $\mathrm{M}=47$ & $\mathrm{M}=44$ & $\mathrm{M}=52$ \\
$\mathrm{~F}=28$ & $\mathrm{~F}=30$ & $\mathrm{~F}=28$ & $\mathrm{~F}=37$ & $\mathrm{~F}=29$ & $\mathrm{~F}=54$ \\
\hline
\end{tabular}

Table 2. The distribution of correct and incorrect conformity responses across the three conditions ( $\mathrm{N}=$ Neutral questions, $\mathrm{M}$ = Masculine questions, $\mathrm{F}=$ Feminine questions).

Table 2 shows that conforming to the majority was more likely to result in an incorrect answer ( $>50 \%$ incorrect answers) across all three conditions. Our results also show that in the conditions which included gender cues in peer answers, there were more incorrect answers in gender-typed questions than in neutral questions. We then conducted ANOVAs for each condition, to compare group differences between the three question types. While we did not observe any significant difference among the question types in the control condition, for the 'names' and 'avatars' conditions there was a statistically significant difference in incorrect responses pertaining to the question types. We then conducted a Tukey post-hoc test that showed a significant difference between group means of neutral and questions stereotypically perceived as being of feminine nature as well as neutral and questions stereotypically perceived as being of masculine nature, in both 'names' and 'avatars' conditions (with adj. $\mathrm{p}<0.05$ in both conditions).

\subsection{Qualitative Analysis}

The following qualitative analysis is based on our interview results obtained after participants completed the quiz, and aims to better understand the thought processes behind conforming or non-conforming behaviour of the participants in different situations. Three of the paper's authors were responsible for transcribing the audio recordings pertaining to the post-task interviews with the participants. We then individually applied a deductive thematic analysis [11] to the generated transcripts based on the paper's research objectives (i.e., effects of determinants such as majority size and self-confidence, stereotypical thinking, and gender cues on conformity behaviour in online settings). Following this, the three authors met to discuss and identify the main themes. Next, we present the main findings of this analysis.

5.5.1 Majority Size and Confidence. We set out to confirm the current literature on social conformity regarding majority size and confidence. Our qualitative results point at these two factors repeatedly as to why our participants chose to (not) conform to the presented questions.

First, the size of the majority was also considered as a factor by many participants. When faced with a large (opposing) majority, participants were more likely to change their answer; "When you see a significant majority, you start second-guessing. If it was something I knew $100 \%$ I would not change it. But if it was something I was very confident, but was not $100 \%$ sure, it made me second guess. When there were lots of people on the opposing majority, it made me feel that if that many selected the answer, it could be right." (P43). Smaller differences in the respective majority and minority were generally seen as less decisive; "If I have two options that I am not sure about, and the feedback is usually parallel with those two, Itend to change to the majority, unless there is a difference of one." (P60).

Second, the participant's confidence in their answer. Naturally, high confidence led to less conforming behaviour, and low confidence resulted in more frequent answer switching. As summarised by one of our participants; "Sometimes I got reassurance from the other answers. When I had no knowledge on the topic, I would get ideas from others. Meaning, I picked the answer that majority picked. It made me 
feel more comfortable." (P13). Similarly, participants point to specific questions to which they were certain of their answer (e.g., due to personal experiences such as hobbies or cultural background) and were therefore steadfast in their answer; "When I had the knowledge, I did not change my answer (e.g. currency of Indonesia)." (P03). These qualitative results align with our quantitative results as well as the existing literature.

5.5.2 Gender Stereotypes. Our qualitative findings support our choice of question topics for stereotypical masculine and feminine questions, as well as neutral questions. We observe that in the considered community, participants identify sports-related questions as masculine and fashionrelated questions as feminine expertise areas; "I think generally in our society women would care more about fashion and the knowledge that comes with that. Men are more interested in a lot of different sports. With people I know, men are more interested in sports and women in fashion. I thought it could be true for more people." (P36). Furthermore, our participants specifically state that the gender composition of their peers affects their decision to change their answer in gender-typed questions; "If it was a sports-related question, I will feel most comfortable not to change my answer in a group with women. And vice versa. But if the question was about geography orflags, it won't make any difference to me" (P40). For several of our participants, the effect of gender stereotypes was stronger than that of group size. As expressed explicitly; "If it was fashion I would look at the answer which most women have picked. I would still pick the group with more women even if they are not in the majority. Same with men for sports." (P27).

Some of our participants admit that their motivation is based on traditional stereotypes, but believe that these stereotypes can be used to their advantage; "When it was a fashion related question, I chose the answer which had more women in it. [...] It is a pretty stereotypical decision, but I had a feeling that women would know more about fashion." (P55). Finally, even when participants believe that the question gender type did not affect their judgement, they still alluded to the stereotypical nature of masculinefeminine expertise on sports and fashion. "I did not take the fashion/sports distribution and the gender as a relevant consideration. When we talk about fashion, it is not necessary that women or men know about it more. I know many men who don't like watching particular sports and some women do." (P25). The aforementioned comments were reported more or less equally among male and female participants.

5.5.3 Gender Cues. Finally, we discussed with participants assigned to the 'avatars' and 'names' conditions how they perceived their respective gender cue (i.e. either names or avatars) and revealed the gender cue presented to the other (non-control) condition. Participants reported that they would be more easily able to identify gender differences using a visual representation; "I would have easily noticed the difference between female and male groups with avatars. Maybe because we are familiar with the female and male avatar representations but not with the [use of] names." (P05). The interview data reveals two primary motivations behind the participants' preference for avatars over names. First, participants stated that avatars are less time-consuming and more 'obvious' to identify gender based on a visual representation; "I think avatars would be more obvious as the icons were defined as masculine and feminine. I personally struggled with the fashion questions, but it did not occur to me to look at females for fashion questions." (P24).

Second, a number of participants raised the fact that they might encounter names with which they are unfamiliar. Classifying names from different cultures as belonging to either a man or a woman can therefore be difficult. "With different countries it become more difficult to identify the gender from the names. In general silhouettes are more universal." (P42).

Finally, we note that although a vast majority of participants prefers the use of avatars over names, two participants state that names often provide further cues; "Names can have additional information as well. If the fashion designer was from Spain, I would choose the answers given by Spanish-like names." (P64). This is an interesting point, and reveals the many factors that can influence conformity. 


\section{DISCUSSION}

\subsection{Online Social Conformity}

Understanding social conformity and its implications on human behaviour in online group settings is imperative to facilitate positive and unbiased interactions. Existing literature related to online social conformity reveals that conformity can have both positive and negative implications. Social conformity is seen to enable communities to establish and strengthen group norms, leading to positive contributions [64] and creating a sense of belongingness and security by encouraging acceptable conventions of behaviour [62]. On the contrary, previous work has shown that students that conform to the majority in online quizzes make more errors than students who attempted the quiz independently [9]. Moreover, Sharma and De Choudhury [62] argued that pressure to conform to group norms may cause unnecessary distress to individuals seeking support from online communities. Thus, we argue that in order to benefit from the positive outcomes of conformity while minimising its adverse effects, a thorough understanding of its determinants is needed.

This study aims to extend the existing literature by exploring gender-stereotypes related to social conformity in online settings. While existing literature focuses on the effects of participant and partner gender in online settings [41, 42], we investigated these effects in larger groups - a common occurrence in online social systems. We explored the impact of group gender distribution and participant gender on the likelihood of an individual conforming to the majority when answering stereotypical masculine and feminine questions, as well as neutral questions. We also looked at the possible effects of different gender cues in triggering stereotypical behaviour in online settings.

Our results show that despite limited social presence in online settings, individuals are sensitive to available gender cues. Moreover, such gender cues were seen to trigger stereotypical perceptions on the competency of others which ultimately led to higher conformity, especially in questions which were perceived to be stereotypically masculine or feminine. While the gender of the participant itself did not have significant main effects on conformity, we observe statistically significant interactions between group gender distribution, participant gender, and stereotypically perceived question type.

Furthermore, our results show that the likelihood of an individual conforming to the majority decreases the more confident the person is, and increases as the difference between the majority size and the minority size (group difference) increases. This is in line with literature regarding face-to-face and online social conformity $[3,13,33,46,59,60,65]$. Furthermore, we note that the effect of group difference on conformity was largest in the control and lowest in the 'avatars' condition. This implies that while group difference had significant effects on conformity its influence on conformity was higher in the absence of gender cues.

\subsection{Impact of Stereotypes on Online Social Conformity}

Our quantitative and qualitative results reveal that both men and women typically conformed to a majority with more masculine avatars/names in stereotypical masculine questions, and to a majority with more feminine avatars/names in stereotypical feminine questions. Similar findings were found in previous work exploring effects of partner gender on conformity [41, 42]. These results suggest that in the presence of gender cues, conformity behaviour was influenced by gender-stereotypes in addition to the usual 'informational influences'. Even though our pilot study (where men and women were seen to be equally competent in all questions) clearly rejected such stereotypes, it is noteworthy that these stereotypes manifested so strongly in our experiment.

We further note that the effect of stereotypical masculine/feminine avatars was stronger than the effect of stereotypical masculine/feminine names in triggering gender-stereotypical thinking. Participants identified avatars to be more straight-forward, making the gender distribution of answers easier to interpret. These observations confirm existing literature on how individuals derive perceptions of the gender of their online peers [15, 41, 42, 45], and how some cues may have more influence in triggering stereotypes than others [43]. 
The use of objective questions enabled us to quantify the effects of conformity driven by genderstereotypes on answer correctness. Our results show that conformity resulted in more incorrect answers across the three conditions, despite the majority being placed in both correct and incorrect answers throughout the quiz. The introduction of gender cues resulted in more incorrect answers for stereotypical masculine and feminine questions, as compared to the control condition. Thus, we show that gender cues encourage individuals to conform to incorrect answers more frequently, especially in topics that can trigger a biased response. Since in online settings participants are naturally susceptible to higher 'informational influences' (e.g., groups of students answering an online quiz as given in [9]), it is crucial to consider what user information should be available to others.

Moreover, we emphasise that gender is only one dimension of a person's identity and that other aspects such as culture, race, age, and ethnicity can also be relevant in a given situation [12]. This study was designed to investigate the effects of gender stereotypes on conformity, as gender has been shown to trigger stereotypical responses in offline settings [22-24]. We deliberately avoided other aspects that could potentially lead to stereotypes as it would overly complicate the study and lead to confounding effects. We chose stereotypical gendered silhouette avatars and names, with minimum user cues (i.e., perceived gender of user) in an attempt to minimise the potential impact of other user traits such as race, ethnicity, and age which could potentially be inferred from richer cues. Similarly, the quiz questions were also chosen to specifically trigger gender stereotypes.

Thus, this work was intended to lay the necessary ground-work for future work exploring possible implications of other commonly observed stereotype-inducing factors on online social conformity. For example, work by Kumar [38] shows that in face-to-face groups participants are more likely to conform to older confederates than to younger confederates in estimation tasks. Moreover, although we selected a set of culturally-similar names, participants highlighted that first names of peers could potentially introduce cultural biases. For example, if the question was related to a particular country or region, peer names perceived to originate from this area could be perceived as more appealing than others.

\subsection{Implications for Design}

The findings of this study establish that, despite a reduced social presence in online settings, individuals stereotypically perceive others' competency based on available gender cues. Moreover, such gender stereotypical perceptions were seen to influence the conformity behaviour of users. While conformity may be seen as a positive outcome in settings where adopting group norms are encouraged [62], it is not desired when user decisions are unnecessarily influenced by gender stereotypes, as shown in our answer correctness analysis. Thus, we present the following design recommendations with regard to gender cues when designing online group settings, where user decisions may be negatively influenced by others.

6.3.1 Presence of Gender Cues. Recent literature has questioned whether gender cues (such as names and avatars) should be visible to others, and if so, when and how should they be visible [36]. Our findings indicate that basic cues (such as names) are sufficient to elicit gender-stereotyping and conformity, with a richer cue (avatar) heightening this behaviour. Furthermore, almost every online social platform allows users to upload their own photographs to be used as profile pictures in addition to the default avatars that we considered in this study. Previous work has established that photographs of users can act as strong gender cues [6,7], meaning that it is probable that the gender-stereotypical conformity observed in our work would be heightened. Thus, a designer should carefully consider whether collecting and displaying gender cues is relevant and value-adding from the perspective of users as well as the platform. If unnecessary cues are visible, users may miss other important information and act based on stereotypical perceptions.

While our results recommend against using obvious gender cues (such as binary-gendered avatars) in online group settings to reduce gender-stereotypical perceptions and conformity, we acknowledge that removing all indications of gender may not be easily achievable in an online group environment. 
Literature suggests that assumptions of peer gender in online settings may be derived based on perceived gender difference in linguistic use $[32,61]$ and gender disparities resulted by platform infrastructure [27], even when user information is not explicitly available. Thus, in addition to the cues discussed in this paper, designers should be mindful about the impact of other possible cues that may elicit similar gender stereotypical behaviour.

However, it is also important to acknowledge that in certain online settings user cues can be important to create trust among users and a sense of belonging within the community [47]. As such, many websites assign default user avatars upon registration, which can be problematic if these avatars contain gender cues. As shown in our study, users can stereotypically infer the competency of their peers based on gender cues (avatars and names), and display gender-biased conformity behaviour. For example, Facebook's use of user names and default binary-gendered avatars is likely to elicit gender-biased conformity in how users discuss stereotypically perceived masculine/feminine topics on the platform. A similar effect may occur in Goodreads, a platform which also uses names and binary-gendered avatars by default, and has discussion groups to allow users to review and discuss books (which can be pertaining to topics stereotypically perceived as masculine or feminine) with others in the community.

Moreover, while several platforms adopt gender-neutral default avatars, literature suggests that people frequently tend to perceive such avatars as masculine [5]. Supposed gender-neutral avatars are currently used in SAP Litmos, TalentLMS and Docebo (the top three most used Learning Management Systems in the world as of June 2019 [1]) as shown in Fig. 9, which could suggest 'men' as the default user group of the platform and thereby disregarding other gender identities. In addition, some users of these platforms may decide to upload their photographs, further increasing the likelihood of stereotype-based conformity. Given that these platforms are used to facilitate learning, our findings have important and far-reaching implications.

(a)

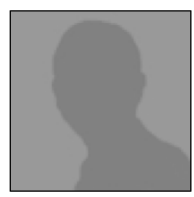

(b)

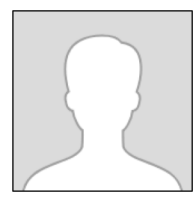

(c)

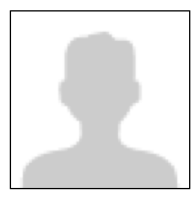

Fig. 9. Default avatars used in (a) SAP Litmos, (b) TalentLMS and (c) Docebo.

Critically, our findings establish that user representations without gender cues (the study's control condition) reduced the likelihood of gender-biased conformity. Based on these results, if avatars (or other cues) are to be used in platforms where gender-biased conformity can be detrimental, we recommend using default alternatives clearly devoid of gender cues, such as initials representing user name (e.g. Google), identicons (e.g. Github) or site specific avatars (e.g. Slack), minimising the possibility of triggering gender stereotypical perceptions and behaviour.

\subsection{Limitations}

We note the following limitations in our study. Our sample only included participants who selfdisclosed their gender-identity as either men or women, and none of the participants openly identified as trans or cisgender. It is worth noting that this study is premised on a traditional gender binary model, in which gendered senses of self fall into two clearly discernible categories. In practice, gender is far more complex than this: what it means to be gendered in a particular way varies from place to place, in different times, and is heavily influenced by race, age and other factors. As such, our paper should be taken as a first step, rather than as broadly applicable to 'gender' in its entirety. Moreover, even though our participants came from diverse educational levels and backgrounds, they represent a predominantly computer-literate population. Thus, further work may be required to ensure whether these observations can be generalised to a wider population. 
While within the community considered for this study (a Western country), the chosen topics and names were appropriate to elicit gender stereotypes (as evident by our quantitative and qualitative results), they may not generate similar results cross-culturally due to different cultural norms. Therefore, we encourage future research to extend our work by investigating different communities following a similar pilot test (as explained in Section 3.1) to ensure that chosen topics and names are in line with the gender perceptions of the targeted community.

Moreover, to exclude confounding variables such as participant assertiveness, we deployed our study in a controlled environment (one participants at a time). This is atypical for many online settings. We aim to explore the effect of synchronous interactions in an online setting in future work. Furthermore, the quiz we deployed utilised only objective questions as we aimed to investigate consequences of conformity in terms of answer correctness. Future research could expand our findings to subjective questions to explore the possible effects of gender-stereotypes on conformity.

\section{CONCLUSION}

Social conformity is a powerful social phenomenon in which individuals adjust their behaviour and opinions to agree with an opposing majority. While previous work has investigated conformity and its determinants in online group settings, the effects of gender and related stereotypes on online social conformity remain underexplored. This study investigated the effects of group gender composition and participant gender across both neutral and stereotypically perceived masculine (sports) and feminine (fashion) questions on conformity in an online quiz. We compared results across three conditions to evaluate the strength of different gender cues: a control condition where participants were oblivious to the gender of their peers; a condition displaying stereotypical masculine and feminine names of their peers; and finally a condition displaying peers using stereotypical masculine and feminine silhouette avatars. Our findings establish that individuals are receptive to subtle gender cues available in online group settings, and make (often incorrect) assumptions of their peers' competency based on prevailing gender-stereotypes, especially when making decisions in uncertain situations.

We conclude our work with a discussion on the implications of our findings in designing for online group settings, with minimum exposure to stereotypical gender biases. We suggest that designers carefully consider whether displaying gender and other user cues is relevant and value-adding from the perspective of end-users as well as the platform. We recommend against the use of gender cues such as binary-gendered avatars (especially in situations where group members could perceive the competency of others based on gender). Instead, we support using alternatives devoid of gender cues such as identicons, avatars with user initials, or site specific avatars, to ensure unbiased discussion and decision making. We encourage future work to explore similar effects on online social conformity, with regard to other aspects of a person's identity that can lead to stereotype-based conformity, such as age, race, and ethnicity.

\section{REFERENCES}

[1] Louie Andre. 2019. Finance Online: List of Top LMS Software Companies of 2019. https://financesonline.com/ top-20-lms-software-companies

[2] Solomon E Asch. 1951. Groups, leadership and men. Carnegie Press, Oxford, England, Chapter: Effects of group pressure upon the modification and distortion of judgements, 177-190.

[3] Solomon E Asch. 1955. Opinions and social pressure. Scientific American 193, 5 (1955), 31-35.

[4] Erin Ash. 2016. Priming or Proteus Effect? Examining the Effects of Avatar Race on In-Game Behavior and Post-Play Aggressive Cognition and Affect in Video Games. Games and Culture 11, 4 (2016), 422-440.

[5] April H. Bailey and Marianne LaFrance. 2016. Anonymously male: Social media avatar icons are implicitly male and resistant to change. Cyberpsychology 10, 4 (2016), 1 - 14.

[6] Susan A Banducci, Jeffrey A Karp, Michael Thrasher, and Colin Rallings. 2008. Ballot photographs as cues in low-information elections. Political Psychology 29, 6 (2008), 903-917.

[7] Andrew W Barrett and Lowell W Barrington. 2005. Is a picture worth a thousand words? Newspaper photographs and voter evaluations of political candidates. Harvard International fournal of Press/Politics 10, 4 (2005), 98-113.

[8] Douglas Bates, Martin Mächler, Ben Bolker, and Steve Walker. 2015. Fitting Linear Mixed-Effects Models Using lme4. Journal of Statistical Software 67, 1 (2015), 1-48. 
[9] Tanya Beran, Michelle Drefs, Alyshah Kaba, Noof Al Baz, and Nouf Al Harbi. 2015. Conformity of responses among graduate students in an online environment. The Internet and Higher Education 25 (2015), 63-69.

[10] Benjamin M. Bolker, Mollie E. Brooks, Connie J. Clark, Shane W. Geange, John R. Poulsen, M. Henry H. Stevens, and Jada-Simone S. White. 2009. Generalized linear mixed models: a practical guide for ecology and evolution. Trends in Ecology \& Evolution 24, 3 (2009), 127-135.

[11] Virginia Braun and Victoria Clarke. 2006. Using thematic analysis in psychology. Qualitative Research in Psychology 3, 2 (2006), 77-101.

[12] Samantha Breslin and Bimlesh Wadhwa. 2014. Exploring Nuanced Gender Perspectives Within the HCI Community. In Proceedings of the India HCI 2014 Conference on Human Computer Interaction (IndiaHCI '14). ACM, New York, NY, USA, Article 45, 10 pages.

[13] Jennifer D Campbell, Abraham Tesser, and Patricia J Fairey. 1986. Conformity and attention to the stimulus: Some temporal and contextual dynamics. Fournal of Personality and Social Psychology 51, 2 (1986), 315-324.

[14] Linda L Carli. 1989. Gender differences in interaction style and influence. fournal of Personality and Social Psychology 56, 4 (1989), 565-576.

[15] Emily Christofides, Towhidul Islam, and Serge Desmarais. 2009. Gender stereotyping over instant messenger: The effects of gender and context. Computers in Human Behavior 25, 4 (2009), 897-901.

[16] Robert B Cialdini. 2001. Harnessing the science of persuasion. Harvard Business Review 79, 9 (2001), $72-81$.

[17] Robert B Cialdini and Noah J Goldstein. 2004. Social influence: Compliance and conformity. Annu. Rev. Psychol. 55 (2004), 591-621.

[18] Marco Cinnirella and Ben Green. 2007. Does 'cyber-conformity' vary cross-culturally? Exploring the effect of culture and communication medium on social conformity. Computers in Human Behavior 23, 4 (2007), 2011-2025.

[19] Karen M Cornetto and Kristine L Nowak. 2006. Utilizing usernames for sex categorization in computer-mediated communication: Examining perceptions and accuracy. CyberPsychology \& Behavior 9, 4 (2006), 377-387.

[20] Morton Deutsch and Harold B Gerard. 1955. A study of normative and informational social influences upon individual judgment. The fournal of Abnormal and Social Psychology 51, 3 (1955), 629-636.

[21] Vitaly J Dubrovsky, Sara Kiesler, and Beheruz N Sethna. 1991. The equalization phenomenon: Status effects in computer-mediated and face-to-face decision-making groups. Human-Computer Interaction 6, 2 (1991), 119-146.

[22] Alice H Eagly. 1983. Gender and social influence: A social psychological analysis. American Psychologist 38, 9 (1983), 971-981.

[23] Alice H Eagly and Carole Chrvala. 1986. Sex differences in conformity: Status and gender role interpretations. Psychology of Women Quarterly 10, 3 (1986), 203-220.

[24] Alice H Eagly and Wendy Wood. 1985. Gender and influenceability: Stereotype versus behavior. In Women, gender, and social psychology. Erlbaum, Hillsdale, NJ, USA, 225-256.

[25] Susan Alizadeh Fard. 2010. Effect of gender and social status on conformity. Psychological Research 13, 1 (2010), 30 - 50.

[26] Katrina Fong and Raymond A Mar. 2015. What does my avatar say about me? Inferring personality from avatars. Personality and Social Psychology Bulletin 41, 2 (2015), 237-249.

[27] Heather Ford and Judy Wajcman. 2017. 'Anyone can edit', not everyone does: Wikipedia's infrastructure and the gender gap. Social studies of science 47, 4 (2017), 511-527.

[28] Jorge Goncalves, Vassilis Kostakos, and Jayant Venkatanathan. 2013. Narrowcasting in Social Media: Effects and Perceptions. In Proceedings of the 2013 IEEE/ACM International Conference on Advances in Social Networks Analysis and Mining (ASONAM '13). ACM, New York, NY, USA, 502-509.

[29] Victoria Groom, Jeremy N Bailenson, and Clifford Nass. 2009. The influence of racial embodiment on racial bias in immersive virtual environments. Social Influence 4, 3 (2009), 231-248.

[30] Joseph F Hair, William C Black, Barry J Babin, Rolph E Anderson, and RL Tatham. 2010. Multivariate Data Analysis. Pearson, New Jersey, NJ, USA.

[31] Eszter Hargittai and Steven Shafer. 2006. Differences in actual and perceived online skills: The role of gender. Social Science Quarterly 87, 2 (2006), 432-448.

[32] Libby Hemphill and Jahna Otterbacher. 2012. Learning the Lingo?: Gender, Prestige and Linguistic Adaptation in Review Communities. In Proceedings of the ACM 2012 Conference on Computer Supported Cooperative Work (CSCW '12). ACM, New York, NY, USA, 305-314.

[33] Chester A. Insko, Richard H. Smith, Mark D. Alicke, Joel Wade, and Sylvester Taylor. 1985. Conformity and Group Size: The Concern with Being Right and the Concern with Being Liked. Personality and Social Psychology Bulletin 11, 1(1985), 41-50.

[34] Richard A. Johnson and Gary I. Schulman. 1989. Gender-Role Composition and Role Entrapment in Decision-Making Groups. Gender and Society 3, 3 (1989), 355-372.

[35] Simon Kemp. 2019. Digital 2019: Global Internet Use Accelerates. https://wearesocial.com/blog/2019/01/ digital-2019-global-internet-use-accelerates

[36] Os Keyes. 2018. The Misgendering Machines: Trans/HCI Implications of Automatic Gender Recognition. Proc. ACM Hum.-Comput. Interact. 2, CSCW, Article 88 (Nov. 2018), 22 pages.

[37] Sara Kiesler, Jane Siegel, and Timothy W McGuire. 1984. Social psychological aspects of computer-mediated communication. American Psychologist 39, 10 (1984), 1123-1134. 
[38] Jitendra Kumar. 1983. Conformity behavior as a function of confederates' age and size of the confederate group. Personality Study \& Group Behaviour 3 (1983), 69-73.

[39] Lieve Laporte, Christof van Nimwegen, and Alex J. Uyttendaele. [n. d.]. Do People Say What They Think: Social Conformity Behavior in Varying Degrees of Online Social Presence. In Proceedings of the 6th Nordic Conference on Human-Computer Interaction: Extending Boundaries (NordiCHI '10). ACM, New York, NY, USA, 305-314.

[40] Eun-Ju Lee. 2003. Effects of 'gender' of the computer on informational social influence: the moderating role of task type. International fournal of Human-Computer Studies 58, 4 (2003), 347-362.

[41] Eun-Ju Lee. 2004. Effects of gendered character representation on person perception and informational social influence in computer-mediated communication. Computers in Human Behavior 20, 6 (2004), 779-799.

[42] Eun-Ju Lee. 2007. Wired for gender: Experientiality and gender-stereotyping in computer-mediated communication. Media Psychology 10, 2 (2007), 182-210.

[43] Eun-Ju Lee and Clifford Nass. 2002. Experimental tests of normative group influence and representation effects in computer-mediated communication: When interacting via computers differs from interacting with computers. Human Communication Research 28, 3 (2002), 349-381.

[44] John M Levine. 1999. Solomon Asch's legacy for group research. Personality and Social Psychology Review 3, 4 (1999), 358-364.

[45] Wendy Liu and Derek Ruths. 2013. What's in a name? Using first names as features for gender inference in twitter, In 2013 AAAI Spring Symposium Series. AAAI Spring Symposium - Technical Report SS-13-01, 10-16.

[46] Paul Benjamin Lowry, Tom L Roberts, Nicholas C Romano Jr, Paul D Cheney, and Ross T Hightower. 2006. The impact of group size and social presence on small-group communication: Does computer-mediated communication make a difference? Small Group Research 37, 6 (2006), 631-661.

[47] Michael Luca. 2017. Designing online marketplaces: Trust and reputation mechanisms. Innovation Policy and the Economy 17, 1 (2017), 77-93.

[48] Misa Maruyama, Scott P. Robertson, Sara Douglas, Roxanne Raine, and Bryan Semaan. 2017. Social Watching a Civic Broadcast: Understanding the Effects of Positive Feedback and Other Users' Opinions. In Proceedings of the 2017 ACM Conference on Computer Supported Cooperative Work and Social Computing (CSCW'17). ACM, New York, NY, USA, 794-807.

[49] Misa T. Maruyama, Scott P. Robertson, Sara K. Douglas, Bryan C. Semaan, and Heather A. Faucett. 2014. Hybrid Media Consumption: How Tweeting During a Televised Political Debate Influences the Vote Decision. In Proceedings of the 17th ACM Conference on Computer Supported Cooperative Work \& Social Computing (CSCW'14). ACM, New York, NY, USA, 1422-1432.

[50] Kimberly Matheson. 1991. Social cues in computer-mediated negotiations: Gender makes a difference. Computers in Human Behavior 7, 3 (1991), 137-145.

[51] Kimberly Matheson and Mark P Zanna. 1990. Computer-mediated communications: The focus is on me. Social Science Computer Review 8, 1 (1990), 1-12.

[52] Katelyn YA McKenna and Amie S Green. 2002. Virtual group dynamics. Group Dynamics: Theory, Research, and Practice 6, 1 (2002), 116-127.

[53] Kristine L Nowak and Christian Rauh. 2005. The influence of the avatar on online perceptions of anthropomorphism, androgyny, credibility, homophily, and attraction. fournal of Computer-Mediated Communication 11, 1 (2005), 153-178.

[54] Tom Postmes and Russell Spears. 2002. Behavior online: Does anonymous computer communication reduce gender inequality? Personality and Social Psychology Bulletin 28, 8 (2002), 1073-1083.

[55] Tom Postmes, Russell Spears, and Martin Lea. 2000. The formation of group norms in computer-mediated communication. Human Communication Research 26, 3 (2000), 341-371.

[56] Tom Postmes, Russell Spears, Khaled Sakhel, and Daphne De Groot. 2001. Social influence in computer-mediated communication: The effects of anonymity on group behavior. Personality and Social Psychology Bulletin 27, 10 (2001), 1243-1254.

[57] Stephen D Reicher, Russell Spears, and Tom Postmes. 1995. A social identity model of deindividuation phenomena. European Review of Social Psychology 6, 1 (1995), 161-198.

[58] Bernardo Reynolds, Jayant Venkatanathan, Jorge Gonçalves, and Vassilis Kostakos. 2011. Sharing Ephemeral Information in Online Social Networks: Privacy Perceptions and Behaviours. In Human-Computer Interaction - INTERACT 2011, Pedro Campos, Nicholas Graham, Joaquim Jorge, Nuno Nunes, Philippe Palanque, and Marco Winckler (Eds.). Springer Berlin Heidelberg, Berlin, Heidelberg, 204-215.

[59] Michael Rosander and Oskar Eriksson. 2012. Conformity on the Internet-The role of task difficulty and gender differences. Computers in Human Behavior 28, 5 (2012), 1587-1595.

[60] Leon A Rosenberg. 1963. Conformity as a function of confidence in self and confidence in partner. Human Relations 16, 2 (1963), 131-139.

[61] Victor Savicki, Merle Kelley, and E Oesterreich. 1999. Judgments of gender in computer-mediated communication. Computers in Human Behavior 15, 2 (1999), 185-194.

[62] Eva Sharma and Munmun De Choudhury. 2018. Mental Health Support and Its Relationship to Linguistic Accommodation in Online Communities. In Proceedings of the 2018 CHI Conference on Human Factors in Computing Systems (CHI'18). ACM, New York, NY, USA, Article 641, 13 pages. 
[63] David J Stang. 1976. Ineffective deception in conformity research: Some causes and consequences. European fournal of Social Psychology 6, 3 (1976), 353-367.

[64] Abhay Sukumaran, Stephanie Vezich, Melanie McHugh, and Clifford Nass. 2011. Normative Influences on Thoughtful Online Participation. In Proceedings of the SIGCHI Conference on Human Factors in Computing Systems (CHI '11). ACM, New York, NY, USA, 3401-3410.

[65] Abraham Tesser, Jennifer Campbell, and Susan Mickler. 1983. The role of social pressure, attention to the stimulus, and self-doubt in conformity. European fournal of Social Psychology 13, 3 (1983), 217-233.

[66] Paul Wallace and James Maryott. 2009. The impact of avatar self-representation on collaboration in virtual worlds. Innovate: fournal of Online Education 5, 5 (2009).

[67] Wendy Wood and Stephen J Karten. 1986. Sex differences in interaction style as a product of perceived sex differences in competence. Journal of Personality and Social Psychology 50, 2 (1986), 341-347.

[68] Seung-Chul Yoo, J.F. Pena, and M.E. Drumwright. 2015. Virtual shopping and unconscious persuasion: The priming effects of avatar age and consumers' age discrimination on purchasing and prosocial behaviors. Computers in Human Behavior 48 (2015), 62 - 71.

Received April 2019; revised June 2019; accepted August 2019 


\section{Chapter 7}

\section{Online Social Presence as a Determinant of Conformity}

\subsection{Introduction}

Social presence - i.e. the sense of being connected to others - is one of the most frequently investigated contextual determinants of conformity in both physical and CMC group settings (see Table 2.2). More importantly, it is the only determinant to be investigated more often in CMC groups than in physical groups (see Table 2.2). This is not surprising as social presence is more relevant in CMC groups - where it is a variable that can be controlled through platform design - in contrast to face-to-face groups where it is implicit to a great extent. Additionally, social presence is multi-faceted in nature and hence has been investigated across different aspects - including but not limited to - user representations [89, 103], interactivity [97, 111], response visibility [106] in CMC groups. However, these studies have focused on only one aspect of online social presence at a time, whereas in realistic online groups they are more likely to manifest simultaneously. Furthermore, as the level of perceived social presence moderates user receptiveness to social pressure [156], it is vital to investigate effects of online social presence alongside other contextual and personal conformity determinants.

In this chapter we extend prior work by investigating the compound effects of online user representations (generic vs. user-specific avatars to represent users), interactivity (discussion vs. no discussion after exposure to group answers) and response visibility (private vs. public final responses) - on user conformity behaviour in an online group quiz. Moreover, we investigate the impact of social presence in both objective and subjective quiz questions, as individuals are opposed and supported by majorities and minorities of different sizes, while also accounting for their confidence on personal answers - to quantify effects of social presence in relation to other conformity determinants.

Our results indicate a statistically significant interaction effect between the level of interactivity and response visibility on the platform, and no other main effects from the three aspects of social presence considered. More specifically, highest user conformity is observed in the presence of peer discussion (higher interactivity) and public responses (higher response visibility), and lowest in the absence of these two factors. Additionally, we note more frequent user conformity in objective than subjective quiz questions, when users indicate low confidence on initial answers, and are contradicted by larger than smaller majorities - corroborating findings of Articles I, II \& III.

Informed by our findings, we set forth several design implications - that we describe in detail in the attached publication, Article IV. We show that designers can manipulate the level of interactivity and the visibility of user responses on online group platforms 
to manage perceived online social presence - which consequently controls perceived conformity pressures. This allows platform designers to encourage conformity where desired (e.g. online support groups [154]) by increasing interactivity and visibility of user responses. Alternatively, they can also reduce perceived social presence - and hence susceptibility to social conformity pressures - by limiting interactivity and response visibility among users, in situations where adverse effects of conformity are likely (e.g. online learning platforms $[19,81])$.

\subsection{Article IV}

Copyright is held by the authors. Publication rights licensed to ACM. This is the authors' version of the work. It is posted here for your personal use. Not for redistribution. The definitive Version of Record was published in:

Wijenayake, S., Van Berkel, N., Kostakos, V., Goncalves, J. (2020). Quantifying the Effect of Social Presence on Online Social Conformity. Proceedings of the ACM on HumanComputer Interaction, 4(CSCW1), 1-22. https://doi.org/10.1145/3392863.

Ethics ID: 1954591, The University of Melbourne Human Ethics Advisory Group. 


\title{
Quantifying the Effect of Social Presence on Online Social Conformity
}

\author{
SENURI WIJENAYAKE, The University of Melbourne, Australia \\ NIELS VAN BERKEL, Aalborg University, Denmark \\ VASSILIS KOSTAKOS, The University of Melbourne, Australia \\ JORGE GONCALVES, The University of Melbourne, Australia
}

Social conformity occurs when individuals in group settings change their personal opinion to be in agreement
with the majority's position. While recent literature frequently reports on conformity in online group settings, the
causes for online conformity are yet to be fully understood. This study aims to understand how social presence i.e.,
the sense of being connected to others via mediated communication, influences conformity among individuals
placed in online groups while answering subjective and objective questions. Acknowledging its multifaceted
nature, we investigate three aspects of online social presence: user representation (generic vs. user-specific
avatars), interactivity (discussion vs. no discussion), and response visibility (public vs. private). Our results show an
overall conformity rate of 30\% and main effects from task objectivity, group size difference between the majority
and the minority, and self-confidence on personal answer. Furthermore, we observe an interaction effect between
interactivity and response visibility, such that conformity is highest in the presence of peer discussion and public
responses, and lowest when these two elements are absent. We conclude with a discussion on the implications
of our findings in designing online group settings, accounting for the effects of social presence on conformity.

CCS Concepts: $\bullet$ Human-centered computing $\rightarrow$ Empirical studies in collaborative and social computing; Empirical studies in HCI.

Additional Key Words and Phrases: Online Social Conformity; Online Social Presence; Interactivity; Response Visibility; User Representation; Majority Size; Task Objectivity; Self-confidence

ACM Reference Format:

Senuri Wijenayake, Niels van Berkel, Vassilis Kostakos, and Jorge Goncalves. 2020. Quantifying the Effect of Social Presence on Online Social Conformity. Proc. ACM Hum.-Comput. Interact. 4, CSCW1, Article 55 (May 2020), 22 pages. https://doi.org/10.1145/3392863

\section{INTRODUCTION}

Conformity is a widely observed social phenomenon where individuals adjust their personal opinions to be in line with the group's expectations in an attempt to be 'liked' within the group (normative influence) or to be considered 'right' (informational influence) $[2,16]$. While conformity was initially studied in face-to-face settings, understanding its mechanisms in online settings is becoming increasingly important, primarily given that a growing range of societal interactions are now mediated through online technologies [10,51].

Moreover, there is an increasing interest in online social conformity in the recent $\mathrm{HCI} / \mathrm{CSCW}$ literature. Individuals are seen to conform to contradicting opinions and norms set by majorities when

Authors' addresses: Senuri Wijenayake, swijenayake@student.unimelb.edu.au, The University of Melbourne, Parkville, VIC, 3010, Australia; Niels van Berkel, nielsvanberkel@cs.aau.dk, Aalborg University, Fredrik Bajers Vej 5, Aalborg, 9100, Denmark; Vassilis Kostakos, vassilis.kostakos@unimelb.edu.au, The University of Melbourne, Parkville, VIC, 3010, Australia; Jorge Goncalves, jorge.goncalves@unimelb.edu.au, The University of Melbourne, Parkville, VIC, 3010, Australia.

Permission to make digital or hard copies of all or part of this work for personal or classroom use is granted without fee provided that copies are not made or distributed for profit or commercial advantage and that copies bear this notice and the full citation on the first page. Copyrights for components of this work owned by others than the author(s) must be honored. Abstracting with credit is permitted. To copy otherwise, or republish, to post on servers or to redistribute to lists, requires prior specific permission and/or a fee. Request permissions from permissions@acm.org.

(C) 2020 Copyright held by the owner/author(s). Publication rights licensed to ACM.

2573-0142/2020/5-ART55 \$15.00

https://doi.org/10.1145/3392863

Proc. ACM Hum.-Comput. Interact., Vol. 4, No. CSCW1, Article 55. Publication date: May 2020. 
discussing political and social issues on social media [43, 44], when completing online quizzes [6,67] and visual judgement tasks [29], and when commenting on online news websites [60]. However, limited research has been undertaken to understand what triggers conformity in online settings. Furthermore, as online groups are inherently dissimilar to face-to-face groups due to reduced social contextual cues and the lack of physicality in online communication [45], previous work on wellunderstood determinants of face-to-face conformity (such as majority group size [3,30,54], task objectivity (objective or subjective nature of the task) [2,7,17], and self-confidence [11,61]), may not be of relevance in online group settings. Thus, further work is required to systematically investigate whether and how aforementioned determinants of face-to-face conformity manifest in online group settings, as an initial step towards fully understanding online social conformity.

Furthermore, as conformity is a form of social influence, the effect of social presence i.e., the awareness of and the sense of being connected to others via mediated communication [58], on online conformity behaviour has been an interest in recent literature [33, 37, 47]. However, the aforementioned studies limit their focus to only one aspect of online social presence (such as user representation [47], level of interactivity [33] or response visibility [37]), where as in realistic online settings these aspects are more likely to manifest together (e.g., online discussions usually involve certain user representations). Thus, in this study, we contribute to the existing literature by investigating the compound effects of three aspects of online social presence - user representation (generic vs. user-specific avatars), interactivity (discussion vs. no discussion) and response visibility (public vs. private responses) - on online conformity behaviour. Moreover, we investigate the effect of social presence in both objective and subjective quiz tasks, as individuals are challenged by majorities of different group sizes, while also accounting for their level of self-confidence to provide a wider understanding of online conformity determinants in comparison to existing work.

Our results reveal main effects from task objectivity, group size difference (difference between the majority group size and the minority group size), and initial self-confidence of the participant. We observe higher conformity in objective questions than in subjective questions, contradicting the findings of offline social conformity [7], likely due to the inherently lower social presence in online settings. We also note that larger majorities are more influential than smaller ones as previously suggested by both offline [3, 20,54] and online [42, 65, 67] conformity literature. Moreover, participants are more likely to conform to the majority when they are less confident or uncertain of their initial answers, expanding the implications of existing literature on self-confidence and conformity in face-to-face groups $[11,61]$ to online settings.

While we do not observe main effects for the three aspects of social presence manipulated in this study, we note a statistically significant interaction effect between interactivity and response visibility. Conformity peaks in the presence of peer discussion and public responses, and remains at nominal levels in their absence. Thus, our findings imply that in addition to well-known conformity determinants such as majority size, task objectivity, and self-confidence, the level of social presence facilitated in an online setting plays a major role in users' conformity behaviour, making them especially susceptible to normative influences. We further discuss how this may be desired in settings where encouraging group norms is important (e.g., online support groups [56, 57]), while it may have detrimental effects when conformity is not desired (e.g., online learning environments [6]). Thus, our findings should be taken into consideration when designing for online group settings in order to promote positive interactions.

\section{RELATED WORK}

In 1955, Asch set out to investigate the effect of peer opinions on individual judgements in face-toface group settings [3]. He employed a simple 'line matching' experiment, where for a significant number of responses (36.8\%) participants conformed to a clearly incorrect yet unanimous majority, establishing the susceptibility of individuals in group settings to conformity influences. Subsequently, Deutsch and Gerard [16] replicated Asch's experiment to reveal two motives behind group conformity: normative and informational influences. They described that individuals conform to the majority

Proc. ACM Hum.-Comput. Interact., Vol. 4, No. CSCW1, Article 55. Publication date: May 2020. 
either with the intention of agreeing to their positive expectations (normative influence) or because they perceive information obtained from others to be more accurate evidence of a given situation than their own knowledge (informational influence). The notion of normative influence was further explained in $[14,38]$ as the inclination to ensure one's membership in a group, and seeking direction from the majority in uncertain situations as a manifestation of informational influences [13, 38].

Even though seminal social conformity literature is based on offline groups, owing to the recent movement of human societal interactions towards online platforms (e.g., social networks, learning platforms, discussion forums, support groups) [10, 21, 40, 51, 63], a growing number of $\mathrm{HCI} / \mathrm{CSCW}$ researchers have sought to investigate the manifestation and implications of social conformity on online group settings as described below.

\subsection{Online Social Conformity}

Recent literature suggest that social conformity has diverse manifestations in a wide variety of online settings [6, 29, 43, 44, 56, 60, 69]. For instance, a study by Zhu et al. [69] investigated how individuals adjust their online choices when challenged by recommendations from other users. The study required participants to chose between two options, with and without the knowledge of others' preferences. They observed that an individual's choices in an online setting can be significantly swayed by others' opposing recommendations. Similarly, Maruyama et al. [43] note that people tend to adopt the majority's opinions when discussing social issues on online social networks, even when they are unaware of the users who are posting the content. Another study highlighted that users who were actively involved in Twitter during a televised political debate were more inclined to adjust their voting choice to support the majority sentiment on Twitter, further establishing the presence of social conformity in online settings [44].

Literature also provides evidence of the negative consequences of social conformity in certain online settings. For instance, Beran et al. [6] note that students who saw peer answers when completing an online quiz frequently conformed to the majority's answers, and obtained fewer correct answers compared to students who answered the quiz independently. A similar study by Hullman et al. [29] further emphasised the effect of social information (i.e., the notion that we tend to believe things more when we see others doing them) on the accuracy of a simple visual judgement task completed by Mechanical Turk users. They highlight that seeing biased and incorrect social information led to more errors, which could eventually nullify the expected benefits of collective intelligence.

However, online social conformity is not without its potential benefits. For example, Sukumaran et al. [60] highlight how normative influences can be used in online news websites to encourage high quality and 'thoughtful' contributions from its users. They note that when initial comments are of high quality, subsequent participants were also encouraged to contribute with similar effort. Similarly, previous work has shown that conforming to the acceptable conventions of behaviour and linguistic norms improved the sense of belonging and security within an online mental health support group, so that sensitive issues could be openly discussed [56].

The aforementioned literature indicates both positive and negative implications of online social conformity. We therefore argue that a thorough understanding of factors influencing this social phenomenon is critical to ensure that future online platforms facilitating social interactions are designed accounting for possible conformity effects. Therefore, we next summarise the existing literature on major determinants of social conformity as seen in both offline and online settings.

\subsection{Determinants of Social Conformity}

2.2.1 Majority Size. The majority group size (or the number of influential sources) on conformity behaviour has been popularly researched in offline groups and several theories have been put forward. For instance, Asch [3] observed that against a minority of one, the influential power of the majority increased until its third member, while adding a fourth member to the majority did not increase its conformity influence. Alternatively, Latané [34] proposed that while larger majorities have greater

Proc. ACM Hum.-Comput. Interact., Vol. 4, No. CSCW1, Article 55. Publication date: May 2020. 
impact, the added impact is smaller for each additional group member. Similarly, the notion that larger majorities lead to higher conformity was further confirmed in subsequent studies $[30,46,54]$. However, the above studies considered unanimous majorities against a minority of one (participant) and did not account for the possibility of having larger minorities - which is typically the case in realistic online group settings.

Furthermore, a study by Lowry et al. [42] compared conformity behaviour across two group sizes (groups of three and six members) in both face-to-face and computer-mediated communication (CMC). They highlight that while conformity effects heightened in both conditions as the majority group size increased, the effect was lowered in the computer-mediated condition. Moreover, Walther et al. [65] investigated the effects of majority and minority group sizes on conformity in CMC group settings. Interestingly, their results show that the presence of minorities disturbing the unanimity of the group reduce the impact of the majority group size on conformity. However, while the significance of majority and minority group sizes on conformity behaviour has been suggested in the current literature, it is yet to be thoroughly investigated in an online setting.

2.2.2 Task Objectivity. Literature suggests that task objectivity can also play a significant impact on conformity behaviour. While conformity was initially tested in tasks of objective nature with an obvious correct answer [3,16], researchers were later interested in investigating how conformity manifests in tasks of subjective nature. For instance, Ferguson [17] observed conformity in tasks of attitudinal nature. Subsequently, Blake et al. [7] compared conformity effects across objective and subjective tasks. The authors note that higher conformity was observed in subjective tasks in comparison to objective tasks, as a result of higher normative influences in physical groups. They highlight that the motivation to achieve correct answers may have outweighed the appeal of conforming to an incorrect majority in objective questions. However, further work is required to understand whether and how objective and subjective questions may differ in eliciting conformity behaviour in online settings.

2.2.3 Self-confidence. Several studies have also investigated the effect of participant confidence on conformity behaviour $[11,61]$. Their results in unison emphasise a negative relationship between self-confidence and conformity [11, 61]. This notion is in line with Deutsch and Gerard's view of informational influences, which suggests that individuals conform to the majority seeking the 'correct' response in uncertain situations [16]. However, the impact of self-confidence on social conformity is yet to be understood in online group settings.

2.2.4 Social Presence. Social presence has been described in the literature as the the awareness of and the sense of being connected to others via mediated communication [58]. It has also been defined as the ability of individuals to project themselves socially and emotionally as 'real' people in mediated communication [19, 23]. Furthermore, Short et al. [58] explained that social presence facilitated by a medium depends on its ability to convey the presence of the communicators through verbal and non-verbal cues. Despite the early perception that CMC is impersonal due to the absence of social context cues, results of subsequent studies contradicted this notion [23, 24, 62]. These studies further emphasised that online social presence is a complex, multifaceted concept which manifests itself across multiple dimensions (e.g., social context cues, interactivity and privacy), each with its own variables (e.g., interactivity could be measured in terms of timely responses, communication style, formality of language etc.) [24, 62].

Subsequently, a study exploring the impact of different communication mediums (e.g., face-to-face, telephone, chat, and email) on perceived social presence and interpersonal perceptions, identified that people are more likely to behave in a manner to be liked by others in a richer communication medium eliciting higher social presence [15]. More recent literature has built on this notion to understand the impact of different aspects of online social presence (such as user representation, interactivity, and response visibility) on social conformity. For example, previous work has observed that online user representations (a social context cue) with more anthropomorphic (human-like) features encouraged higher social attractiveness and trustworthiness of online interaction partners, leading to higher

Proc. ACM Hum.-Comput. Interact., Vol. 4, No. CSCW1, Article 55. Publication date: May 2020. 
conformity in online group settings [22,37]. This behaviour was further explained in work by Nowak and Biocca [47], describing how the agency (the perception of communicating with a computer agent or a human being) and anthropomorphism offered by online user representations could significantly influence users' perceptions of social presence. Researchers highlight that participants reported higher social presence when representations were more realistic, human-like (high anthropomorphic) and perceived to represent a real human being. However, the online user representations used in the above studies were stereotypically gendered (which may also pose a significant impact on online conformity behaviour [35, 36, 67]) and are outdated (e.g., [37] compared text boxes against, stick figures and animated characters). Thus, further work is needed to explore the generalisability of these observations to more modern user representations.

Interactivity is another major dimension of online social presence [62], and has been investigated with regard to online social conformity. For example, Laporte et al. [33] compared conformity behaviour in participants answering an online quiz under two conditions: in a unidirectional setting where participants could see the answers of others alongside their profile pictures (no interaction), and in a bidirectional setting where participants were connected through a live video chat, capable of freely communicating with each other. They note higher conformity among participants in the live video condition, confirming the notion that individuals are more likely to conform in online settings with higher interactivity. Moreover, literature also supports the notion that online discussions could elicit both normative and informational influences leading to changes in an individual's opinions [49].

Furthermore, the impact of response visibility (i.e., whether user responses are visible to others or not) has been an interest within conformity research. The literature notes that conformity is considerably higher when users are informed that their responses are visible to the group (public) in comparison to situations where user responses are private [37]. Deutsch and Gerard [16] explained that being aware of others in public conditions leads to participants being susceptible to normative influences, in addition to the informational influences observed in private conditions.

While the impact of user representation, interactivity, and response visibility on online social conformity has been investigated independently, existing work does not account for their interaction effects, despite their likelihood to manifest simultaneously in realistic online settings. Thus, further work is required to investigate the impact of their interaction effects on online conformity. Moreover, it is possible that the effect of social presence on online conformity also depends on other well-known determinants such as majority group size, task objectivity and self-confidence, which is yet to be thoroughly investigated. Thus, in this study we aim to systematically examine how the aforementioned aspects of social presence impact online conformity behaviour in both objective and subjective tasks, with different majority - minority group compositions, while also accounting for effects of self-confidence.

\section{METHOD}

To investigate the effect of social presence on conformity behaviour in an online setting, we manipulate the level of social presence in terms of user representation (generic vs. user-specific avatars), interactivity (discussion vs. no discussion), and response visibility (public vs. private responses). To control these variables of online social presence, while simulating a plausible online group setting where users are required to make judgements, we deployed an online multiple choice questions (MCQ) quiz containing both subjective and objective questions. MCQ quizzes are frequently used in online social conformity experiments $[6,33,53,67,68]$ as they enable the simulation of a clear group majority and a minority while placing the participant in both these groups.

\subsection{The Quiz}

The online quiz contained 18 multiple choice questions ( 9 objective and 9 subjective questions). The objective questions were extracted from both existing literature [67] and popular online question repositories such as Sporcle and Britannica, which have been previously used in $[67,68]$ to extract quiz

Proc. ACM Hum.-Comput. Interact., Vol. 4, No. CSCW1, Article 55. Publication date: May 2020. 


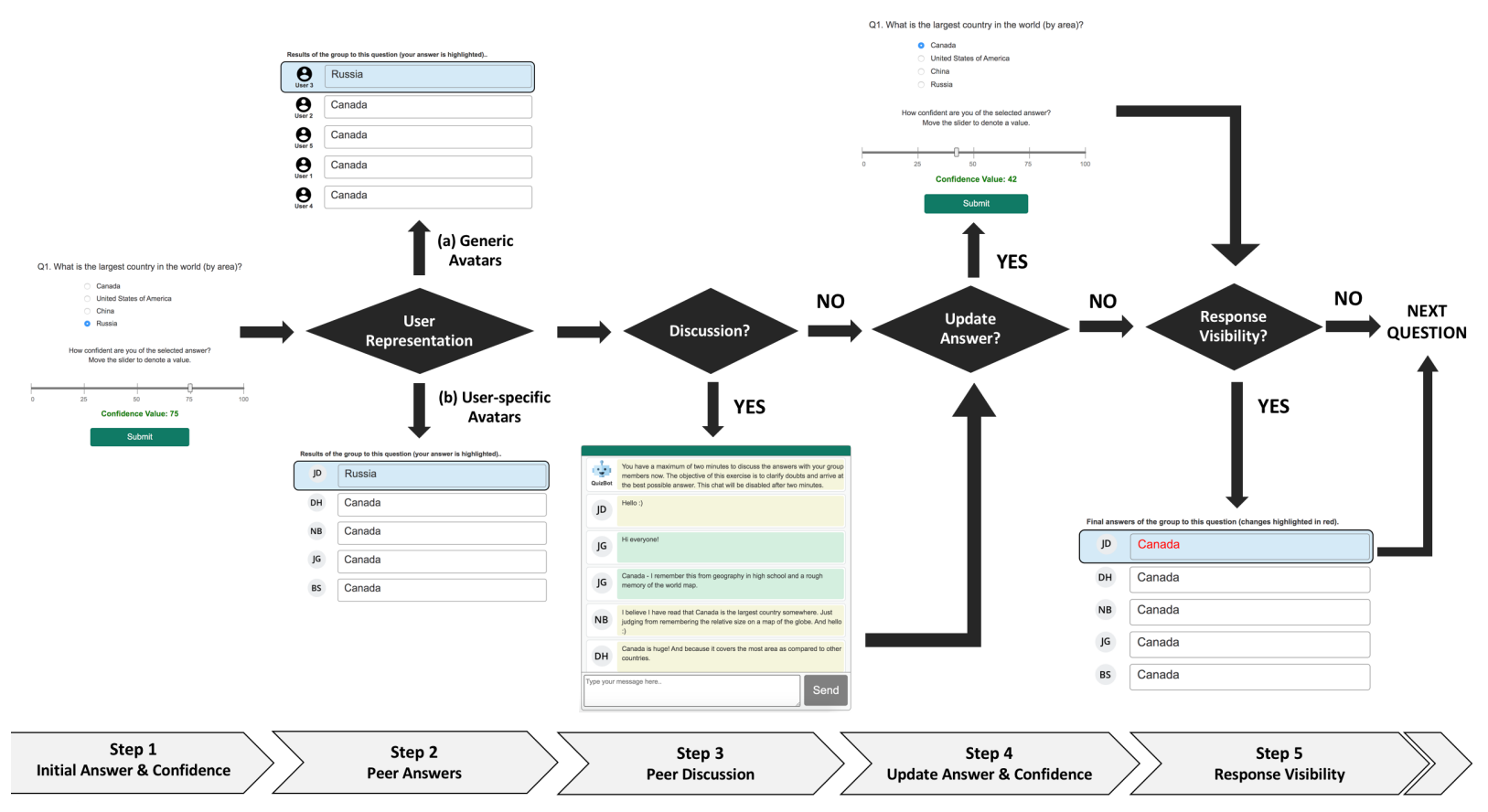

Fig. 1. Steps followed during the quiz by participants. Step 1: Initial answer and confidence, Step 2: Peer answers, Step 3: Peer discussion, Step 4: Update answer and confidence, Step 5: Response visibility

questions. This ensured that questions were only included if they were based on topics considered as general knowledge within the community considered for this experiment. Previous work investigating online social conformity through quizzes have also utilised general knowledge topics in their experiments $[33,53,67,68]$. The subjective questions were extracted from an article in ThoughtCo. outlining debating topics for high school students. We ensured that no overly sensitive topics were discussed in the quiz. A complete list of questions utilised in the quiz is included as supplementary material.

Participants followed the structure illustrated in Fig. 1 to complete the quiz. First, the participant is instructed to select their personal answer and rate their confidence on the chosen answer as illustrated in Step 1 of Fig. 1. Self-reported confidence levels were denoted using a scale ranging from $0-100$ with higher values representing higher levels of confidence. Subsequent to submitting their initial answer and confidence, the participant is shown a fabricated list of peer answers as chosen by four other participants completing the quiz (along with either generic or user-specific avatars as shown in Step 2(a) and Step 2(b) in Fig. 1). The fabricated peer answers were dynamically generated by our software to show the distribution of votes from other participants across a clear majority and a minority, while placing the participant in either group. The participant's answer was highlighted for convenience. This notion of using fabricated peer answers to investigate online social conformity was motivated by recent literature on conformity $[53,67,68]$. The next step determined the level of interactivity (discussion vs. no discussion) facilitated during the study. Participants in discussion conditions were given two minutes to discuss the group answers and their justifications with 'peers' through a real-time and text-based group chat as shown in Step 3 of Fig. 1. In reality, confederates of the researchers were used to simulate 'peer' discussions based on a predetermined script. Subsequently, all users were given the opportunity to change their answer option and/or confidence to finalise their answer (Step 4). Next, the response visibility (i.e., whether or not the final answers are visible to the rest of the group) was manipulated as shown in Step 5 of Fig. 1. Participants in public conditions were shown the final answers of the group with changed answers highlighted in red. After viewing the group's final answers, the user is taken to the next question. 


\subsection{Group Composition}

We chose an overall group size of five users (i.e., the size of the majority and the minority sums up to five) to investigate the effect of different majority - minority group distributions as illustrated in Fig. 2. While participants were informed that they will be connected with four peers to complete the quiz, there was only one real participant completing the quiz at any given time. Previous work investigating social conformity in both face-to-face and online groups has employed a similar group size [7, 16, 30, 37].

During the quiz, group answers were fabricated such that the participant was evenly placed in the majority (see group compositions (b) and (c) in Fig. 2) and in the minority group (see group compositions (d) and (e) in Fig. 2). We also simulated group consensus (in two questions) to provide a sense of authenticity to the peer answers. Moreover, we ensured that each group combination was equally tested against topics of both objective and subjective nature.

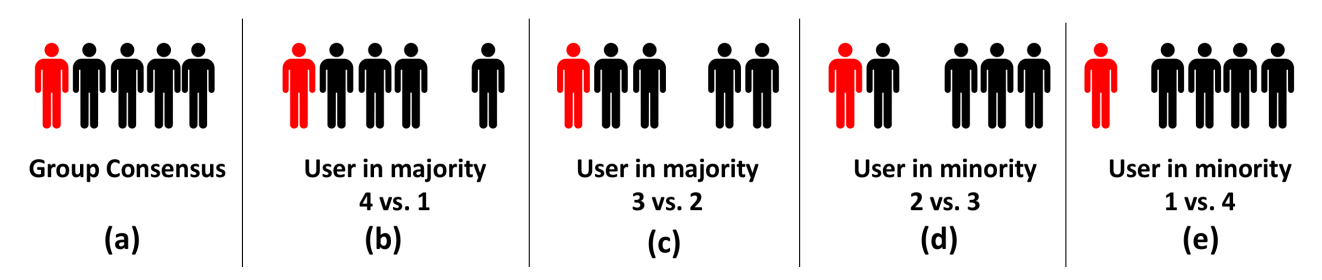

Fig. 2. Overview of group compositions investigated in the quiz (participant highlighted in red).

\subsection{Social Presence}

The main objective of this study was to investigate the compound effects of online social presence amidst other well-known determinants of conformity. Thus, we manipulated online social presence using three variables - user representation (generic vs. user-specific avatars), interactivity (discussion vs. no discussion) and response visibility (public vs. private responses) - which resulted in a $2 \times 2 \times$ 2 experimental design (8 conditions). Participants of each experimental condition interacted with a unique combination of the aforementioned interface elements. For example, participants assigned to the generic $\mathrm{x}$ discussion $\mathrm{x}$ public condition were represented using a single generic avatar, discussed answers with peers via a group chat, and saw final group answers, whereas participants in the user-specific $\mathrm{x}$ no discussion $\mathrm{x}$ private condition were denoted using user-specific avatars and did not see a group chat or final group answers. We now describe these experimental conditions in detail.

3.3.1 User Representation. User representations are important social context cues contributing to the perceived social presence in online settings [62]. In this study we investigate the impact of two commonly used online user representations: generic and user-specific avatars. In generic avatar conditions, a commonly used gender-neutral avatar is assigned to all the five users along with generic usernames such as "User 1" and "User 2" to differentiate between participants (see Step 2(a) in Fig. 1). Alternatively, in the user-specific avatar conditions, users are assigned dynamically generated avatars including the first letters of their first and last names (e.g., John Doe is represented by JD as shown in Step 2(b) in Fig. 1).

We highlight that our choice of user representations is based on literature explaining how agency and anthropomorphism associated with different online user representations could impact social presence in virtual group settings [22, 37, 47]. Based on the evidence provided in literature we hypothesise that user-specific avatars with user initials convey a stronger sense of being connected to a 'real' human being, than a single generic avatar with computer generated usernames. Moreover, the practice of assigning a default generic avatar to users is common in online social networks such as Twitter and YouTube and even in Learning Management Systems such as SAP Litmos and Docebo, where user decisions are likely to be influenced by others. Alternatively, some popular online 
platforms such as Google use user-specific avatars with user initials to represent them. The user representations used in this study are purposefully devoid of explicit user cues (such as gender, name, or age) to avoid confounding effects on online social conformity [12, 32, 35, 36, 39, 67].

3.3.2 Interactivity. We consider two levels of interactivity in this study: discussion and no discussion. This determines whether participants are given an opportunity to engage in a discussion with peers after viewing their answers (i.e., discussion) or not (i.e., no discussion).

In discussion conditions, users are informed that after being shown the peer answers, they will be given two minutes to discuss their answers and rationale behind selecting it, with others in the group as shown in Step 3 of Fig. 1. However, as we recruited only one participant per session, four confederates of the researchers pretended to be users participating in the quiz, to simulate a group discussion. The confederates engaged in the group discussion based on a script, dynamically created by the software for each question based on the fabricated answer distribution. Confederates have been used to simulate real users in previous work investigating social conformity in both face-toface $[7,16,30]$ and online [33] group settings. Alternatively, in no discussion conditions, participants are only shown the fabricated answer list of the group members. They are not subsequently given an opportunity to engage in a group discussion, and are directly taken to Step 4 of Fig. 1.

3.3.3 Response Visibility. After the peer answers are shown, participants are given the opportunity to update their answers and/or confidence prior to submitting their final answers (see Step 4 of Fig. 1). Response visibility determines whether the final answers are visible to the group (i.e., public) or not (i.e., private). Participants in the public conditions are informed prior to the quiz that their final answers will be visible to the group. Therefore, they are shown the list of updated answers of the group before moving to the next question, with updated answers highlighted in red as shown in Step 5 of Fig. 1. Moreover, to ensure that participants in public conditions are not suspicious of the authenticity of the answers, in $50 \%$ of the questions where at least one other simulated participant was placed in the minority with or without the user (e.g., see (b), (c) and (d) in Fig. 2), the said simulated participant changed their answer to that of the majority.

Alternatively, participants in private conditions are told that their final answers will not be visible to others in their group, and are taken to the next question upon submitting their final answers.

\subsection{Participants and Procedure}

We recruited 64 participants (32 men and 32 women) from different educational backgrounds which included engineering, science, marketing, management, arts and architecture fields. All participants were between 19 - 36 years of age and were recruited through our university's online notice board. Participants were equally distributed among the 8 experimental conditions with an equal number of men and women participating in each condition.

The study was conducted in a laboratory with one participant per session, under the supervision of a researcher. Participants were informed that they would be taking part in an online quiz together with four other participants. As the true purpose of the study could not be disclosed prior to the quiz as expected in studies investigating conformity behaviour [59], we explained that the study was motivated by the increasing use of online learning platforms and that we intend to investigate the performance of students in online quizzes. No other information triggering a competing or cooperative relationship between the participants was provided.

Before starting the quiz, participants completed an online form which collected their self-disclosed gender, age, and educational background. Upon submitting their demographic details, participants were randomly assigned to an experimental condition. The steps followed by the participant, and the interface they interacted with, depended on the condition they were assigned to as illustrated in Fig. 1. For instance, only participants assigned to discussion conditions, were shown an online group chat area and were greeted by a conversational agent named 'QuizBot'. This chat area was not visible to participants in no discussion conditions. Alternatively, participants in no discussion conditions

Proc. ACM Hum.-Comput. Interact., Vol. 4, No. CSCW1, Article 55. Publication date: May 2020. 
were given textual, step-by-step instructions through the study software (with no involvement of the researchers) prior to initiating the quiz.

The 'QuizBot' was utilised in discussion conditions to regulate group discussions (see Fig. 3) without the involvement of a researcher. The absence of a researcher closely replicates realistic online group settings, while also reducing the Hawthorne effect, a crucial aspect in conformity research $[1,66]$. The bot also signalled the participants when to start the discussion after viewing the peer answers and displayed the remaining time left for discussion, to ensure that group discussions were restricted to the allocated time frame of two minutes per question. The group chat was automatically disabled by the bot after the allocated time, and the users were prompted to the next step. Furthermore, the 'QuizBot' provided confederates regular updates on what the participant was doing before and after the discussion (i.e., answering the question, changing the answer after the discussion etc.), displayed the fabricated list of peer answers, and provided them prompts of the discussion points based on a predetermined script to minimise confusion and error.

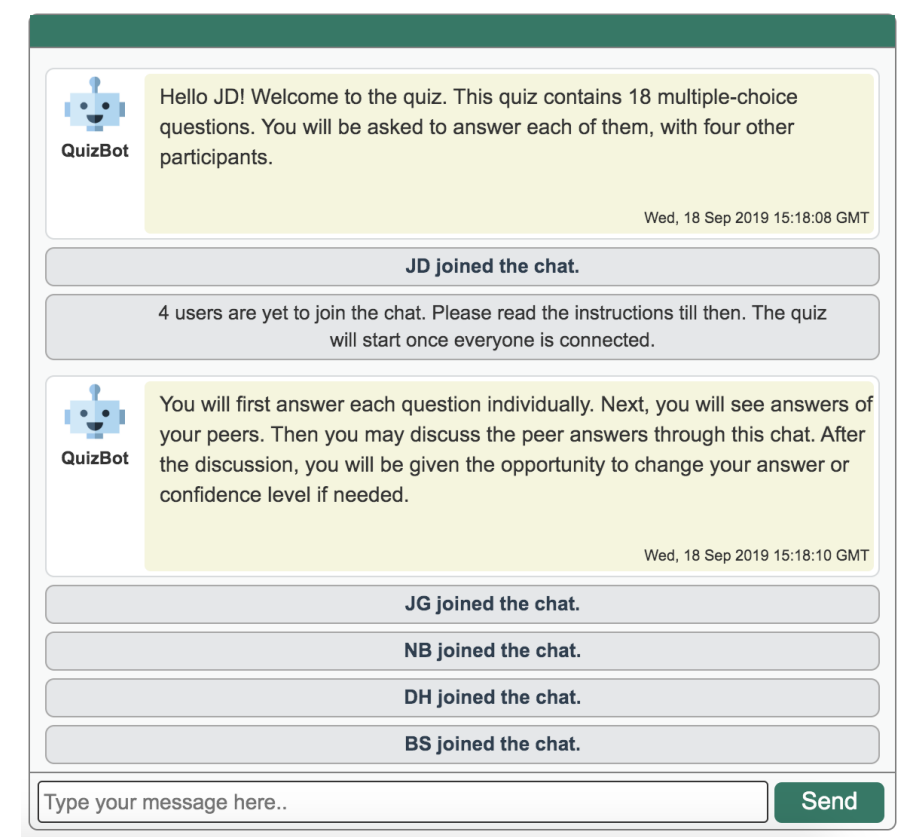

Fig. 3. QuizBot providing initial quiz instructions and informing participant status in the discussion conditions.

Upon completion of the quiz, participants participated in a brief semi-structured interview. The interview was arranged as follows. First, participants were asked for general thoughts on their experience participating in the study, to identify if they were suspicious of the authenticity of other participants. Subsequently, we inquired whether they changed their answers during the quiz and what factors contributed towards such behaviour. We were also interested in whether they were more inclined to change their answers in certain types of questions, to understand how task objectivity may affect their behaviour. Participants were also questioned about the impact of discussion, response visibility and user representations they were exposed to, to determine whether and how these three aspects of online social presence affected their conformity behaviour. Subsequently, we debriefed our participants on the true objective of the study. Participants were then given the opportunity to withdraw their participation and data collected during the study, if desired. All our participants consented to the use of the data collected, even upon revealing the study's true objective.

The experimental design was approved by the Ethics Committee of our university. The experiment lasted for approximately $60-90$ minutes per participant depending on the experimental condition they were assigned to, including briefing, completing the quiz, and the final interview. Each participant received a $\$ 20$ gift voucher for participation. 


\subsection{Preliminary Study}

Prior to the experiment, we conducted a preliminary study with 10 men and 10 women, where participants were asked to answer the same set of MCQs along with justifications for their answers. We obtained a total of 360 responses from the preliminary study. Next, we arranged the four answer options in each MCQ based on a descending order of the number of votes they received during the preliminary study, to determine the most popularly chosen answers for each question. This order was later considered along with the answers chosen by the participants in the main experiment, to dynamically determine the positioning of the majority and the minority groups when fabricating peer answers. For instance, in the question shown in Fig. 1 ("What is the largest country in the world (by area)?"), "Russia" and "Canada" were the top two answers chosen by the preliminary study participants. Thus, when a participant in the main study selected "Russia" as their initial answer, our software dynamically fabricated the peer answers placing "Canada" in a clear majority. The same approach was used to decide the minority's answer, when participants were placed in the majority. This ensured that the majority or the minority was always placed in a plausible answer option, regardless of being correct or incorrect.

Moreover, the justifications provided by participants in the preliminary study were used to create a script which was used by confederates to support their answers during the main experiment (in discussion conditions). This ensured that all justifications provided by the confederates simulating participants, closely represented how the considered community perceived the topic in question. This was especially crucial in the subjective questions. For objective questions, we chose justifications that could be used with all four of the answer options (e.g., "Canada/Russia/China/USA is huge! I also remember this fact from my geography class in high school."), and were counterbalanced among the four options during the experiment. We ensured that the justifications used in the final script did not include any obvious personal cues (such as gender or age) which could result in confounding effects on social conformity $[12,32,55]$. None of these explanations were explicit on the confidence level of the 'participant', or displayed uncertainty in the chosen answer. For instance, in the question shown in Fig. 1 the explanations provided by confederates could be as follows:

Confederate 1: "I remember from the world map that Canada is the biggest country in the world." Confederate 2: "Canada is huge! And because it covers the most area as compared to other countries."

Confederate 3: "I have read about this. Also by judging from remembering the relative size of Canada on a map of the globe."

Confederate 3: "I remember this as Canada from geography in high school and a rough memory of the world map."

\section{RESULTS}

All 64 participants answered 18 questions each, which resulted in a total of 1152 responses. Of these, participants were placed in the minority in 512 responses and in the majority or in a consensus in 640 responses (equally distributed among objective and subjective questions), to avoid drawing suspicion to the plausibility of the fabricated peer answers. On that note, we emphasise that our intention was not to compare results between majority and minority responses, but rather to investigate the impact of social presence on conformity across a diverse range of group compositions.

Upon displaying the fabricated peer feedback, participants were given the opportunity to:

- Change both initial answer option and confidence level.

- Change only their initial answer option.

- Change only their initial confidence level.

- Make no change to their initial answer.

Participants changed their initial answer and/or confidence at least once during the quiz, resulting in a total 431 changed responses with an average of 6.7 changes $(S D=4.1)$ per participant. Fig. 4 illustrates the distribution of the participants' post-feedback responses, when their answer was supported by

Proc. ACM Hum.-Comput. Interact., Vol. 4, No. CSCW1, Article 55. Publication date: May 2020. 
a minority, or by a majority during the quiz. Participants were more inclined to change one's answer (with or without a change in confidence) when placed in a minority (in approximately $34 \%$ of the minority responses). On the other hand, being placed in the group majority was more likely to result in an increase in participant's self-confidence on selected answer (in approximately $28 \%$ responses). Thus, these results establish that participants changed their answers post feedback, not randomly but due to the influence of the predictors we considered, confirming the validity of our results.

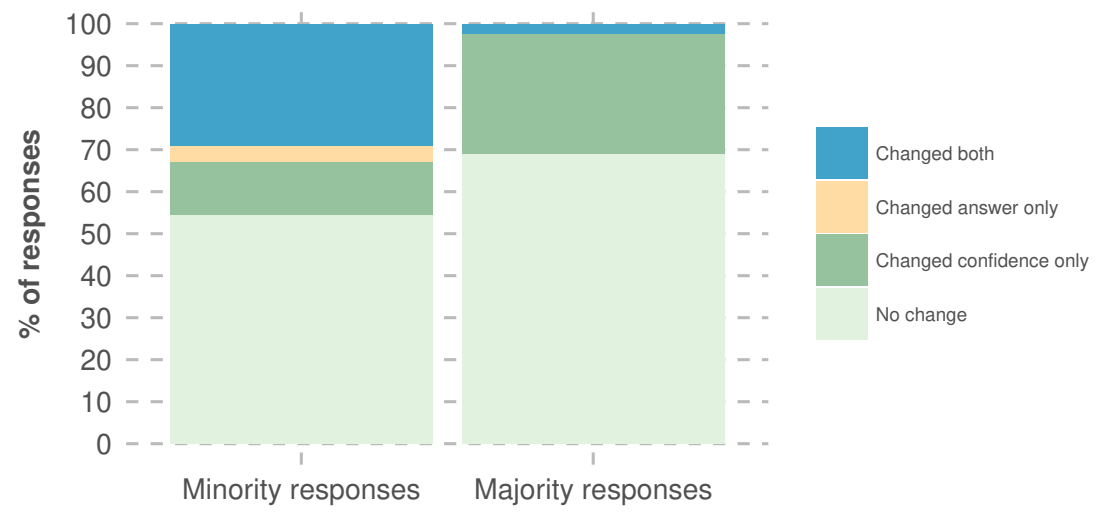

Fig. 4. Distribution of minority and majority responses across the four post-feedback response types.

\subsection{Model Construction}

For the purpose of this study, we define conformity as the act of changing one's answer to that of the majority. Our results show that 55 participants conformed at least once during the quiz, resulting in 152 conformity responses (approximately 30\% conformity), with an average of 2.4 conformity responses $(S D=1.6)$ per participant. We then investigated the impact of the following ten predictor variables on the conformity behaviour of our participants. For our model construction, we only consider the responses of participants when placed in a minority, as the dependent variable was determining conformity behaviour.

- Majority size: Size of the majority (possible values: 3 or 4).

- Minority size: Size of the minority (possible values: 2 or 1 ).

- Group size difference: Difference between the majority group size and the minority group size (possible values : 1 or 3 ).

- User representation: The avatar used to represent users in the online platform (possible values: generic or user-specific).

- Interactivity: Whether or not users were given an opportunity to discuss peer answers with the group (possible values: discussion or no discussion).

- Response visibility: Whether or not the final group answers were visible to others (possible values: public or private).

- Task objectivity: Subjective or objective nature of the question.

- Initial confidence: Participant's confidence in answer prior to revealing peer answers (ranging from 0 to 100).

- Gender: Participant's self-disclosed gender.

- User ID: An unique identifier assigned to a given user during the quiz.

We used the R package lme4 [5] to perform a generalised linear mixed-effects model (GLMM) analysis of the relationship between the aforementioned variables and participant conformity. A GLMM allows us to identify the effect of a set of predictor variables on an outcome variable (conformity) while following an arbitrary (i.e., possibly non-normal) distribution. We specified participant (User ID) as a random effect to account for individual differences in our model. 
All statistically significant predictors included in the final model (following model selection through incremental removal of variables based on their predictive power) are shown in Table 1. We perform a likelihood ratio test with the null model [8] and find that our model is statistically significant $\left(\chi^{2}=188.98, \mathrm{p}<0.001\right)$ and explains $41.8 \%$ of the variance in accuracy $\left(\mathbb{R}=0.65, \mathbb{R}^{2}=0.42\right)$. To ensure the validity of the model, we check for the existence of multicollinearity. Our predictors report a variance inflation factor between 1.05 and 1.46, well below the often-used threshold of 5 to detect multicollinearity [25].

\begin{tabular}{lll}
\hline Predictor & Coefficient & P-value \\
\hline Task objectivity (objective) & 1.92 & $<0.001$ \\
Group size difference & 0.69 & $<0.001$ \\
Initial confidence & -0.04 & $<0.001$ \\
No discussion : private & -1.15 & 0.017 \\
No discussion : public & -1.13 & 0.017 \\
Discussion : private & -0.97 & 0.039 \\
\hline
\end{tabular}

Table 1. Effect of statistically significant predictors on participant conformity. The sign of the coefficient (+/-) denotes the direction of the relationship between the predictor and conformity behaviour. Absolute value of the coefficient determines the effect size.

We observe statistically significant main effects of task objectivity (see Fig. 5), group size difference (see Fig. 6), and initial confidence of participants (see Fig. 7), while the level of interactivity (discussion vs. no discussion) and response visibility (public vs. private) demonstrate a statistically significant interaction effect on conformity behaviour (see Fig. 8). No effect was observed for participant gender or user representations used in this experiment. Next, we present a more detailed look at the significant features.

\subsection{Task Objectivity, Group Size Difference and Initial Confidence}

Our results show that the task objectivity (objective or subjective nature of the task) had the strongest effect on conformity behaviour. Out of the 152 conformity responses, 120 responses were related to objective questions (79\%) while only 32 responses were related to subjective questions (21\%), suggesting that participants were more inclined to conform to the majority's answer in objective questions than in subjective questions. We illustrate the likelihood of participants conforming to the majority's answers in subjective and objective questions in Fig. 5. We note that in subjective questions, participant conformity ranged between $0-50 \%$ with a median value of $0 \%$, while in objective questions the value ranged between $0-100 \%$ with a higher median value of $50 \%$.

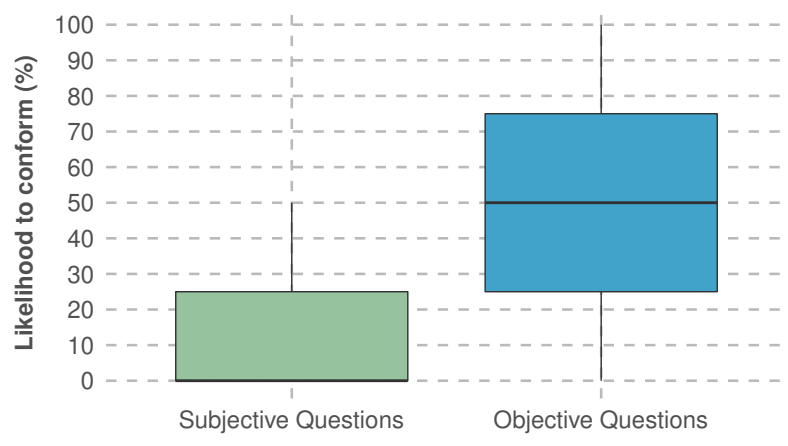

Fig. 5. The likelihood of participants conforming to the majority in subjective and objective questions. 
Moreover, the group size difference between the majority and the minority displayed a statistically significant main effect on conformity behaviour. We illustrate this relationship in Fig. 6 as a density plot, which shows the distribution of participants' likelihood of conforming, when the group size difference between the majority and the minority was 1 (majority of 3 vs. minority of 2) and 3 (majority of $4 \mathrm{vs}$. minority of 1). We note that the curve representing conformity behaviour when the group size difference is at 1 , is left-skewed (with a mean of $24 \%$ and a median of $25 \%$ ), whereas the curve representing conformity behaviour when the group size difference is at 3, is right-skewed (with a mean of $35 \%$ and a median of $25 \%$ ), suggesting that participants are more likely to conform to the majority when the difference between the groups is higher.

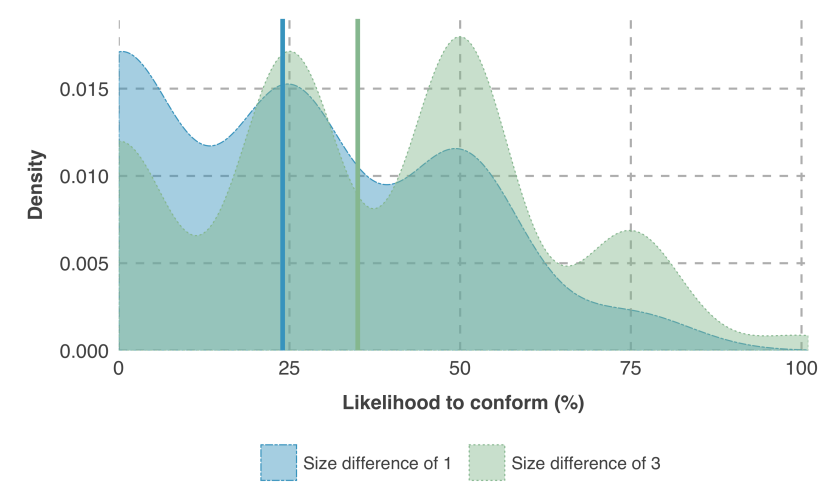

Fig. 6. The distribution of the likelihood of participants conforming to the majority against the considered group size differences. The solid vertical lines denote the mean likelihood to conform in each case.

Furthermore, our results show that individuals who display higher confidence on their initial answers are less likely to be influenced by the majority as demonstrated by the distribution of initial confidence levels of participants across non-conforming and conforming responses in Fig. 7. While the initial confidence level of participants ranges between $0-100$ in both non-conforming and conforming responses, the median initial confidence values is at 80 and 55 respectively.

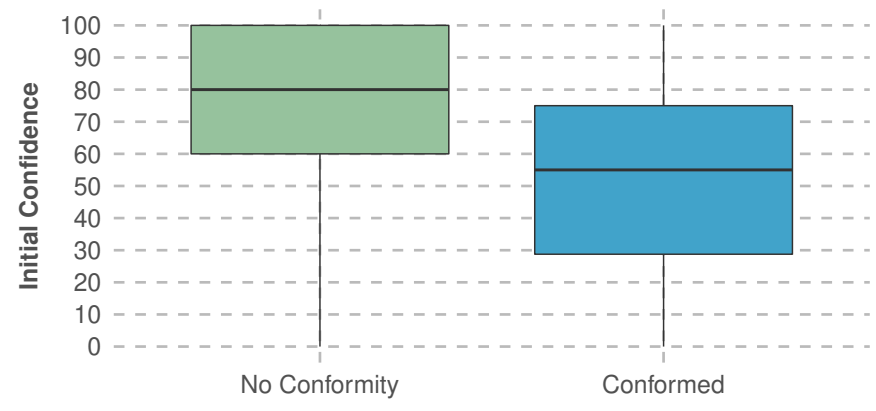

Fig. 7. Distribution of initial confidence values of participants across non-conforming and conforming responses.

\subsection{Social Presence}

We manipulated the social presence facilitated by the platform using three variables: user representation, interactivity and response visibility. While we do not find statistically significant main effects for each aspect, we note a statistically significant interaction between the level of interactivity (discussion vs. no discussion) and response visibility (public vs. private). The interaction effect between interactivity and response visibility results in four levels of social presence: discussion:public, discussion:private, no discussion:public, and no discussion:private. The effect of these four levels of social presence on conformity behaviour is illustrated in Fig. 8. 


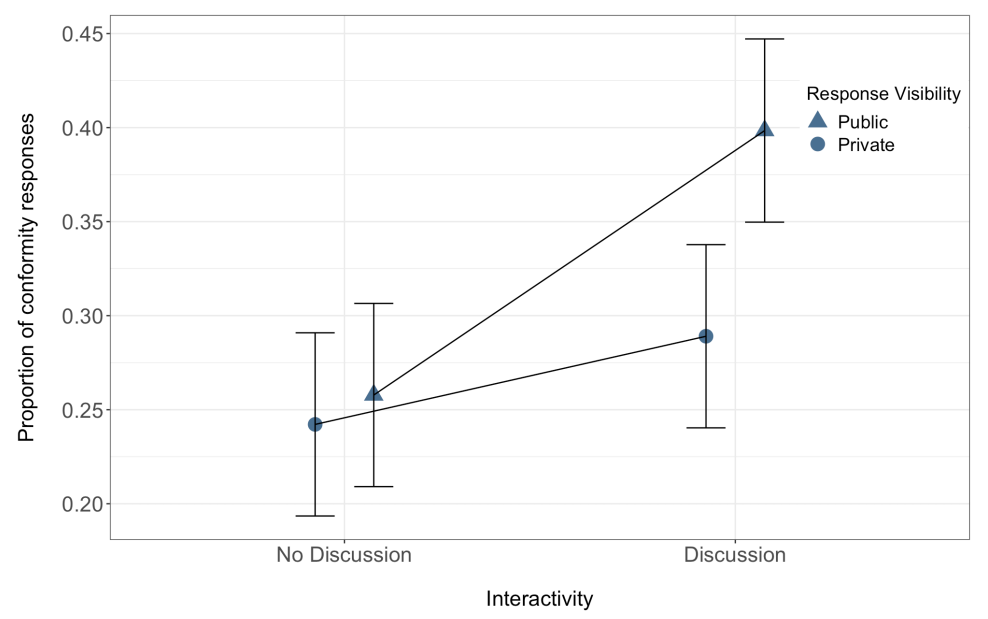

Fig. 8. Proportion of conformity responses across the four levels of interactivity and response visibility.

We highlight that highest conformity is observed when participants are provided the opportunity to discuss answers with peers, while also displaying their final answers to the rest of the group before moving to the next question (discussion:public condition). In contrast, participants are least likely to conform when there is no peer discussion, and the final responses are private. Moreover, based on our results in Table 1, we observe that even with private responses, having peer discussion is more likely to result in conformity when compared to conditions with no discussion, but with public responses.

\subsection{Qualitative Analysis}

Based on the transcripts from our semi-structured interviews, we aim to obtain a richer understanding of the quantitative results presented in the previous section. Two of the paper's authors individually transcribed and categorised the interview data, the outcomes of which were subsequently combined in an online spreadsheet to aid in the discussion and comparison of the categorisations. The two authors then collaboratively followed a deductive thematic analysis of the participants' responses [9]. In particular, our semi-structured interview aimed to identify the factors which participants believed to affect their shift in answer choices and confidence. We group our qualitative analysis across the manipulations concerning online social presence (user representation, interactivity, and response visibility) and factors previously highlighted in the (offline conformity) literature (task objectivity, majority group size, and self confidence).

4.4.1 Online Social Presence and Conformity. We altered the user representation of our participants (and their peers) using either generic avatars or user-specific avatars (using initials) and subsequently asked our participants how they perceived their assigned representation condition in contrast to the alternative. A number of participants mentioned that the use of initials created a more 'human-like' experience; "I like the initials better. It is more human and it kind of acknowledges each of us differently. We all have our own uniqueness, when compared to the generic avatars." (P17). Some participants highlighted how they subconsciously viewed user-specific avatars as 'real' people facing circumstances similar to them, which resulted in a connection to these participants; "I think in the discussion, when I saw avatars with initials, it put a person behind it for me rather than seeing "User 1" or "User 2". To me that would seem a little more automated than having avatars with initials. I think in a glimpse, I connected more with others and sometimes felt like I could relate to a particular user." (P64). Also, participants described how the use of initials supported in recognition of peers' (perceived) abilities to answer correctly; "If you have 20 - 30 questions, then we might see who is giving more correct answers consistently, with the avatars with initials." (P20). 
However, a widely shared perception among participants is that the level of representation is limited in both conditions. As described by P01, an individual's full name would reveal additional information as opposed to solely using initials; "If I see the full name and not just the initials, we can kind of guess the background of that person and where they are from. From there, you can guess what their experience and opinions might be.". Participants also describe how additional information not included in our study may be used to judge peers; "Neither option shows you their age, or experience and there is no way to know that person's background." (P32). In addition to the aforementioned factors of background and age, one participant suggested that the inclusion of academic titles would sway their judgement; "But if they have a title like "Professor", I will be more convinced." (P16).

We also asked our participants on the perceived effect of interactivity with their peers. We describe three distinct behaviours as observed in the interviews. First, participants note that chat-based interaction allows them to establish a level of confidence in the ability of their peers; "If I get a chance to talk with others, I will be more confident. I can determine whether they have the knowledge required. For example, if someone tells me 'I have been there before' to the capital of Bulgaria question, I will trust them. Else I will chose the majority." (P16). Second, participants note that the discussion allows them to obtain insights into the reasoning behind their peers' answers. This was particularly mentioned in relation to subjective questions. For example, "For most of the questions I was very firm about my answers. [...] But for subjective questions, I am happy to know why they chose their answers and then maybe change mine accordingly." (P01). Third, a small number of participants note that a group of participants could reach new insights through discussion; "Discussion will help people get new ideas as well." (P06).

However, we also note that a substantial portion of participants expressed that the discussions did not change their opinion in subjective questions. They emphasised how they have already made up their mind, and could only be swayed on objective questions if novel factual information would be shared; "I don't think that a discussion would make a change in subjective questions. I feel that I am entitled to have my opinion and they are entitled to theirs. Ijustified it pretty well in my head. For objective questions however, if I got new information from the discussion that would have made a difference." (P25).

When considering the effect of response visibility, a large portion of participants from the 'public' condition expressed concerns about the perception of their peers; "I don't want others to find me foolish. [...] I knew I was more likely to be wrong and I did not want myself to stand out and feel stupid." (P12). Participants which reported to be more at ease with the public visibility of their responses frequently cited the fact that the study was anonymous - thereby reducing the potential feeling of embarrassment; "I won't care about what others think whether I change or not. It is still anonymous, I don't think it has an effect on me." (P16). Participants did not only consider whether their peers would label them to be 'correct' or 'incorrect', but additionally considered how they were perceived. As illustrated by P46, "I might be a little pressured if they can see the changes. I would feel like they would think 'I was not good enough persuading User 3' so that adds a little pressure, because I am considering others' feelings. I don't want them to feel bad that they could not persuade me". Similarly, a participant assigned to the 'private' condition noted that even if he would not change his answer, he would use alternative signals to communicate with his peers (i.e., answer confidence); "If the amended answers were shown to others, I may reduce my confidence. Because others have different opinions and secondly, I would think others think 'he is very stubborn, he won't change his answer'. I think this would negatively affect my personality." (P14).

4.4.2 Traditional Factors and Conformity. Task Objectivity (either objective or subjective in nature) had a significant effect on participants' conformity behaviour, with participants more likely to conform on objective questions. This sentiment was also observed in our interview data, with the majority of participants describing their reluctance to switch on subjective questions. The fact that they could not be 'wrong' on subjective questions was a widespread belief among participants; "Because it is all about my feelings. There is no correct or wrong answer." (P24). Furthermore, participants note how the distinction between peer and self-expertise is more critical when considering objective 
questions; "If it was about opinions, usually I won't change because I have my own personal values about things. If it was more about intelligence, I will listen to others because I am not an expert in this area." (P16).

A number of participants reported that a more in-depth conversation would have the possibility to sway their mind on subjective questions, whereas an opposing majority on objective questions was sufficient motivation for switching to the majority opinion; "For subjective questions you need to read more about it. But for objective questions you know there is a correct answer and someone else can know it. While I wanted to know more about why others were thinking what they were thinking, two or three lines are not enough to change my opinion." (P42).

Group size differences, the size of the majority in comparison to that of the minority, were reported as having a considerable impact on participant responses. Participants were more confident in accepting answers from larger majorities than smaller majorities; "I often followed the majority because the majority may have the right answer. As we were a group of five, I was more sure of following the four person majority than a three person majority." (P18). If confronted with a unanimous majority of opposing answers, participants report conforming to the majority despite being confident in their own answer; "Even when I was pretty sure, there were some times where I changed my answer. It was because everyone else in the group chose something else." (P07).

Lastly, participants' initial confidence in their answer showed to be a significant predictor of their subsequent conforming behaviour. Our interview data confirms that participants were aware of this behaviour; "IfI had a lower confidence in the answer, I was more likely to change" (P10). Participants were unable to see the confidence of their 'peers', but this did not deter them from changing their answer; "Yes - Mainly when it was objective questions and in the case I had no idea of the answer, I often followed the majority. [...] Even if I did not know their level of confidence I still followed the majority." (P18).

\section{DISCUSSION}

As people continue to utilise online platforms to pursue social connections and support $[4,31,48$, 56, 57], their experiences are susceptible to social conformity influences as observed in physical settings $[6,43,44,56]$. Interestingly, the literature suggests that online conformity has both positive and negative implications. For instance, conformity is not desired when individuals accept incorrect group judgements over personal decisions [6,29]. Biased social information leads to frequent errors, which could even nullify the perceived benefits of collective intelligence. However, existing work also highlights the importance of social conformity in strengthening online group relationships and creating a sense of belonging [56,57]. Moreover, conformity is also seen as a means to encourage acceptable group norms and standards within online communities, improving the quality of their output [60]. Thus, it is imperative to understand what determines conformity behaviour in online settings in order to understand how future platforms can be designed to benefit from social conformity influences.

Our results show that previously established determinants of offline social conformity such as task objectivity, group size difference, and self-confidence pose significant effects on online conformity as well. We observe higher conformity in objective questions than in subjective questions, which contradicts the face-to-face literature on this regard [7]. Participants explained that conforming to the supposedly 'correct' answers of the majority in objective questions was more appealing than conforming to the majority's opinions in subjective questions to be 'liked' with a group. This implies that they are more susceptible to informational influences (the need to be 'right') than normative influences (the need to be 'liked') in online settings, possibly due to the anonymity and reduced social presence in online groups [16]. Furthermore, participants were more likely to conform as the distance between the majority and the minority increased, confirming the existing literature on majority group size in both offline and online contexts [3, 20, 30, 42, 54, 65, 67, 68]. Participants explained that larger distances between themselves and the majority exerted a sense of isolation and pressure to fit in, suggesting the existence of normative influences [16]. Moreover, we also note that participants were less pressured to conform when they were more confident about their initial responses, extending previous findings of face-to-face conformity literature $[11,61]$ to online settings. 


\subsection{Impact of Social Presence on Online Social Conformity}

Social presence is an important aspect of online interactions as it determines the degree to which we feel connected with our online correspondents $[58,62]$. While recent studies signify the importance of social presence on online user behaviour [18, 26-28, 64], its effect on conformity is underexplored. Moreover, despite being recognised as a multifaceted concept, existing work does not investigate multiple aspects of social presence simultaneously. Thus, in this study we investigate how social presence impacts online conformity behaviour across different user representations (generic vs. user-specific avatars), levels of interactivity (discussion vs. no discussion), and response visibility (public vs. private responses).

Our results show an overall conformity rate of $30 \%$, slightly lower than the conformity rates reported in face-to-face literature (usually above 33\% [2,3]). Differences in perceived social presence in face-to-face and online settings may have been a contributing factor in this regard, as highlighted by our participants during the interviews. In addition, our results imply that online social presence itself has a clear effect on conformity behaviour. While no main effects were observed for the three variables of social presence, higher social presence manipulated through interactivity (peer discussions) and response visibility (public responses) resulted in higher conformity. Our qualitative results confirm that participants in discussion conditions with public responses felt compelled to conform to the group answers, as their final answers were visible to peers subsequent to the discussion. Moreover, they were also concerned with how others would perceive their non-conforming behaviour despite the group's effort to convince them, suggesting susceptibility to normative influences. Thus, in comparison to recent work in online conformity highlighting the predominant effects of informational influences $[67,68]$, our study suggests the presence of both normative and informational influences, likely due to the added social presence via discussion and public responses.

Furthermore, we note that peer discussion itself without public responses (i.e., discussion:private) yielded more conformity than public responses in the absence of discussion (i.e., no discussion:public), suggesting that the level of interactivity imposed a higher contribution towards the perceived social presence than response visibility. Participants reported that discussion (high interactivity) provided them with an opportunity to understand the reasoning behind peer decisions, potentially increasing their confidence in the peer answers, resulting in increased conformity. Participants also highlighted how the discussion was most convincing when they could compare and relate their experiences with peer arguments, demonstrating high levels of social presence [41, 62].

We further note that the chosen user representations (generic vs. user-specific avatars) did not significantly differ in their influence on conformity. While, some participants highlighted during interviews that user-specific avatars were more effective in indicating the presence of a human user in comparison to generic avatars, the absence of explicit user cues (such as name, gender, age, etc.) may have invalidated this difference. Participants further suggested that real photographs or full names of their peers would have been more influential alternatives, as they provide more information about their peers and their background, which could have impacted their final decision.

\subsection{Implications for the Design of Online Group Settings}

Social presence is a crucial element of online platform design as it is seen to contribute towards platform attractiveness [26], user involvement and interaction [18, 26], user satisfaction [24, 52], and trust $[27,28]$. Moreover, findings from our study acknowledge that social presence may also play a vital role in online conformity behaviour. We note that social presence could manifest in other means in online settings (e.g., communication style and strategy [62]), a topic beyond the scope of this paper. Thus, we present the following implications based on how online social presence can be manipulated in terms of interactivity, response visibility, and user representation.

We note that perceived social presence can be controlled via the level of interactivity and response visibility provided by an online setting, such that enhancing interactivity and response visibility leads to higher social presence and vice versa. Higher social presence would also increase the susceptibility

Proc. ACM Hum.-Comput. Interact., Vol. 4, No. CSCW1, Article 55. Publication date: May 2020. 
of users to social influences such as conformity. Therefore, when higher conformity is desired (e.g., to encourage group norms and standards in online communities [56,60]), increasing the level of interactivity and visibility of user input is recommended. This would be particularly effective when normative conformity is desired (e.g., online support groups [56]).

While increasing perceived social presence in an online setting may seem desirable in most situations, we cannot disregard its effect on overall conformity behaviour. For example, the effect of social presence in online learning platforms has been debated in existing literature. While some studies suggest that social presence (in terms of peer interactions and feedback) leads to higher student satisfaction in online learning environments [23,24,52], other work shows that students who interact with peers are more likely to conform to erroneous judgements of their peers [6]. Therefore, platform designers should be aware of this conundrum when determining an appropriate balance between social presence and social influence.

Furthermore, our findings suggest that the visibility of user actions to others in an online setting is an important determinant of social presence as well as their conformity behaviour. When participants were told that their final answers would be visible to their peers, they were more inclined to adjust their answers in favour of the majority, as they were more concerned with being 'liked' than being 'right'. Thus, response visibility may be particularly important when it is desired to enhance normative conformity. This may be applicable to online support groups where co-dependency and group togetherness is more important compared to other online settings [56, 57].

While our results do not demonstrate a clear difference in the impact on conformity between the generic and user-specific avatars, we highlight that participants preferred user representations with more information and human-like features, as suggested by [22, 37, 47]. However, we emphasise that while more information may improve the perceived social connection between users, having richer user representations (such as photographs or anthropomorphic avatars) may also generate stereotypical behaviour as seen in prior literature [35, 36, 50, 67]. For example, in [67] participants stereotypically perceived competency of others based on gender of their avatars, which in turn influenced their conformity behaviour. Thus, we recommend that future work investigate alternative online user representations devoid of explicit user cues (e.g., name, gender, age), such as default site-specific avatars as used in Slack and Snapchat, or animal avatars as used in Google Docs as shown in Fig. 9, to facilitate social presence without triggering similar stereotypical behaviour.
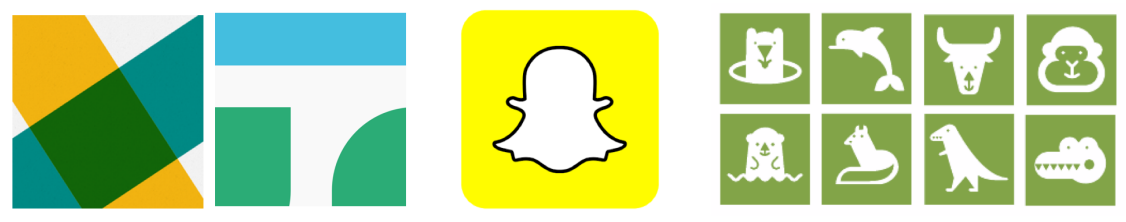

Fig. 9. Avatars used in Slack, Snapchat and Google Docs

In conclusion, our results acknowledge that social presence is a critical factor to be considered in online platform design. We emphasise that designers should pay special attention to how the level of interactivity, response visibility, and user representation could be used to control the perceived online social presence, thus also manipulating how susceptible users are to conformity influences. Future work could extend this work to investigate whether and how different platform designs could be developed, manipulating proven determinants of online social conformity (such as social presence, task objectivity, group size, self-confidence etc.) to encourage or discourage conformity and other social influences as required, to capitalise on their potential positive and negative implications. 


\subsection{Limitations}

We note the following limitations in our study. While our participants came from different educational backgrounds and levels, they demonstrated above average computer literacy. Thus, further work may be required to ensure whether these observations can be generalised to a wider population.

While within the community considered for this study (Australia), the chosen topics were acceptable as general knowledge questions (as supported by prior literature [67, 68], and our quantitative and qualitative results), they may not generate similar results cross-culturally. Different cultural backgrounds may in fact restrict the applicability of the current findings. Therefore, we encourage future research to extend our work by investigating different communities following a similar pilot test (as explained in Section 3.5) to ensure that chosen topics are acceptable as general knowledge in the targeted community.

Moreover, for the purpose of this experiment we defined social presence in terms of three aspects: user representation, interactivity, and response visibility. However, we note that social presence is a broader concept with other dimensions (e.g., social context, online communication [62]), which we did not consider in this study to avoid overly complicating the experimental setup. In addition, the aspects we did consider can also manifest in other ways. For example, interactivity can be determined via the communication style and formality of language in addition to what was tested in this study [62]. Therefore, we note that our study provides an initial step for future work that could further investigate other dimensions and aspects of social presence in online environments.

\section{CONCLUSION}

Recent literature on conformity has given a significant emphasis to its diverse manifestations in online settings [6, 43, 44, 56, 60, 67-69]. However, limited effort has been invested in understanding what triggers conformity in online settings. Therefore, this study investigated the effect of social presence (i.e., the awareness of and the sense of being connected to others via mediated communication [58]) on online conformity, while also accounting for the effects of majority group size, task objectivity, and self-confidence - well-known determinants of offline conformity. We manipulated the social presence facilitated by our study setup across three variables - user representation, interactivity, and response visibility - to investigate their combined effect on social conformity.

Our results reveal that in addition to the expected effects from group size difference, task objectivity, and self-confidence, the interaction among certain aspects of social presence also play a vital role on online conformity behaviour. We note that higher levels of online social presence (in terms of interactivity and response visibility) heightens the susceptibility of users to normative influences in addition to the commonly observed informational influences, leading to higher conformity in online settings.

We conclude with a discussion on what our findings imply for the design of online group settings. First, we emphasise that social presence can indeed be used to control conformity influences in online settings. Second, our results suggest that platform designers can manipulate the level of interactivity facilitated and the visibility of user actions to manage online social presence, which in turn could help regulate conformity influences. Thus, designers should pay special attention to the above aspects of social presence in order to encourage and shape user behaviour as desired in a given setting. We also highlight that the 'correct' level of social presence varies based on the requirement of the platform and thus should be determined after careful consideration of its pros and cons. Moreover, our findings emphasise that online users prefer user representations that can provide more user-specific information. However, as a substantial amount of literature investigating online user representations and stereotyping recommends otherwise [35, 36, 67], we suggest alternatives that provide a compromise between stronger user cues and stereotypical conformity. We encourage future work to investigate effects of other dimensions and variables of social presence, such as social context and communication style [62], on online conformity that can expand upon these implications. 


\section{REFERENCES}

[1] John G Adair. 1972. Demand characteristics or conformity?: Suspiciousness of deception and experimenter bias in conformity research. Canadian fournal of Behavioural Science/Revue canadienne des sciences du comportement 4, 3 (1972), 238.

[2] Solomon E Asch. 1951. Groups, leadership and men. Carnegie Press, Oxford, England, Chapter: Effects of group pressure upon the modification and distortion of judgements, 177-190.

[3] Solomon E Asch. 1955. Opinions and social pressure. Scientific American 193, 5 (1955), 31-35.

[4] Louise Barkhuus and Juliana Tashiro. 2010. Student Socialization in the Age of Facebook. In Proceedings of the SIGCHI Conference on Human Factors in Computing Systems (CHI '10). ACM, New York, NY, USA, 133-142. https://doi.org/10.1145/1753326.1753347

[5] Douglas Bates, Martin Mächler, Ben Bolker, and Steve Walker. 2015. Fitting Linear Mixed-Effects Models Using lme4. fournal of Statistical Software 67, 1 (2015), 1-48.

[6] Tanya Beran, Michelle Drefs, Alyshah Kaba, Noof Al Baz, and Nouf Al Harbi. 2015. Conformity of responses among graduate students in an online environment. The Internet and Higher Education 25 (2015), 63-69.

[7] Robert R Blake, Harry Helson, and Jane Srygley Mouton. 1957. The Generality of Conformity Behavior as a Function of Factual Anchorage. Difficulty of Task, and Amount of Social Pressure. Journal of Personality 25, 3 (1957), $294-305$.

[8] Benjamin M. Bolker, Mollie E. Brooks, Connie J. Clark, Shane W. Geange, John R. Poulsen, M. Henry H. Stevens, and Jada-Simone S. White. 2009. Generalized linear mixed models: a practical guide for ecology and evolution. Trends in Ecology \& Evolution 24, 3 (2009), 127-135.

[9] Virginia Braun and Victoria Clarke. 2006. Using thematic analysis in psychology. Qualitative Research in Psychology 3, 2 (2006), 77-101. https://doi.org/10.1191/1478088706qp063oa

[10] Michael J. Brzozowski, Phil Adams, and Ed H. Chi. 2015. Google+ Communities As Plazas and Topic Boards. In Proceedings of the 33rd Annual ACM Conference on Human Factors in Computing Systems (CHI '15). ACM, New York, NY, USA, 3779-3788. https://doi.org/10.1145/2702123.2702600

[11] Jennifer D Campbell, Abraham Tesser, and Patricia J Fairey. 1986. Conformity and attention to the stimulus: Some temporal and contextual dynamics. Fournal of Personality and Social Psychology 51, 2 (1986), 315-324.

[12] Emily Christofides, Towhidul Islam, and Serge Desmarais. 2009. Gender stereotyping over instant messenger: The effects of gender and context. Computers in Human Behavior 25, 4 (2009), 897-901.

[13] Robert B Cialdini. 2001. Harnessing the science of persuasion. Harvard Business Review 79, 9 (2001), 72-81.

[14] Robert B Cialdini and Noah J Goldstein. 2004. Social influence: Compliance and conformity. Annu. Rev. Psychol. 55 (2004), 591-621.

[15] Joanie B Connell, Gerald A Mendelsohn, Richard W Robins, John Canny, and John Canny. 2001. Effects of communication medium on interpersonal perceptions. In Proceedings of the 2001 International ACM SIGGROUP Conference on Supporting Group Work. ACM, 117-124.

[16] Morton Deutsch and Harold B Gerard. 1955. A study of normative and informational social influences upon individual judgment. The fournal of Abnormal and Social Psychology 51, 3 (1955), 629-636.

[17] Leonard W Ferguson. 1944. An analysis of the generality of suggestibility to group opinion. Fournal of Personality 12, 3 (1944), 237-243.

[18] David R Fortin and Ruby Roy Dholakia. 2005. Interactivity and vividness effects on social presence and involvement with a web-based advertisement. Journal of business research 58, 3 (2005), 387-396.

[19] D Randy Garrison, Terry Anderson, and Walter Archer. 2001. Critical thinking, cognitive presence, and computer conferencing in distance education. American fournal of distance education 15, 1 (2001), 7-23.

[20] Solomon C Goldberg. 1954. Three situational determinants of conformity to social norms. The fournal of Abnormal and Social Psychology 49, 3 (1954), 325-329.

[21] Jorge Goncalves, Vassilis Kostakos, and Jayant Venkatanathan. 2013. Narrowcasting in Social Media: Effects and Perceptions. In Proceedings of the 2013 IEEE/ACM International Conference on Advances in Social Networks Analysis and Mining (ASONAM '13). ACM, New York, NY, USA, 502-509.

[22] Li Gong. 2008. How social is social responses to computers? The function of the degree of anthropomorphism in computer representations. Computers in Human Behavior 24, 4 (2008), 1494-1509.

[23] Charlotte N Gunawardena. 1995. Social presence theory and implications for interaction and collaborative learning in computer conferences. International journal of educational telecommunications 1, 2 (1995), 147-166.

[24] Charlotte N Gunawardena and Frank J Zittle. 1997. Social presence as a predictor of satisfaction within a computer-mediated conferencing environment. American journal of distance education 11, 3 (1997), 8-26.

[25] Joseph F Hair, William C Black, Barry J Babin, Rolph E Anderson, and RL Tatham. 2010. Multivariate Data Analysis. Pearson, New Jersey, NJ, USA.

[26] Khaled Hassanein and Milena Head. 2007. Manipulating perceived social presence through the web interface and its impact on attitude towards online shopping. International fournal of Human-Computer Studies 65, 8 (2007), 689-708.

[27] Khaled S Hassanein and M Head. 2004. Building online trust through socially rich web interfaces. In Proceedings of the 2 nd annual conference on privacy, security and trust, Fredericton, New Brunswick, Canada. 15-22.

[28] Traci J Hess, Mark Fuller, and Damon E Campbell. 2009. Designing interfaces with social presence: Using vividness and extraversion to create social recommendation agents. Journal of the Association for Information Systems 10, 12 (2009), 1.

Proc. ACM Hum.-Comput. Interact., Vol. 4, No. CSCW1, Article 55. Publication date: May 2020. 
[29] Jessica Hullman, Eytan Adar, and Priti Shah. 2011. The Impact of Social Information on Visual Judgments. In Proceedings of the SIGCHI Conference on Human Factors in Computing Systems (CHI '11). ACM, New York, NY, USA, 1461-1470. https://doi.org/10.1145/1978942.1979157

[30] Chester A. Insko, Richard H. Smith, Mark D. Alicke, Joel Wade, and Sylvester Taylor. 1985. Conformity and Group Size: The Concern with Being Right and the Concern with Being Liked. Personality and Social Psychology Bulletin 11, 1(1985), 41-50.

[31] Adam N. Joinson. 2008. Looking at, Looking Up or Keeping Up with People?: Motives and Use of Facebook. In Proceedings of the SIGCHI Conference on Human Factors in Computing Systems (CHI '08). ACM, New York, NY, USA, 1027-1036. https://doi.org/10.1145/1357054.1357213

[32] Jitendra Kumar. 1983. Conformity behavior as a function of confederates' age and size of the confederate group. Personality Study \& Group Behaviour 3 (1983), 69-73.

[33] Lieve Laporte, Christof van Nimwegen, and Alex J. Uyttendaele. 2010. Do People Say What They Think: Social Conformity Behavior in Varying Degrees of Online Social Presence. In Proceedings of the 6th Nordic Conference on Human-Computer Interaction: Extending Boundaries (NordiCHI '10). ACM, New York, NY, USA, 305-314.

[34] Bibb Latané. 1981. The psychology of social impact. American psychologist 36, 4 (1981), 343.

[35] Eun-Ju Lee. 2004. Effects of gendered character representation on person perception and informational social influence in computer-mediated communication. Computers in Human Behavior 20, 6 (2004), 779-799.

[36] Eun-Ju Lee. 2007. Wired for gender: Experientiality and gender-stereotyping in computer-mediated communication. Media Psychology 10, 2 (2007), 182-210.

[37] Eun-Ju Lee and Clifford Nass. 2002. Experimental tests of normative group influence and representation effects in computer-mediated communication: When interacting via computers differs from interacting with computers. Human Communication Research 28, 3 (2002), 349-381.

[38] John M Levine. 1999. Solomon Asch's legacy for group research. Personality and Social Psychology Review 3, 4 (1999), 358-364.

[39] Wendy Liu and Derek Ruths. 2013. What's in a name? Using first names as features for gender inference in twitter, In 2013 AAAI Spring Symposium Series. AAAI Spring Symposium - Technical Report SS-13-01, 10-16.

[40] Yong Liu, Jayant Venkatanathan, Jorge Goncalves, Evangelos Karapanos, and Vassilis Kostakos. 2014. Modeling What Friendship Patterns on Facebook Reveal About Personality and Social Capital. ACM Trans. Comput.-Hum. Interact. 21, 3, Article Article 17 (June 2014), 20 pages. https://doi.org/10.1145/2617572

[41] Patrick R Lowenthal and Chareen Snelson. 2017. In search of a better understanding of social presence: an investigation into how researchers define social presence. Distance Education 38, 2 (2017), 141-159.

[42] Paul Benjamin Lowry, Tom L Roberts, Nicholas C Romano Jr, Paul D Cheney, and Ross T Hightower. 2006. The impact of group size and social presence on small-group communication: Does computer-mediated communication make a difference? Small Group Research 37, 6 (2006), 631-661.

[43] Misa Maruyama, Scott P. Robertson, Sara Douglas, Roxanne Raine, and Bryan Semaan. 2017. Social Watching a Civic Broadcast: Understanding the Effects of Positive Feedback and Other Users' Opinions. In Proceedings of the 2017 ACM Conference on Computer Supported Cooperative Work and Social Computing (CSCW '17). ACM, New York, NY, USA, 794-807.

[44] Misa T. Maruyama, Scott P. Robertson, Sara K. Douglas, Bryan C. Semaan, and Heather A. Faucett. 2014. Hybrid Media Consumption: How Tweeting During a Televised Political Debate Influences the Vote Decision. In Proceedings of the 17th ACM Conference on Computer Supported Cooperative Work \& Social Computing (CSCW'14). ACM, New York, NY, USA, 1422-1432.

[45] Katelyn YA McKenna and Amie S Green. 2002. Virtual group dynamics. Group Dynamics: Theory, Research, and Practice 6, 1 (2002), 116-127.

[46] Brian Mullen. 1983. Operationalizing the effect of the group on the individual: A self-attention perspective. Fournal of Experimental Social Psychology 19, 4 (1983), 295-322.

[47] Kristine L Nowak and Frank Biocca. 2003. The effect of the agency and anthropomorphism on users' sense of telepresence, copresence, and social presence in virtual environments. Presence: Teleoperators \& Virtual Environments 12, 5 (2003), 481-494.

[48] Julie Prescott, Terry Hanley, and Katalin Ujhelyi Gomez. 2019. Why do young people use online forums for mental health and emotional support? Benefits and challenges. British fournal of Guidance \& Counselling (2019), 1-11.

[49] Vincent Price, Lilach Nir, and Joseph N Cappella. 2006. Normative and informational influences in online political discussions. Communication Theory 16, 1 (2006), 47-74.

[50] Rabindra Ratan, David Beyea, Benjamin J Li, and Luis Graciano. 2019. Avatar characteristics induce users' behavioral conformity with small-to-medium effect sizes: a meta-analysis of the proteus effect. Media Psychology (2019), 1-25.

[51] Bernardo Reynolds, Jayant Venkatanathan, Jorge Gonçalves, and Vassilis Kostakos. 2011. Sharing Ephemeral Information in Online Social Networks: Privacy Perceptions and Behaviours. In Human-Computer Interaction - INTERACT 2011, Pedro Campos, Nicholas Graham, Joaquim Jorge, Nuno Nunes, Philippe Palanque, and Marco Winckler (Eds.). Springer Berlin Heidelberg, Berlin, Heidelberg, 204-215.

[52] Jennifer Richardson and Karen Swan. 2003. Examing social presence in online courses in relation to students' perceived learning and satisfaction. Journal of Asynchronous Learning 7, 1 (2003), 68-88. 
[53] Michael Rosander and Oskar Eriksson. 2012. Conformity on the Internet-The role of task difficulty and gender differences. Computers in Human Behavior 28, 5 (2012), 1587-1595.

[54] Leon Rosenberg. 1961. Group size, prior experience, and conformity. The fournal of Abnormal and Social Psychology 63, 2 (1961), 436-437.

[55] Victor Savicki, Merle Kelley, and E Oesterreich. 1999. Judgments of gender in computer-mediated communication. Computers in Human Behavior 15, 2 (1999), 185-194.

[56] Eva Sharma and Munmun De Choudhury. 2018. Mental Health Support and Its Relationship to Linguistic Accommodation in Online Communities. In Proceedings of the 2018 CHI Conference on Human Factors in Computing Systems (CHI '18). ACM, New York, NY, USA, Article 641, 13 pages.

[57] Eva Sharma and Munmun De Choudhury. 2018. Mental Health Support and Its Relationship to Linguistic Accommodation in Online Communities. In Proceedings of the 2018 CHI Conference on Human Factors in Computing Systems (CHI '18). ACM, New York, NY, USA, Article 641, 13 pages. https://doi.org/10.1145/3173574.3174215

[58] John Short, Ederyn Williams, and Bruce Christie. 1976. The social psychology of telecommunications. John Wiley \& Sons.

[59] David J Stang. 1976. Ineffective deception in conformity research: Some causes and consequences. European fournal of Social Psychology 6, 3 (1976), 353-367.

[60] Abhay Sukumaran, Stephanie Vezich, Melanie McHugh, and Clifford Nass. 2011. Normative Influences on Thoughtful Online Participation. In Proceedings of the SIGCHI Conference on Human Factors in Computing Systems (CHI '11). ACM, New York, NY, USA, 3401-3410.

[61] Abraham Tesser, Jennifer Campbell, and Susan Mickler. 1983. The role of social pressure, attention to the stimulus, and self-doubt in conformity. European fournal of Social Psychology 13, 3 (1983), 217-233.

[62] Chih-Hsiung Tu and Marina McIsaac. 2002. The relationship of social presence and interaction in online classes. The American journal of distance education 16, 3 (2002), 131-150.

[63] Jayant Venkatanathan, Evangelos Karapanos, Vassilis Kostakos, and Jorge Gonçalves. 2012. Network, Personality and Social Capital. In Proceedings of the 4th Annual ACM Web Science Conference (WebSci '12). Association for Computing Machinery, New York, NY, USA, 326-329. https://doi.org/10.1145/2380718.2380760

[64] Jayant Venkatanathan, Vassilis Kostakos, Evangelos Karapanos, and Jorge Gonçalves. 2013. Online Disclosure of Personally Identifiable Information with Strangers: Effects of Public and Private Sharing. Interacting with Computers 26, 6 (11 2013), 614-626. https://doi.org/10.1093/iwc/iwt058 arXiv:https://academic.oup.com/iwc/articlepdf/26/6/614/1962602/iwt058.pdf

[65] Eva Walther, Herbert Bless, Fritz Strack, Patsy Rackstraw, Doris Wagner, and Lioba Werth. 2002. Conformity effects in memory as a function of group size, dissenters and uncertainty. Applied Cognitive Psychology: The Official fournal of the Society for Applied Research in Memory and Cognition 16, 7 (2002), 793-810.

[66] Senuri Wijenayake, Niels van Berkel, and Jorge Goncalves. 2020. Bots for Research: Minimising the Experimenter Effect. In International Workshop on Detection and Design for Cognitive Biases in People and Computing Systems (CHI'20 Workshop). ACM, 9 pages.

[67] Senuri Wijenayake, Niels van Berkel, Vassilis Kostakos, and Jorge Goncalves. 2019. Measuring the Effects of Gender on Online Social Conformity. Proc. ACM Hum.-Comput. Interact. 3, CSCW, Article 145 (Nov. 2019 ), 24 pages. https://doi.org/10.1145/3359247

[68] Senuri Wijenayake, Niels van Berkel, Vassilis Kostakos, and Jorge Goncalves. 2020. Impact of contextual and personal determinants on online social conformity. Computers in Human Behavior (2020), 106302.

[69] Haiyi Zhu, Bernardo Huberman, and Yarun Luon. 2012. To Switch or Not to Switch: Understanding Social Influence in Online Choices. In Proceedings of the SIGCHI Conference on Human Factors in Computing Systems (CHI '12). ACM, New York, NY, USA, 2257-2266. https://doi.org/10.1145/2207676.2208383

Received January 2020; accepted March 2020 


\section{Chapter 8}

\section{Leveraging Conformity Influences for Prosocial Behaviours}

\subsection{Introduction}

In Chapters 4, 5, $6 \&$ 7, we systematically investigate the effects of popular contextual and personal determinants of social conformity in online groups, identify their potential outcomes, and present guidelines to help control conformity influences through online platform design. The final step of our work is to consolidate the findings of these chapters to empirically determine if online conformity influences can be used to regulate user behaviour in everyday online group settings (e.g., social media).

Prior work shows that fake news spreads faster and deeper than genuine news articles in social media mainly as a result of human user behaviours and not social bots [173]. Furthermore, Colliander [32] found that mechanisms that use social information - i.e. displaying comments that are supportive or critical of an article's trustworthiness posted by other users under an article - are more influential with regards to how social media users perceive trustworthiness of online news content, in comparison to third-party disclaimers insinuating a news article might be fake. However, these effects were reported in the presence of unanimously critical or supportive comments - whereas in reality a news article posted on social media is likely to have a combination of both supportive and critical comments.

In this chapter we investigate the above findings in relation to social conformity influences. More specifically, we examine conformity in how users perceive trustworthiness of online news articles both before and after they are exposed to different combinations of comments that are either support the authenticity of the article (supportive comments), or criticise it as fake news (critical comments). We test different majority-minority compositions of supportive and critical comments, and account for user gender, age and confidence on their initial perception of an article's trustworthiness, in order to quantify the impact of previously identified conformity determinants. Furthermore, we investigate how being exposed to user comments impacts the likelihood of users reacting, commenting, sharing, fact-checking and reporting a news article on social media - which directly determines the dispersion of online news content, and hence reveal outcomes of potential conforming and non-conforming user behaviours.

Our results reveal that users readily conform to the majority's sentiment on an article's trustworthiness that they infer through comments posted by previous users, and perceive the news article as fake or real accordingly. We note that this behaviour is heightened when the majority group increases in size (i.e. when the number of comments supporting the majority's sentiment increases) as well as when the majority's sentiment is critical 
rather than supportive. Moreover, users are more susceptible to conformity when they have lower confidence on their initial perception of an article's trustworthiness. No age or gender differences in conformity are observed. Therefore, these findings provide empirical evidence for effects of conformity influences in an everyday online group setting, while also corroborating our findings in Articles I, II, III \& IV.

Furthermore, our analysis shows that those who conform to the majority's critical or supportive sentiments also adjust how they would respond to articles that they perceived as fake or real. Concisely, those who conformed to the majority's critical sentiment of an article's trustworthiness show higher inclination to take action against the dispersion of the news article by reporting it, in comparison to those who showed non-conformity. Similarly, users who conform to a majority supportive of an article's trustworthiness are significantly more likely to share, comment, and react on the news article to enable its further dispersion than those who did not conform to a supportive majority. Hence, these findings imply that social media platforms can trigger social conformity influences by displaying an appropriately filtered set of user comments to effectively communicate the authenticity of online news content and moderate how users respond to them, to not only mitigate the circulation of fake news, but also to encourage the circulation of real news articles. We describe more details of the study design and its implications in the attached publication, Article V.

\subsection{Article V}

Copyright is held by IEEE. This is the authors' version of the work. It is posted here for your personal use. Not for redistribution. The definitive Version of Record was published in:

Wijenayake, S., Hettiachchi, D., Hosio, S., Kostakos, V., Goncalves, J. (2020). Effect of Conformity on Perceived Trustworthiness of News in Social Media. IEEE Internet Computing, 25(1), 12-19. https://doi.org/10.1109/MIC.2020.3032410.

Ethics ID: 2056168, The University of Melbourne Human Ethics Advisory Group. 


\title{
Effect of Conformity on Perceived Trustworthiness of News in Social Media
}

Senuri Wijenayake

The University of Melbourne

Danula Hettiachchi

The University of Melbourne

Simo Hosio

University of Oulu

Vassilis Kostakos

The University of Melbourne

Jorge Goncalves

The University of Melbourne

\begin{abstract}
A catalyst for the spread of fake news is the existence of comments that users make in support of, or against, such articles. In this study we investigate whether critical and supportive comments can induce conformity in how readers perceive trustworthiness of news articles and respond to them. We find that individuals tend to conform to the majority's opinion of an article's trustworthiness $(58 \%)$, especially when challenged by larger majorities who are critical of the article's credibility, or when less confident about their personal judgement. Moreover, we find that individuals who conform are more inclined to take action: to report articles they perceive as fake, and to comment on and share articles they perceive as real. We conclude with a discussion on the implications of our findings for mitigating the dispersion of fake news on social media.
\end{abstract}

INTRODUCtion Social media platforms are increasingly becoming the primary source of news and information for most people. In a recent survey, $68 \%$ of Americans reported to at least occasionally consume news through social media, with Facebook being the most commonly used platform [1]. People perceive social media to be a more convenient, cheaper and timely alternative to traditional news sources, with the additional opportunity to interact with others by commenting and sharing news articles [1].

However, the convenience, cost-efficiency and accessibility offered by online social media that helped gain its fame, has also resulted in these platforms being exploited for the rapid dispersion of fake news i.e., "news articles that are intentionally and verifiably false, and could mislead readers" [2]. Purveyors of fake news attempt to sway the public's opinion to accept biased or false information to support political propaganda, personal ideology or to gain financial incentives [3]. While the Pew Research Center reports that most users tend to question the veracity of news they consume through social media [1], other reports (e.g., [2], [4]) emphasising the influence of fake news on the 2016 U.S. presidential election sug- 
gest that a majority of the general population is still largely susceptible to fake news.

As a result, determining what factors influence the perceived trustworthiness of news articles appearing on social media (i.e., whether an article is fake or real) has become a critical research topic. While existing work highlight how comments appearing on news articles influence opinion formation in subsequent users [5], [6], [7], their impact on how users perceive the trustworthiness of a news article is yet to be investigated. Furthermore, to fully comprehend the forces at play, it is vital to understand how socio-psychological concepts such as 'social conformity' may influence how people perceive trustworthiness and respond to news articles appearing on social media.

Therefore, this study intends to investigate whether and how the composition of user comments either supporting or criticising a news article posted on Facebook, may trigger conformity in how subsequent users assess its trustworthiness. Moreover, we aim to explore how users' perceived trustworthiness of a news article may influence how they respond to it in social media, which is also crucial to the dispersion of online news.

\section{Related Work}

The influence of fake news stories that disseminate through social media has been undeniable since the 2016 U.S. presidential elections. Studies report that an average American encountered between 1 to 3 fake news stories on social media in the month leading to the election [2], which they often accepted as genuine information [7]. Others also report that a majority of such fake news stories were in favour of Donald Trump, which may have been a significant determinant of his victory in the election [4].

Previous work revealed that fake news spreads faster and deeper than genuine news articles in social media, due to the behaviour of human users and not social bots [8]. Therefore, existing literature exploring computational, expert-oriented and crowdsourcing approaches to determine the veracity of social media news articles [9] may not be sufficient to mitigate the dispersion of fake news. For instance, Facebook attempted to mitigate the dispersion of fake news by displaying disclaimers on certain news articles, to alert users that the article might be fake. Contrary to expectations, displaying disclaimers disputing an article's content was seen to further establish incorrect preconceptions of users [10].

Moreover, literature recognises the impact of user comments on opinions of news readers in online contexts. Studies suggest that user comments may have a higher impact on a reader's opinion than the article itself [5]. Furthermore, in cases where user comments disagree with an article's viewpoint, readers' opinions have been seen to align with that of user comments [6].

In a recent study, Colliander [11] investigated the impact of comments posted by previous readers on a fake news article posted on Facebook, on the attitude and response of subsequent readers. The results indicate that exposure to comments critical of a fake news article adversely impacted participants' attitude on the article and lowered their likelihood to positively comment or share the article on Facebook. Alternatively, supportive comments were seen to favourably impact participants' attitude on the fake news article and increase their likelihood to comment positively and share the article. Furthermore, the above results were compared with an article consisting of supportive comments as well as a disclaimer alerting participants that the article might actually be fake, which did not lower participants' attitude or their likelihood to comment and share the post. The author concluded that comments from other users are more influential than a disclaimer from a social media platform, due to effects of 'social conformity', i.e., the human tendency to adjust personal opinions to agree with a group majority, seeking social approval (normative conformity) or presuming the majority to be 'correct' in uncertain situations (informational conformity) [12].

While Colliander's study established the influence of conformity to user comments on a Facebook news article, it only investigated conformity in the presence of unanimously critical or supportive comments, while in reality a news article could have a combination of supportive and critical comments (e.g., a majority of supportive comments vs. a minority of critical comments and vice versa). This is especially crucial as literature strongly emphasises on the effect of majority - minority group compositions on conformity behaviour [13], [14], [15]. Furthermore, 
conforming to user comments could also depend on personal determinants of users such as their self-confidence [13], [14], [15], familiarity with the news article, gender, age and time spent on social media which are yet to be investigated.

Moreover, further work is required to investigate how users respond to articles they perceive as fake or real, across a wider variety of responses that could enhance or mitigate article dispersion (i.e., their likelihood to 'react', report and factcheck the post in addition to commenting and sharing it), to fully understand the impact of conforming to others' opinions on the dispersion of news articles and whether it could effectively correct misinformation.

\section{Method}

We deployed an online survey consisting of 28 Facebook posts including 14 fake and 14 real news articles. The use of Facebook posts for the study was inspired by previous work investigating conformity in social media [11]. All fake and real news articles were extracted from Snopes - a popular fact-checking website. The articles chosen were fact-checked by Snopes between Jan 2019 - Feb 2020 based on popular demand and were labelled as either 'false' (fake news) or 'true' (real news). We did not include news articles that may directly favour a specific political affiliation to avoid possible confounding effects.

We manipulated the total number of comments appearing on the Facebook post $(2-4$; a minimum of 2 to create a 'group' opinion and a maximum of 4 as previously seen sufficient to elicit conformity by Colliander [11]) and their arrangement (in terms of the number of critical/supportive comments, and their presentation order). This meant that each post tested a unique combination of critical and supportive comments, with either a majority of critical comments, a majority of supportive comments or with an equal number of critical and supportive comments (no majority). The considered arrangements of comments were equally tested on both fake and real news articles.

All supportive and critical comments included in each post were extracted from the relevant original news article. We defined a comment posted by a user that supports the authenticity of the article as a 'supportive' comment (e.g., "Scary scenario and quite plausible!"), and any comment posted by a user questioning the authenticity of the article or directly criticising it as fake news or misinformation as a 'critical' comment (e.g., "People, please stop spreading fake news. At least some of you should be smarter than this"). We note that in the original news articles, the "most relevant" user comments were displayed based on their popularity and engagement [16]. Hence, we chose the top critical and supportive comments from each post, to be utilised in the experiment.

The survey was deployed on Amazon Mechanical Turk with the participation of 52 US residents ( 26 women and 26 men) over the age of 18 years $(M=36, S D=9.25)$. All participants were registered users of Facebook and had completed more than 1000 HITs with an approval rate above $95 \%$, a commonly used prequalification criteria used in MTurk studies [17]. Furthermore, participants were provided a downloadable Plain Language Statement with the instructions and the requirements of the survey before accepting the task. Upon accepting the task, they were asked to provide their demographic information (age, selfdisclosed gender, level of education completed and the approximate number of hours spent on social media). Subsequent to submitting their information, participants could then initiate the survey.

The survey was designed to capture the change in participants' personal opinion of an article's trustworthiness after being exposed to others' comments. At first, each post was displayed without user comments, i.e., only the cover image and the headline used in the original article were visible as shown in Figure 1 (a). Participants were instructed to imagine the post appearing on their news feed as shared by one of their distant friends on Facebook. They were then asked to rate their familiarity of the article (on a scale of $0-100,0=$ low familiarity and 100 $=$ high familiarity), their opinion on the article's trustworthiness (on a scale of $0-100,0=$ critical and $100=$ supportive) and their confidence on the provided trustworthiness rating (on a scale of $0-100,0=$ low confidence and $100=$ high confidence). Participants were then prompted to indicate whether and how they would respond to the post by reacting, commenting, fact-checking, sharing or reporting it (each on a scale of 0 - 
(a)

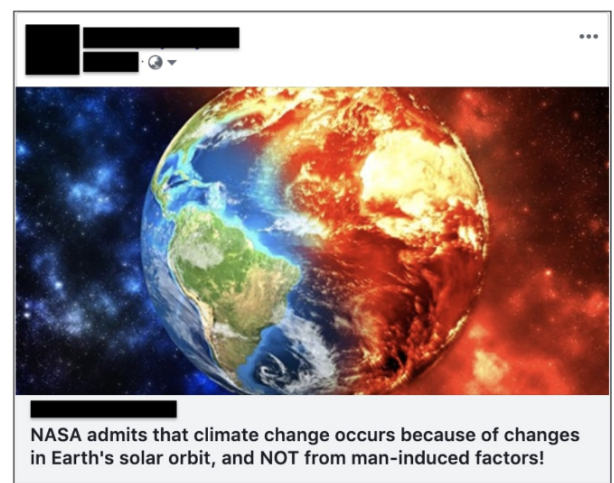

(b)

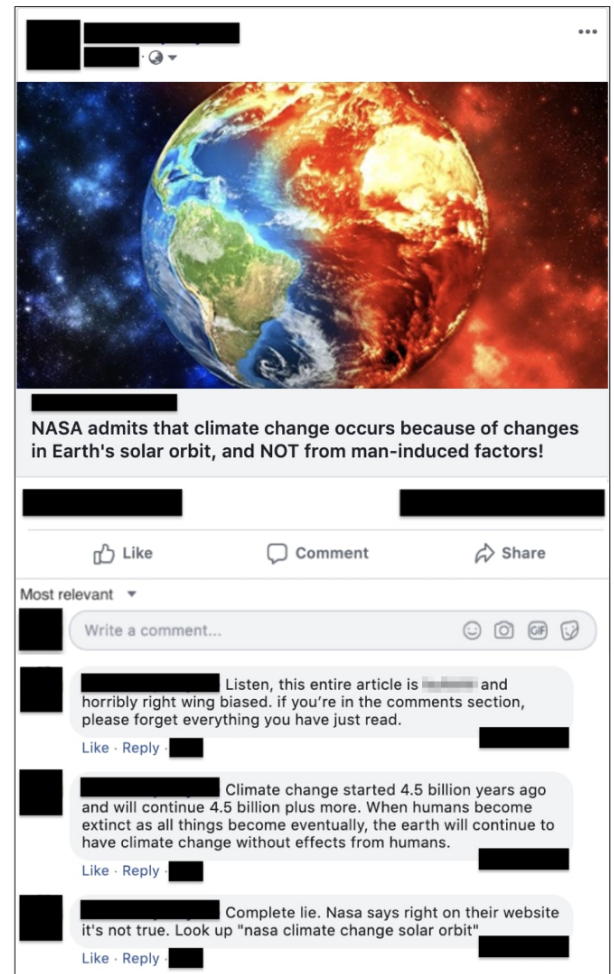

Figure 1. An example of a fake news article used in the survey, both with and without user comments.

$100,0=$ extremely unlikely and $100=$ extremely likely), based on their initial assessment of the article's trustworthiness.

Upon submitting their initial answers, we displayed the complete post with user comments as shown in Figure 1 (b). Participants were instructed to read the comments carefully and determine whether the displayed user comments are mostly supportive or critical of the post's trustworthiness. In response, participants could select one option among "supportive", "critical" or "equally distributed among the two". This question was used as a Gold Standard question to determine whether participants have read through the comments with adequate attentiveness before moving on to the next step.

After displaying the user comments and answering the Gold Standard question, participants were again requested to provide their opinion on the article's trustworthiness and their confidence on the new trustworthiness rating. Subsequently, they were also prompted to provide new ratings to reflect their likelihood to react, comment, factcheck, share or report the post after reading user comments. This approach allowed us to capture the effect of previous user comments on participants' personal opinion on the article's trustworthiness and how they subsequently chose to respond to the post. This process was repeated for each post in the survey.

The experimental design was approved by the Ethics Committee of our university. The experiment lasted for approximately $30-45$ minutes per participant. Participants who answered the survey in full, with at least $80 \%$ of correct answers for the Gold Standard questions received a payment of \$10 (USD) for participation.

\section{Results}

All 52 participants answered the 28 survey items which resulted in a total of 1456 responses, equally distributed among fake and real news articles. Of these, in 1040 responses participants faced a clear majority, either supportive or critical of the article's trustworthiness. In the remaining 416 responses, the previous user comments had an equal number of supportive and critical comments.

For the purpose of this study we define conformity as a binary variable, i.e., increasing trustworthiness rating after seeing a majority of supportive comments and reducing the trustworthiness rating after seeing a majority of critical comments were considered as conformity.

We observed conformity in 604 out of the 1040 responses where there was a clear majority (either supportive or critical), leading to a $58 \%$ conformity rate. All participants conformed at least once during the survey, with an average of 11.62 conformity responses $(S D=3.44)$ per 
participant. We then investigated the impact of the following predictor variables on the conformity behaviour of our participants, to understand factors that may have influenced their behaviour.

- Majority opinion: Supportive or critical majority.

- Majority size: Size of the majority (range: 2 $-4)$.

- Minority size: Size of the minority (values: 1 or 0$)$.

- Group size difference: Difference between the majority group size and the participant's group size (range : $0-4$ ).

- Num. of comments: Total number of previous user comments (range : 2 - 4).

- Num. of critical comments: Num. of comments critical of the article's trustworthiness (range : $0-4)$.

- Num. of supportive comments: Num. of comments supportive of the article's trustworthiness (range : $0-4$ ).

- Familiarity: Participant's familiarity of the article (range: $0-100$ ).

- Initial confidence: Participant's confidence in the initial trustworthiness rating prior to revealing user comments (range: 0 - 100).

- Gender: Participant's self-disclosed gender.

- Age: Participant's age (range: 20 - 59).

- Social media usage: Hours spent on social media per week by the participant (range: 1 $-30)$.

- User ID: A unique identifier assigned to each participant during the survey.

We used the $\mathrm{R}$ package lme4 to perform a generalised linear mixed-effects model (GLMM) analysis of the relationship between the aforementioned variables and participant conformity. A GLMM allows us to identify the effect of a set of predictor variables on an outcome variable (conformity) while following an arbitrary (i.e., possibly non-normal) distribution. We specified participant (User ID) as a random effect to account for individual differences in our model.

All statistically significant predictors included in the final model (following model selection through incremental addition of variables based on their predictive power) are shown in Table 1. We performed a likelihood ratio test with the null model and found that our model is statis- tically significant $\left(\chi^{2}=427.95, p<0.001\right)$ and explains $33.2 \%$ of the variance in accuracy $(R=$ $0.58, R^{2}=0.33$ ). To ensure the validity of the model, we then checked for the existence of multicollinearity. Our predictors report variance influence factors less than 1.10, well below the often-used threshold of 5 to detect multicollinearity [18].

Table 1. Effect of statistically significant predictors on participant conformity.

\begin{tabular}{lcc}
\hline Predictor & Coefficient & P-value \\
\hline Group size difference : Majority opinion (critical) & 1.00 & $<0.001$ \\
Group size difference & 0.63 & $<0.001$ \\
Initial confidence & -0.01 & $<0.001$ \\
\hline The sign of the coefficient $(+/-)$ denotes the direction of the relationship \\
between the predictor and conformity behaviour. Absolute value of the \\
coefficient determines the effect size.
\end{tabular}

We observed statistically significant main effects from group size difference (difference between the majority group size and the participant's group size) and the self-reported initial confidence level of participant. Moreover, the group size difference also interacted with the majority's opinion (either supportive or critical of the article) to display the highest effect on conformity. Next, we present a more detailed look of the significant features.

\section{Group size difference, Majority opinion and Initial confidence}

Our results reveal that participants were more inclined to conform to the majority as the size difference between the majority and themselves increased (despite the influence of other variables), signifying a main (positive) effect from group size difference on conformity. Furthermore, the impact of the group size difference on conformity heightened when participants were challenged by critical majorities than supportive majorities. As shown in Figure 2, while both lines display an upward trend, the likelihood of participants conforming to critical majorities is consistently higher than their likelihood of conforming to supportive majorities.

Furthermore, participants' confidence on their initial trustworthiness rating of an article (prior to seeing user comments) displayed a statistically significant negative effect on their conformity behaviour. When participants were less confident about their initial trustworthiness rating they were 


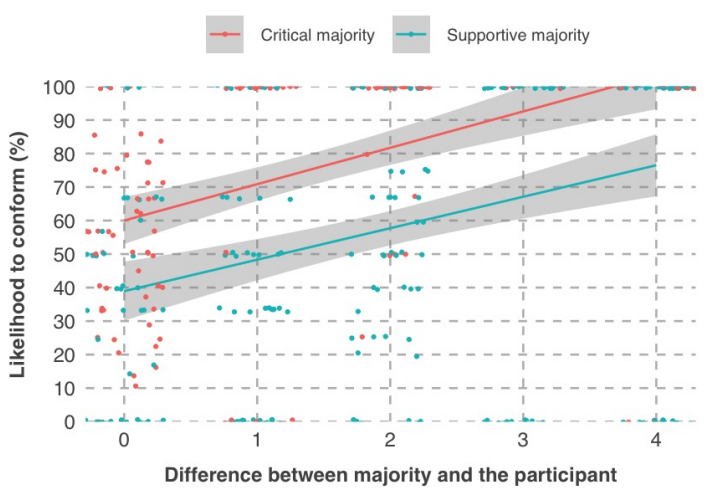

Figure 2. Participants' likelihood to conform to supportive and critical majorities as the group size difference increases from $0-4$.

more likely to be influenced by the majority's opinion. While the initial confidence level of participants ranged between $0-100$ in both non-conforming and conforming responses, mean initial confidence values were at $72.09(S D=$ $28.77)$ and $61.84(S D=25.64)$ respectively.

We did not note any significant effect from participants' reported familiarity of the post, gender, age or social media usage on their conformity behaviour during this analysis. Moreover, the total number of comments appearing in the post, the number of critical/supportive comments, or their presentation order had no notable influence on participant conformity.

\section{Participants' responses to news articles}

Upon establishing the presence of conformity in how people perceive trustworthiness of online news, we then investigated whether and how participants' conformity behaviour impact their response to news articles (i.e., their likelihood to react, comment, share, fact-check or report the Facebook post). We ran paired t-tests on the likelihood ratings reported by participants for each of the aforementioned response types, before and after viewing others' comments. This analysis was conducted across conforming and non-conforming responses separately, when participants were facing either supportive and critical majorities. Our results are summarised in Table 2.

We observe statistically significant mean differences among the before and after response likelihood ratings in conformity responses. Par-
Table 2. Mean difference between the likelihood ratings provided before and after seeing user comments for each response type.

\begin{tabular}{|c|c|c|c|c|}
\hline & \multicolumn{2}{|c|}{ Conforming Responses } & \multicolumn{2}{|c|}{ Non-conforming Responses } \\
\hline Response Type & Supportive Majority & Critical Majority & Supportive Majority & Critical Majority \\
\hline & $5.06(p<0.001)$ & $-11.20(p<0.001)$ & $-3.74(p=0.002)$ & $-1.61 \quad(p=0.232)$ \\
\hline $\begin{array}{l}\text { Comn } \\
\text { Share }\end{array}$ & $\begin{aligned} \mathbf{5 . 2 8}(p<0.001) \\
\mathbf{6 . 6 0}(p<0.001)\end{aligned}$ & $\begin{array}{l}-\mathbf{5 . 7 5}(p<0.001) \\
-\mathbf{8 . 8 1}(n<0.001)\end{array}$ & $\begin{array}{l}-0.83(p=0.480) \\
-2.02(p=026)\end{array}$ & $\begin{array}{l}-0.37(p=0.787) \\
-0.72(p=0.270\end{array}$ \\
\hline Fact-check & $7.32(p<0.0$ & -6.81 & -2.62 & $-7.91(p<0.001)$ \\
\hline Report & $-2.32(p=0.029)$ & $14.77(p<0.001)$ & $3.40(p=0.008)$ & $11.78(p<0.001)$ \\
\hline
\end{tabular}

ticipants who conformed to a majority supportive of an article's trustworthiness were significantly more inclined to fact-check, share, comment on and react on the Facebook post (respectively), after viewing user comments. Alternatively, participants who conformed to a majority critical of an article's trustworthiness reported higher inclination to report the post, while also lowering their inclination to react, share, fact-check and comment on the post.

Our results do not indicate statistically significant mean differences between the before and after response likelihood ratings in non-conforming responses against supportive majorities. However, when challenged by critical majorities participants reported higher inclination to report the post, and lower inclination to fact-check the post despite their non-conforming behaviour. The likelihood ratings reported for sharing, commenting and reacting on the post did not significantly change when faced with critical majorities in nonconforming responses.

\section{Discussion}

As human behaviour contributes more towards the dispersion of fake news than bots [8], its mitigation requires a thorough understanding of how people derive conclusions on a news article's trustworthiness. This study investigated how a combination of critical and supportive comments posted by others on a Facebook news article could influence subsequent readers' perception of the article's trustworthiness as well as their response to it.

Our findings confirm that readers frequently adjust their personal opinion on a news article's trustworthiness to agree with the opinion of a majority of previous readers, demonstrating conformity behaviour as seen in previous work [11]. As our study utilised combinations of both sup- 
portive and critical comments, we emphasise that a unanimous majority (critical or supportive) was not essential to trigger conformity, in contrast to previous observations [11]. Moreover, we note that participants were more likely to adopt the majority's opinion on an article's credibility as the number of comments reflecting the majority's sentiment (or the majority's size) increased. This is inline with observations from previous studies on online conformity [13], [14], [15]. More interestingly, the influence of the majority's size on participant conformity was higher when the majority was critical of an article's trustworthiness, than when the majority was supportive.

In addition, participants disregarded the majority's opinion when confident of their initial judgements, but were eager to adopt the majority's opinion when unsure of their initial judgements. Literature explains this behaviour as 'informational' conformity, where individuals conform to the majority presuming it to be 'correct' in uncertain situations (which is usually the case in online settings [13], [14], [15]). However, contrary to previous perceptions [11], an individual's familiarity with a news article or the time they spend on social media had no impact on their conformity behaviour.

Furthermore, readers who conformed to a critical majority were more inclined to take action against the dispersion of the news article (by reporting it) and were less inclined to contribute towards its further dispersion (by reacting, sharing and commenting on it), than readers who did not conform to the majority. Similarly, readers who conformed to a majority supportive of an article's trustworthiness were significantly more likely to share, comment and react on the news article to enable its further dispersion, than those who did not conform to a supportive majority.

Therefore, our observations not only confirm results of prior studies where the critical or supportive nature of user comments have been seen to influence how readers' perceive news articles as fake or real [11], but also provide insights on how conforming to others' opinions influence readers to align their responses to reflect the majority's (supportive or critical) opinion of an article. Hence, platforms should consider how user comments appearing underneath news articles on social media could be utilised to mitigate the dispersion of fake news, and encourage the dispersion of real news articles.

\section{Mitigating the dispersion of fake news on social media}

Social media platforms (e.g., Facebook) have been exploring how platform design could provide more context and facts on news articles to its readers, to assist them determine an article's credibility [10]. Our findings suggest that readers are receptive to others' comments and tend to mimic the majority's opinion on an article's trustworthiness derived through others' comments. This implies that displaying a filtered set of comments could be an effective approach to signal trustworthiness and credibility of news articles to readers and expose them to different perspectives on the topic, in comparison to displaying suggestions for related articles (as currently seen on Facebook).

Moreover, our results also have important implications on how platforms can effectively inform readers about fake news articles. Previous research note how disclaimers alerting readers of fake news had counterproductive results as they further entrenched personal beliefs of individuals [10]. In comparison, we recommend displaying a filtered set of critical user comments (unanimous or otherwise) underneath confirmed fake news articles, which is likely to encourage readers to adopt a critical opinion of its credibility, despite their personal perceptions due to conformity influences.

Conforming to the majority's opinion on an article's credibility also encouraged readers to align their responses with the majority's sentiment. Thus, by displaying a majority of critical user comments for potential fake news articles, platforms can mitigate their further dispersion. This is especially crucial as literature attributes the rapid dispersion of fake news to human behaviour [8]. Alternatively, for confirmed real news articles, displaying a majority of supportive comments could encourage further dispersion.

In conclusion, comments posted by readers on social media news articles could have untapped potential to assist platforms mitigate the dispersion of fake news. Our work is an initial step towards understanding how supportive and critical user comments trigger conformity in how subsequent users perceive and respond 
to news articles on social media. Further work could compare our results to other approaches currently used by social media platforms (such as related articles and disclaimers) to determine their comparative effectiveness. Moreover, while we considered the number and the presentation order of critical and supportive comments appearing on posts, we did not consider the popularity of each comment (i.e., the number of reactions and replies each comment received) which could also determine its influence. We encourage future work to explore these avenues to further expand our understanding on how user comments could be utilised to mitigate human-induced dispersion of fake news in social media.

\section{DEFERENCES}

1. E. Shearer and K. E. Matsa, "News use across social media platforms 2018," 2018.

2. H. Allcott and M. Gentzkow, "Social media and fake news in the 2016 election," Journal of economic perspectives, vol. 31, no. 2, pp. 211-236, 2017.

3. J. Weedon, W. Nuland, and A. Stamos, "Information operations and facebook," Retrieved from Facebook: https://fbnewsroomus. files. wordpress. com/2017/04/facebook-and-information-operations-v1. pdf, 2017.

4. H. J. Parkinson, "Click and elect: how fake news helped donald trump win a real election," The Guardian, vol. 14, 2016.

5. H. S. Yang, "The effects of the opinion and quality of user postings on internet news readers' attitude toward the news issue," Korean Journal of Journalism \& Communication Studies, vol. 52, no. 2, pp. 254-281, 2008.

6. E.-J. Lee and Y. J. Jang, "What do others' reactions to news on internet portal sites tell us? effects of presentation format and readers' need for cognition on reality perception," Communication research, vol. 37 , no. 6 , pp. 825-846, 2010.

7. C. Silverman and J. Singer-Vine, "Most americans who see fake news believe it, new survey says," BuzzFeed News, vol. 6, 2016.

8. S. Vosoughi, D. Roy, and S. Aral, "The spread of true and false news online," Science, vol. 359, no. 6380, pp. 1146-1151, 2018.

9. K. Shu, A. Sliva, S. Wang, J. Tang, and H. Liu, "Fake news detection on social media: A data mining perspective," ACM SIGKDD Explorations Newsletter, vol. 19, no. 1, pp. 22-36, 2017.
10. T. Lyons, "Replacing disputed flags with related articles," 2017.

11. J. Colliander, "'this is fake news": Investigating the role of conformity to other users' views when commenting on and spreading disinformation in social media," Computers in Human Behavior, vol. 97, pp. 202-215, 2019.

12. M. Deutsch and H. B. Gerard, "A study of normative and informational social influences upon individual judgment.," The Journal of Abnormal and Social Psychology, vol. 51, no. 3, pp. 629-636, 1955.

13. S. Wijenayake, N. van Berkel, V. Kostakos, and J. Goncalves, "Impact of contextual and personal determinants on online social conformity," Computers in Human Behavior, vol. 108, pp. 106-302, 2020.

14. S. Wijenayake, N. van Berkel, V. Kostakos, and J. Goncalves, "Measuring the effects of gender on online social conformity," Proceedings of the ACM on Human-Computer Interaction, vol. 3, no. CSCW, pp. 145:1-145:24, 2019.

15. S. Wijenayake, N. van Berkel, V. Kostakos, and J. Goncalves, "Quantifying the effect of social presence on online social conformity," Proceedings of the ACM on Human-Computer Interaction, vol. 4, no. CSCW, pp. 55:1-55:22, 2020.

16. Facebook, "What does most relevant mean on a facebook page post?," 2020.

17. E. Peer, J. Vosgerau, and A. Acquisti, "Reputation as a sufficient condition for data quality on amazon mechanical turk," Behavior research methods, vol. 46, no. 4, pp. 1023-1031, 2014.

18. J. F. Hair, W. C. Black, B. J. Babin, R. E. Anderson, and R. Tatham, Multivariate Data Analysis. New Jersey, NJ, USA: Pearson, 2010. 


\section{Chapter 9}

\section{Discussion}

Recently, different manifestations of social conformity have been reported in CMC-based groups with both positive and negative implications. As a result, there is a growing interest among researchers to better understand the dynamics of social conformity in CMC-based group settings. This thesis sets out to quantify online social conformity in terms of its contextual and personal determinants, identify its potential to generate both positive and negative outcomes, and propose design guidelines to appropriately control conformity influences in CMC-based group settings to mitigate its adverse effects. We present the original research contributions of this thesis in Chapters 4, 5, 6, 7 \& 8. In this chapter, we position our findings in relation to the conformity literature, reflect on how they answer the research questions identified in Chapter 1, and set out avenues for future work.

\subsection{Impact of Contextual Determinants on Online Social Conformity}

There is a plethora of prior work investigating contextual determinants of social conformity in physical groups that emphasise the significance of contextual factors in understanding conformity behaviour $[4,12,20,42,82,123]$. While some effort has been taken in CMC-based groups to replicate findings of these studies [28, 97, 106, 139, 176], we identify several research gaps with regard to effects of contextual determinants i.e., majority-minority group sizes, task objectivity and social presence - that need further investigation in CMC groups.

RQ 1: What is the impact of established contextual determinants of faceto-face conformity literature (majority-minority group composition, task objectivity, and the level of social presence) in triggering social conformity in CMC groups?

In Section 2.4, we note that there is a clear requirement for an in-depth analysis of different majority-minority group compositions on social conformity behaviour in CMC groups, that also accounts for the possibility of having multiple minorities of different sizes. Moreover, it is unclear if the impact of the majority-minority group composition on social conformity is moderated by other contextual determinants in CMC groups [4, 28, 106]. Hence, this thesis set out to systematically quantify how different majority-minority group compositions impact online conformity, in the presence of other contextual and personal determinants of conformity. 
In Chapter 4 we show that when the majority-minority group distribution is indicated using bar charts and percentages, users' tendency to conform is positively related to the size of the majority - regardless of the number and the size of the minority groups present, and other conformity determinants tested. Conversely, in Chapters 6, 7 \& 8 we note that when the presentation of the majority-minority group composition is less obvious (i.e. listing answers of supposed others, in contrast to indicating group sizes as percentages), it is the group size difference between the majority and the minority that determines conformity behaviour. In other words, as the difference between the majority and the minority (that supports user's initial response) increases, users feel more pressure to conform. Moreover, this effect is also independent of other contextual and personal determinants of conformity investigated in Chapters 6, 7 \& 8 - i.e., task objectivity, social presence, gender, age, self-confidence and personality. Hence, these findings provide strong empirical evidence that the positive relationship that has been reported between majority group size and conformity by prior work [28, 106, 176], can persist in diverse majority-minority group compositions - regardless of other conformity determinants. However, the disparity between our findings in Chapter 4 and Chapters 6, $7 \& 8$ imply that the presentation of the majority-minority group composition may moderate its effects in online groups.

Moreover, while prior work in face-to-face groups found higher conformity in tasks of subjective than objective nature [20], other studies report mixed findings in CMC groups $[78,97]$. Therefore, in Chapters $4 \& 7$ we examined user conformity in both objective (general knowledge) and subjective (opinion-based) quiz questions. We observe that in anonymous online groups, conformity is higher in objective than subjective questions - which stands in contrast to findings in physical conformity studies [20], and some CMC-based studies where users were not anonymous [97]. We further note that in the studies presented in Chapters $4 \& 7$, task objectivity had the largest effect on user conformity behaviour, and was independent of other personal and contextual determinants investigated i.e., majority-minority group size, social presence, gender, self-confidence and personality.

This discrepancy in findings with regard to task objectivity of physical and CMC groups can be explained by the inherent differences between the two contexts, and their consequences on user susceptibility to normative and informational influences. As per Perfumi et al. [134], anonymity and physical isolation in CMC groups, can trigger deindividuation effects that reduce user susceptibility to normative influences. This also explains why effects of task objectivity vary in anonymous $[78,179,181]$ and non-anonymous CMC settings [97]. Additionally, prior work note that informational influences persist, even in anonymous CMC settings [134] - a notion that our qualitative analysis repeatedly showed was a motivator behind conforming to objective quiz questions.

Furthermore, the literature shows that social presence - a contextual determinant that has been often investigated in terms of social context cues, interactivity, response visibility and humanness of peers - is positively related to users' tendency to conform in both physical $[5,42,47,60]$ and CMC [97, 103, 106, 111, 134, 162] groups. Chapter 7 of this thesis builds on prior work, to investigate potential interaction effects between three aspects of social presence in online groups i.e., user representation, interactivity 
Impact of Personal Determinants on Online Social Conformity

and response visibility - that have been previously investigated individually [97, 103, 106, 111]. Additionally, we examine if perceived social presence moderates effects of other contextual and personal conformity determinants in online groups i.e., majority-minority group sizes, task objectivity, gender and self-confidence.

We describe in Chapter 7 that a meaningful increase in social conformity was observed in the presence of both higher interactivity and response visibility - in addition to effects of majority-minority group size difference, task objectivity, and self-confidence. Furthermore, as none of the investigated aspects of social presence affected conformity individually, our findings imply that these aspects need to be examined simultaneously - contrary to what has been done in the literature [97, 103, 106, 111]. Furthermore, our qualitative data elaborate that higher social presence insinuated through higher interactivity and public responses made users re-evaluate how their non-conforming behaviour would be perceived by others in the group - suggesting effects of heightened normative influences. Hence, we provide empirical evidence that users' susceptibility to normative conformity can be enhanced by increasing perceived online social presence, in terms of interactivity and response visibility. Additionally, as the anonymous, generic vs. user-specific user representations showed no significant differences in user conformity, we argue that social context cues should either reveal user identity [42,60], or be used in tandem with experimentally induced perceptions of group membership [103] (as seen in prior work) to enhance social conformity.

\subsection{Impact of Personal Determinants on Online Social Conformity}

While personal determinants such as user gender, age, self-confidence and personality have been frequently investigated with relation to social conformity in physical groups [36, 39, 52, 72, 91, 98, 123, 132, 142], only a handful of studies have tested their effects in CMC groups [96, 102, 130, 134, 139, 188]. Moreover, the majority of the studies that examine effects of gender and age on social conformity have done so only from the perspective of the user who face social pressure situations [52, 91, 139, 188] and hence have not accounted for potential effects from the gender and age composition of "peers". This is critical because both gender and age of group members have been identified as indicators of their "status" within the group by prior work [47, 55], and thus has the potential to determine how receptive users are to their peers in both physical and $\mathrm{CMC}$ groups. This thesis examines the impact of personal determinants such as gender, age, self-confidence and personality on online social conformity, while also focusing on how gender and age related stereotypical perceptions of self/peer competency may add to these effects.

RQ 2: What is the impact of established personal determinants of face-toface conformity literature (gender, age, self-confidence and personality) in triggering social conformity in CMC groups? 
RQ 2 (a): How do gender and age based stereotypical perceptions of (peer and self) competency impact conformity behaviour in CMC groups?

While the literature indicate higher tendency among women than men to conform in face-to-face group settings $[47,52,98]$, these observations do not replicate in online groups $[139,188]$. Furthermore, while gender has been linked to stereotypical perceptions of self and peer competency in physical groups [185], it has only been investigated in relation to a "partner" (or a single peer) in online groups [101]. Therefore, in addition to accounting for potential gender differences in user conformity behaviour in Chapters 4, 5, 6, 7 \& 8, we investigate how being exposed to different gender compositions in the majority and minority groups affect user conformity in an online setting - in Chapter 6.

In line with prior work in CMC settings [139, 188], we do not find significant gender differences between men and women in how they respond to conformity pressures in the absence of gender cues of themselves and others (in Chapters 4, 5, 7 \& 8). However, in the presence of gender cues i.e. stereotypically gendered names and silhouette avatars (in Chapter 6), we note that both men and women are eager to conform to majorities with more men than women, in stereotypically masculine tasks. Conversely, in stereotypically feminine tasks they preferred majorities with more women than men. Additionally, this behaviour was more prominent in women than in men.

Hence, our findings have several implications. First, it is clear that gender differences in conformity that occur in physical groups as a result of socially imposed gender roles [47, 48], only manifest in CMC groups when gender identities of users can be inferred (as in Chapter 6). Moreover, findings of Chapter 6 show that in the presence of simple gender cues, people actively infer peer gender, and stereotypically perceive peer and self competency in stereotypically gendered tasks. Our qualitative data indicates that users expect such stereotypical conformity behaviour to help them reach the "correct" response to stereotypically gendered tasks. Thus, these findings imply that gender stereotypes can heighten effects of informational influences that trigger conformity in online groups. Additionally, as we note no gender effects with regard to non-stereotypical (or neutral) tasks, these effects should only activate in the presence of tasks that users perceive as gender-biased - which can vary cross-culturally.

Moreover, the impact of age on user conformity behaviour has not been systematically investigated in CMC groups - despite studies that recognise user age as a critical conformity determinant in physical settings [35, 36, 91, 94, 132]. Furthermore, prior work also imply that factors such as task type and users' awareness of peer age can moderate effects of user age on conformity $[91,94,132]$ - and hence should be analysed together. Therefore, in Chapter 5 we set out to examine how users from two distinct age groups Generations $\mathrm{X}$ and $\mathrm{Z}$ - respond to conformity pressures generated by peers belonging to the opposite age group, as they complete age-biased tasks through an online IM platform.

Chapter 5 presents findings that support the presence of strong age-stereotypical conformity in online groups - similar to the aforementioned effects of gender-stereotypical conformity in Chapter 6. More specifically, we found that both Generation X and Z users are more inclined to conform to opposing majorities that contain peers from the opposite 
age group, in tasks that they stereotypically perceive to align with the corresponding peer age group. Alternatively, when users stereotypically perceive a task to appeal to their own age group, they are significantly less receptive to an opposing majority. Additionally, the post-test survey data from this experiment indicate that observed agerelated stereotypical perceptions enhanced effects of traditional informational influences.

To the best of our knowledge, this is the first study to systematically investigate effects of user age and related stereotypes on online social conformity. We build on prior work with regard to physical groups that implied potential interactions between user and peer age, and the experimental task [91, 94, 132], to empirically establish that people derive stereotypical perceptions of self and peer competency based on age, that subsequently determine their receptiveness to conformity influences in online groups.

Furthermore, while face-to-face conformity literature reveals an inverse relationship between the experimentally induced self-confidence of users, and their subsequent conformity behaviour $[142,160]$, only a few studies have accounted for self-confidence as a potential determinant of conformity in CMC groups [96, 102]. Moreover, while these CMC-based studies confirm that lower self-confidence can heighten user conformity behaviour, they are inadequate to understand effects of self-confidence alongside other contextual and personal determinants. Thus, in the studies presented in Chapters 4, 5, 6, 7 $\& 8$, we systematically evaluated the relationship between user-reported self-confidence and conformity, across different CMC settings, alongside other contextual and personal determinants such as majority-minority group size, task objectivity, social presence, gender, age and personality.

Our findings across all five studies support that lower self-reported confidence leads to higher conformity behaviour in online groups - as previously reported in both physical $[142,160]$ and CMC $[96,102]$ groups. We also present qualitative evidence that rationalise this effect in terms of informational influences - i.e. users when unsure of their personal judgement, tend to perceive the majority to as an "additional source of information" to obtain the "correct" response, triggering conformity behaviour. Additionally, as this effect was independent of the other contextual and personal determinants investigated in this thesis, our findings signify the importance of accounting for effects of self-confidence when investigating conformity behaviour - even when it is not the main focus of an experiment.

Finally, this thesis also set out to determine if certain personality traits of individuals make them more or less susceptible to conformity influences in CMC groups. This was motivated by the fact that recent online conformity studies have taken an interest in quantifying conformity in terms of the Big-five personality traits [130, 134], but show mixed effects from the OCEAN traits (Openness, Conscientiousness, Extraversion, Agreeableness and Neuroticism) [84] on user conformity behaviour, and do not consider how they manifest alongside other conformity determinants. Moreover, these studies are not sufficient to understand why people with certain personality traits are less or more susceptible to conformity influences.

Therefore, in Chapter 4 we quantify effects of the Big-five personality traits on online user conformity. Our approach is novel in comparison to prior work, as we simultaneously account for effects of other contextual and personal conformity determinants such as majority-minority group sizes, task objectivity, gender and self-confidence. We found that 
users with higher Conscientiousness and Neuroticism conform more often than others irrespective of the effects of other conformity determinants. No other personality traits showed significant effects on user conformity. In other words, our findings show that individuals who are less emotionally stable (and hence show higher neuroticism) or show goal-oriented behaviour (or high conscientiousness) are more susceptible to conformity influences. These observations resonate with findings in physical conformity studies that positively correlate social anxiety $[109,119]$ and achievement motivation [157] with users' receptiveness to conformity influences. Furthermore, while the observed effects from Conscientiousness and Neuroticism contradict with prior findings in CMC groups [130, 134], we argue that this discrepancy is reasonable given the high informational influences reported in our study, in contrast to prior work in CMC groups where tasks that appeal to normative influences were used.

\subsection{Positive and Negative Outcomes of Online Social Conformity}

Contrary to beliefs of seminal face-to-face conformity studies [42], social conformity has been reported to elicit both positive outcomes (i.e. enhanced sense of community [154, 168]) and negative outcomes (i.e. undue pressure to conform to incorrect group judgements $[19,81])$ in CMC groups. Thus, to fully understand dynamics of online social conformity, and use this powerful social phenomenon to encourage prosocial behaviours in online groups effectively, acknowledging its potential for both positive and negative outcomes is important. Hence, this thesis set out to provide empirical evidence of potential positive and negative outcomes of online social conformity, in an attempt to further emphasise why conformity influences should be accounted for, when designing online group settings.

RQ 3: What are the potential positive and negative outcomes of online conformity?

In Chapter 6, we found that in the presence of gender cues (stereotypically gendered names and avatars), gender-stereotypical conformity behaviour led to more incorrect answers in gender-stereotypical quiz questions, in comparison to neutral questions. This is not surprising as the pilot study described in Chapter 6 reports neither men or women to be more competent in a certain type of questions (gender-stereotypical or otherwise). As a result, gender-stereotypical perceptions that heightened users' susceptibility to conformity - primarily for informational reasons - backfired.

Hence, when there is a possibility for people to stereotypically perceive their competency based on factors such as gender, age (see Chapter 2), and race [23, 27] - especially in the absence of direct indicators of self and peer competency in online groups - it is probable that conformity behaviour will result in adverse outcomes i.e. persuading users to accept and support incorrect judgements of the group's majority. 
In addition, Chapter 8 reveals that using social information (i.e. user comments posted by others evaluating article's trustworthiness) to trigger conformity in how people perceive trustworthiness of online news articles, can not only encourage people to overcome their personal prejudices of the said articles' credibility, but also persuade users to adjust how they respond to articles they perceive as fake or real. This is critical, as prior work has attributed the dispersion of fake news in online social media to actions of human users and not social bots [173]. Therefore, the ability to use conformity influences to encourage people to help the dissemination of real news articles, and avoid contributing to the dissemination of fake news articles in online social media has tremendous benefits.

\subsection{Accounting for Social Conformity in Platform Design}

This thesis aims to expand our understanding of the dynamics of conformity determinants, so that future CMC-based group settings can be designed to control conformity influences as per the requirements of the platform. More specifically, in group settings where independent or diverse user responses are welcome (e.g., online quizzing platforms, discussion forums [19]), the platform design itself should be equipped to discourage conformity behaviour. Conversely, in situations where conformity behaviour is desired to enhance a sense of community and encourage adherence to accepted group behaviours (e.g. online support groups $[154,168])$, the group platform can be designed to encourage user conformity.

We further note that literature shows that conveying social information through online platform design can enhance task performance [43] and engagement [56]. There are also reports that normative conformity can be encouraged through simple visual, textual, and interaction design elements of website design [168]. However, these findings are not explained in relation to conformity determinants and hence are inadequate to fully understand how conformity influences can be controlled through online platform design.

RQ 4: How can we embed conformity determinants in the design of CMC group platforms to control conformity influences appropriately?

In Chapters 5, $6 \& 7$ we present empirical evidence that certain design decision of an online platform can control conformity influences. For instance, embedding simple user cues such as usernames with birth years, first names of users and silhouette avatars resulted in age and gender stereotypical conformity behaviour in Chapters 5 and 6, respectively. Moreover, in Chapter 6 gendered avatars were seen to elicit higher stereotypical conformity than gendered names, implying that stronger cues can heighten such effects. Hence, in realistic online group settings where photographs of users and highly anthropomorphic avatars are used (e.g., [46, 67, 86, 140]), reported conformity effects would be more profound. 
Therefore, our findings present several implications worthy of consideration by designers of future online group settings. First, one should carefully consider if embedding user cues that can trigger stereotypical conformity is value-adding from the perspective of the users as well as the platform. For instance, gender and age related user cues can be relevant details in an online dating platform, but may not have the same significance in an online learning platform. Therefore, in situations where user cues are not necessary, we recommend using user representations that are devoid of user-specific cues i.e., animal avatars used by Google and identicons used by GitHub. This is further supported by the fact that people frequently perceive "gender-neutral" human-like avatars that are popularly used in online platforms as masculine [15] - and hence are not as effective in eliminating gender stereotypes. Alternatively, to avoid people from stereotypically inferring user competency based on irrelevant factors, we encourage embedding direct and reliable indicators of user competency - i.e., skill assessment tests in LinkedIn and badges used in Stack Overflow - in the platform design where relevant, to mitigate adverse effects of stereotypical user conformity behaviour in online groups.

Moreover, in Chapter 7 we show that the level of perceived online social presence can be manipulated through the level of interactivity and response visibility allowed in an online platform, and that higher social presence can enhance user susceptibility to social conformity influences - especially when the task appeals to normative influences. Therefore, we argue that platform designs that insinuate higher social presence can encourage social conformity when it leads to prosocial behaviours (e.g. in online support groups to enhance a sense of community and adhere to positive group norms [154]). Conversely, platforms designs that insinuate lower levels of online social presence can reduce the general receptiveness of users to social conformity influences - especially when conformity reportedly leads to adverse outcomes (e.g., in online group quizzes as seen in Chapter 6 and [19]).

Furthermore, while in Chapter 7 we enhanced the level of interactivity allowed between users through a text-based real time chat, and ensured that users' final judgements are publicly visible to all group member to improve response visibility, we emphasise that these factors can be enhanced through many other design features that we often see in online group settings i.e., a notification system that update users on others' behaviour on the platform [43]. Hence, more work is necessary to determine how interactivity and response visibility can manifest differently through online platform design, and whether they impose similar effects on social conformity behaviour.

On a different note, as social presence can also contribute towards user engagement and interaction [58, 73], user satisfaction and trust [71, 74], and platform attractiveness [73], the decision to control conformity influences by manipulating social presence should be taken after carefully considering the requirements of each platform. More work is required to understand how manipulating social presence, in an attempt to control online conformity behaviour could also impact the above aspects of a platform. Moreover, while this thesis sets forth several design guidelines based on empirical evidence on how certain conformity determinants can be embedded into online platform design to control conformity influences, further work is needed to validate these guidelines in realistic online group settings. 


\subsection{Future Directions of Online Social Conformity Research}

Next, we discuss several research avenues that are worthy of further investigation, particularly in CMC-based conformity studies. First, we emphasise the importance of taking precautions to mitigate experimenter-induced confounds that can impact results of social conformity experiments. Second, we highlight often overlooked determinants that are important to better understand the dynamics of conformity behaviour in CMC-based groups. Third, there is a lack of research that extends findings of controlled conformity studies to realistic CMC group settings. Finally, we call attention to the lack of literature that investigates the longevity of conformity behaviour in CMC groups, to differentiate between public compliance and private acceptance.

\subsubsection{Tackling the Experimenter Effect}

The "experimenter effect" is described as a participant's tendency to alter their behaviour in the presence of an experimenter, resulting in unnatural behaviour and hence invalid research outcomes [133]. More specifically, participants may adjust their behaviour during an experiment to align with subtle cues they extract from the experimenter regarding their objectives behind the experiment [174, 178], or even in response to certain socio-demographic features of the experimenter such as - gender, age and race [41, 107, 184]. Therefore, such experimenter effects can introduce confounding influences in conformity studies. For instance, if a participant somehow senses that the experimenter expects to see conformity behaviour, they may feel the need to do so, to produce "better" (but unnatural) study results. Furthermore, as literature also indicates that completely removing the experimenter can also cause insincere and satisficing answers, researchers have used bots in the place of experimenters in CMC groups [171]. More specifically, they argue that bots can potentially facilitate a human experimenter's conversational interactivity, without unconsciously exposing subjects to user cues or experimental details that can moderate their behaviour [178].

Several conformity studies have used bots to replace human experimenters in both physical and CMC settings. In physical groups, conformity studies that investigated effects of non-human (robotic) peers have used "experimenter" robots to control the flow of the experimental task [146, 147]. For instance, Salomons et al. [146] reported using a robot instead of a human experimenter to display each experimental trial, and show initial and final responses of the group members at the end of each trial. The authors argue that using a human experimenter in this setup can generate experimenter effects, potentially overpowering any influence of the robotic peers. Similarly, we used chatbots in Chapters 4, 5, $6 \&$ 7, to overcome possible experimenter effects. For example, in Chapters 4 $\& 7$ we used a "QuizBot" to assist participants in familiarising themselves with the online quizzing tool used for the experiment, and to provide step-by-step instructions on how to complete the quiz without any involvement from the researchers. Our qualitative findings indicate that the use of a bot was well-received by the participants, who also preferred receiving instructions from a bot in comparison to reading them on the screen 
or on paper. Moreover, a similar bot was used in Chapters $5 \& 6$ to investigate the effects of age, gender and related stereotypes on online user conformity behaviour, where the bot was used to train participants, without bringing attention to the experimenters' age and gender details, to minimise potential confounding effects.

We emphasise that researchers need to be mindful about how the bots are designed, if they are to be used as a mechanism to minimise experimenter effects in conformity studies. More specifically, the level of anthropomorphism and conversational capabilities of a bot can determine its effectiveness in mitigating experimenter effects [178]. As participants have often regarded computers as social actors in conformity studies and have responded similarly to human peers $[101,186]$, highly anthropomorphic bots with prominent user cues (related to gender, race, etc.) may induce experimenter effects similar to humans [178].

\subsubsection{Overlooked Determinants of Social Conformity}

Often overlooked determinants of social conformity - such as the cultural background of users [29, 126] and their cultural perceptions - require further investigation in future conformity studies. While there has been some effort to compare conformity behaviour between individuals coming from individualistic and collectivist national cultures (e.g., Cinnirella and Green [29] compared conformity behaviour between European vs. East Asian students), a majority of conformity studies have used participants coming from WEIRD (Western, Educated, Industrialised, Rich and Democratic) communities which have been reported to skew research findings with regard to human nature [85]. Importantly, CMC groups are inherently distributed and are more likely to contain culturally diverse cohorts of users when compared to physical groups. Hence, the findings we report in Chapter 6 with regard to the effects of gender and related stereotypes on online conformity, with participants primarily coming from a Western country, might not replicate cross-culturally due to different cultural norms. Hence, going forward CMCbased conformity research should focus on extending reported findings to non-WEIRD communities and ensure diversity in recruited participants, to accurately reflect the user groups that often use CMC groups for interpersonal relations.

Furthermore, despite increasing reports of cultural stereotypes moderating user behaviours in CMC settings [10,33], not much effort has been invested into understanding their effects on social conformity. Furthermore, as Eagly and Chrvala [47] indicate that certain user cues are often stereotypically perceived as indicators of user competency and status in physical groups, it is probable that these stereotypes also translate to CMC groups. For instance, in Chapters $5 \& 6$ we found that age and gender stereotypical perceptions can impact conformity behaviour in CMC groups. Thus, it is highly likely that other personal traits of individuals i.e., their cultural background, can also induce stereotypical conformity in CMC settings.

Therefore, future conformity studies can investigate whether and how cultural stereotypes occur in CMC groups, what factors contribute toward such behaviour, as well as their impact on the social dynamics of online groups. In addition, it is also important to analyse such stereotypes in relation to the nature of the experimental task and stereotypical user cues, to identify potential interactions. For instance, in Chapters 5 
\& 6 we observed age and gender stereotypical conformity in age and gender-biased quiz questions respectively, but not in neutral quiz questions based on general knowledge. This implies that stereotypes may only trigger conformity in the presence of tasks that appeal to them.

Additionally, while simple user cues were sufficient to trigger the reported stereotypical perceptions in Chapters $5 \& 6$, we found that richer user cues tend to generate stronger stereotypical behaviour in Chapter 6. Thus, while cultural stereotypes have been reported to occur even in the presence of first names of online users [33], we can reasonably expect them to be more profound in the presence of richer user representations such as photographs - where cultural cues are more obvious. Therefore, future work should also consider how the strength of cultural stereotypes might vary in different user representations. Additionally, they can also evaluate the effectiveness of strategies proposed in Section 9.4 to mitigate stereotypical conformity in CMC settings.

\subsubsection{Realistic Group Settings}

A significant $96.4 \%$ of the research articles analysed in our literature review used either confederates or simulations to generate an artificial group majority. Only four studies - two each in physical $[151,185]$ and CMC [111, 136] groups - have investigated conformity by exposing subjects to naturally occurring group majorities, without any experimental manipulations. Moreover, the majority of CMC conformity studies are conducted in laboratory settings that do not accurately represent realistic CMC-based group environments, using experimental tasks that are not representative of naturally occurring social pressure situations (e.g., Perfumi et al. [134] investigated conformity behaviour in subjects who were in a laboratory with a maximum of three experimenters, as they completed Asch's line judgement task online, with computer-simulated peers). Hence, it is possible that findings of prior laboratory based conformity studies do not directly translate to realistic CMC groups settings.

Therefore, more effort is necessary to investigate how findings with regard to effects of conformity determinants in controlled conformity studies replicate in realistic CMC groups that do not use confederates or simulations to create social pressure. For example, Maruyama et al. [111] reports a study where conformity in how subjects vote in a political election was investigated by exposing the subjects to a naturally occurring political debate on Twitter - where the majority's opinion was based on the tweets posted by the Twitter community. The study also analysed effects of interactivity on conformity behaviour by instructing subjects to either actively interact with the tweets, or simply observe them. By doing so, the authors confirmed that the level of interactivity between the subject and their online correspondents show a positive effect on their tendency to conform, when subjects are exposed to organic conformity influences occurring in a realistic CMC environment.

Furthermore, group settings in CMC environments (e.g., social networks, discussion forums) are often larger in size having thousands of users in some situations, in comparison to face-to-face group settings [59]. However, the overall group sizes used for CMC based conformity studies - which is often between 2-11 users [102, 105, 176] - do not correctly reflect the size of naturally occurring CMC based groups. While, 
using smaller group sizes may have been appropriate in initial CMC-based studies that primarily focused on understanding how effects of conformity determinants translate from physical to CMC groups, these studies are not sufficient to determine if these effects will persist across larger groups. Furthermore, while larger CMC groups are likely to further enhance deindividuation effects and a sense of anonymity in users that can reduce their susceptibility to conformity influences as previously reported by Perfumi et al. [134] and Kim and Park [89], these assumptions are yet to be empirically confirmed in CMC groups with larger cohorts of users.

Additionally, how the majority-minority group composition is signalled to users in CMC groups can also vary from one platform to the next (e.g. discussion forums such as Stack Overflow or Quora may signal the "popular" opinion in terms of upvotes, whereas an online poll can indicate the same, as percentages of users agreeing with each poll option). Similar differences were found where the group composition was insinuated to users by sequentially displaying responses of each user in Chapters 6, 7 $\& 8$, and alternatively by using visualisations such as bar charts in Chapter 4 . However, our findings are not sufficient to determine if different signals used to indicate group composition can determine users' receptiveness to conformity pressures in addition to the majority-minority composition. Moreover, it is probable that the effectiveness of such indicators can also vary if the overall size of the group increases significantly details of which are still unclear.

Therefore, we encourage future conformity studies to focus more on assessing the ecological validity of findings put forward by prior work. It is particularly important to investigate these questions in realistic CMC groups, that are considerably different to the laboratory based CMC group settings that have been used in prior conformity studies [134] - including the studies presented in Chapters 4, 6 \& 7. Additionally, we acknowledge that the controlled nature of the study designs used in this thesis may have affected the ecological validity of certain findings. For instance, often in realistic online group settings user cues related to culture, gender identity and age can manifest simultaneously to impact user conformity behaviour - which was not accounted for by the experiments presented in this thesis. However, we further emphasise that investigating conformity in uncontrolled, realistic CMC groups is considerably more challenging, as it requires careful experimental design to account for potential consequences of the uncontrolled nature of the study. For example, if the majority-minority group compositions are allowed to manifest organically in a group of participants, it is difficult to ensure that all participants will be in a minority for the experimental tasks. Furthermore, as potential confounds can also occur between conformity determinants as the uncontrolled nature may not allow for these to be controlled, analysing and interpreting results of such studies can also be more complex (e.g., when using photographic user representations instead of usernames with birth years or gendered avatars, user conformity can be impacted by cues such as culture, in addition to age and gender as seen in Chapters $5 \& 6$ ). 


\subsubsection{Levels of Conformity: Compliance, Identification and Internalisation}

To understand the true implications of conforming behaviour on an individual's personal opinions and behaviour, it is vital to recognise the "level of conformity" that has occurred. There are three distinct levels of conformity - compliance, identification and internalisation [87, 90]. "Compliance" is described as superficial, public conformity, without a private change in attitude or behaviour. In other words, an individual who does not believe in the group's judgement may still adjust their public opinion in the presence of an opposing majority, to achieve a favourable reaction from the group, to avoid disapproval or to gain a specific reward. Furthermore, "identification" occurs when an individual changes their opinions and behaviours to appear similar to a specific group. In such a situation, the person accepts the group's response without actually considering its content, because it is associated with the desired group relationship. Finally, if an individual shows both public and private conformity, because they believe the group's judgement to be accurate, useful or congenial, it is referred to as "internalisation" - which is the highest level of conformity. Behaviours and opinions adopted in this nature, often end up being integrated to a person's own value system, and thus results in private conformity.

Therefore, it is clear that the longevity of conformity behaviour depends on the level of conformity that a person demonstrates in response to social pressure. However, only a few studies have reported investigating the level of conformity induced during the experiment. For instance, in physical groups, Yarnold, Grimm, and Mueser [187] described that individuals who conformed to the responses of three unanimous confederates in a series of visual perceptual tasks, often reverted their responses when they completed the tasks by themselves, immediate after the group situation. In other words, the conformity behaviour induced in this Asch-like situation shows only public compliance in the presence of social pressure. Furthermore, a CMC-based conformity study by Laporte, Nimwegen, and Uyttendaele [97] reported that subjects who completed a set of factual, moral, and political questions in the presence of confederates and subsequently by themselves, showed public compliance in subjective tasks (moral/political questions), but not in objective tasks (factual questions). They argue that in objective questions conformity may have been triggered due to informational influences, which could have encouraged subjects to not only conform to the majority but also to privately accept (or internalise) the majority's answer as "correct". Alternatively, in subjective questions where there is no clear "correct" answer, public conformity can be a result of normative influences, that diminish when social pressure is removed.

Therefore, we note that understanding when and why different levels of conformity occur can greatly expand our understanding of the true consequences of conformity behaviour. More specifically, understanding the longevity of social conformity influences can assist researchers to determine when and where its effects are strongest and hence requires more attention. However, prior work on this regard is insufficient to fully determine what factors induce public compliance, identification, and private conformity. Therefore, we encourage future conformity studies to determine what level of conformity occurs within their experiment, using an experimental design that can reasonably 
distinguish between each level of conformity. With that being said, this is can be quite challenging as it requires participants to repeat the experimental task at least twice. Moreover, in situations where participants are required to repeat the experimental tasks immediately after being exposed to the group situation, it is highly likely that they provide satisficing answers due to fatigue. Alternatively, if participants are asked to repeat the tasks after a longer time interval (e.g. 2 weeks), researcher will have to put in extra effort to ensure participant retention. 


\section{Chapter 10}

\section{Conclusion}

This thesis investigates the dynamics of social conformity in online group settings, to inform the design of future online group settings that account for social conformity influences. To this end, we first systematically quantify the effects of several popular contextual and personal determinants of conformity in face-to-face groups, in different online group settings. Our findings show significant effects from all contextual and personal determinants analysed, which includes - majority and minority group sizes, task objectivity, social presence, gender, age, self-confidence and personality. Thus, online social conformity is a function of both its contextual and personal determinants, and hence should be examined and understood as such.

Next, we analyse outcomes of social conformity influences in online group settings, revealing that this social phenomenon has both positive and negative implications. On one hand, online social conformity influences can be used to encourage prosocial behaviours in social media (see Chapter 8). Conversely, social conformity influences when amplified by stereotypical perceptions - can motivate individuals in online groups to accept incorrect information supported by a majority as the truth (see Chapter 6). Hence, social conformity should not be cast aside as a negative influence, and instead should be accounted for - when designing online group settings.

We also present a set of guidelines to inform the design of the next generation of online social platforms, that can not only mitigate adverse effects of social conformity influences, but also capitalise on this social phenomenon when possible. In Chapters 5, 6 \& 7 we indicate how certain contextual and personal determinants of social conformity can manipulate perceived pressure to conform in online groups. For instance, we show that higher perceived social presence and stronger gender cues can heighten our susceptibility to social conformity influences, and vice versa. Having said that, as outcomes of social conformity differ from one platform to the next, how our findings can be used to encourage prosocial behaviour and/or mitigate adverse effects of conformity influences is up to the discretion of the designers of a specific platform. Moreover, future work can systematically investigate if these conformity determinants can be realistically manipulated through online platform design to control social conformity influences to encourage prosocial user behaviours.

The thesis also outlines a set of research avenues worthy of the consideration for future conformity research. First, despite our efforts to test conformity in both laboratory and remote studies that mimic real-world online group settings, these studies exposed users to artificially induced social pressure situations, and potential experimenter effects - and hence are not entirely representative of realistic scenarios. Additionally, more work is needed to explore effects of other conformity determinants i.e. culture - that are often overlooked by prior work, but are critical to the dynamics of social conformity in online 
groups that connect diverse cohorts of users. Finally, there is also a lack of research that explores the longevity of social conformity behaviour that manifest in online group settings - which is useful to determine its reach in our everyday lives. 


\section{References}

[1] Abrams, D., Wetherell, M., Cochrane, S., Hogg, M. A., and Turner, J. C. "Knowing what to think by knowing who you are: Self-categorization and the nature of norm formation, conformity and group polarization”. In: British journal of social psychology vol. 29.no. 2 (1990), pp. 97-119. DOI: https://doi.org/10.1111/j.2044-8309.1990.tb00892.x.

[2] Adams, A., Lunt, P., and Cairns, P. “A qualititative approach to HCI research”. In: Research Methods for Human-Computer Interaction (2008), pp. 138-157.

[3] Allen, V. L. and Crutchfield, R. S. "Generalization of experimentally reinforced conformity." In: The Journal of Abnormal and Social Psychology vol. 67.no. 4 (1963), pp. 326-333. Dor: https://doi.org/10. 1037/h0042074.

[4] Allen, V. L. and Levine, J. M. "Consensus and conformity”. In: fournal of Experimental Social Psychology vol. 5.no. 4 (1969), pp. 389-399. Dor: https://doi.org/10.1016/0022-1031(69)90032-8.

[5] Allen, V. L. and Levine, J. M. "Social support and conformity: The effect of response order and differentiation from the group”. In: British fournal of Social and Clinical Psychology vol. 10.no. 2 (1971), pp. 181-184. DoI: https://doi.org/10.1111/j.2044-8260.1971.tb00731.x.

[6] Allen, V. L. and Levine, J. M. "Social support and conformity: The role of independent assessment of reality”. In: Journal of experimental social psychology vol. 7.no. 1 (1971), pp. 48-58. DoI: https: //doi.org/10.1016/0022-1031(71)90054-0.

[7] Allen, V. L. and Levine, J. M. “Social support, dissent and conformity". In: Sociometry (1968), pp. 138-149. DOI: https://doi.org/10.2307/2786454.

[8] Allen, V. L. and Newtson, D. “Development of conformity and independence." In: Fournal of Personality and Social Psychology vol. 22.no. 1 (1972), pp. 18-30. Dor: https://doi.org/10.1037/ h0032386.

[9] Allport, G. W. “A test for ascendance-submission.” In: The fournal of Abnormal and Social Psychology vol. 23.no. 2 (1928), pp. 118-136. DoI: https://doi.org/10.1037/h0074218.

[10] Araújo, C. S., Meira, W., and Almeida, V. "Identifying Stereotypes in the Online Perception of Physical Attractiveness”. In: Social Informatics. Ed. by Spiro, E. and Ahn, Y.-Y. Cham: Springer International Publishing, 2016, pp. 419-437. DoI: https://doi.org/10.1007/978-3-319-47880-7_26.

[11] Asch, S. E. “Groups, leadership and men”. In: Oxford, England: Carnegie Press, 1951. Chap. Effects of group pressure upon the modification and distortion of judgements, pp. 177-190.

[12] Asch, S. E. "Opinions and social pressure". In: Scientific American vol. 193.no. 5 (1955), pp. 31-35. DOI: https://www.jstor.org/stable/24943779.

[13] Atkinson, J. W., Heyns, R. W., and Veroff, J. "The effect of experimental arousal of the affiliation motive on thematic apperception." In: The fournal of Abnormal and Social Psychology vol. 49.no. 3 (1954), pp. 405-410. DOI: https://doi.org/10.1037/h0053499.

[14] Azungah, T. "Qualitative research: deductive and inductive approaches to data analysis". In: Qualitative Research fournal vol. 18.no. 4 (2018), pp. 383-400. DoI: https://doi.org/10.1108/QRJ-D18-00035.

[15] Bailey, A. H. and LaFrance, M. "Anonymously male: Social media avatar icons are implicitly male and resistant to change”. In: Cyberpsychology: Journal of Psychosocial Research on Cyberspace vol. 10.no. 4 (2016). DOI: https://doi.org/10.5817/CP2016-4-8. 
[16] Baron, R. S., Vandello, J. A., and Brunsman, B. "The forgotten variable in conformity research: Impact of task importance on social influence." In: fournal of personality and social psychology vol. 71.no. 5 (1996), pp. 915-927. DoI: https://doi.org/10.1037/0022-3514.71.5.915.

[17] Barron, F. “An ego-strength scale which predicts response to psychotherapy." In: fournal of Consulting Psychology vol. 17.no. 5 (1953), pp. 327-333. DoI: https://doi.org/10.1037/h0061962.

[18] Beloff, H. "Two forms of social conformity: acquiescence and conventionality." In: The fournal of Abnormal and Social Psychology vol. 56.no. 1 (1958), pp. 99-104. Dor: https://doi.org/10.1037/ h0046604.

[19] Beran, T., Drefs, M., Kaba, A., Al Baz, N., and Al Harbi, N. "Conformity of responses among graduate students in an online environment”. In: The Internet and Higher Education vol. 25 (2015), pp. 63-69. DOI: https://doi.org/10.1016/j.iheduc.2015.01.001.

[20] Blake, R. R., Helson, H., and Mouton, J. S. “The Generality of Conformity Behavior as a Function of Factual Anchorage. Difficulty of Task, and Amount of Social Pressure". In: Journal of Personality vol. 25.no. 3 (1957), pp. 294-305. Dor: https://doi.org/10.1111/j.1467-6494.1957.tb01528.x.

[21] Blandford, A., Furniss, D., and Makri, S. "Qualitative HCI research: Going behind the scenes". In: Synthesis lectures on human-centered informatics vol. 9.no. 1 (2016), pp. 1-115. DOI: https: //doi.org/10.2200/S00706ED1V01Y201602HCI034.

[22] Bleize, D. N., Anschutz, D. J., Tanis, M., and Buijzen, M. "The effects of group centrality and accountability on conformity to cyber aggressive norms: Two messaging app experiments”. In: Computers in human behavior vol. 120 (2021), p. 106754. DoI: https://doi.org/10.1016/j.chb.2021. 106754.

[23] Bliuc, A.-M., Faulkner, N., Jakubowicz, A., and McGarty, C. "Online networks of racial hate: A systematic review of 10 years of research on cyber-racism”. In: Computers in Human Behavior vol. 87 (2018), pp. 75-86. DoI: https://doi.org/10.1016/j.chb.2018.05.026.

[24] Brandstetter, J., Rácz, P., Beckner, C., Sandoval, E. B., Hay, J., and Bartneck, C. “A peer pressure experiment: Recreation of the Asch conformity experiment with robots”. In: 2014 IEEE/RSf International Conference on Intelligent Robots and Systems. 2014, pp. 1335-1340. DoI: 10.1109/ IROS.2014.6942730.

[25] Braun, V. and Clarke, V. "Thematic analysis." In: Research designs: Quantitative, qualitative, neuropsychological, and biological (2012), pp. 57-71. Dor: https://doi.org/10.1037/13620-004.

[26] Braun, V. and Clarke, V. "Using thematic analysis in psychology". In: Qualitative research in psychology vol. 3.no. 2 (2006), pp. 77-101. DoI: https://doi.org/10.1191/1478088706qp063oa.

[27] Burkhalter, M. "Reading race online". In: Communities in cyberspace (1999), pp. 60-75. DoI: https: //doi.org/10.1126/science.aap9559.

[28] Campbell, J. D. and Fairey, P. J. "Informational and normative routes to conformity: The effect of faction size as a function of norm extremity and attention to the stimulus." In: fournal of personality and social psychology vol. 57.no. 3 (1989), pp. 457-468. Dor: https://doi.org/10.1037/00223514.57.3.457.

[29] Cinnirella, M. and Green, B. "Does 'cyber-conformity’vary cross-culturally? Exploring the effect of culture and communication medium on social conformity". In: Computers in Human Behavior vol. 23.no. 4 (2007), pp. 2011-2025. DOI: https://doi.org/10.1016/j.chb.2006.02.009.

[30] Cocos, A., Qian, T., Callison-Burch, C., and Masino, A. J. "Crowd control: effectively utilizing unscreened crowd workers for biomedical data annotation”. In: Fournal of biomedical informatics vol. 69 (2017), pp. 86-92. Dor: https://doi.org/10.1016/j.jbi.2017.04.003.

[31] Coleman, J. F., Blake, R. R., and Mouton, J. S. “Task difficulty and conformity pressures.” In: The Journal of Abnormal and Social Psychology vol. 57.no. 1 (1958), pp. 120-122. DoI: https: //doi.org/10.1037/h0041274. 
[32] Colliander, J. “"This is fake news”: Investigating the role of conformity to other users' views when commenting on and spreading disinformation in social media”. In: Computers in Human Behavior vol. 97 (2019), pp. 202-215. DoI: https://doi.org/10.1016/j.chb.2019.03.032.

[33] Conaway, W. and Bethune, S. "Implicit Bias and First Name Stereotypes: What Are the Implications for Online Instruction?.” In: Online Learning vol. 19.no. 3 (2015), pp. 162-178.

[34] Costanzo, P. R. "Conformity development as a function of self-blame." In: Journal of Personality and Social Psychology vol. 14.no. 4 (1970), pp. 366-374. Dor: https://doi.org/10.1037/h0028983.

[35] Costanzo, P. R., Reitan, H. T., and Shaw, M. E. "Conformity as a function of experimentally induced minority and majority competence”. In: Psychonomic Science vol. 10.no. 10 (1968), pp. 329-330.

[36] Costanzo, P. R. and Shaw, M. E. “Conformity as a function of age level”. In: Child development (1966), pp. 967-975. DoI: https://doi.org/10.2307/1126618.

[37] Crowne, D. P. and Liverant, S. "Conformity under varying conditions of personal commitment." In: The fournal of Abnormal and Social Psychology vol. 66.no. 6 (1963), pp. 547-555. Dor: https: //doi.org/10.1037/h0048942.

[38] Crowne, D. P. and Marlowe, D. "A new scale of social desirability independent of psychopathology." In: Journal of consulting psychology vol. 24.no. 4 (1960), pp. 349-354. DoI: https://doi.org/10.1037/ h0047358.

[39] Crutchfield, R. S. "Conformity and character." In: American psychologist vol. 10.no. 5 (1955), pp. 191198. DOI: https://doi.org/10.1037/h0040237.

[40] Daniel, M. and Peter, H. "The effects of information and social conformity on opinion change". In: PLoS ONE vol. 13.no. 5 (2018). DoI: https://doi.org/10.1371/journal.pone.0196600.

[41] Davis, D. W. and Silver, B. D. "Stereotype threat and race of interviewer effects in a survey on political knowledge”. In: American fournal of Political Science vol. 47.no. 1 (2003), pp. 33-45. DOI: https://doi.org/10.1111/1540-5907.00003.

[42] Deutsch, M. and Gerard, H. B. "A study of normative and informational social influences upon individual judgment.” In: The fournal of Abnormal and Social Psychology vol. 51.no. 3 (1955), pp. 629-636. DOI: https://doi.org/10.1037/h0046408.

[43] Diner, D., Nakayama, S., Nov, O., and Porfiri, M. "Social signals as design interventions for enhancing citizen science contributions”. In: Information, Communication \& Society vol. 21.no. 4 (2018), pp. 594611. DoI: https://doi.org/10.1080/1369118X.2017.1299779.

[44] Downs, J. S., Holbrook, M. B., Sheng, S., and Cranor, L. F. “Are your participants gaming the system? Screening Mechanical Turk workers”. In: Proceedings of the SIGCHI conference on human factors in computing systems. 2010, pp. 2399-2402. DOI: https://doi.org/10.1145/1753326.1753688.

[45] Duval, S. "Conformity on a visual task as a function of personal novelty on attitudinal dimensions and being reminded of the object status of self”. In: Fournal of Experimental Social Psychology vol. 12.no. 1 (1976), pp. 87-98. DoI: https://doi.org/10.1016/0022-1031(76)90088-3.

[46] Döring, N., Reif, A., and Poeschl, S. "How gender-stereotypical are selfies? A content analysis and comparison with magazine adverts”. In: Computers in Human Behavior vol. 55 (2016), pp. 955-962. DOI: https://doi.org/10.1016/j.chb.2015.10.001.

[47] Eagly, A. H. and Chrvala, C. "Sex differences in conformity: Status and gender role interpretations". In: Psychology of Women Quarterly vol. 10.no. 3 (1986), pp. 203-220. DOI: https://doi.org/10.1111/j. 1471-6402.1986.tb00747.x.

[48] Eagly, A. H., Wood, W., and Fishbaugh, L. "Sex differences in conformity: Surveillance by the group as a determinant of male nonconformity." In: Journal of Personality and Social Psychology vol. 40.no. 2 (1981), pp. 384-394. DOI: https://doi.org/10.1037/0022-3514.40.2.384.

[49] Edwards, A. L. "Edwards personal preference schedule". In: Psychological Corporation (1954). 
[50] Endler, N. S. "Conformity analyzed and related to personality". In: The fournal of Social Psychology vol. 53.no. 2 (1961), pp. 271-283. DOI: https://doi.org/10.1080/00224545.1961.9922125.

[51] Endler, N. S., Coward, T. R., and Wiesenthal, D. L. "The effects of prior experience with a task on subsequent conformity to a different task". In: The fournal of Social Psychology vol. 95.no. 2 (1975), pp. 207-219. DOI: https://doi.org/10.1080/00224545.1975.9918706.

[52] Endler, N. S., Minden, H. A., and North, C. "The effects of reinforcement and social approval on conforming behaviour”. In: European fournal of Social Psychology vol. 3.no. 3 (1973), pp. 297-310. DOI: https://doi.org/10.1002/ejsp.2420030308.

[53] Endler, N. S., Wiesenthal, D. L., Coward, T., Edwards, J., and Geller, S. H. "Generalization of relative competence mediating conformity across differing tasks”. In: European fournal of Social Psychology vol. 5.no. 3 (1975), pp. 281-287. DoI: https://doi.org/10.1002/ejsp.2420050302.

[54] Endler, N. S., Wiesenthal, D. L., and Geller, S. H. "The generalization of the effects of agreement and correctness on relative competence mediating conformity.” In: Canadian fournal of Behavioural Science/Revue canadienne des sciences du comportement vol. 4.no. 4 (1972), pp. 322-329. DoI: https: //doi.org/10.1037/h0082317.

[55] Fard, S. A. "Effect of gender and social status on conformity." In: Psychological Research vol. 13.no. 1 (2010), pp. 30-50.

[56] Farzan, R., Dabbish, L. A., Kraut, R. E., and Postmes, T. "Increasing Commitment to Online Communities by Designing for Social Presence”. In: Proceedings of the ACM 2011 Conference on Computer Supported Cooperative Work. CSCW '11. New York, NY, USA: Association for Computing Machinery, 2011, pp. 321-330. DoI: https://doi.org/10.1145/1958824.1958874.

[57] Fenigstein, A., Scheier, M. F., and Buss, A. H. "Public and private self-consciousness: Assessment and theory." In: Journal of consulting and clinical psychology vol. 43.no. 4 (1975), pp. 522-527. DOI: https://doi.org/10.1037/h0076760.

[58] Fortin, D. R. and Dholakia, R. R. "Interactivity and vividness effects on social presence and involvement with a web-based advertisement”. In: Journal of business research vol. 58.no. 3 (2005), pp. 387-396. DOI: https://doi.org/10.1016/S0148-2963(03)00106-1.

[59] Garton, L., Haythornthwaite, C., and Wellman, B. "Studying Online Social Networks". In: Journal of Computer-Mediated Communication vol. 3.no. 1 (June 1997). JCMC313. DoI: https://doi.org/10. 1111/j.1083-6101.1997.tb00062.x.

[60] Gerard, H. B. "Conformity and commitment to the group." In: The fournal of Abnormal and Social Psychology vol. 68.no. 2 (1964), pp. 209-211. DoI: https://doi.org/10.1037/h0045912.

[61] Gergen, K. J. and Bauer, R. A. "Interactive effects of self-esteem and task difficulty on social conformity." In: Journal of personality and social psychology vol. 6.no. 1 (1967), pp. 16-22. DOI: https://doi.org/10.1037/h0021216.

[62] Giannini, M., Pannocchia, L., Grotto, L., and Gori, A. "A measure for counseling: the five factor adjective short test (5-fast)”. In: Couns G Ital Ric Appl vol. 3 (2012), p. 384.

[63] Goldberg, L. R. and Rorer, L. G. "Use of two different response modes and repeated testings to predict social conformity." In: Journal of personality and social psychology vol. 3.no. 1 (1966), pp. 2837. DOI: https://doi.org/10.1037/h0022721.

[64] Goldberg, S. C. "Three situational determinants of conformity to social norms." In: The fournal of Abnormal and Social Psychology vol. 49.no. 3 (1954), p. 325. Dor: https://doi.org/10.1037/h0056249.

[65] Gonzalez, L. and Loureiro, Y. K. "When can a photo increase credit? The impact of lender and borrower profiles on online peer-to-peer loans”. In: fournal of Behavioral and Experimental Finance vol. 2 (2014), pp. 44-58. Dor: https://doi.org/10.1016/j.jbef.2014.04.002.

[66] Goodmon, L. B., Gavin, D. J., Urs, M., and Akus, S. N. "The power of the majority: Social conformity in sexual harassment punishment selection”. In: fournal of Applied Social Psychology vol. 50.no. 8 (2020), pp. 441-455. DOI: https://doi.org/10.1111/jasp.12672. 
[67] Griffiths, M. D., Davies, M. N., and Chappell, D. "Breaking the stereotype: The case of online gaming”. In: CyberPsychology \& behavior vol. 6.no. 1 (2003), pp. 81-91. DoI: https://doi.org/10.1089/ 109493103321167992.

[68] Guadagno, R. E. and Cialdini, R. B. "Online persuasion: An examination of gender differences in computer-mediated interpersonal influence." In: Group dynamics: Theory, research, and practice vol. 6.no. 1 (2002), pp. 38-51. DoI: https://doi.org/10.1037/1089-2699.6.1.38.

[69] Guadagno, R. E. and Cialdini, R. B. "Persuade him by email, but see her in person: Online persuasion revisited”. In: Computers in Human Behavior vol. 23.no. 2 (2007), pp. 999-1015. DoI: https://doi.org/ 10.1016/j.chb.2005.08.006.

[70] Gunawardena, C. N. "Social presence theory and implications for interaction and collaborative learning in computer conferences”. In: International journal of educational telecommunications vol. 1.no. 2 (1995), pp. 147-166.

[71] Gunawardena, C. N. and Zittle, F. J. "Social presence as a predictor of satisfaction within a computermediated conferencing environment”. In: American journal of distance education vol. 11.no. 3 (1997), pp. 8-26. DOI: https://doi.org/10.1080/08923649709526970.

[72] Hardy, K. R. “Determinants of conformity and attitude change." In: The fournal of Abnormal and Social Psychology vol. 54.no. 3 (1957), pp. 289-294. DoI: https://doi.org/10.1037/h0048374.

[73] Hassanein, K. and Head, M. "Manipulating perceived social presence through the web interface and its impact on attitude towards online shopping”. In: International journal of human-computer studies vol. 65.no. 8 (2007), pp. 689-708. DoI: https://doi.org/10.1016/j.ijhcs.2006.11.018.

[74] Hassanein, K. S. and Head, M. M. "Building online trust through socially rich web interfaces". In: Proceedings of the 2nd annual conference on privacy, security and trust, Fredericton, New Brunswick, Canada. 2004, pp. 15-22.

[75] Hathaway, S. R. and McKinley, J. C. "Minnesota Multiphasic Personality Inventory; Manual, revised”. In: Psychological Corporation (1951).

[76] Helmreich, R. and Stapp, J. "Short forms of the Texas Social Behavior Inventory (TSBI), an objective measure of self-esteem”. In: Bulletin of the Psychonomic Society vol. 4.no. 5 (1974), pp. 473-475. DOI: https://doi.org/10.3758/BF03334260.

[77] Hertz, N. and Wiese, E. "Influence of agent type and task ambiguity on conformity in social decision making”. In: Proceedings of the Human Factors and Ergonomics Society Annual Meeting vol. 60.no. 1 (2016), pp. 313-317. DoI: https://doi.org/10.1177/1541931213601071.

[78] Hertz, N. and Wiese, E. "Under Pressure: Examining Social Conformity With Computer and Robot Groups”. In: Human Factors vol. 60.no. 8 (2018), pp. 1207-1218. DoI: https:// doi.org/10.1177/ 0018720818788473.

[79] Hettiachchi, D., Sarsenbayeva, Z., Allison, F., Berkel, N. van, Dingler, T., Marini, G., Kostakos, V., and Goncalves, J. "" Hi! I am the Crowd Tasker" Crowdsourcing through Digital Voice Assistants". In: Proceedings of the 2020 CHI Conference on Human Factors in Computing Systems. 2020, pp. 1-14. DOI: https://doi.org/10.1145/3313831.3376320.

[80] Hettiachchi, D., Wijenayake, S., Hosio, S., Kostakos, V., and Goncalves, J. "How Context Influences Cross-Device Task Acceptance in Crowd Work". In: Proceedings of the AAAI Conference on Human Computation and Crowdsourcing. Vol. 8. 1. 2020, pp. 53-62.

[81] Hullman, J., Adar, E., and Shah, P. “The Impact of Social Information on Visual Judgments”. In: Proceedings of the SIGCHI Conference on Human Factors in Computing Systems. CHI '11. Vancouver, BC, Canada: ACM, 2011, pp. 1461-1470. Dor: https://doi.org/10.1145/1978942.1979157.

[82] Insko, C. A., Smith, R. H., Alicke, M. D., Wade, J., and Taylor, S. “Conformity and Group Size: The Concern with Being Right and the Concern with Being Liked”. In: Personality and Social Psychology Bulletin vol. 11.no. 1 (1985), pp. 41-50. Dor: https://doi.org/10.1177/0146167285111004. 
[83] Janis, I. L. and Field, P. B. "Sex differences and personality factors related to persuasibility." In: Personality and persuasibility (1959), pp. 55-68. Dor: https://psycnet.apa.org/record/1960-07155-005.

[84] John, O. P. and Srivastava, S. "The Big Five trait taxonomy: History, measurement, and theoretical perspectives". In: Handbook of personality: Theory and research vol. 2.no. 1999 (1999), pp. 102-138.

[85] Jones, D. “A WEIRD view of human nature skews psychologists' studies". In: Science vol. 328.no. 5986 (2010), p. 1627. Dor: https://doi.org/10.1126/science.328.5986.1627.

[86] Kaye, L. K., Gresty, C. E., and Stubbs-Ennis, N. "Exploring stereotypical perceptions of female players in digital gaming contexts”. In: Cyberpsychology, Behavior, and Social Networking vol. 20.no. 12 (2017), pp. 740-745. DoI: https://doi.org/10.1089/cyber.2017.0294.

[87] Kelman, H. C. "Compliance, identification, and internalization three processes of attitude change". In: Journal of conflict resolution vol. 2.no. 1 (1958), pp. 51-60. DoI: https:// doi.org/10.1177/ 002200275800200106.

[88] Keyes, O. "The misgendering machines: Trans/HCI implications of automatic gender recognition”. In: Proceedings of the ACM on human-computer interaction vol. 2.no. CSCW (2018), pp. 1-22. DoI: https://doi.org/10.1145/3274357.

[89] Kim, J. and Park, H. S. "The effect of uniform virtual appearance on conformity intention: Social identity model of deindividuation effects and optimal distinctiveness theory". In: Computers in Human Behavior vol. 27.no. 3 (2011), pp. 1223-1230. DoI: https://doi.org/10.1016/j.chb.2011.01.002.

[90] Klein, M. H. “Compliance, consistent conformity, and personality.” In: fournal of personality and social psychology vol. 5.no. 2 (1967), pp. 239-245. DoI: https://doi.org/10.1037/h0021203.

[91] Klein, R. L. "Age, sex, and task difficulty as predictors of social conformity". In: Fournal of Gerontology vol. 27.no. 2 (1972), pp. 229-236. DoI: https://doi.org/10.1093/geronj/27.2.229.

[92] Kraemer, P. "Do as we do, not as you think: the effect of group influence on individual choices in a virtual environment”. In: Fournal For Virtual Worlds Research vol. 6.no. 1 (2013), pp. 1-11. DOI: https://doi.org/10.4101/jvwr.v6i1.7002.

[93] Krech, D., Crutchfield, R. S., and Ballachey, E. L. Individual in society: A textbook of social psychology. McGraw-Hill, 1962.

[94] Kumar, J. "Conformity behavior as a function of confederates' age and size of the confederate group.” In: Personality Study \& Group Behaviour vol. 3.no. 2 (1983), pp. 69-73. Dor: https://psycnet. apa.org/record/1984-11929-001.

[95] Kundu, P. and Cummins, D. D. "Morality and conformity: The Asch paradigm applied to moral decisions”. In: Social Influence vol. 8.no. 4 (2013), pp. 268-279. Dor: https://doi.org/10.1080/15534510. 2012.727767.

[96] Kyrlitsias, C. and Michael Grigoriou, D. "Asch conformity experiment using immersive virtual reality”. In: Computer Animation and Virtual Worlds vol. 29.no. 5 (2018), e1804. DoI: https://doi.org/ 10.1002/cav.1804.

[97] Laporte, L., Nimwegen, C. van, and Uyttendaele, A. J. "Do People Say What They Think: Social Conformity Behavior in Varying Degrees of Online Social Presence”. In: Proceedings of the 6th Nordic Conference on Human-Computer Interaction: Extending Boundaries. NordiCHI '10. Reykjavik, Iceland: ACM, 2010, pp. 305-314. DOI: https://doi.org/10.1145/1868914.1868951.

[98] Larsen, K. S. “Conformity in the Asch experiment”. In: The fournal of Social Psychology vol. 94.no. 2 (1974), pp. 303-304. DoI: https://doi.org/10.1080/00224545.1974.9923224.

[99] Larsen, K. S., Triplett, J. S., Brant, W. D., and Langenberg, D. "Collaborator status, subject characteristics, and conformity in the Asch paradigm”. In: The fournal of Social Psychology vol. 108.no. 2 (1979), pp. 259-263. Dor: https://doi.org/10.1080/00224545.1979.9711639.

[100] Lazar, J., Feng, J. H., and Hochheiser, H. Research methods in human-computer interaction. Morgan Kaufmann, 2017. Dor: https://doi.org/10.1016/B978-0-444-70536-5.50047-6. 
[101] Lee, E.-J. "Effects of "gender" of the computer on informational social influence: the moderating role of task type”. In: International fournal of Human-Computer Studies vol. 58.no. 4 (2003), pp. 347-362. DOI: https://doi.org/10.1016/S1071-5819(03)00009-0.

[102] Lee, E.-J. "Effects of gendered character representation on person perception and informational social influence in computer-mediated communication”. In: Computers in Human Behavior vol. 20.no. 6 (2004), pp. 779-799. DoI: https://doi.org/10.1016/j.chb.2003.11.005.

[103] Lee, E.-J. "Effects of visual representation on social influence in computer-mediated communication: Experimental tests of the social identity model of deindividuation effects". In: Human Communication Research vol. 30.no. 2 (2004), pp. 234-259. DoI: https://doi.org/10.1111/j.14682958.2004.tb00732.x.

[104] Lee, E.-J. "When and how does depersonalization increase conformity to group norms in computermediated communication?” In: Communication Research vol. 33.no. 6 (2006), pp. 423-447. DOI: https://doi.org/10.1177/0093650206293248.

[105] Lee, E.-J. "Wired for gender: Experientiality and gender-stereotyping in computer-mediated communication”. In: Media Psychology vol. 10.no. 2 (2007), pp. 182-210. Dor: https://doi.org/ 10.1080/15213260701375595.

[106] Lee, E.-J. and Nass, C. "Experimental tests of normative group influence and representation effects in computer-mediated communication: When interacting via computers differs from interacting with computers". In: Human Communication Research vol. 28.no. 3 (2002), pp. 349-381. DoI: https: //doi.org/10.1111/j.1468-2958.2002.tb00812.x.

[107] Liu, M. and Wang, Y. "Race-of-Interviewer Effect in the Computer-Assisted Self-Interview Module in a Face-to-Face Survey”. In: International Journal of Public Opinion Research vol. 28.no. 2 (2016), pp. 292-305. DOI: https://doi.org/10.1093/ijpor/edv021.

[108] Lucas, G. M., Lehr, J., Krämer, N., and Gratch, J. “The Effectiveness of Social Influence Tactics When Used by a Virtual Agent”. In: Proceedings of the 19th ACM International Conference on Intelligent Virtual Agents. IVA '19. Paris, France: Association for Computing Machinery, 2019, pp. 22-29. DOI: https://doi.org/10.1145/3308532.3329464.

[109] Mandler, G. and Sarason, S. B. “A study of anxiety and learning." In: The fournal of Abnormal and Social Psychology vol. 47.no. 2 (1952), pp. 166-173. DoI: https://doi.org/10.1037/h0062855.

[110] Maruyama, M., Robertson, S. P., Douglas, S., Raine, R., and Semaan, B. "Social Watching a Civic Broadcast: Understanding the Effects of Positive Feedback and Other Users' Opinions”. In: Proceedings of the 2017 ACM Conference on Computer Supported Cooperative Work and Social Computing. CSCW '17. Portland, Oregon, USA: ACM, 2017, pp. 794-807. DoI: https://doi.org/10. 1145/2998181.2998340.

[111] Maruyama, M. T., Robertson, S. P., Douglas, S. K., Semaan, B. C., and Faucett, H. A. "Hybrid Media Consumption: How Tweeting During a Televised Political Debate Influences the Vote Decision”. In: Proceedings of the 17th ACM Conference on Computer Supported Cooperative Work \& Social Computing. CSCW '14. Baltimore, Maryland, USA: ACM, 2014, pp. 1422-1432. Dor: https: //doi.org/10.1145/2531602.2531719.

[112] Maslach, C., Santee, R. T., and Wade, C. "Individuation, gender role, and dissent: Personality mediators of situational forces." In: Journal of personality and social psychology vol. 53.no. 6 (1987), pp. 1088-1093. Dor: https://doi.org/10.1037/0022-3514.53.6.1088.

[113] Maslach, C., Stapp, J., and Santee, R. T. "Individuation: Conceptual analysis and assessment." In: Journal of Personality and Social Psychology vol. 49.no. 3 (1985), pp. 729-738. Dor: https: //doi.org/10.1037/0022-3514.49.3.729.

[114] McDavid Jr, J. "Personality and situational determinants of conformity." In: The fournal of Abnormal and Social Psychology vol. 58.no. 2 (1959), pp. 241-246. Dor: https://doi.org/10.1037/h0039837. 
[115] McGhee, P. E. and Teevan, R. C. "Conformity behavior and need for affiliation”. In: The fournal of social psychology vol. 72.no. 1 (1967), pp. 117-121. DoI: https://doi.org/10.1080/00224545.1967. 9922305.

[116] McHugh, M. L. "The chi-square test of independence”. In: Biochemia medica vol. 23.no. 2 (2013), pp. 143-149. DoI: https://doi.org/10.11613/BM.2013.018.

[117] McKelvey, W. and Kerr, N. H. "Differences in conformity among friends and strangers". In: Psychological Reports vol. 62.no. 3 (1988), pp. 759-762. DoI: https://doi.org/10.2466/pr0.1988.62.3.759.

[118] McKenna, K. Y. and Green, A. S. "Virtual group dynamics.” In: Group Dynamics: Theory, Research, and Practice vol. 6.no. 1 (2002), pp. 116-127. Dor: https://doi.org/10.1037/1089-2699.6.1.116.

[119] Meunier, C. and Rule, B. G. “Anxiety, confidence, and conformity 1”. In: fournal of Personality vol. 35.no. 3 (1967), pp. 498-504. Dor: https://doi.org/10.1111/j.1467-6494.1967.tb01443.x.

[120] Midden, C., Ham, J., and Baten, J. "Conforming to an artificial majority: persuasive effects of a group of artificial agents”. In: International Conference on Persuasive Technology. Springer, 2015, pp. 229-240. Dor: https://doi.org/10.1007/978-3-319-20306-5_21.

[121] Mori, K. and Arai, M. "No need to fake it: Reproduction of the Asch experiment without confederates”. In: International fournal of Psychology vol. 45.no. 5 (2010), pp. 390-397. DoI: https: //doi.org/10.1080/00207591003774485.

[122] Morris, W. N. and Miller, R. S. "The effects of consensus-breaking and consensus-preempting partners on reduction of conformity”. In: fournal of Experimental Social Psychology vol. 11.no. 3 (1975), pp. 215-223. DOI: https://doi.org/10.1016/S0022-1031(75)80023-0.

[123] Mouton, J. S., Blake, R. R., and Olmstead, J. A. "The Relationship between Frequency of Yielding and the Disclosure of Personal Identity1". In: Journal of Personality vol. 24.no. 3 (1956), pp. 339-347. DOI: https://doi.org/10.1111/j.1467-6494.1956.tb01273.x.

[124] Mugny, G. "Compliance, conversion and the Asch paradigm”. In: European fournal of Social Psychology vol. 14.no. 4 (1984), pp. 353-368. DoI: https://doi.org/10.1002/ejsp.2420140402.

[125] Nemeth, C. and Chiles, C. "Modelling courage: The role of dissent in fostering independence". In: European fournal of Social Psychology vol. 18.no. 3 (1988), pp. 275-280. DOI: https://doi.org/10.1002/ ejsp.2420180306.

[126] Nicholson, N., Cole, S. G., and Rocklin, T. "Conformity in the Asch situation: A comparison between contemporary British and US university students”. In: British fournal of Social Psychology vol. 24.no. 1 (1985), pp. 59-63. Dor: https://doi.org/10.1111/j.2044-8309.1985.tb00660.x.

[127] Olsson, T., Lagerstam, E., Kärkkäinen, T., and Väänänen-Vainio-Mattila, K. "Expected user experience of mobile augmented reality services: a user study in the context of shopping centres". In: Personal and ubiquitous computing vol. 17.no. 2 (2013), pp. 287-304. DoI: https://doi.org/10.1007/ s00779-011-0494-X.

[128] Olsson, T. and Salo, M. “Online user survey on current mobile augmented reality applications”. In: 2011 10th IEEE International Symposium on Mixed and Augmented Reality. IEEE. 2011, pp. 75-84. DOI: https://doi.org/10.1109/ISMAR.2011.6092372.

[129] Ozok, A. A. "Survey design and implementation in HCI". In: The human-computer interaction handbook. CRC Press, 2007, pp. 1177-1196.

[130] Packer, D. J. "The interactive influence of conscientiousness and openness to experience on dissent". In: Social Influence vol. 5.no. 3 (2010), pp. 202-219. DoI: https://doi.org/10.1080/15534511003676441.

[131] Pak, R., McLaughlin, A. C., and Bass, B. "A multi-level analysis of the effects of age and gender stereotypes on trust in anthropomorphic technology by younger and older adults”. In: Ergonomics vol. 57.no. 9 (2014), pp. 1277-1289. DOI: https://doi.org/10.1080/00140139.2014.928750.

[132] Pasupathi, M. "Age differences in response to conformity pressure for emotional and nonemotional material.” In: Psychology and aging vol. 14.no. 1 (1999), pp. 170-174. Dor: https://doi.org/10.1037/ 0882-7974.14.1.170. 
[133] Payne, G. and Payne, J. “The Hawthorne Effect”. In: Key Concepts in Social Research (2004), pp. 108111. DoI: https://dx.doi.org/10.4135/9781849209397.

[134] Perfumi, S. C., Bagnoli, F., Caudek, C., and Guazzini, A. "Deindividuation effects on normative and informational social influence within computer-mediated-communication”. In: Computers in Human Behavior vol. 92 (2019), pp. 230-237. DoI: https://doi.org/10.1016/j.chb.2018.11.017.

[135] Perrin, S. and Spencer, C. "Independence or conformity in the Asch experiment as a reflection of cultural and situational factors”. In: British fournal of Social Psychology vol. 20.no. 3 (1981), pp. 205-209. DOI: https://doi.org/10.1111/j.2044-8309.1981.tb00533.x.

[136] Postmes, T., Spears, R., Sakhel, K., and De Groot, D. "Social influence in computer-mediated communication: The effects of anonymity on group behavior”. In: Personality and Social Psychology Bulletin vol. 27.no. 10 (2001), pp. 1243-1254. Dor: https://doi.org/10.1177/01461672012710001.

[137] Reysen, M. “The effects of conformity on recognition judgements”. In: Memory vol. 13.no. 1 (2005), pp. 87-94. DOI: https://doi.org/10.1080/09658210344000602.

[138] Roccas, S., Sagiv, L., Schwartz, S. H., and Knafo, A. "The big five personality factors and personal values”. In: Personality and social psychology bulletin vol. 28.no. 6 (2002), pp. 789-801. DoI: https: //doi.org/10.1177/0146167202289008.

[139] Rosander, M. and Eriksson, O. "Conformity on the Internet-The role of task difficulty and gender differences”. In: Computers in human behavior vol. 28.no. 5 (2012), pp. 1587-1595. DoI: https: //doi.org/10.1016/j.chb.2012.03.023.

[140] Rose, J., Mackey-Kallis, S., Shyles, L., Barry, K., Biagini, D., Hart, C., and Jack, L. "Face it: The impact of gender on social media images”. In: Communication Quarterly vol. 60.no. 5 (2012), pp. 588-607. DOI: https://doi.org/10.1080/01463373.2012.725005.

[141] Rosenberg, L. “Group size, prior experience, and conformity.” In: The fournal of Abnormal and Social Psychology vol. 63.no. 2 (1961), pp. 436-437. DOI: https://doi.org/10.1037/h0047007.

[142] Rosenberg, L. A. "Conformity as a function of confidence in self and confidence in partner". In: Human Relations vol. 16.no. 2 (1963), pp. 131-139. DoI: https://doi.org/10.1177/001872676301600202.

[143] Rosenberg, M. "Rosenberg self-esteem scale (RSE)". In: Acceptance and commitment therapy. Measures package vol. 61.no. 52 (1965), p. 18.

[144] Ross, L., Bierbrauer, G., and Hoffman, S. "The role of attribution processes in conformity and dissent: Revisiting the Asch situation.” In: American Psychologist vol. 31.no. 2 (1976), p. 148. DOI: https://doi.org/10.1037/0003-066X.31.2.148.

[145] Rugs, D. and Kaplan, M. F. "Effectiveness of informational and normative influences in group decision making depends on the group interactive goal”. In: British fournal of Social Psychology vol. 32.no. 2 (1993), pp. 147-158. Dor: https://doi.org/10.1111/j.2044-8309.1993.tb00992.x.

[146] Salomons, N., Linden, M. van der, Strohkorb Sebo, S., and Scassellati, B. "Humans Conform to Robots: Disambiguating Trust, Truth, and Conformity". In: Proceedings of the 2018 ACM/IEEE International Conference on Human-Robot Interaction. HRI '18. Chicago, IL, USA: Association for Computing Machinery, 2018, pp. 187-195. DoI: https://doi.org/10.1145/3171221.3171282.

[147] Salomons, N., Sebo, S. S., Qin, M., and Scassellati, B. "A Minority of One against a Majority of Robots: Robots Cause Normative and Informational Conformity”. In: ACM Transactions on Human-Robot Interaction (THRI) vol. 10.no. 2 (2021), pp. 1-22. Dor: https://doi.org/10.1145/3442627.

[148] Santee, R. T. and Maslach, C. "To agree or not to agree: Personal dissent amid social pressure to conform.” In: Journal of Personality and Social Psychology vol. 42.no. 4 (1982), pp. 690-700. DoI: https://doi.org/10.1037/0022-3514.42.4.690.

[149] Sarsenbayeva, Z., Berkel, N. van, Jiang, W., Hettiachchi, D., Kostakos, V., and Goncalves, J. "Effect of Ambient Light on Mobile Interaction”. In: IFIP Conference on Human-Computer Interaction. Springer. 2019, pp. 465-475. DoI: https://doi.org/10.1007/978-3-030-29387-1_26. 
[150] Sassenberg, K. and Postmes, T. "Cognitive and strategic processes in small groups: Effects of anonymity of the self and anonymity of the group on social influence”. In: British fournal of Social Psychology vol. 41.no. 3 (2002), pp. 463-480. Dor: https://doi.org/10.1348/014466602760344313.

[151] Schneider, D. M. and Watkins, M. J. "Response conformity in recognition testing”. In: Psychonomic bulletin \& review vol. 3.no. 4 (1996), pp. 481-485. DoI: https://doi.org/10.3758/BF03214550.

[152] Schneider, F. "Recommended by Google Home”. In: International Conference on Human-Computer Interaction. Springer, Cham. 2021, pp. 485-493. DoI: https://doi.org/10.1007/978-3-030-78635-9_63.

[153] Schulman, G. I. “Asch Conformity Studies: Conformity to the Experimenter and/or to the Group?" In: Sociometry (1967), pp. 26-40. DOI: https://doi.org/10.2307/2786436.

[154] Sharma, E. and De Choudhury, M. "Mental Health Support and Its Relationship to Linguistic Accommodation in Online Communities". In: Proceedings of the 2018 CHI Conference on Human Factors in Computing Systems. CHI '18. Montreal QC, Canada: ACM, 2018, 641:1-641:13. DOI: https://doi.org/10.1145/3173574.3174215.

[155] Shiomi, M. and Hagita, N. “Do Synchronized Multiple Robots Exert Peer Pressure?” In: Proceedings of the Fourth International Conference on Human Agent Interaction. HAI '16. Biopolis, Singapore: Association for Computing Machinery, 2016, pp. 27-33. Dor: https://doi.org/10.1145/2974804. 2974808.

[156] Short, J., Williams, E., and Christie, B. The social psychology of telecommunications. John Wiley \& Sons, 1976.

[157] Sistrunk, F. and McDavid, J. W. "Achievement motivation, affiliation motivation, and task difficulty as determinants of social conformity”. In: The fournal of social psychology vol. 66.no. 1 (1965), pp. 41-50. DOI: https://doi.org/10.1080/00224545.1965.9919619.

[158] Sistrunk, F. "Two processes of conformity demonstrated by interactions of commitment, set, and personality”. In: The fournal of social psychology vol. 89.no. 1 (1973), pp. 63-72. Dor: https: //doi.org/10.1080/00224545.1973.9922568.

[159] Smilowitz, M., Compton, D. C., and Flint, L. "The effects of computer mediated communication on an individual's judgment: A study based on the methods of Asch's social influence experiment". In: Computers in Human Behavior vol. 4.no. 4 (1988), pp. 311-321. DoI: https://doi.org/10.1016/07475632(88)90003-9.

[160] Smith, K. H. "Ego strength and perceived competence as conformity variables." In: The fournal of Abnormal and Social Psychology vol. 62.no. 1 (1961), pp. 169-171. DoI: https://doi.org/10.1037/ h0041051.

[161] Snyder, C. R. and Fromkin, H. L. "Abnormality as a positive characteristic: The development and validation of a scale measuring need for uniqueness.” In: fournal of Abnormal Psychology vol. 86.no. 5 (1977), pp. 518-527. DoI: https://doi.org/10.1037/0021-843X.86.5.518.

[162] Spears, R., Lea, M., and Lee, S. "De-individuation and group polarization in computer-mediated communication”. In: British fournal of Social Psychology vol. 29.no. 2 (1990), pp. 121-134. DOI: https://doi.org/10.1111/j.2044-8309.1990.tb00893.x.

[163] Spielberger, C. D. "State-trait anxiety inventory for adults". In: APA PsycTests (1983). DoI: https: //doi.org/10.1037/t06496-000.

[164] Stang, D. J. “Group size effects on conformity”. In: The fournal of Social Psychology vol. 98.no. 2 (1976), pp. 175-181. DoI: https://doi.org/10.1080/00224545.1976.9923388.

[165] Stang, D. J. "Ineffective deception in conformity research: Some causes and consequences". In: European fournal of Social Psychology vol. 6.no. 3 (1976), pp. 353-367. DoI: https://doi.org/10.1002/ ejsp.2420060306.

[166] Steiner, I. D. and Vannoy, J. S. "Personality correlates of two types of conformity behavior". In: Journal of Personality and Social Psychology vol. 4.no. 3 (1966), pp. 307-315. DoI: https://doi.org/10. 1037/h0023707. 
[167] Strickland, B. R. and Crowne, D. P. "Conformity under conditions of simulated group pressure as a function of the need for social approval”. In: The fournal of Social Psychology vol. 58.no. 1 (1962), pp. 171-181. DOI: https://doi.org/10.1080/00224545.1962.9712366.

[168] Sukumaran, A., Vezich, S., McHugh, M., and Nass, C. "Normative Influences on Thoughtful Online Participation". In: Proceedings of the SIGCHI Conference on Human Factors in Computing Systems. CHI '11. Vancouver, BC, Canada: ACM, 2011, pp. 3401-3410. DoI: https://doi.org/10.1145/1978942. 1979450.

[169] Tesser, A., Campbell, J., and Mickler, S. "The role of social pressure, attention to the stimulus, and self-doubt in conformity”. In: European fournal of Social Psychology vol. 13.no. 3 (1983), pp. 217-233. DOI: https://doi.org/10.1002/ejsp.2420130303.

[170] Tu, C.-H. and McIsaac, M. "The relationship of social presence and interaction in online classes". In: The American journal of distance education vol. 16.no. 3 (2002), pp. 131-150. Dor: https://doi.org/ 10.1207/S15389286AJDE1603_2.

[171] Van Berkel, N., Goncalves, J., Hettiachchi, D., Wijenayake, S., Kelly, R. M., and Kostakos, V. "Crowdsourcing perceptions of fair predictors for machine learning: a recidivism case study". In: Proceedings of the ACM on Human-Computer Interaction vol. 3.no. CSCW (2019), pp. 1-21. DOI: https://doi.org/10.1145/3359130.

[172] Vollmer, A.-L., Read, R., Trippas, D., and Belpaeme, T. “Children conform, adults resist: A robot group induced peer pressure on normative social conformity”. In: Science Robotics vol. 3.no. 21 (2018). DOI: https://doi.org/10.1126/scirobotics.aat7111.

[173] Vosoughi, S., Roy, D., and Aral, S. "The spread of true and false news online". In: Science vol. 359.no. 6380 (2018), pp. 1146-1151. Dor: https://doi.org/10.1126/science.aap9559.

[174] Walker, L. S. "Developing Your Experiment”. In: Laboratory Experiments in the Social Sciences (2nd Edition). Academic Press, 2014, pp. 127-144. DoI: https://doi.org/10.1016/B978-0-12-4046818.00006-6.

[175] Walker, M. B. and Andrade, M. G. "Conformity in the Asch task as a function of age". In: The Journal of social psychology vol. 136.no. 3 (1996), pp. 367-372. DoI: https://doi.org/10.1080/00224545. 1996.9714014.

[176] Walther, E., Bless, H., Strack, F., Rackstraw, P., Wagner, D., and Werth, L. "Conformity effects in memory as a function of group size, dissenters and uncertainty”. In: Applied Cognitive Psychology: The Official fournal of the Society for Applied Research in Memory and Cognition vol. 16.no. 7 (2002), pp. 793-810. DOI: https://doi.org/10.1002/acp.828.

[177] Widiger, T. A. and Oltmanns, J. R. "Neuroticism is a fundamental domain of personality with enormous public health implications”. In: World Psychiatry vol. 16.no. 2 (2017), pp. 144-145. DOI: https://doi.org/10.1002/wps.20411.

[178] Wijenayake, S., Berkel, N. van, and Goncalves, J. "Bots for research: Minimising the experimenter effect”. In: International Workshop on Detection and Design for Cognitive Biases in People and Computing Systems (CHI'20 Workshop). ACM. 2020, pp. 1-9.

[179] Wijenayake, S., Berkel, N. van, Kostakos, V., and Goncalves, J. "Impact of contextual and personal determinants on online social conformity”. In: Computers in Human Behavior vol. 108.no. 106302 (2020), pp. 1-11. DoI: https://doi.org/10.1016/j.chb.2020.106302.

[180] Wijenayake, S., Berkel, N. van, Kostakos, V., and Goncalves, J. "Measuring the Effects of Gender on Online Social Conformity”. In: Proc. ACM Hum.-Comput. Interact. vol. 3.no. CSCW (2019), 145:1-145:24. DOI: https://doi.org/10.1145/3359247.

[181] Wijenayake, S., Berkel, N. van, Kostakos, V., and Goncalves, J. "Quantifying the Effect of Social Presence on Online Social Conformity”. In: Proceedings of the ACM on Human-Computer Interaction vol. 4.no. CSCW1 (2020), pp. 1-22. Dor: https://doi.org/10.1145/3392863. 
[182] Wijenayake, S., Hettiachchi, D., Hosio, S., Kostakos, V., and Goncalves, J. "Effect of Conformity on Perceived Trustworthiness of News in Social Media”. In: IEEE Internet Computing vol. 25.no. 1 (2021), pp. 12-19. DOI: https://doi.org/10.1109/MIC.2020.3032410.

[183] Wijenayake, S., Hu, J., Kostakos, V., and Goncalves, J. "Quantifying the Effects of Age-Related Stereotypes on Online Social Conformity”. In: IFIP Conference on Human-Computer Interaction. Springer. 2021, pp. 451-475. DOI: https://doi.org/10.1007/978-3-030-85610-6_26.

[184] Wilson, S. R., Brown, N. L., Mejia, C., and Lavori, P. W. "Effects of interviewer characteristics on reported sexual behavior of California Latino couples". In: Hispanic fournal of Behavioral Sciences vol. 24.no. 1 (2002), pp. 38-62. DoI: https://doi.org/10.1177/0739986302024001003.

[185] Wood, W. and Karten, S. J. "Sex differences in interaction style as a product of perceived sex differences in competence.” In: fournal of personality and social psychology vol. 50.no. 2 (1986), pp. 341-347. DOI: https://doi.org/10.1037/0022-3514.50.2.341.

[186] Xu, K. and Lombard, M. "Persuasive computing: Feeling peer pressure from multiple computer agents”. In: Computers in Human Behavior vol. 74 (2017), pp. 152-162. DoI: https://doi.org/10.1016/ j.chb.2017.04.043.

[187] Yarnold, P. R., Grimm, L. G., and Mueser, K. T. "Social conformity and the Type A behavior pattern". In: Perceptual and motor skills vol. 62.no. 1 (1986), pp. 99-104. DoI: https://doi.org/10.2466/pms. 1986.62.1.99.

[188] Zhu, H., Huberman, B., and Luon, Y. “To Switch or Not to Switch: Understanding Social Influence in Online Choices”. In: Proceedings of the SIGCHI Conference on Human Factors in Computing Systems. CHI '12. Austin, Texas, USA: ACM, 2012, pp. 2257-2266. DoI: https://doi.org/10.1145/2207676. 2208383.

[189] Zonca, J., Folso, A., and Sciutti, A. "Dynamic modulation of social influence by indirect reciprocity". In: Scientific reports vol. 11.no. 1 (2021), pp. 1-14. Dor: https://doi.org/10.1038/s41598-021-90656-y. 


\section{University Library}

\section{- M I I N E R VA \\ A gateway to Melbourne's research publications}

Minerva Access is the Institutional Repository of The University of Melbourne

Author/s:

Wijenayake, Senuri Samalka

Title:

Understanding the Dynamics of Online Social Conformity

Date:

2021

Persistent Link:

http://hdl.handle.net/11343/295771

Terms and Conditions:

Terms and Conditions: Copyright in works deposited in Minerva Access is retained by the copyright owner. The work may not be altered without permission from the copyright owner. Readers may only download, print and save electronic copies of whole works for their own personal non-commercial use. Any use that exceeds these limits requires permission from the copyright owner. Attribution is essential when quoting or paraphrasing from these works. 